


\title{
QUANTUM INFORMATION PROCESSING IN LARGE-SCALE PHOTONIC NETWORKS
}

\author{
Quantuminformatieverwerking \\ in grootschalige fotonische netwerken
}


Dit proefschrift is goedgekeurd door:

Promotor:

prof. dr. P.W.H. Pinkse

Co-promotor:

dr. J.J. Renema

Cover: $\quad$ Concept and design by M. Adhikary and R. van der Meer

Printed by Gildeprint - Enschede

ISBN: $\quad$ 978-90-365-5334-6

DOI: $\quad 10.3990 / 1.9789036553346$

Various setup figures are based on the ComponentLibrary by Alexander Franzen, used under CC BY-NC 3.0.

(C)2022, R. van der Meer, Enschede, The Netherlands. All rights reserved. No parts of this thesis may be reproduced, stored in a retrieval system or transmitted in any form or by any means without permission of the author.

Alle rechten voorbehouden. Niets uit deze uitgave mag worden vermenigvuldigd, in enige vorm of op enige wijze, zonder voorafgaande schriftelijke toestemming van de auteur. 


\section{QUANTUM INFORMATION PROCESSING IN LARGE-SCALE PHOTONIC NETWORKS}

\section{PROEFSCHRIFT}

ter verkrijging van

de graad van doctor aan de Universiteit Twente,

op gezag van de rector magnificus,

prof. dr. ir. A. Veldkamp

volgens besluit van het College voor Promoties

in het openbaar te verdedigen

op donderdag 24 februari 2022 om 12.45 uur

door

Reinier van der Meer

geboren op 7 februari 1993

te Gouda, Nederland 


\section{Promotie commissie}

Voorzitter en secretaris

Promotor

Co-promotor

Overige leden prof. dr. J.L. Herek

prof. dr. P.W.H. Pinkse

dr. J.J. Renema

prof. dr. ir. D.A.I. Marpaung

prof. dr. ir. W.G. van der Wiel

prof. dr. ir. H.J. Broersma

prof. dr. E. Diamanti

prof. dr. F. Sciarrino

prof. dr. ir. Hanson

The work described in this thesis was carried out in the

Adaptive Quantum Optics group,

MESA+ Institute for Nanotechnology,

Faculty of Science and Technology,

University of Twente, P.O. Box 217,

7500 AE Enschede, The Netherlands.

This work was financially supported by the Nederlandse Organisatie voor Wetenschappelijk Onderzoek (NWO) via the QuantERA QUOMPLEX project (Grant No. 680.91.037) and via the "SMOKE" project (Grant No. CS.008). 


\section{Contents}

1 Introduction 1

1.1 The required hardware ................... 4

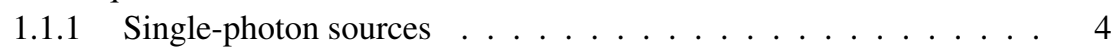

1.1.2 Large optical networks . . . . . . . . . . . . . . 5

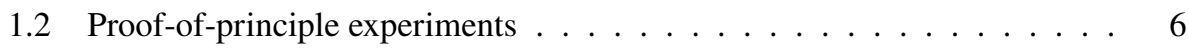

1.2.1 High fidelity control over the network . . . . . . . . . . . 7

1.2.2 Certification of entanglement . . . . . . . . . . . . 7

1.2.3 Thermalization of pure quantum states . . . . . . . . . . 8

1.3 Thesis outline .................... 8

2 Boson sampling in low-depth optical systems 11

2.1 Thin scattering systems . . . . . . . . . . . . . . . . . . . . 13

2.2 Boson Sampling . . . . . . . . . . . . . . . . . . . . . . . . . . . . . . 14

2.3 Overview of results . . . . . . . . . . . . . . . . 16

2.4 Truncations approach i.i.d. Gaussian . . . . . . . . . . . . . . . 17

2.4.1 Analytical results . . . . . . . . . . . . . . . . 18

2.4.2 Eigenvalue spectra . . . . . . . . . . . . . . . . . 19

2.4 .3 Permanents ........................ 21

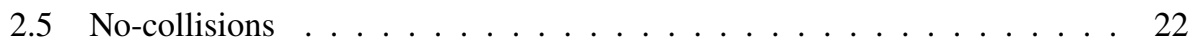

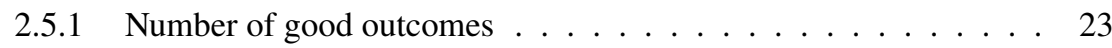

2.5 .2 Numerical results . . . . . . . . . . . . . . . . . . 24

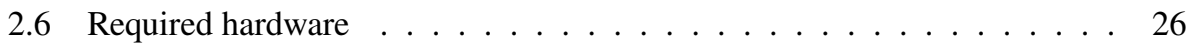

2.7 Conclusion . . . . . . . . . . . . . . . . . 27

3 Spontaneous parametric downconversion for boson sampling 29

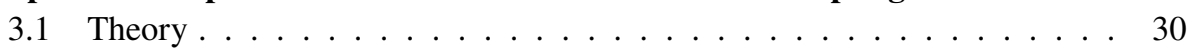

3.1.1 SPDC sources . . . . . . . . . . . . . 30

3.1.2 Classical simulation of boson sampling with imperfections . . . . 33

3.2 Methods . . . . . . . . . . . . . . . . . . . . . . 34

3.3 Results . . . . . . . . . . . . . . . . . . . . . . . . . . . . . . .

3.4 Discussion . . . . . . . . . . . . . . . . . 37

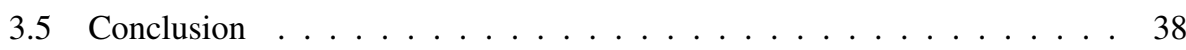

Appendices

3.A Effect of multiphoton states on the purity . . . . . . . . . . . . 39

3.B Optimal SPDC settings . . . . . . . . . . . . . . . . . . . . . . . . . . 40

3.C Numerical stability . . . . . . . . . . . . . . . . . . . 40

4 Multiphoton setup $\quad 45$

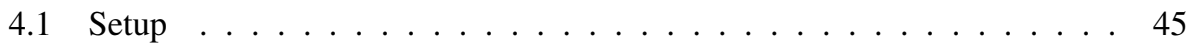

4.1.1 Multiphoton source . . . . . . . . . . . . . 45

4.2 Generation rates . . . . . . . . . . . . . . . . . 48 
4.3 Spectral properties . . . . . . . . . . . . . . . . . . . . . 49

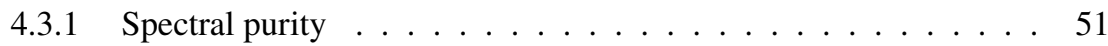

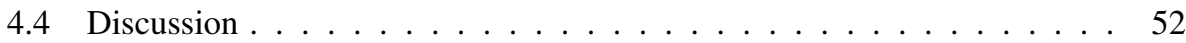

Appendices

4.A Detector settings ........................ 54

5 Integrated quantum processor $\mathbf{5 5}$

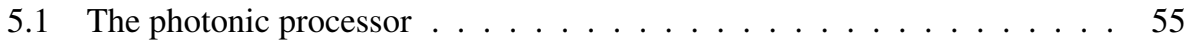

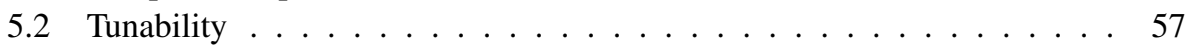

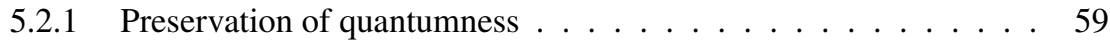

5.2 .2 Thermal photon source . . . . . . . . . . . . . 59

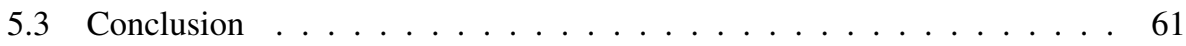

6 Observation of open scattering channels 63

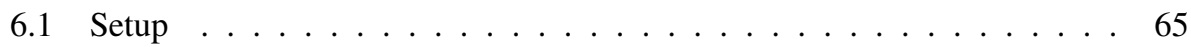

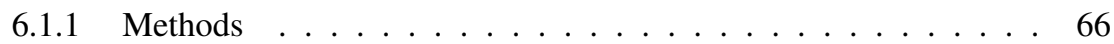

6.2 Open Channels. . . . . . . . . . . . . . . . . . . . . . 67

6.3 Mode filtering. . . . . . . . . . . . . . . . . . . 70

6.4 Discussion . . . . . . . . . . . . . . . . . 70

Appendices

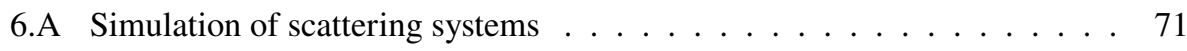

6.B Matrix embedding . . . . . . . . . . . . . . . 71

7 An efficient, one-sided device-independent photonic indistinguishability witness $\quad \mathbf{7 5}$

7.1 Indistinguishability witness . . . . . . . . . . . . . 76

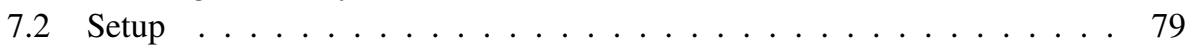

7.3 Results . . . . . . . . . . . . . . . . . 80

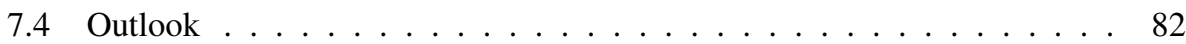

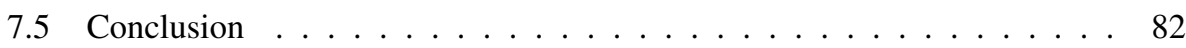

Appendices

7.A Noisy ideal matrix . . . . . . . . . . . . . . . . . . . 83

7.B Monte-Carlo search for the ideal matrix . . . . . . . . . . . . . 83

8 Quantum photo-thermodynamics on a programmable photonic quantum pro$\begin{array}{lr}\text { cessor } & 87\end{array}$

8.1 Local equilibration and thermalization . . . . . . . . . . . . . . 88

8.2 Certification . . . . . . . . . . . . . . . . . . . . . . . . . . 90

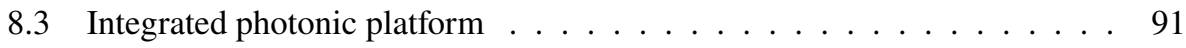

8.4 Results . . . . . . . . . . . . . . . . . . . . . 94

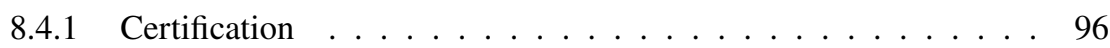




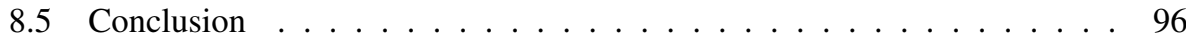

Appendices

8.A Derivation of fidelity witness . . . . . . . . . . . . . . 98

8.B Local Photon Statistics in Finite-size systems . . . . . . . . . . . . . . 102

8.C Experimental methods . . . . . . . . . . . . . . . . 106

8.D Statistics of certification . . . . . . . . . . . . 110

9 Summary and outlook 115

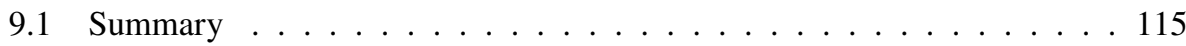

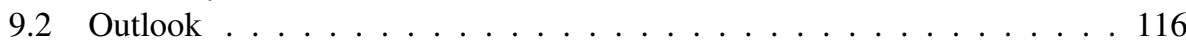

9.2.1 Improving the multiphoton source . . . . . . . . . . . 117

9.2.2 Future integrated optical networks . . . . . . . . . . . . 118

$\begin{array}{ll}\text { Bibliography } & 121\end{array}$

$\begin{array}{ll}\text { Nederlandse samenvatting } & 141\end{array}$

$\begin{array}{ll}\text { Acknowledgements } & 143\end{array}$

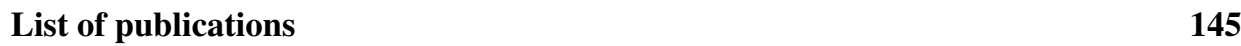





\section{Introduction}

Quantum mechanics has both puzzled and fascinated people with counter-intuitive phenomena. Examples are quantum superposition, entanglement and teleportation [1]. Superposition is the situation where a single particle can be in multiple states, e.g. positions, at the same time. Entanglement induces correlations between quantum particles that are stronger than classically allowed. Finally, quantum teleportation allows to transfer properties of a quantum state from one particle to another particle, without physically moving them. Quantum physics does not only intrigue the general public: scientists too are struggling with the description of physical systems with quantum mechanics. A notable example of a scientific struggle is to fully describe a large and complicated quantum system, such as a molecule, in full detail. Loosely speaking, anything more complicated than solving a particle in a box or a hydrogen atom already becomes challenging to do analytically with pen and paper.

The idea that small quantum systems can be simulated on computers, but that larger systems quickly become intractable for computers, led Yuri Manin in 1980, and shortly after Richard Feynman in 1982, to conjecture that a well-controlled quantum system can be used to simulate other quantum systems [2,3]. This realization is considered to be the origin of the concept of quantum simulators and quantum computers. It inspired the development of the first quantum algorithms in the 1990's such as prime factoring [4] and unstructured search [5]. Furthermore, the first quantum error-correction schemes were proposed to correct for noise on the fragile quantum states [6].

Nowadays, the field has evolved substantially and quantum simulators and quantum computers are expected to contribute to many different fields. Typical examples for applications of quantum systems are biochemistry [7], quantum machine learning [8], and a quantum internet to connect to and communicate between quantum computers [9]. With ever increasing expectations of quantum simulators and computers, the focus is now on developing devices that can actually implement all these ideas. However, the feasibility of full-fledged quantum simulators and computers is still under debate.

Even if a fault-tolerant quantum computer will turn out to be feasible, they will require millions of qubits and improved control over these qubits to meet the high expectations. The current state of the art is far more sobering than the current expectations: only systems with about $50-100$ qubits are reported [10-12]. This is still far away from millions of qubits required for fault-tolerant computing. The current small quantum devices are typically referred to as noisy, intermediate-scale quantum (NISQ) devices. The noise in such devices disturbs the fragile quantum states and the number of qubits is still too small to implement any quantum error correction scheme.

It was expected that NISQ devices would help the development of future quantum systems in a few ways. Firstly, it encourages the development of better quantum systems by showing quantum simulators and computers can actually exist. Secondly, it was expected that NISQ systems will enable quantum simulations that would give some kind of quantum advantage, or quantum speedup compared to classical (super)computers. Unfortunately, at the moment this seems unlikely as most of the current technology is still too noisy [13]. This does not mean that NISQ devices are not interesting and entirely useless! They 
encourage to study the limits between quantum and classical simmulability. This has so far been an interesting and rewarding endeavor, both for obtaining new theoretical insights and as a guide to set up new experiments.

An interesting and fundamental problem that can be tackled with a NISQ system is investigating the so-called Shor's trilemma. This trilemma is a contradiction at a fundamental level among three well-understood and established fields of science and important axioms in these fields. These fields are: computer science, complexity theory and quantum physics.

The first field of Shor's trilemma is computer science. One of the central concepts in computer science is the Turing machine, a mathematical model of computer that is considered to apply to all types of computation. This axiom is formulated by the 'Extended Church-Turing thesis', which states that "a probabilistic Turing machine can efficiently simulate any realistic model of computation" [14]. In other words: all computational problems that are efficiently solvable by realistic physical devices, are solvable by a probabilistic Turing machine [15]. Consequently, computer science insists that all types of computers are the same at a fundamental level. Hardware specifications can drastically vary from one computer to the next, but all types of computers are bound to a similar scaling for a specific task, meaning there can only be a polynomial speed-up in alternate computer architectures.

The second field is complexity theory, a branch of mathematics. Complexity theorists have good reason to believe that the complexity classes $\mathrm{P} \neq \mathrm{NP}$, meaning that there are certain computational tasks that are easy to verify, but not necessarily easy to solve. An example of such a computational task is the famous problem of factoring a large integer into its two prime factors [4]. This mathematical problem is strongly believed to be either in NP or even NP-intermediate and is at the foundation of public-key cryptography protocols such as RSA.

The third field involved in Shor's trilemma is quantum physics. Quantum physics predicts the existence of quantum computers that can solve some specific computational problems much faster than their classical counterparts. Perhaps the most famous example of all quantum algorithms is Shor's algorithm [4]. Shor's algorithm allows to efficiently factor a large integer into primes. Efficient means the algorithms run time scales polynomially when the problem size is increased.

Now the trilemma becomes apparent: if a quantum computer can indeed execute Shor's algorithm efficiently, then it must be a realistic physical computational device. Accordingly, the extended Church-Turning thesis now implies that there must be a classical algorithm that can do the prime factorization efficiently; after all all realistic forms of computers are essentially the same. However, this would imply that computational complexity is not well understood as it claims that prime factorization is a computationally hard problem that cannot be solved efficiently. Alternatively, it is possible that there is no classical computer that can efficiently factorize primes, which would disprove the extended Church-Turning thesis. Finally, it could be that quantum computers are simply not possible, maybe because of some still unknown fundamental limitation to the amount of noise that disturbs the fragile quantum states. Regardless of which concept turns out to 
be wrong, at least one of the fields misunderstands some central concept of their field.

Finding out which of these three axioms has to yield is currently an active field of research by various quantum platforms. The strategy in these experiments is to find some computational task, no matter how useless or obscure, of which the computational complexity is well understood and that is known to have an exponential scaling in run time. Aaronson and Arkhipov introduced in 2011 the idea of boson sampling [15] to do exactly that. Soon afterward, a similar experiment was proposed for superconducting qubits: random circuit sampling [16]. Both proposals require about 50 not too noisy quantum particles to interact randomly and then sample from the resulting quantum state. It is shown that calculating the probability of even a single possible outcome is classically infeasible, whereas a (noisy) quantum system can sample from the distribution on the fly. Note that these experiments do not simulate some other physical (quantum) system, they simulate an abstracted version of themselves.

The computational complexity of both boson sampling and random circuit sampling is well understood and there is a clear exponential gap in run time predicted between the classical and quantum devices. Hence, an experimental demonstration of this can provide evidence for the existence of quantum computers and provide evidence against either the extended Church-Turing thesis, or against complexity theory. The first smallscale experiments appeared around 2013 with only a handful of modes and three or four photons [17-22]. From 2017 and onwards, quantum photonics made a huge steps forward with first a 5 photon and $5 \times 9$ low-loss but not tunable optical network [23]. Two years later, this was already improved to 20 photons and an fixed interferomter of 60 modes in free-space [24]. First experiments with around 50 noisy quantum particles have been reported in recent years for both quantum photonics $[11,25]$ and superconducting qubits $[10,12]$.

Photonics is nowadays one of the leading platforms for quantum computation and quantum simulation since it is the second platform to have a claim on achieving a quantum advantage $[11,25]$. The other claim is from Google's superconducting transmon qubits [10]. Compared to other platforms, photonics has a few distinct advantages. Firstly, single photons behave fully quantum mechanically at room temperature, negating the need for cryogenics. Secondly, photonics has a huge bandwidth compared to electronics. This is especially useful for machine learning [26]. Thirdly, quantum photonics can benefit from developments in the telecom industry, meaning that components developed for telecom can be used for quantum photonics. Finally, even if optical quantum simulators and computers turn out infeasible, quantum optical links and control will still be required. For example, to control trapped ions [27] or for establishing quantum communication links [9]. Hence, technological progress towards optical quantum simulators will always be useful.

Developments in the field of quantum photonics can be divided into two categories. Firstly, there is a constant push towards better hardware that allows generating more and better photons, to improve single-photon detectors and to improve the control of the photon interference for an ever-increasing number of photons. This has now sufficiently matured such that the first companies are founded that are commercializing these technologies. Secondly, the maturing technology is being used for NISQ simulations and proof-of- 
principle experiments. This thesis explores both hardware aspects from a theoretical point, and it explores some initial proof-of-principle experiments. The next sections in this introduction give a more detailed introduction to these fields.

\subsection{The required hardware}

Any possible multiphoton quantum interference experiment that can be thought of, requires three types of hardware. Firstly, a multiphoton source to initialize the quantum state. Secondly, some kind of (non)linear interferometer and thirdly single-photon sensitive detectors. The exact nature of each component can vary from experiment to experiment, but in all cases, the hardware will be similar and comparable.

The lack of universal fault-tolerant optical quantum computers highlights the lack of hardware of sufficient quality. The current development on and towards NISQ-devices are believed to help push state-of-the-art hardware to what is eventually needed for quantum computers and quantum simulators. This section will give a brief overview of the current state-of-the-art hardware of photon sources and interferometers. The description of singlephoton detectors is outside the scope of this thesis.

\subsubsection{Single-photon sources}

Any multiphoton experiment requires a multiphoton source. Multiphoton sources typically consist out of multiple single-photon sources, or a single demultiplexed single-photon source. Regardless of the technology and implementation, there are some universal requirements for multiphoton sources [28].

The first requirement is high photon indistinguishability, which is needed to guarantee the quantum behavior of the photons when they interfere. A second prerequisite is the ability to generate one and only one photon. A third requirement is to generate the photon in a single spatial mode. This is needed when the photons are coupled into a singlemode fiber (SMF). If the spatial mode of the photons is not the standard Gaussian state, then projecting the photons onto the SMF can induce spatial-frequency correlations that reduce the indistinguishability of the photons. Finally, single-photon sources should be as deterministic as possible so that many single photons can be generated simultaneously.

Three smaller requirements for multiphoton sources can be identified. The first is high brightness: so that any experiment has sufficient statistics. The second is that the photons can be generated at telecom wavelength, either 1350 or $1550 \mathrm{~nm}$, as this would enable optimal use of the available telecom technology. The third one is to have a small spectral linewidth. A small linewidth, or long pulse, reduces possible frequencydependent behavior of the optical components and reduces the effect of dispersion in time-bin experiments.

There are a few potential candidates to build a multiphoton source. Traditionally, spontaneous parametric downconversion (SPDC) has been the workhorse of quantum optics [29] and it is still used for leading experiments [11]. SPDC generates pairs of 
photons by exploiting a $\chi^{(2)}$-nonlinear interaction. Operation around $1550 \mathrm{~nm}$ is not only possible, but $1550 \mathrm{~nm}$ is also the ideal wavelength to create indistinguishable photons given the phase-matching properties of the crystal [30]. A limitation of SPDC is that it generates pairs of photons probabilistically, which makes scaling to many photons hard, but not impossible since one photon can be sacrificed to herald the presence of the other $[31,32]$. Despite the widespread use of SPDC, there was concern that in the long run, SPDC is not suitable to generate many single photons of sufficient quality [33-35]. To date, the largest experiment with SPDC used 12 photons [36].

SPDC sources generate in good approximation a single photon when the second generated photon is used as a herald. However, there are cases that use both generated modes of an SPDC source. This type of operation does not generate single photons Fock states, but a thermally distributed Gaussian state. The advantage of Gaussian state generation is that it allows for more input photons or modes for the same number of crystals. The price is that the number of photons remains uncertain. This type of operation has currently the record for the highest number of photons, namely 113 photons generated by 25 nonlinear crystals operated in parallel [11]. However, the computational complexity of Gaussian experiments remain an open question.

A strong competitor to SPDC is quantum dot sources. These sources consist out of an artificial atom, a quantum dot, often inside an optical cavity. When the quantum dots is excited, it typically emits a single photon. Quantum dot sources have proven to efficiently generate many photons at around $700-900 \mathrm{~nm}$ with a narrow linewidth, but only as a single-photon source. The exact properties of each quantum dot vary and subsequently photons from different sources are distinguishable. Thus far, quantum experiments up to 20 photons from a single quantum dot source have been reported [37], even though there are claims over quantum dot devices generating more than 100 photons [38].

A third option is spontaneous four-wave mixing (SFWM) [39]. SFWM is similar to SPDC, but it uses a $\chi^{(3)}$ nonlinearity, meaning that the generation rates will be much lower. However, it can easily be implemented in integrated optics at telecom wavelength, allowing integration of the photon sources with integrated optical networks. This has only been used in smaller, proof-of-principle experiments [40].

\subsubsection{Large optical networks}

In the experiments described in this thesis, the single photons created by SPDC are subsequently sent through a linear optical network. In general, there are requirements for these networks.

The first two requirements are the preservation of photon quality and low loss for the given functionality, which is especially important if the network grows in size. An example of the first requirement is material dispersion in a loop for a time-bin experiment. As the photon travels through the network, it accumulates chirp and hence becomes increasingly distinguishable compared to photons arriving in the time loops at later times. The second requirement is obvious as typically experiments fail when (all) photons are lost. However, the loss is always specified per unit length and the optical length for a certain functionality 
is platform depended. For that reason, the loss per functional unit cell is both a proper metric for comparing systems and engineering target [41].

A third requirement is tunability. Tunability is needed to perform different experiments on the same network and hence makes the network suitable for a variety of experiments. Being able to switch and tune the network at high speeds is especially advantageous as it would allow for measurement-based quantum protocols or fast data processing [42, 43].

There are various methods to implement optical networks. Traditionally, researchers have used free-space optics to implement optical networks. Free-space optics works well for small experiments, but in larger experiments with many modes it will become increasingly difficult to maintain alignment and stability over a prolonged time. Furthermore, the footprint increases quickly to sizes larger than available on optical tables. Therefor it is perhaps surprising that the current largest optical network (144 modes) is entirely constructed with free-space optics. However, this network has about 50\% transmission per photon and is not tunable since the network consists out of many semi silver-coated glass plates glued together [11]. The use of this network is restricted to boson sampling and it remains an open problem if the high losses allow for an efficient classical simulation strategy.

A different approach could be to use multimode systems such as (thin) scattering systems or a multimode fiber. Although such networks also lack tunability, they easily incorporate millions of modes which is unrealistic for any other platform in the foreseeable future. Thick scattering systems are inherently lossy, but a surface scatterer or multimode fiber can provide access to many modes with low losses. Hence, it can be an interesting network for boson sampling and random number generation, at least until other platforms have sufficiently matured.

In the long run, integrated photonics is probably the most promising candidate as it can allow for large, fully tunable networks with a small footprint produced at an industrial scale. Currently, only a few examples have been reported in literature. The largest fully tunable networks reported networks are still around 20 modes, but this number is expected to grow significantly in the foreseeable future. A big advantage is that both photon sources and single-photon detectors can be integrated into such networks [44, 45]. Furthermore, integrated photonics can profit from the ever-going advances of the semiconductor and lithography industry. A small downside is the inevitable absorption and scattering losses in the waveguide when the systems become larger. This might be mitigated by doing measurement-based protocols [43].

\subsection{Proof-of-principle experiments}

It is already possible to perform various types of experiments with the current NISQ hardware. However, these experiments are fully simulable on a standard desktop as the number of photons and modes is limited. Hence, these experiments typically serve two purposes. Firstly, they are proof-of-principle experiments that introduce novel ideas and 
concepts that can later be used for larger systems that are no longer traceable on (standard) classical computers. Note that experiments beyond proof-of-principle are unlikely for NISQ devices [13]. Secondly, these experiments highlight the quality of the current stateof-the-art technology. In other words, not the signal but the (lack of) noise is what is important.

The following sections introduce a few experiments that are described later in this thesis. All experiments are carried out on a fully tunable 12-mode interferometer with a transmission of $\sim 60 \%(2.2 \mathrm{~dB})$ per photon [46].

\subsubsection{High fidelity control over the network}

The high control fidelity over the network is an obvious requirement for larger networks and quantum experiments. But a large, well-controlled network with proper access to all input and output channels is a platform that can be used for much more than quantum optics alone. An example is a long-standing open problem of wave transport through disordered media, such as electron transport through nanowires, photon transport to scattering media or multimode fibers, and phonon transport $[47,48]$. In all these types of wave transport, one can ask how the transmission efficiencies are distributed for all transmission eigenchannels of the network. Transmission eigenchannels are the eigenmodes of the transmission matrix that describes the scattering system. One would naively expect that those transmission efficiencies are clustered around its mean, e.g. such as in a Gaussian distribution. However, this has been proposed that these transmission efficiencies are distributed in a bimodal distribution [49, 50].

Despite considerable experimental effort, this distribution has not yet been experimentally shown. It turned out that the problem preventing the observation of the bimodal distribution was the need to properly access all input and output modes of the transmission matrix [51-53]. This is no simple feet for electrons -because of their small size- or photons -because of limited NA in any experiment. A large, fully tunable network with well-defined modes, such as a tunable integrated photonic interferometer as needed for boson sampling can be used to experimentally observe the bimodal distribution.

\subsubsection{Certification of entanglement}

Certifying a quantum experiment that is beyond the capabilities of current classical computers to simulate, is a nontrivial problem since it is not possible to compare the experimental results with a numerical simulation. A good example of when this is problematic is boson sampling, which is designed to be intractable for classical computers [15]. Hence, certifying that the boson sampling experiment succeeded requires statistical analysis of a limited data set that can be obtained and analyzed in polynomial time [22, 54-56].

In a more general case, it is important to be able to certify multiphoton entanglement in a single measurement with a computationally efficient data analysis and realistic resources. Such a tool would allow to probe whether the input photons indeed interfered with each other and whether the photons remained indistinguishable from each other. The latter is 
by no means trivial as, for example, small path length differences in a large network can quickly accumulate to a path length difference larger than the photon's coherence length.

\subsubsection{Thermalization of pure quantum states}

The last possible experiment explored revolves around a paradox between quantum and thermodynamics: the thermalization of a quantum state in an isolated system. The underlying problem of thermalization is the paradox that the global entropy of an isolated quantum state remains zero overall unitary (energy conserving) transformations, while thermalization is known to occur since it maximizes the entropy. So how can a welldefined quantum state thermalize in a closed, isolated system?

This paradox is resolved by realizing that local observers lack knowledge of global correlations [57]. It has been suggested that global entanglement drives local entropy maximization, i.e., local thermalization is the result of global quantum entanglement. So far this has been experimentally tested with cold atoms in an optical lattice [58]. These cold atoms experience a Bose-Hubbard interaction inside the optical lattice, which causes them to thermalize. However, the nature of these experiments is inherently irreversible as it is required to measure after a specific time $\tau$ to observe the thermal distribution.

A more powerful approach is to test the thermalization on a quantum system that is reversible. For this, the time needs to be simulated. This is possible by realizing that the time evolution is nothing but a unitary transformation of the Hamiltonian $H$ acting on the entire system:

$$
U=\mathrm{e}^{-i H \tau}
$$

where $U$ denotes the unitary time evolution. This approach does not only allow to dial certain time, but it also allows to undo the time evolution with the inverse of $U$. By undoing the time evolution, one can verify that the quantum particles have not yet become entangled with the experimental apparatus, which is otherwise challenging. By the process of elimination, one must conclude that the thermalization must arise from local entanglement if the quantum particles show thermal distribution when they are entangled and if these particles are shown not to be entangled with the environment, i.e. the experimental apparatus.

\subsection{Thesis outline}

This thesis explores all aforementioned themes that are relevant for multiphoton interference. The first theme of the thesis are possible high-dimensional networks for multiphoton interference. These are chapters 2 and 5. The second theme revolves around single-photon sources, both theoretical limitations of SPDC and a characterization of our multiphoton SPDC setup for telecom wavelengths. These are chapters 3 and 4 . Finally, the third theme combines the first two by showing three integrated quantum-optical experiments. These are described in chapters 6,7 and 8 . 
Chapter 2 explores a different way of realizing a low-loss and high-dimensional network, namely via surface scattering. Specifically, the use of boson sampling is considered. This solves the current open problem in boson sampling [11] by combining access to thousands of optical modes while maintaining low losses. Even though the surface scatterer is not universal, it still allows the implemention of boson sampling and hence functions as a benchmark for the advancement of single-photon sources and single-photon detectors.

Chapter 5 describes and characterizes a more conventional method to implement optical networks, namely integrated photonic waveguide networks. This photonic processor is realized in silicon nitride and has a record number of 12 input and 12 output modes that are all coupled to each other. Furthermore, the network is fully tunable. The extend of the tunability is investigated and the preservation of the quantum nature of single photons is studied.

Chapter 3 explores the fundamental limits of SPDC sources for boson sampling from a computational complexity point of view. Thus far, the optimal trade-off between photon imperfections was not known. Using the latest theoretical developments it became possible to find the trade-off between the main imperfections, namely loss and distinguishability [59]. This framework gives a lower limit to the number of photons that interfere quantummechanically for a given set of imperfections. This allows optimizing the photon sources explicitly for the computational complexity of boson sampling.

Chapter 4 continues on the work of Chapter 3: it describes and characterizes a current four-photon SPDC source and all related hardware. This description of the setup is the foundation of the third and last part of the thesis: the proof-of-principle experiments.

Chapter 6 solves a long-standing open problem of wave transport through disordered media, namely the consequence of open scattering channels on transmission eigenvalues. This chapter does not only show the predicted distribution, it also confirms the theoretical predictions that full access to all input and output modes are necessary to observe this distribution [51-53]. On a more abstract level, this chapter does not only show that integrated optics can be used to study different types of physics, it also marks the point that integrated optics is sufficiently developed to actually perform such experiments.

Chapter 7 shows a computationally efficient protocol to certify multiphoton distinguishability in a tunable network by means of a simple two-mode correlator [55]. For this, a matrix is found that maximizes this two-mode correlator when (partial) indistinguishable photons are injected. The notion that this matrix is optimal in the space of all matrices is important, since it implies the protocol to be one-sided device-independent. That is, the hardware -the interferometer- does not have to be trusted to implement the correct matrix. When the device indeed implements the ideal matrix incorrectly, then this will inevitably reduce the two-mode correlator. Hence the two-mode correlator is guaranteed to give a lower bound to the certification of distinguishability.

Chapter 8 explores the thermalization of a quantum state in a closed system: an integrated photonic processor. This experiment shows that local thermalization is the result of global entanglement with the unobserved remaining universe. The experiment is also reversible since the integrated processor allows to directly implement any unitary transformation on the input state. Reversibility is useful as it eliminates the need for 
homodyne and heterodyne detection is to certify if the global state generated by the network has indeed thermalized [60].

Finally, Chapter 9 contains a summary of all results in this thesis and is complemented with the outlook for possible future directions. 


\section{Boson sampling in low-depth optical systems}

Recently, the world has seen the first claims of a demonstration of a quantum advantage [10-12, 25], i.e. the first systems in which a quantum computer outperforms a classical one at some specific, well-defined computational task. These systems represent a major milestone on the road toward applications of quantum computing for tasks beyond those that can be carried out with classical computers.

Boson sampling is a protocol to demonstrate a quantum advantage using quantum interference in linear optics [15]. In boson sampling, $n$ indistinguishable photons are created at photon sources and sent through a linear optical network whose transformation matrix is chosen uniformly according to the Haar measure. The task is to generate samples from the output distribution of this interferometer when measured in the Fock basis. For a sufficiently large optical system, the probability distribution is given by permanents of matrices that are very well approximated by identical and independently distributed (i.i.d.) Gaussian elements. This distribution is believed to be hard to sample from for a classical computer, with the computational cost scaling as $n 2^{n}$ for the best-known classical algorithms [61], which places the boundary of a quantum advantage at $n \approx 50$ photons [62]. At the same time, a linear optical network can natively implement this computational task through multi-photon interference and thereby generate samples from the target output distribution efficiently.

Since this protocol was proposed in 2011 , there has been major progress on its experimental implementation. This started with smaller-scale boson sampling experiments [18-21, 24, 36, 63, 64] to the current state of the art experiments, which consists of a 144 mode Gaussian boson sampler [32, 65] with 25 two-mode squeezed photon sources [11].

The challenge in carrying out these experiments is to adhere as closely as possible to the original boson sampling protocol, and not introduce any imperfections, which may give rise to classical simulability. The general physical picture is that noise degrades the level of quantum interference in these systems, opening up the way for either a direct simulation [59, 66-71], or spoofing a benchmark test [72, 73].

The strongest imperfection in large-scale boson sampling experiments is photon loss, which is an imperfection where not every photon that is generated in the sources ultimately results in a detection event in one of the detectors. For all proposed variants of boson sampling, loss is an imperfection that degrades the strength of quantum interference, although only for some variants it is known how to use this fact to construct an efficient classical simulation [69]. Finding ways of improving the optical transmission of boson sampling experiments is therefore an urgent problem. This is especially true since, in all current implementations of boson sampling, the number of optical components that must be traversed grows linearly with the width of the optical circuit, and hence the optical loss grows exponentially with the circuit size [67].

The content of this chapter is adapted from the following submitted paper: R. van der Meer et al., arXiv:2110.05099. 
a)

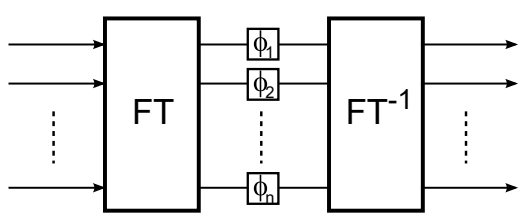

b)

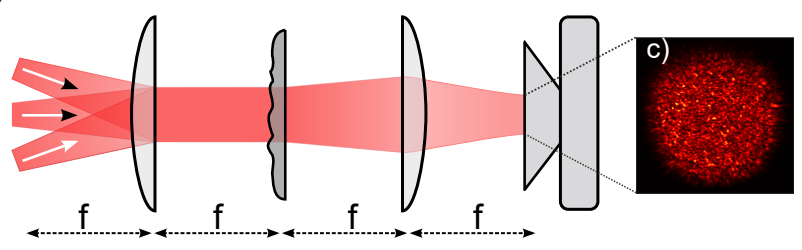

Figure 2.1 The network. A schematic of the proposed network with 4 input modes. a) The mathematical description of the physical apparatus as shown in b). The apparatus consists of two lenses and a thin, rough surface in between the lenses. The random interference pattern as observed on the detector (c) is known as speckle.

Another imperfection that plays a role in large-scale boson sampling experiments is detector blinding, which occurs when the number of modes $m$ is comparable to the number of photons $n$, meaning that a significant fraction of photons will end up in identical output modes, which is termed a collision. In this situation, if threshold detectors are used at the outputs, some photons are not detected, which constitutes an imperfection that can be used for simulation [74]. In order to avoid collisions, the number of optical modes must scale as $m \propto n^{2}$, a scaling that is typically not obeyed in experiments $[11,25,75]$.

In this chapter, we present a simultaneous solution to the problems of optical loss and detector blinding by proposing a constant-optical depth circuit that is scalable to the required thousands of optical modes. This circuit can be realized in the form of a thin optical scatterer (e.g. a ground glass plate) placed in a $4 f$-configuration in the simultaneous focal point of two lenses (see Fig. 2.1). We make use of the fact that a lens implements a Fourier transform on an almost arbitrary number of optical modes - up to 1 million modes per $\mathrm{mm}^{2}[76]$ - to conveniently realize a network with three components and constant optical loss in the system size. When a Fourier transform is implemented as pairwise mode interactions, e.g. in integrated optics, the number of required interactions per mode in the circuit scales as the logarithm of the number of modes involved. [77, 78]. This platform is a simplified version of an universal method to implement any arbitrary transfer matrix [79]. We estimate that the overall efficiency of this protocol will be $85-90 \%$, which would be sufficient to defeat all currently known classical simulation algorithms for boson sampling.

The price of this low-tech implementation is a complete loss of reconfigurability of the optical system. Furthermore, the fact that our scattering system scrambles the phase of each incoming k-vector in an identical way induces an additional constraint to the transmission matrix $U$, namely that it must be of circulant form, i.e. that each row of the sampling 
matrix is a copy of the previous row shifted by one entry. These additional correlations in the sampling matrix could prima facie affect the computational hardness of the protocol. The bulk of this work is concerned with presenting a combination of numerically and theoretical results which provide evidence that these additional correlations disappear in the appropriate limits of large numbers of photons and modes, thereby making our system suitable as a low-loss implementation of boson sampling. We name our proposal circulant boson sampling after the circulant nature of the scattering matrix.

Our work is organized as follows: we start in Section 2.1 with some physical arguments based on concepts from scattering theory. Then, Section. 2.2 formally introduces the circulant boson sampling and identifies the problems which need to be solved to guarantee the computational complexity. Section 2.3 gives an informal overview of the main results on demonstrating the computational complexity. Sections 2.4 and 2.5 show the detailed results in which we show how circulant boson sampling maintains computational complexity. Finally, Section 2.6 addresses the technical feasibility of the system and focuses on the required hardware.

\subsection{Thin scattering systems}

In this section, we will use some concepts from scattering theory to build up physical intuition for circulant boson sampling. For this, we start with a short description of thick scattering systems and then introduce the circulant network.

Perhaps counterintuitively, disorder is a desirable feature in many classical optical systems, where a high number of independent optical modes are needed. An example is in microscopy, where a diffusor is used to convert a point source into quasi-uniform illumination. Functionalizing disorder to achieve new optical applications is an active field of research $[47,76,80]$.

A crucial factor that determines the properties of a scattering system is how often light is scattered while traversing the medium. Everyday optical systems in which scattering plays a role are typically multiple-scattering ('thick') systems, examples of which are white paint and milk. In multiple-scattering materials, incident light scatters many times on average before it diffuses out of the material. Repeated scattering events collectively scramble the polarization of the incoming light and effectively erase all directionality, i.e., the light is scattered in all $4 \pi$ directions.

Even in thick optical samples, the output distribution pattern is not uniform when pumped with coherent light. The random paths which the light takes through the sample are coherent and hence undergo interference. This random interference leads to a pattern of spatial intensity variations, which is referred to as speckle. An example of speckle is shown in Fig. 2.1b).

The properties of speckle patterns have been extensively studied [48, 76, 81]. A key property of speckle is its high degree of randomness. Random interference of many independent optical paths causes a complex Gaussian distribution of the elements of the scattering matrix. This is observed experimentally through the Rayleigh distribution of 
the optical field strengths leading to an exponential distribution of the intensities of the speckle pattern $[48,81,82]$. In the limit of thick optical systems, small submatrices of the scattering matrix approach an i.i.d. Gaussian distribution, as the scattering effectively erases all structure and only a part of all transmission modes can be observed. The fact that the matrix elements are i.i.d. Gaussian distributed turns out to be precisely the requirement for the computational complexity of boson sampling [15].

Unfortunately, boson sampling also requires a low-loss optical system, and for this, thick scattering systems are not suitable [83-85]. The ultimate reason for this is that in a multiple-scattering system the light is scattered over a $4 \pi$ solid angle, which makes a collection of all the light scattered from the sample experimentally infeasible.

The network proposed in Fig. 2.1, which uses a single thin scattering layer, can be thought of as a system that combines the high degree of randomness of a thick scattering system with low optical loss. Such a system still grants access to a large number of modes, but at the same time reduces the losses since all the light will be transmitted over a small spread of spatial angles [81].

Unfortunately, having only one layer of scatterers does not allow for the same degree of mode mixing as is the case in thick scattering media. The consequence of this is that while the individual matrix elements in the scattering matrix all follow a Gaussian distribution (in the language of scattering theory 'exhibit fully developed speckle') [86], they exhibit significant correlations (in the language of scattering theory: they strongly exhibit a memory effect), and it is not given that the distribution of submatrices of such a matrix approaches an i.i.d. Gaussian distribution. It is precisely these additional circulant correlations that are the subject of this work.

\subsection{Boson Sampling}

In this section, we will first introduce boson sampling. Then, we will introduce a few key arguments from its original proposal [15] regarding the hardness of boson sampling, followed by our proposal for a modified protocol based on circulant matrices. A comprehensive and more in-depth discussion on boson sampling can be found in [87].

In a boson sampling experiment, $n$ indistinguishable photons are injected in $n$ distinct input modes of an $m \times m$ random lossless interferometer $(m>n)$. The photons interfere as they propagate through the interferometer, resulting in an output probability distribution comprised of $\left(\begin{array}{c}m+n-1 \\ n\end{array}\right)$ entries.

Assume without loss of generality that the photons are incident on the first $n$ input modes of the system, such that the input wave function is given by:

$$
\left|\psi_{\text {in }}\right\rangle=|1\rangle^{\otimes n}|0\rangle^{\otimes m-n},
$$

and let the transmission through the interferometer be described by a complex unitary 
matrix $U \in \mathbb{C}^{m \times m}$ drawn from the Haar measure. The output state is then given by:

$$
\begin{aligned}
\left|\Psi_{\text {out }}\right\rangle= & U\left|\psi_{\text {in }}\right\rangle \\
& \sum_{\mathrm{s}} P_{\mathrm{s}}\left|n_{o, 1}^{(\mathrm{s})}, n_{o, 2}^{(\mathrm{s})}, \ldots, n_{o, m}^{(\mathrm{s})}\right\rangle,
\end{aligned}
$$

with $s$ a particular output configuration and $P_{\mathrm{S}}$ its corresponding probability amplitude [88]. Finally, $n_{o, m}^{(\mathrm{s})}$ denotes the number of bosons in the output channel $m$ given this configuration s. The probability associated with a certain outcome $P_{\mathrm{s}}$ is given by the interference of how the $n$ input photons can end up at that specific output configuration $\mathrm{s}$. This is given by the permanent of an $n \times n$ submatrix $U_{\mathrm{T}}$ relating the used input modes to the output configuration $[15,88]$

$$
P_{\mathrm{s}}=\frac{\operatorname{perm}\left(U_{\mathrm{T}}\right)}{\sqrt{n_{o 1}^{(\mathrm{s})} !, \ldots, n_{o m}^{(\mathrm{s})} !}},
$$

with the submatrix $U_{\mathrm{T}}=U_{\rho, s}$, with $\rho$ the input configuration as defined in Eq. 2.1. The denominator is a normalization term and perm denotes the permanent. The permanent is defined as:

$$
\operatorname{perm}(U)=\sum_{\sigma \in S_{n}} \prod_{j=1}^{n} U_{j, \sigma(j)},
$$

with $\sigma$ summing over the set of all permutations $S_{n}$.

The computational complexity of boson sampling ultimately derives from the conjecture that approximate sampling over the probability distribution given by $\left|P_{\mathrm{S}}\right|^{2}$ is computationally hard, if $U_{\mathrm{T}}$ is given by a matrix of i.i.d. Gaussian entries. The intuition behind the choice of this family of matrices is that i.i.d. Gaussian matrices have no internal structure which a classical algorithm could exploit to achieve a speedup.

For the matrices $U_{\mathrm{T}}$ to be close to i.i.d. Gaussian, two conditions must hold. First, any $n$-by- $n$ submatrix of $U$ must converge to a matrix of i.i.d. Gaussians in the limit of a large number of modes. The intuition here is that as the submatrix becomes smaller with respect to the overall matrix $U$, the unitary constraint becomes less dominant, removing any correlations between the matrix elements. Aaronson and Arkhipov were able to show that if $U$ is drawn uniformly according to the Haar measure, then for a scaling of $m \propto n^{5} \log ^{2} \mathrm{n}$, an $n$-by- $n$ submatrix is close to i.i.d. Gaussian.

The second requirement is that the output distribution must actually be made up of $n$-by- $n$ matrices drawn from $U$, avoiding repeated rows or columns in $U_{\mathrm{T}}$. These arise when photons emerge from the same optical mode, which is termed a collision. The probability of collisions must be made arbitrarily small, a condition which is satisfied for Haar-random matrices when $m \propto n^{2}$.

To argue the hardness of our proposed protocol, we must show that these two conditions hold (perhaps with different polynomial scaling in the number of modes) for our proposed family of sampling matrices. Our goal in what follows will be to provide evidence that the intuition from sampling over Haar-random matrices holds also for circulant matrices, 
namely that the additional correlations which are introduced by the constraints on the matrices decay when the number of modes is made large enough with respect to the number of photons.

In our protocol, the only change we make compared to the original proposal [15] is the family of optical circuits over which we sample. Instead of Haar-random matrices, we now sample over matrices of the form $U_{\mathrm{c}}=F \Phi F^{-1}$, where $F$ is an $n$-by- $n$ Fourier matrix with the appropriate normalization and $\Phi$ is a diagonal matrix whose entries are given by $\Phi_{j j}=\exp \left(i \phi_{j}\right)$, with each $\phi_{j}$ chosen independently on the interval $[0,2 \pi]$. The resulting matrix has the form:

$$
U_{c}=\left[\begin{array}{cccc}
c_{1} & c_{2} & \ldots & c_{m} \\
c_{m} & c_{1} & \ldots & c_{m-1} \\
\vdots & \vdots & \ddots & \vdots \\
c_{2} & \ldots & \ldots & c_{1}
\end{array}\right]
$$

with $c_{j} \in \mathbb{C}$, and is called a circulant matrix. Note that this matrix has only $m$ unique elements since each row or column is a repetition of the previous one where all elements have been shifted by one position. These additional correlations are an additional obstacle in letting the elements of a truncated matrix $U_{\mathrm{T}}$ approach i.i.d. Gaussians.

\subsection{Overview of results}

In this section, we provide a general overview of our results. Our strategy to argue the computational complexity of circulant boson sampling is to closely follow the proof for standard boson sampling [15]. To this end, we must show that (1) truncations of circulant matrices should approach i.i.d. Gaussians with polynomial scaling in $n$ and $m$ and (2) that there is a polynomial scaling between $n$ and $m$ in which the no-collision condition holds. In this section we provide an informal overview of the main results on these two points. For both points, we rely on a combination of numerical and analytic results to provide our evidence.

A crucial fact about our system is that the output configurations are not equiprobable, unlike the Haar-random case [89]. This is due to the fact that repeated entries in the sampling matrix give rise to higher order moments of matrix elements in the expected value of the permanent. This fact means that the symmetry arguments employed in $[15,89]$ are not available to us.

Truncations - analytical result We show that the variation distance $\Delta_{C}$ between the probability distribution of submatrices of circulants and the i.i.d. Gaussian ensemble is upper bounded by

$$
\Delta_{\mathrm{C}} \leq \dot{C} \frac{n^{2}}{\sqrt{m}}
$$

For some universal constant $\dot{C}>0$.

Truncations - numerical results We provide evidence that the eigenvalue spectrum of a truncated circulant matrix strongly resembles that of a truncated Haar-random matrix. 
In particular, we provide numerical evidence that the fidelity between the eigenvalue distribution of a Gaussian and truncated circulant matrix remains constant at $m \propto n$ scaling.

Furthermore, we provide evidence that the convergence of permanents of truncated circulant matrices to permanents of i.i.d. Gaussian matrices have the same scaling as the convergence of permanents of truncated Haar matrices to permanents of i.i.d. Gaussian matrices. This is another, independent piece of evidence for the convergence of submatrices of circulant matrices to i.i.d. Gaussians.

No collisions - Analytical results Collisions in the matrix elements reduce the computational complexity of boson sampling. The original proposal uses Haar-random matrices, where every photon 'blocks off' a single potential output mode for the remaining photons (namely the one which it occupies). In circulant boson sampling, the cyclic nature of circulant matrices means that each photon blocks off $n$ adjacent modes as well.

We show that if we assume all outcomes in the boson sampler are equiprobable averaged over all circulant matrices (which is only approximately true for circulant matrices), a scaling of $m \propto n^{3}$ is required to achieve a constant probability of an outcome with repeated elements in the matrix (i.e. a collision).

No collisions - numerical results We use a Monte-Carlo simulation to provide evidence that the $m \propto n^{3}$ scaling inferred from our simplified calculation assuming equiprobable outcomes also holds when we take into account the fact that not all outcomes are equiprobable.

\subsection{Truncations approach i.i.d. Gaussian}

In this section, we provide evidence that sufficiently small truncations of circulant matrices become close, in variation distance, to a matrix with i.i.d. Gaussian elements. To this end, we follow the strategy of the original boson sampling proposal [15]. Their approach has two steps. Firstly, they showed that a polynomially small submatrix of a larger, Haarrandom unitary matrix becomes approximately Gaussian distributed. Then this result was used to prove the stronger statement, namely the multiplicative bound

$$
p_{S}(X) \leq(1+O(\delta)) p_{G}(X)
$$

$\forall X \in C^{n \times n}$ and with $p_{S}$ and $p_{G}$ the probability density functions of Haar random unitaries and i.i.d. Gaussians, respectively.

In the following subsections, we will first show that the submatrix of a circulant matrix approaches the i.i.d. Gaussian ensemble additively. Unfortunately, we were unable to prove the multiplicative bound from the additive bound. As additional evidence of convergence, we study both the eigenvalue spectra of truncated circulant matrices and the average outcome of permanents of truncated circulant matrices. Both approaches independently provide insight to the convergence to i.i.d. Gaussians. 


\subsubsection{Analytical results}

We first present our analytical results providing evidence that the distribution of $n$-by- $n$ submatrices of non-identical elements (i.e. without repetitions in the elements) converges to an i.i.d. Gaussian distribution.

Rather than looking at a submatrix, we can limit ourselves to a single vector containing all elements of the submatrix. The vector has a length of $d=m^{2}$ and thus corresponds to the submatrix. The goal is to show that the effect of the unitary constraint on this vector becomes negligible for some suitable scaling of the length of the vector. For this first step, we use theorem 1.1 of [90].

Theorem 1. Let $\mathbb{R}^{n}$ be a real Euclidian $n$-dimensional space of vectors $x=\left(x_{1}, \ldots, x_{n}\right)$ with the norm $\|x\|_{2}^{2}=\sum_{i=1}^{n} x_{i}^{2}$ and the scalar product $\langle x, x\rangle=\|x\|_{2}^{2}$. Let $X_{1}, \ldots, m$ be independent random vectors with a common mean $E(X)=0$. Write $S=\sum_{j=0}^{m} X_{j}$. Throughout we assume that $S$ has a degenerated distribution in the sense that the covariance operator, say $\Sigma=\operatorname{Cov}(S)$, is invertible. Let $Z \sim N(0, \Sigma)$ be a Gaussian random vector with the same covariance matrix as that of $S$. Let $C$ be the class of all convex subsets of $\mathbb{R}^{n}$. Then there exists a universal constant $\mathcal{C}>0$ such that

$$
\begin{aligned}
\Delta_{\mathrm{C}} & :=\sup _{A \in C}|\operatorname{Pr}\{S \in A\}-\operatorname{Pr}\{Z \in A\}| \\
& \leq \mathcal{C}^{\frac{1}{4}} \sum_{j=1}^{m} E\left(\left\|\Sigma^{-\frac{1}{2}} X_{j}\right\|_{2}^{3}\right),
\end{aligned}
$$

where $\Sigma^{-\frac{1}{2}}$ stands for the positive root of $\Sigma$.

We now show how we can apply this Theorem to the case where we pick $n$ elements of a vector of length $m$ which is the Fourier transform of a vector of random phases. Let $\Phi_{1}, \ldots, \Phi_{n}$ be i.i.d. uniform on $[0,2 \pi]$. Fix $k_{1}, \ldots, k_{m} \in\{1, \ldots, d\}$. For $j=$ $0, \ldots, d-1$, define $X_{j}^{(m)}$ to be the vector containing all matrix elements

$$
\begin{aligned}
X_{j}^{(m)}= & \frac{1}{\sqrt{m}}\left[\begin{array}{c}
w_{m}^{j k_{1}} e^{i \phi_{j}} \\
\vdots \\
w_{m}^{j k_{n}} e^{i \phi_{j}}
\end{array}\right] \\
= & \frac{1}{m}\left[\begin{array}{c}
\cos \left(\phi_{j}+2 \pi \frac{j k_{1}}{m}\right) \\
\sin \left(\phi_{j}+2 \pi \frac{j k_{1}}{m}\right) \\
\cos \left(\phi_{j}+2 \pi \frac{j k_{2}}{m}\right) \\
\vdots \\
\sin \left(\phi_{j}+2 \pi \frac{j k_{m}}{m}\right)
\end{array}\right] \in \mathbb{R}^{2 n},
\end{aligned}
$$

where the first line is a column vector whose elements are given by a discrete Fourier transform applied on the vector of phases $\phi_{j}$, and $w_{m}^{j k_{l}}=\frac{1}{\sqrt{m}} \exp \left(\frac{-2 \pi i j}{m} k_{l} l\right)$. The second 
line expands the space by a factor two by splitting up the real and imaginary part of the previous line.

The $X_{j}^{(m)}$ are independent and clearly, $E\left(X_{j}^{(m)}\right)=0$ for all $j=0, \ldots, m-1$. Furthermore, it can be checked that (under some assumptions on $k_{1}, \ldots, k_{n}$, see [91])

$$
\operatorname{Cov}\left(\sum_{j=0}^{m-1} X_{j}^{(m)}\right)=\frac{1}{2} I_{2 n}
$$

This covariance matrix is clearly invertible, hence we can apply Theorem 1 to obtain:

$$
\begin{aligned}
\Delta_{\mathrm{C}} \leq & \mathcal{C} n^{\frac{1}{4}} \sum_{j=0}^{m-1} E\left(\left\|\left(\frac{1}{2} I_{2 m}\right)^{-\frac{1}{2}} X_{j}^{(d)}\right\|_{2}^{3}\right) \\
= & 2^{\frac{3}{2}} \mathcal{C} n^{\frac{1}{4}} \sum_{j=0}^{m-1} E\left(\left\|X_{j}^{(m)}\right\|_{2}^{3}\right) \\
= & 2^{\frac{3}{2}} \mathcal{C} n^{\frac{1}{4}} \sum_{j=0}^{m-1} \\
& E\left(\sqrt{\frac{1}{m}\left(\sum_{l=1}^{n} \cos ^{2}\left(\phi_{j}+2 \pi \frac{j k_{l}}{m}\right)+\sin ^{2}\left(\phi_{j}+2 \pi \frac{j k_{l}}{m}\right)\right)}\right) \\
= & 2^{\frac{3}{2}} \mathcal{C} n^{\frac{1}{4}} \sum_{j=0}^{m-1} E\left(\frac{n^{\frac{3}{2}}}{m^{\frac{3}{2}}}\right)=2^{\frac{3}{2}} \mathcal{C} n^{\frac{7}{4}} m^{-\frac{1}{2}} \\
\leq & \mathcal{C} \sqrt{\frac{n^{\frac{7}{2}}}{m^{m}}} .
\end{aligned}
$$

For a universal constant $\mathcal{C}>0$. In other words, the unitary constraint is upper bounded by $\frac{n^{2}}{\sqrt{m}}$.

This result gives evidence that the number of modes must scale as $m \propto n^{4}$ to hide the unitary constraint. This shows the submatrix-of-circulant approaches the i.i.d. Gaussian ensemble additively. Unfortunately, unlike the case of Haar-random matrices, there appears to be no easy way to use the additive bound to prove the multiplicative bound.

\subsubsection{Eigenvalue spectra}

In the original boson sampling proposal, the multiplicative bound was found by examining the eigenvalue spectra. [92-95]. Inspired by this, we carry out a Monte-Carlo simulation of the eigenvalue spectra. To this end, $10^{6}$ truncated matrices of size $n$-by- $n$ are obtained, each from another Haar-random unitary and circulant unitary matrix of size $m$-by- $m$. Each truncation is chosen such that repeated entries are absent, i.e., it implicitly assumes an outcome without collisions. To quantify the similarity of the two distributions $A$ and $B$, 

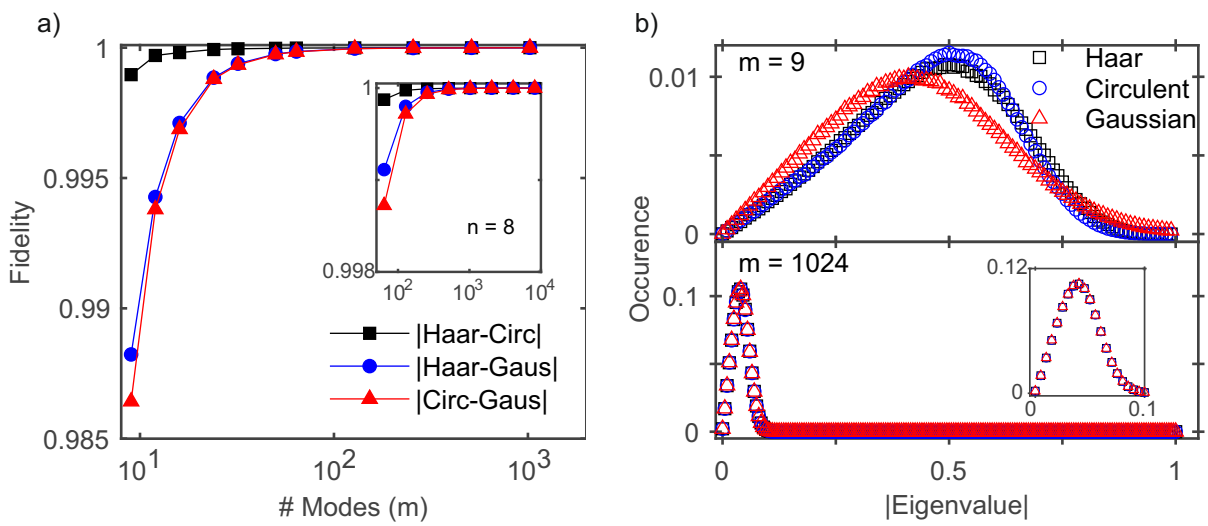

Figure 2.2 Fidelity with i.i.d. Gaussians. a) The convergence of the fidelity between eigenvalue distributions in the case of 3 photons. The inset shows the case for 8 photons. b) Two examples of the eigenvalue distribution for 3 photons when $m=9$ and $m=1024$. The inset on the bottom right is a zoom-in on the peak of the latter.

we use the fidelity as given by: [6]

$$
F(A, B)=\sum_{j=1}^{n} \sqrt{P(A)_{j} P(B)_{j}},
$$

where $P(X)_{j}$ denotes the probability of the $j^{\text {th }}$ eigenvalue. Note that this Monte-Carlo approach can only provide insights to the average fidelity between the eigenvalue spectra, it does not lower-bound the minimum fidelity.

We start with a system of fixed photon number $n=3$, where we show how the fidelity of truncations of both Haar-random unitaries as well as circulant matrices with the i.i.d. Gaussian ensemble approach unity as the number of modes is increased. Figure 2.2a) shows how the fidelity depends on the number of modes $m$ at a fixed photon number. Despite the differences between the truncated-Haar and truncated-circulant matrix ensembles, it is striking how similar their approach to $F=1$ is as the number of modes is increased. That the distributions are indeed close is confirmed by the black points in Fig. 2.2a), which show the fidelity between the Haar and circulant distributions, which show that they resemble each other more than that either resembles the i.i.d. Gaussian ensemble. The inset shows that this behavior remains when the number of photons is increased, in this case to $n=8$. The similarity of the fidelities as shown in 2.2a) is illustrated by the two example eigenvalue distributions shown in Fig. 2.2b). Again, the truncations of both the Haar-random and the circulant matrix are similar and in the limit of $m \gg n$ clearly converge to that of i.i.d. Gaussians.

So far, we have considered eigenvalue distributions of truncated matrices at a fixed number of photons $n$ as a function of the number of modes. The next thing to show is how the ratio of modes and photons should scale for a fixed fidelity. This is shown in Fig. 


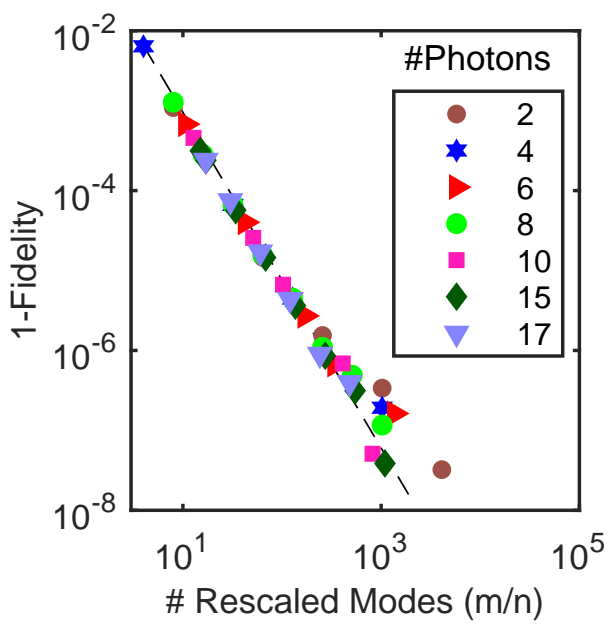

Figure 2.3 Convergence of fidelity. Convergence of the fidelity between circulant and Gaussian truncations with a rescaled $\mathrm{x}$-axis. The dashed line is a guide to the eye.

2.3, where the convergence of the fidelity between the circulant and Gaussian truncation is plotted as a function of the number of modes $m$ for a different number of photons $n$. The $\mathrm{x}$-axis is rescaled by the number of photons in order to emphasize the similar trend of the fidelity for all photons. The graph suggests that the number of modes should scale as $m \propto n$, however, note that this is the result after averaging over the space of circulant matrices; it is not the worst-case scenario. The deviation from the dashed line for a small number of photons is the result of using a finite number of samples in the simulation. This effect is more profound for small $n$ as each random matrix only contributes $n$ eigenvalues to the histogram.

\subsubsection{Permanents}

Another, more direct way to investigate the effect of the circulant correlations on the convergence to i.i.d. Gaussians is to look at the average outcome of permanents of submatrices of Haar-random and circulant matrices. If the correlations disappear with some suitable scaling of the number of modes and photons, the expected value of the permanent of a submatrix of a circulant $C$ must approach that of the i.i.d. Gaussian ensemble $\mathrm{X}$, which has $E\left(|\operatorname{Perm}(X)|^{2}=n ! / m^{n}\right.$. Having the average outcome of a permanent of a submatrix of a circulant matrix approach this value is a necessary but not a sufficient condition for the ensemble to approach that of i.i.d. Gaussians, but it can serve to provide numerical evidence.

Figure 2.4 shows how the distance between the expectation values of truncations of both a Haar-random unitary and a circulant unitary with the result of an i.i.d. Gaussian scale with the number of modes, $m$. Each data point is the mean of $10^{6}$ random instances 


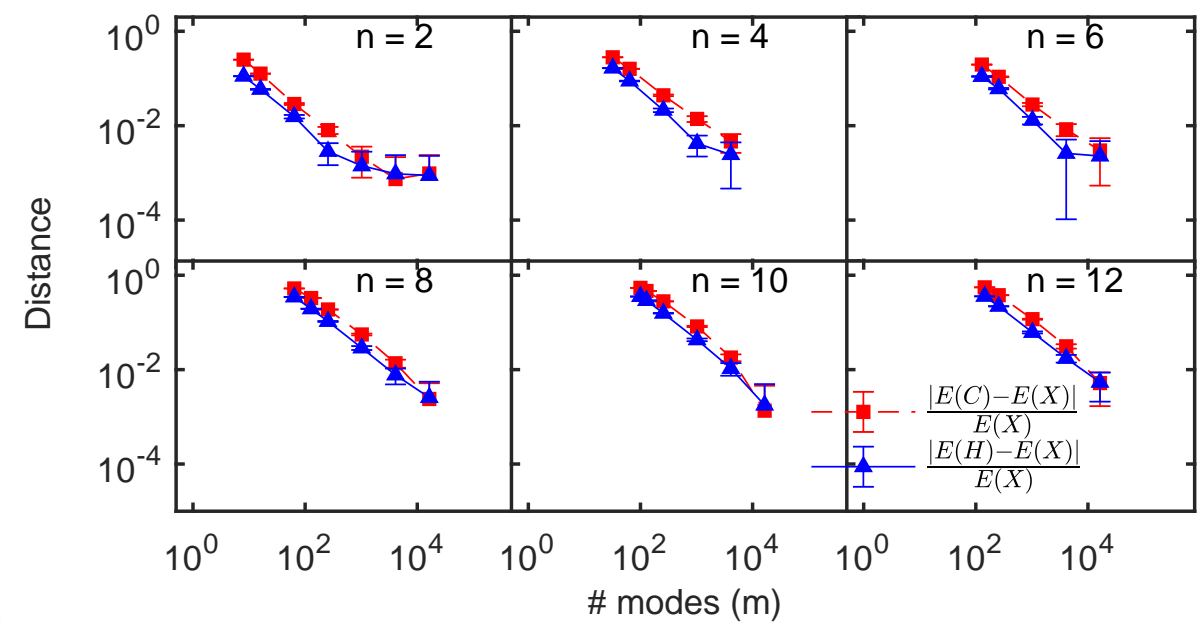

Figure 2.4 Convergence of permanents. Numerical evidence for the convergence of the average permanent for a various number of photons $n$ as a function of the number of modes $m$. The red squares show how the distance between the average permanent of truncated circulant matrices and the result of i.i.d. Gaussian matrices diminishes with $m$ and the blue triangles show the same for truncated Haar matrices.

and the error bars denote the standard deviation. What is notable is that for each data point where we have sufficient numerical resolution in our simulation (as indicated by the error bars), the distance between Haar and Gaussian and between circulant and Gaussian differs only by a constant value as the number of modes is increased.

This result reinforces the picture from our study of the eigenvalue spectrum, namely that the submatrix-of-circulant distribution strongly resembles the submatrix-of-Haar distribution, which is known to converge to i.i.d. Gaussian in the appropriate limits. A full proof would require a multiplicative version of the result of section 2.4.1.

\subsection{No-collisions}

The second prerequisite for our protocol is to show that the probability of observing collisions (i.e. outcomes with repeated matrix elements) becomes small with appropriate scaling of the number of modes $m$ versus the number of photons $n$. In the case of a Haarrandom matrix, this can be done fairly straightforwardly, because each outcome, regardless of collisions, is on average equiprobable [89]. This reduces the problem to a matter of combinatorics, namely of counting the number of desired (no-collision) outcomes versus the number of total outcomes.

Unfortunately, for a circulant matrix, even in the limit where all elements are i.i.d. Gaussian, the outcomes are not on average equiprobable. The reason for this is that adjacent rows have repeated elements (see Fig. 2.5). These repeated elements have two 

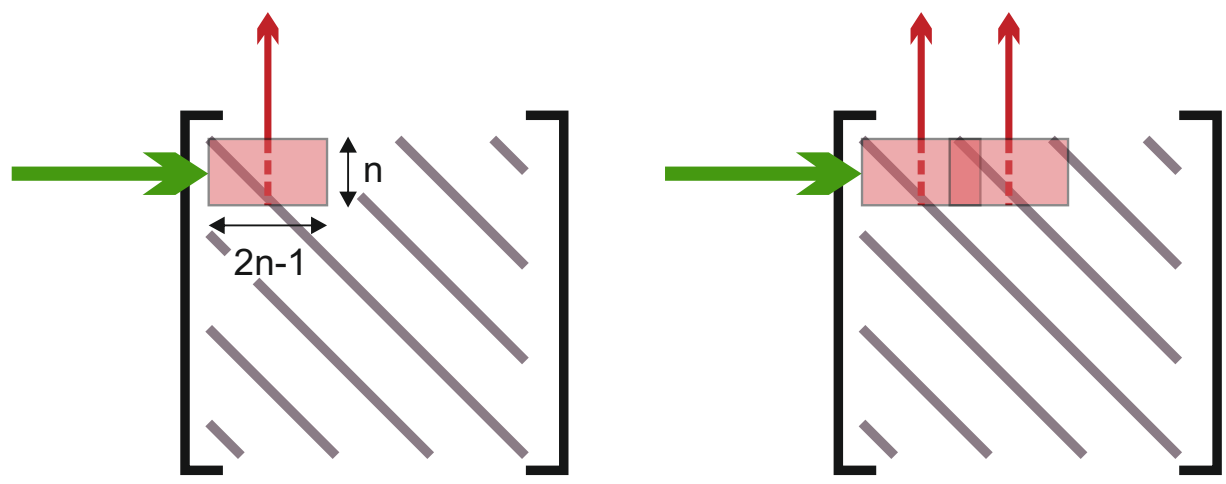

Figure 2.5 Illustration of the counting argument. The input photons (green) enter in the first $n$ modes. If one of these photons comes out of, for example, output mode $n$ (red) then the other photons cannot get out of the first $2 n-1$ output modes. (Left) The red block indicates what output modes are not allowed by 1 photon leaving. (Right) The forbidden regions by two photons may overlap, as long as the used output modes (dashed lines) do not enter the forbidden region of the other photons.

consequences: first, they have the effect that any outcome where two photons are detected $n$ modes apart will have repeated entries in its corresponding matrix $U_{\mathrm{T}}$, meaning that it will certainly not be i.i.d. Secondly, an elementary calculation shows that these repeated elements cause higher-order moments of Gaussians to appear in the calculation, which affect the expected value, making some outcomes more likely than others.

To investigate the scaling of the no-collision condition with the number of photons and the number of modes, we follow the intuition of the previous section and make use of the fact that we have observed that the permanents of submatrices of circulant matrices without repeated elements resemble submatrices of Haar-random matrices in their behavior. We will start with a simple counting argument to find an analytical expression for the fraction of desired outcomes. Then we follow this up with numerical simulations to show that the intuition derived from this argument is correct.

\subsubsection{Number of good outcomes}

The number of desired outcomes, i.e., without circulant correlations, can be found by a counting argument. For now, we assume for simplicity that all outcomes are equiprobable. To show this counting argument, assume without loss of generality that the photons are incident on the first $n$ modes of the network. Each photon blocks exactly $2 n-1$ output modes: on both sides of the output mode the first $n-1$ adjacent modes are not allowed since they contain the same value in the matrix and another +1 because a photon also blocks the its own output mode. See Fig. 2.5 for a schematic representation. These blocked regions may overlap, as long as none of the output modes are in these regions. 
Hence, in this limit, the fraction of good outcomes is given by:

$$
N_{\text {good }}=\frac{m !_{2 n-1} /\left(m-2 n^{2}+n_{2 n-1}\right)}{\left(\begin{array}{c}
m+n-1 \\
n
\end{array}\right)},
$$

where the subscripts denotes the $x^{\text {th }}$ multifactorial, i.e. $n !_{x}=n(n-x)(n-2 x)(n-$ $3 x) \ldots$. Working out these terms and only taking the first two leading terms $\left(n^{k}\right.$ and $\left.n^{k-1}\right)$ in both the numerator and denominator results in:

$$
\begin{aligned}
N_{\text {good }} & =\frac{m(m-2 n+1)(m-4 n+2)(m-6 n+2) \ldots}{(m+n-1)(m+n-2)(m+n-3) \ldots} \\
& \approx \frac{m^{n}-m^{n-1} \sum_{j=0}^{n-1} j(2 n-1)}{m^{n}+m^{n-1} \sum_{j=0}^{n-1}(n-j)} \\
& \approx \frac{m^{n}-\frac{1}{2}(n-1)(n-2)(2 n-1) m^{n-1}}{m^{n}+\frac{1}{2}(n+1) n m^{n-1}} \\
& \approx \frac{m^{n}-n^{3} m^{n-1}}{m^{n}+n^{2} m^{n-1}} .
\end{aligned}
$$

Here the standard series $\sum_{j=0}^{j=n} n=n \frac{n-1}{2}$ has been used. As we are interested in the regime $m \gg n$, we can rewrite it as a Laurent series at $m=\infty$ :

$$
\begin{aligned}
N_{\text {good }} & \approx \frac{1-n^{3} / m}{1+n^{2} / m} \\
& =1-\sum_{j=1}^{\infty}(-1)^{j} \frac{n^{2 j}(n+1)}{m^{j}} \\
& \approx 1-\frac{n^{3}+n^{2}}{m}+\mathcal{O}\left(\frac{n^{5}}{m^{2}}\right) .
\end{aligned}
$$

This shows that the number of modes required to make the number of good (uncorrelated) outcomes dominate the experiment scales as $m \propto n^{3}$. The $n^{3}$ scaling is only a small increase from the normal $m \propto n^{2}$ scaling to hide the collisions. This is remarkable because there are additional correlations present in the circulant matrix and that a circulant matrix only has $n$, not $n^{2}$ unique elements.

\subsubsection{Numerical results}

The above derived scaling for the fraction of possible outcomes without circulant correlations can be verified numerically. Figure 2.6a) shows a Monte-Carlo simulation on how the fraction of correlated outcomes becomes small for some selected number of photons. Each data point is the average of $5 \cdot 10^{6}$ samples, all drawn from independent instances of circulant unitaries. The error bars denote the standard deviation corresponding to a Bernoulli distribution. Each data set has a dashed line indicating the predicted $\frac{1}{n^{3}}$ scaling. 
a)

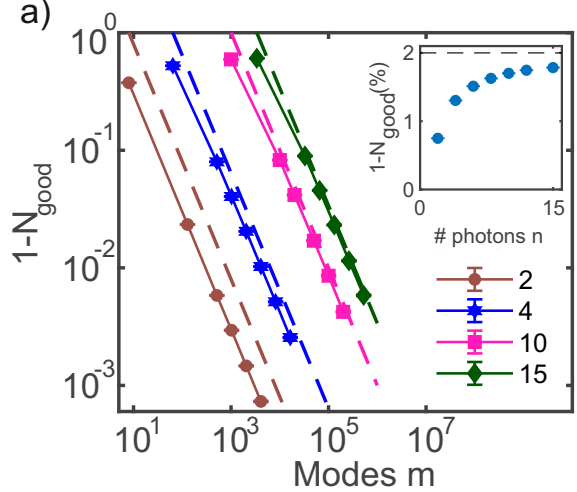

b)

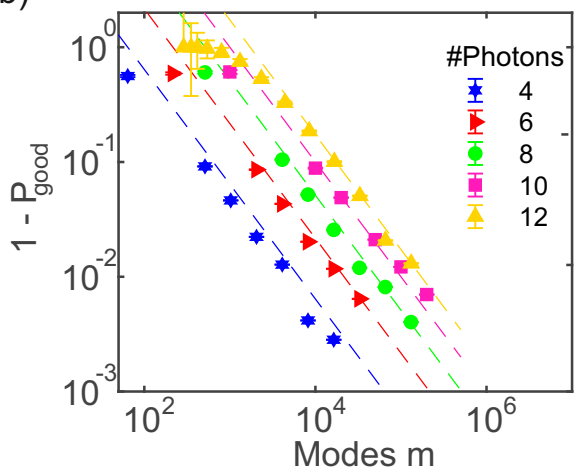

Figure 2.6 Monte-Carlo of no-collisions. a) Monte-Carlo calculation of good outcomes for a selected number of photons. The dashed line is the predicted scaling. Note that the numerical results approach the theory for a large number of photons. Inset: the convergence to the fraction predicted by the combinatorics (dashed line) at $m / n^{3}=50$. b) Monte-Carlo calculation of the number of good outcomes, multiplied with the average probability of a good outcome. The error bars denote the standard deviation of $P_{\text {good }}$. The dashed lines are guides to the eye showing the $\propto n^{3} m^{-1}$ scaling.

The figure hints at a $m \propto n^{3}$ scaling, where the deviation for small photon numbers can be understood as finite size effects and the fact that no uncorrelated output states are possible when $m=n^{2}$. These results confirm the scaling as predicted by the combinatorics of section 2.5.1.

The next step to show no-collisions is to identify the probability of ending up with an uncorrelated outcome and to show that this approaches 1 by increasing the total network size $m$. Therefore we first identify the number of uncorrelated (good) outcomes, just as in Fig. 2.6a, and then multiply that with the average probability associated with these good outcomes.

Figure 2.6b) shows how the probability of obtaining a correlated outcome scales with the number of photons and the number of modes. Each data point is the product of the fraction of good outcomes, similar to Fig. 2.6a), and the corresponding average probability of such outcome. Each sample is drawn from a circulant unitary and hence incorporates the unitary constraint. Note that this is a more stringent scenario than derived above in section 2.5.1. The error bars denote the standard deviation, where again a Bernoulli distribution is used to estimate the uncertainty in $N_{\text {good }}$. For each dataset -number of photons-, there is a dashed line indicating the predicted $m \propto n^{3}$ scaling. 


\subsection{Required hardware}

In this section, we comment on the availability of the hardware needed to implement our proposal.

A key experimental advantage of our proposal is that it removes the need for fibercoupled single-photon sources. For example, in photon sources based on parametric down-conversion, a key source of optical loss is the coupling from the photons into an optical fiber. In our protocol, the photon sources could directly illuminate the scatterer, removing the need for fiber coupling and its associated optical loss.

To implement the random phases, there are three natural candidates: ground glass plates, holographic plates, or spatial light modulators. The first candidate for implementing such random phases, would be a surface scatterer such as a ground glass plate. This naturally implements the phases by random height differences on the surface, which corresponds to a relative phase delay. The second method is to use a holographic plate, made of glass or polymer where the thickness varies throughout the plate. By varying the thickness of the object locally, the light picks up a relative phase depending on the thickness. This method also gives a fixed phase shift, but allows for some degree of design. The transmission of a ground glass plate or a holographic plate is limited by the impedance mismatch between the material used and air, resulting in reflection losses of a few percent of the light. The third method is a fully tunable version of a phase-plate: a phase-only spatial light modulator (SLM). A phase-only SLM typically consists of an LCD screen in which every pixel can give a variable delay to the incident light on that pixel, implementing a phase shift. As SLMs are versatile and induce low losses -up to $90 \%$ transmission efficiency-, SLMs are widely used in scattering adaptive-optics research [76].

Once the photons went through the optical network, they must be detected. For this, a single-photon sensitive camera is needed as the multi-photon interference manifests itself in the transverse plane. Since lumping different output modes together will result in decoherence of the interference pattern, the total number of modes from in the network must be smaller or equal to that of the detector, and each detector pixel should correspond with a diffraction-limited spot. Hence circulant boson sampling requires megapixel singlephoton cameras, which is the major technological limitation of our protocol.

Superconducting nanowire single-photon detectors (SNSPD)s are a promising technology for such detectors. SNSPDs are reported to have record-high detection efficiencies $[96,97]$ for a single detection pixel. Currently SNSPD based cameras are being developed and up to 64 pixels are already reported on a regular basis [98-106]. The first kilopixel detector is already reported as well [107], and current electronics architecture is known to allow up to $225 \times 225$ pixels [108]. The detection efficiency of these devices is limited by the low fill factor on the sensor, typically $\approx 30 \%$ and relative low quantum efficiency, which is typically in the order of $\approx 30-40 \%$. It is estimated that the fill factor, however, can be improved to $\approx 90 \%$ with already existing technology [107]. Furthermore, the detection efficiency can be improved greatly by also placing each of the detection pixels inside an optical cavity. Note that this is the default method to boost detection efficiencies 
close to $100 \%$ in single-pixel SNSPDs [97].

\subsection{Conclusion}

In conclusion, we have described and theoretically analysed a free-space optical method for implementing random matrices suitable for boson sampling in a way that induces only minimal loss while making many optical modes accessible. We anticipate that this method will be useful for high-optical transmission quantum advantage demonstrations in photonics. 



\section{Spontaneous parametric downconversion for boson sampling}

The next milestone in photonic quantum information processing is demonstrating a quantum advantage [10,109], i.e. an experiment in which a quantum optical system outperforms a classical supercomputer. This can be achieved with boson sampling [15]. The aim of boson sampling is, for a given input configuration of photons, to provide a sample of the output configuration from an arbitrary unitary transformation. A photonic quantum device that implements this consists of multiple photon sources, a large passive interferometer and single-photon detectors as shown in Fig. 3.1. This is believed to be easier to implement than a universal quantum computer and resulted in a surge of experiments [17-21, 23, 24, 36, 110]. These experiments require many almost identical photons and practically no losses.

Spontaneous parametric down-conversion (SPDC) sources are a well-known method of generating single photons. A major drawback of building an $n$-photon SPDC source is the probabilistic generation of the photon pairs, meaning that generating $n$ photons simultaneously will take exponentially long. This resulted in doubt regarding the suitability of SPDC for multiphoton experiments as the brightness can only increase at the expense of a decreased purity [33-35]. Scattershot boson sampling improves on this by enabling the generation of $n$ photons in polynomial time using $\sim n^{2}$ sources in parallel [111]. The photons, however, still need to be sufficiently identical.

A way to improve photon indistinguishability is spectral filtering. Unfortunately, this comes at the cost of losses. Losses, too, are detrimental to multiphoton interference experiments as they exponentially increase the experimental runtime [112]. Finding an optimal trade-off between losses and distinguishability is a nontrivial task.

Previous work on optimizing the spectral filtering SPDC sources used restricting assumptions to study the trade-off between spectral purity and symmetric heralding efficiency [113]. Other work on designing SPDC sources has studied optimal focusing parameters for bulk crystal sources and pump beam parameters [114], and phase-matching functions [115]. However, the limitations of SPDC sources for boson sampling, or multiphoton interference in general, have not been studied thus far.

Recently a new classical approximation algorithm for noisy boson sampling was suggested which incorporates both losses and distinguishability [59]. This algorithm gives a lower bound to the amount of imperfections that can be tolerated in order to still achieve a quantum advantage. More importantly, since it incorporates both imperfections, it can be used to trade-off distinguishability and losses. Furthermore, it showed that the best source reported in literature [116] does not meet the standards needed for demonstrating a quantum advantage experiment.

The content of this chapter is adapted from the following published paper: R. van der Meer et al., Phys. Rev. A 101, 063821 (2020). 
In this chapter, we investigate the suitability of SPDC sources for scattershot boson sampling from a complexity theory point of view with a minimal number of approximations. The model of [59] is used to find the optimal source and filter parameters for a boson-sampling experiment. From this we determine a minimal overall transmission efficiency which places a lower bound on the transmission by other experimental components. We target, by convention, a 50-photon boson-sampling experiment [117].

While this is an exploratory study that does not consider all possible crystals, we note that the fact that boson sampling is possible with any SPDC source is of interest. Therefore, we limit ourselves to a few promising crystals since these are the most likely candidates to demonstrate a suitable boson-sampling source. The considered SPDC crystals are: potassium titanyl phosphate (ppKTP), fi-barium borate (BBO) and potassium dihydrogen phosphate (KDP). KTP is a popular choice since it has symmetric group velocity matching at telecom wavelengths $[30,118]$, which is favorable for obtaining pure states. The photon generation rates of KTP sources are high as it uses periodic poling. Moreover, periodic poling allows Gaussian-shaped phase-matching functions by means of Gaussian apodization [119-123]. The second crystal, BBO, is known for generating the current record number of photons $[29,36]$ and also generates photons at telecom wavelength. However, it has asymmetric group velocity matching, resulting in a reduced spectral purity. Finally, the last crystal we consider is KDP. KDP sources, which generate photons at $830 \mathrm{~nm}$, are known to generate one of the highest purity photons without filtering [124].

Our calculations consider Gaussian-shaped pulses to pump the SPDC process in a collinear configuration. We assume the existence of only one spatial mode and do not take into account focusing effects. This is a valid assumption for both waveguide sources as well as for bulk sources without focusing. Focusing increases the number of spatial modes and hence affects the spectral purity [114]. Furthermore, higher-photon-number states are ignored, which is reasonable given the existence of photon-number-resolving detectors [125]. The effect of multi-photon contributions are explored in Appendix 3.A.

\subsection{Theory}

\subsubsection{SPDC sources}

SPDC sources turn a pump photon into two down-converted photons, and hence produce photons in pairs. For Type-II SPDC, the two photons from the pair each emerge in a separate mode. Traditionally these modes are referred to as signal and idler. The SPDC process can be understood by considering energy conservation $\hbar \omega_{\mathrm{p}}=\hbar \omega_{\mathrm{s}}+\hbar \omega_{\mathrm{i}}$ as well as momentum conservation $\overrightarrow{k_{\mathrm{p}}}=\overrightarrow{k_{\mathrm{s}}}+\overrightarrow{k_{\mathrm{i}}}$, where $\mathrm{p}$, s and i denote the pump, signal and idler photons, respectively. Momentum conservation can be tweaked by quasi phase matching by either periodic or apodized poling. Both energy and momentum conservation only allow certain wavelength combinations and together they specify the spectral-temporal properties of the two-photon state [126].

Birefringence results in an asymmetry between the signal and idler photon. This 


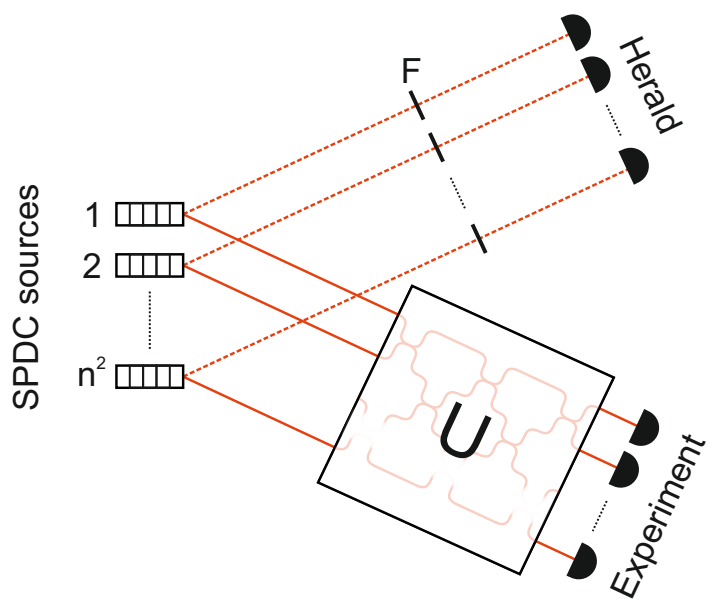

Figure 3.1 Scattershot boson sampling. A $n$-photon scattershot boson-sampling experiment has $n^{2}$ heralded single-photon sources. Each source can send a photon to one of the input modes of the interferometer $U$. The other photon (dashed) is filtered (F) and is used as a herald.

leads to spectral-temporal correlations between the two. Such correlations reduce the spectral purity $P_{\mathrm{x}}=\operatorname{Tr}\left(\rho_{\mathrm{x}}^{2}\right)$, where $\rho_{\mathrm{x}}$ is the reduced density matrix of photon $\mathrm{x}$. When no correlations exist, the photon state is factorizable and the photons are spectrally pure [127].

A visual representation of the two-photon state is shown in Fig. 3.2. The spot in the center indicates the two-photon state with what probability the photons are in this region of the frequency space. This probability is also referred to as the joint spectral intensity (JSI), which is related to the joint spectral amplitude (JSA) by JSI $=|\mathrm{JSA}|^{2}$. The JSA describes the wavefunction of the photon pair as a function of the wavelength of the photons and follows from energy and momentum conservation. The factorizability of the JSA determines the spectral purity of the source.

We now proceed with a mathematical description of SPDC and subsequently the JSA. The interaction hamiltonian for a collinear Type-II SPDC is given by [29]

$$
H_{\mathrm{I}}=i \hbar \eta a_{\mathrm{s}}^{\dagger} a_{\mathrm{i}}^{\dagger}+\text { h.c., }
$$

where the conversion efficiency is given by $\eta \propto \chi^{(2)} E_{\mathrm{p}}$, with $a_{\mathrm{x}}^{\dagger}$ the creation operator on mode $\mathrm{x}, \chi^{(2)}$ the crystals second-order nonlinear susceptibility and $E_{\mathrm{p}}$ the amplitude of the pump field. From this interaction hamiltonian, the time evolution of the SPDC state generation can be computed [128]

$$
|\psi(t)\rangle=\operatorname{sech}(\mu) \sum_{n=0}^{\infty} \tanh ^{n} \mu\left|n_{\mathrm{s}}, n_{\mathrm{i}}\right\rangle,
$$

where the signal and idler modes are assumed to initially be in the vacuum state and with 
a)

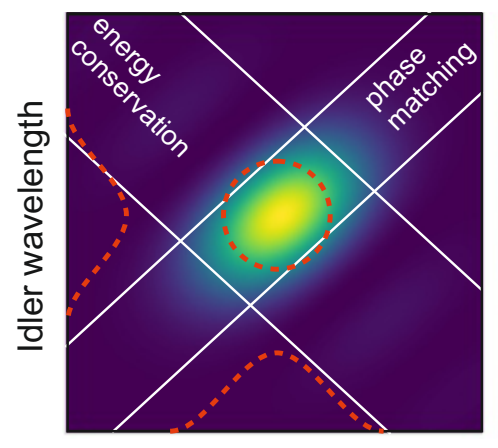

Signal wavelength b)
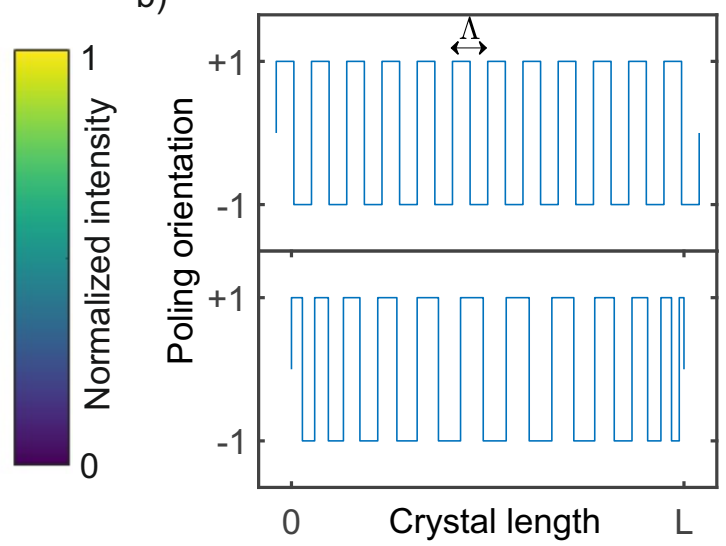

Figure 3.2 Joint Spectral Intensity. a) An example of a joint spectral intensity (JSI). The red dashed line shows the Gaussian filter for both the signal and idler photon. The (anti)diagonal white lines denote the region which satisfies phase matching (energy conservation). b) Sketch of two different types of periodic poling. Top: standard periodic poling. Bottom: Gaussian apodized aperiodic poling.

$\mu=\eta t$. In the limit of $\mu \ll 1$, this can be approximated to

$$
|\psi\rangle \approx \sqrt{1-\mu^{2}} \sum_{n=0}^{\infty} \mu^{n}|n, n\rangle .
$$

This quantum state has an explicit frequency dependence which follows directly from energy and momentum conservation. The energy conservation $\alpha\left(\omega_{\mathrm{s}}, \omega_{\mathrm{i}}\right)$ function is a Gaussian pulse with a center wavelength $\omega_{\mathrm{p}}$ and bandwidth $\sigma_{\mathrm{p}}$ :

$$
\alpha\left(\omega_{\mathrm{s}}, \omega_{\mathrm{i}}\right)=\exp \left(\frac{-\left(\omega_{\mathrm{s}}+\omega_{\mathrm{i}}-\omega_{\mathrm{p}}\right)^{2}}{4 \sigma_{\mathrm{p}}^{2}}\right) .
$$

The phase-matching function for a periodically poled crystal is given by:

$$
\phi\left(\omega_{\mathrm{s}}, \omega_{\mathrm{i}}\right)=\operatorname{sinc}\left(\frac{k_{\mathrm{p}}-k_{\mathrm{s}}-k_{\mathrm{i}}-\frac{2 \pi}{\Lambda}}{2} L\right),
$$

with $L$ the length of the nonlinear crystal and $\Lambda$ the poling period. The sinc function arises from a Fourier transform of the second-order nonlinearity, which is a rectangular function in space. Another type of quasi phase matching exists, which is Gaussian apodization. An example of both a standard periodic and a Gaussian aperiodic poling is shown in Fig. $3.2 \mathrm{~b}$ ). By varying the poling domain size, the side lobes of each sinc average out. This leads to a phase-matching function: [119]

$$
\phi_{\mathrm{G}}\left(\omega_{\mathrm{s}}, \omega_{\mathrm{i}}\right)=\exp \left(-\frac{\gamma \Delta k^{2} L^{2}}{4}\right),
$$


where $\gamma \approx 0.193$, such that the width of this phase-matching function equals that of Eq. 3.5. The parameter $\Delta k$ denotes the phase mismatch and $L$ again the crystal length. The energy conservation function together with the appropriate phase-matching function give the JSA:

$$
f\left(\omega_{\mathrm{s}}, \omega_{\mathrm{i}}\right)=\alpha\left(\omega_{\mathrm{s}}, \omega_{\mathrm{i}}\right) \phi\left(\omega_{\mathrm{s}}, \omega_{\mathrm{i}}\right)
$$

The two-photon state corresponding to this JSA can give rise to distinguishability. This can be mitigated by spectral filtering. The overall two-photon state after filtering can now be written as:

$$
|\psi\rangle=\iint d \omega_{\mathrm{s}} d \omega_{\mathrm{i}} f\left(\omega_{\mathrm{s}}, \omega_{\mathrm{i}}\right) F_{\mathrm{s}, i}\left(\omega_{\mathrm{s}}, \omega_{\mathrm{i}}\right)\left|1_{\mathrm{s}}\right\rangle\left|1_{\mathrm{i}}\right\rangle,
$$

where $F_{\mathrm{s}, i}\left(\omega_{\mathrm{s}}, \omega_{\mathrm{i}}\right)$ denotes a possible filter function on the signal and/or idler photon. For simplicity, we ignore the vacuum and multiphoton states.

The spectral purity of the photon pair can be found with a Schmidt decomposition of the JSA $[129,130]$. From this follows a Schmidt number $K$ which determines the spectral purity

$$
P=\frac{1}{K}
$$

Physically, $K$ is the effective number of modes that is required to describe the JSA (e.g., see [131]). When $K=1$ the photon pair is factorizable. In this case, detecting a photon as herald leaves the other photon in a pure state. In Fig. 3.2 this would manifest itself such that the JSA becomes aligned with the axes. In case $K>1$, detecting one photon leaves the other photon in a mixed state of several modes. Hence, the remaining photon has a lower spectral purity.

It is possible to improve the spectral purity by filtering the photons. The effect of filtering can be understood as overlaying the filter function over the JSA. This is shown with the dashed lines in Fig. 3.2. A well-chosen filter removes the frequency correlations between the photons, but inevitably introduces losses, which in turn are detrimental for boson-sampling experiments.

\subsubsection{Classical simulation of boson sampling with imperfections}

The presence of imperfections such as losses [67] and distinguishability [66] of photons reduces the computational complexity of boson sampling. Classical simulation algorithms of boson sampling upper bound the allowed imperfections. These classical simulations approximate the boson sampler outcome with a given error.

We now present the model of [59]. This model approximates an imperfect $n$-photon boson sampler where $n-m$ photons are lost, by describing the output as up to $k$-photon quantum interference $(0 \leq k \leq m)$ and at least $m-k$ classical boson interference. Furthermore, this formalism naturally combines losses and distinguishability into a single simulation strategy, thereby introducing an explicit trade-off between the two. In this 
model, the error bound $E$ of the classical approximation is given by

$$
E<\sqrt{\frac{\alpha^{k+1}}{1-\alpha}} .
$$

The parameter $\alpha$ which we will refer to as the 'source quality' is given by

$$
\alpha=\eta x^{2}
$$

with $\eta=m / n$ denotes the transmission efficiency per photon. Losses in different components are equivalent, so different losses can be combined into a single parameter $\eta$ [132]. The average overlap of the internal part of the wave function between two photons is given by $x=\left\langle\psi_{\mathrm{i}} \mid \psi_{\mathrm{j}}\right\rangle(\mathrm{i} \neq \mathrm{j})$. Therefore $x^{2}$ is the visibility of a signal-signal Hong-Ou-Mandel interference dip [133]. This indistinguishability equals the spectral purity.

This model allows for optimizing the SPDC configuration by optimizing the source quality of Eq. 3.11, which effectively trades-off the losses and distinguishability. Furthermore, from Eq. 3.10 the maximal number of photons $k$ can be calculated by specifying a desired error bound.

\subsection{Methods}

In order to find the best SPDC configuration for a selection of crystals, we run an optimization over the SPDC settings to maximize $\alpha$ while varying the filter bandwidth. Since we consider collinear SPDC, the optimization parameters are the crystal length $L$ and the pump bandwidth $\sigma_{\mathrm{p}}$. Note that these parameters determine the shape of the JSA and therefore the separability. The pump center wavelength is set such that group velocity dispersion is matched [124, 134-136]. From our numerical calculations we observe that the optimization problem appears to be convex over the region of the parameter space of interest. We note that the optimization parameters are bounded, e.g., the crystal length cannot be negative. A local optimization routine (L-BFGS-B, Python) was used.

The source quality $\alpha$ can be calculated from the JSA. The JSA was calculated numerically by discretizing the wavelength range of interest (see Appendix B). The wavelength range was chosen to include possible side lobes of the sinc phase-matching function. The spectral purity is calculated from the discretized JSA using a singular value decomposition (SVD) [137]. The transmission efficiency is calculated by the overlap of the filtered and unfiltered JSA. In other words, only 'intrinsic' losses are considered and experimental limitations such as additional absorption by optical components or absorption losses in the crystal are not taken into account. This is permissible since such experimental losses are constant over the wavelength range.

The introduction of wavelength-independent losses does not change the position of the optimum, as it only reduces the transmission efficiency. Wavelength-dependent losses can be understood as an additional filter. 
a)

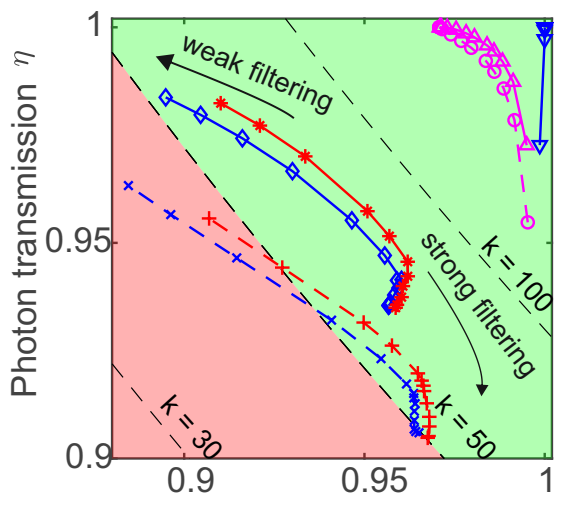

Photon indistinguishability $\mathrm{x}^{2}$ b)

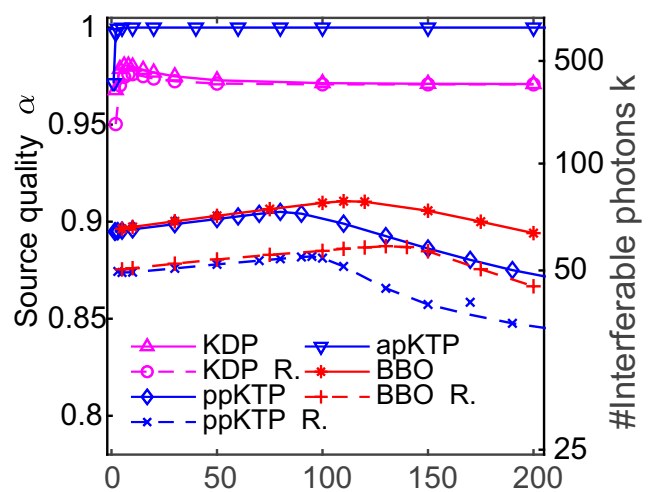

Filter bandwidth FWHM (nm)

Figure 3.3 Upperlimit of SPDC sources. a) The transmission efficiency per photon $\eta$ and indistinguishability $x^{2}$ corresponding to the ideal SPDC settings at different filter bandwidths for different crystals (see legend in $\mathrm{b}$ ). The dashed lines are isolines, indicating how many photons $k$ can be used for a boson-sampling experiment. The lines between the points are guides to the eye. The indistinguishability and transmission efficiency together result in the source quality factor $\alpha=x^{2} \eta$. b) The values of $\alpha$ and the corresponding number of photons $k$ (right axis) as functions of the filter bandwidth. In the legend $\mathrm{R}$. denotes a rectangular filter, otherwise a Gaussian filter was used.

Realistic SPDC settings are guaranteed by constraining the crystal length and pump bandwidth values in the optimizer. The crystal lengths are bounded by what is currently commercially available. The pump bandwidth is bounded to a maximum of roughly $25 \mathrm{fs}(\Delta f \approx 17 \mathrm{THz})$ pulses. Such pulses can be realized with commercial Ti:Sapphire oscillators. See appendix 3.C for the exact bounds and further details. Furthermore, we consider Gaussian-shaped and rectangular-shaped bandpass filters. Rectangular filters are a reasonable approximation of broadband bandpass filters.

In the calculations, only the herald photon is filtered. Also filtering the other photon reduces the heralding efficiency. Typically the increase in purity is not worth the additional losses, especially if finite transmission efficiency of filters are included.

\subsection{Results}

We now proceed by using the metric of [59] to compute the optimal filter bandwidth, pump bandwidth and crystal length for KTP, BBO and KDP sources. The upper bound for the error of the classical approximation (Eq. 3.10) is set on the conventional $E=0.1$.

Figure 3.3a) is a parametric plot of the source quality $\alpha$. The transmission efficiency $\eta$ is shown on the $y$-axis and signal-signal photon indistinguishability $x^{2}$ on the $\mathrm{x}$-axis. The ideal boson-sampling experiment is located at the top right. Each point represents 
an optimal SPDC configuration that maximizes $\alpha$ for that crystal corresponding to a fixed filter bandwidth. The black dashed isolines indicate the maximum number of photons $k$ one can interfere, i.e., they are solutions of Eq. 3.10 for a fixed $E$ and $\alpha$. The weak-filtering regime is in the top left, and the strong-filtering regime is in the bottom right.

Figure 3.3b) represents the source quality $\alpha$ from Fig. 3.3a) explicitly as a function of the filter bandwidth. The left y-axis indicates the source quality $\alpha$. The right y-axis shows the corresponding maximal number of photons $k$. Both graphs show that there is a filter bandwidth that maximizes $\alpha$. From this maximal $\alpha_{\text {opt }}$ the minimal transmission budget $\eta_{\mathrm{T} B}$ can be defined

$$
\eta_{\mathrm{TB}} \alpha_{\mathrm{opt}}=\alpha_{50}
$$

where $\alpha_{50}$ denotes the required value of $\alpha$ to perform a 50-photon boson-sampling experiment. The transmission budget defines the minimum required transmission efficiency for all other components together. This includes, for instance, non-unity detector efficiencies. The maximal $\alpha_{\text {opt }}$ for each crystal and the corresponding SPDC settings are shown in table 3.1 .

The physical intuition behind the curves in Fig. 3.3a) is the following. In case of weak to no filtering (top left in Fig. 3.3a)), the transmission efficiency is the highest and the spectral purity the lowest. In this weak filtering regime, the crystal length and pump bandwidth are such that the JSA is as factorizable as it can be without filtering. This can also be seen in Fig 3.4. Examples of such JSAs can be found in Appendix A.

If we now increase filtering, we arrive at the regime of moderate filtering, at the center of Fig. 3.3a). While increasing the filtering, the optimal crystal length increases and the optimal pump bandwidth decreases. This results in a relative increase of the transmission efficiency, since the unfiltered JSA is now smaller and 'fits easier' in the filter bandwidth. This is the regime with the optimal value for $\alpha$.

In the case of stronger filtering, the losses start to dominate. The optimal strategy in this regime is to make the JSA as small as possible, such that as much of the photons can get through. By doing so, the 'intrinsic' purity, i.e., before filtering, reduces since this configuration does no longer result in a factorizable state. However, this reduction of purity is compensated by the spectral filter. This is shown in Fig. 3.4, where the 'intrinsic' purity decreases, but the filtered purity increases.

Furthermore, this physical picture also explains the differences between a rectangular and Gaussian filter window. The first difference is that a Gaussian filter allows for higher values of $\alpha$ and thus for more photons in a boson-sampling experiment. The second difference is the optimal filter bandwidth. Both differences can be explained by noting that a rectangular filter window ideally only filters out the side lobes. As a result, it cannot increase the factorability of the 'main' JSA, i.e., the part without the side lobes.

For the apodized sources, filtering does not significantly improve the spectral purity. This is because the Gaussian phase-matching function does not introduce side lobes in the JSA and therefore limits possible spectral correlations. The remaining source for such correlations is group-velocity dispersion, which is small around $1582 \mathrm{~nm}$ [30]. 


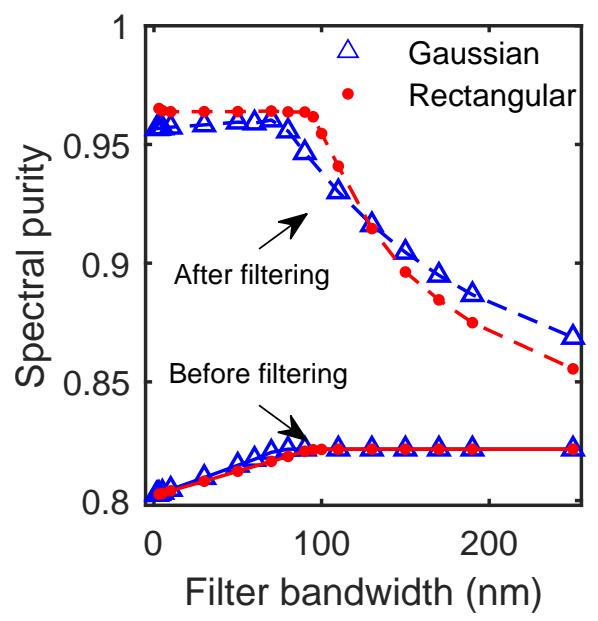

Figure 3.4 The spectral purities of a ppKTP source with a sinc phase-matching function. Every point corresponds to the optimal configuration for this source with such a filter. The purity of these sources is shown before (solid) and after (dashed) filtering. The lines are guides to the eye.

\subsection{Discussion}

It is well known that the spectral purity of symmetrically group-velocity-matched SPDC sources is invariant to changes of either the crystal length or pump bandwidth, as long as the other one is changed accordingly. However, Fig. 3.4 shows that relation no longer holds when filtering is included. In the regime of strong filtering, $\alpha$ is dominated by the losses. Therefore, the SPDC configuration which optimizes $\alpha$ inevitably is the one that minimizes the losses. Hence the spectral purity reduces, but this is compensated by the strong filtering.

In an experiment, the non-unity transmission efficiency of a filter at the maximum of the transmission window will be an important source of losses. As a consequence, spectral filtering is only useful when the filter's maximum transmission is larger than $\alpha_{\mathrm{f}} / \alpha_{0}$, where $\alpha_{\mathrm{f}}$ denotes the filtered $\alpha$ and $\alpha_{0}$ the unfiltered case. If the filter's transmission is lower, then the gain in $\alpha$ is not worth the additional losses.

We note that the ideal filter bandwidths of Tab. 3.1 are larger than what is reported in [113]. We attribute this difference to two points. Firstly, the model of [113] approximates the sinc phase-matching function as a Gaussian. This eliminates the side lobes and hence reduces the losses and it overestimates the spectral purity. As a consequence, smaller filter bandwidths are optimal. Secondly, the model of [113] focuses on the symmetrized heralding efficiency where both photons are filtered. 
Table 3.1 The values of $\alpha_{\text {opt }}$ and the loss budget for a $k=50$ photon boson-sampling experiment for different crystals at a center wavelength $\lambda_{\mathrm{c}}$. The corresponding SPDC settings (crystal length $L$, pump bandwidth $\sigma_{\mathrm{p}}$ and filter bandwidth $\sigma_{\mathrm{f}}$ ) are also listed. The mentioned bandwidths are FWHM of the fields.

\begin{tabular}{l|cccccc}
\hline \hline Crystal & $\alpha_{\text {opt }}$ & $\eta_{\mathrm{TB}}$ & $\begin{array}{c}\lambda_{\mathrm{c}} \\
(\mathrm{nm})\end{array}$ & $\begin{array}{c}L \\
(\mathrm{~mm})\end{array}$ & $\begin{array}{c}\sigma_{\mathrm{p}} \\
(\mathrm{nm})\end{array}$ & $\begin{array}{c}\sigma_{\mathrm{f}} \\
(\mathrm{nm})\end{array}$ \\
\hline KDP & 0.980 & 0.892 & 830 & 25 & 2.3 & 6 \\
KDP R. & 0.976 & 0.896 & 830 & 25 & 2.4 & 10 \\
ppKTP & 0.905 & 0.967 & 1582 & 0.5 & 21.34 & 80 \\
ppKTP R. & 0.882 & 0.992 & 1582 & 0.5 & 20.97 & 95 \\
apKTP & 0.999 & 0.875 & 1582 & 30 & 0.40 & $>10$ \\
BBO & 0.911 & 0.961 & 1514 & 0.95 & 30 & 110 \\
BBO R. & 0.887 & 0.986 & 1514 & 0.94 & 30 & 130 \\
\hline \hline
\end{tabular}

R.: Rectangular filter window

\subsection{Conclusion}

In conclusion, we have numerically optimized SPDC sources for scattershot boson sampling. Using the recently found source quality parameter $\alpha$ [59] we have investigated the fundamental limits of popular SPDC sources for multiphoton experiments.

From the results of Tab. 3.1 we conclude that SPDC sources allow the demonstration of a quantum advantage with boson sampling. The most suitable source for boson sampling is an apKTP crystal. Such a source can have a maximal source quality $\alpha_{\text {opt }}=0.99$ and has a corresponding transmission budget of $87 \%$. This transmission budget is sufficient to incorporate state-of-the-art[138-140] detector efficiencies and keep a small buffer for additional optical losses.

The other two sources are asymmetrically group-velocity-matched sources. The KDP source with a maximal source quality of $\alpha_{\mathrm{opt}}=0.98$ is a good alternative. The optimal source quality for BBO is found to be comparable with ppKTP and less suited for a boson sampling experiment. The fact that these asymmetrically group-velocity-matched sources perform less than symmetrically matches sources is consistent with previous findings.

The limited tolerance for additional losses for the Gaussian apodized KTP source suggests that both waveguide sources and bulk sources without focusing of the pump beam are ideal. Such sources have a single spatial mode and thus do not suffer from an additional reduction of distinguishability which is inevitable with focusing [114].

This work can be extended to other SPDC sources such as [141-143], four-wave mixing sources [39] and to Gaussian boson sampling [32, 65]. The latter can be realized by including the distinguishability between the signal and idler photons. 


\section{ApPEndix 3}

\section{A Effect of multiphoton states on the purity}

The main text assumes that the effect of multiphoton states are negligible to the state purity for typical SPDC sources. In this appendix, we explore the validity of this claim by deriving how the state purity decreases when these multiphoton states are included. We start with the general two-mode state of SPDC, where we omit labels indicating the signal and idler photon

$$
|\psi\rangle=\sqrt{1-\mu^{2}} \sum_{n=0}^{\infty} \mu^{n}|n, n\rangle,
$$

and then compute the density operator

$$
\begin{aligned}
\rho & =|\psi\rangle\langle\psi| \\
& =\left(1-\mu^{2}\right) \sum_{n, m=0}^{\infty} \mu^{\mathrm{n}+m}|n, n\rangle\langle m, m| \\
\rho^{2} & =\left(1-\mu^{2}\right)^{2} \sum_{n, m=0}^{\infty} \mu^{2(n+m)}|n, n\rangle\langle m, m| .
\end{aligned}
$$

It is easy to compute the purity from the expression for $\rho^{2}$

$$
\begin{aligned}
\operatorname{Tr}\left(\rho^{2}\right) & =\sum_{x=0}^{\infty}\left\langle x, x\left|\left(\rho^{2}\right)\right| x, x\right\rangle \\
& =\left(1-\mu^{2}\right)^{2} \sum_{x=0}^{\infty}\left\langle x, x\left|\left(\mu^{4 x}|x, x\rangle\langle x, x|\right)\right| x, x\right\rangle \\
& =\left(1-\mu^{2}\right)^{2} \sum_{x=0}^{\infty} \mu^{4 x}
\end{aligned}
$$

where $x$ is a variable to compute the trace and $\mu$ is assumed to be real. This equation can be simplified further by using the geometrical series $(|\mu| \approx 0.01 \ll 1)$

$$
\operatorname{Tr}\left(\rho^{2}\right)=\left(1-\mu^{2}\right)^{2} \frac{1}{1-\mu^{4}}
$$

Figure 3.5 offers a graphical representation of Eq. 3.16. It shows that the contribution of multiphoton states in SPDC only reduces the purity slightly in the typical experimental generation probabilities. A typical experimental value of $\mu=0.01$ results in a purity of 0.998, which confirms the statement in the main text that the contribution of multiphoton states on the state purity is indeed negligible. 


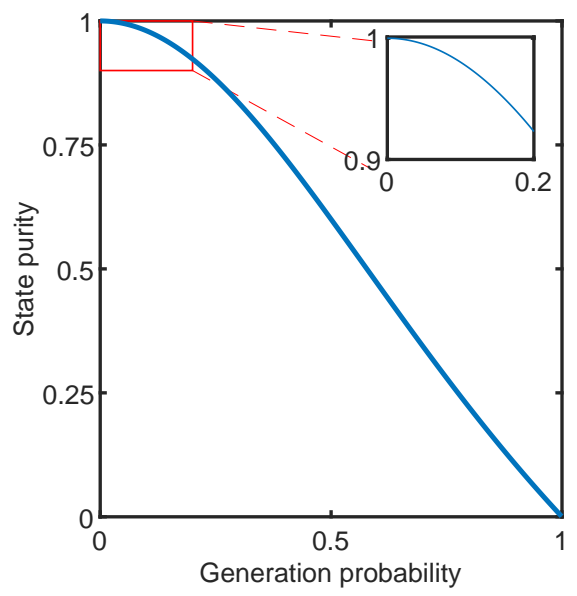

Figure 3.5 Purity with multiphoton states. The reduction of state purity as a function of the generation probability $\mu$. The inset shows a zoom-in in the typical operational region of SPDC sources.

\section{B Optimal SPDC settings}

The effect of the filter bandwidth on the optimal SPDC configuration (except for apKTP) can be categorized in three different regimes. These regimes are the weak, moderate and strong filtering regime. An example of the JSA of a ppKTP source in all three regimes can be seen in Fig. 3.7.

The corresponding SPDC configuration parameters can be seen in Fig. 3.6. This figure shows that in the weak filtering regime, the bounds on the crystal sizes and pump bandwidth can be reached. Once such a bound is reached, the SPDC configuration loses a parameter to optimize the JSA factorizability with, meaning that the general trend of matching the crystal length and pump bandwidth cannot continue anymore. This limits the purity. In case of ppKTP, the limiting factor is the crystal length, whereas in case of a $\mathrm{BBO}$ source the maximum pump bandwidth is the limiting factor.

\section{C Numerical stability}

We used a local optimization algorithm to find the optimal SPDC configuration for different filter bandwidths. Each iteration of this algorithm computes the spectral purity and losses by discretizing the (filtered) JSA. Such a numerical approach can fail and/or give wrong results. The algorithm can fail because the problem is not convex or that it finds unphysical results (such as a negative crystal length). The algorithm can give wrong results if the discretization of the JSA is too coarse.

By bounding the parameter space we guarantee that the algorithm does not reach 

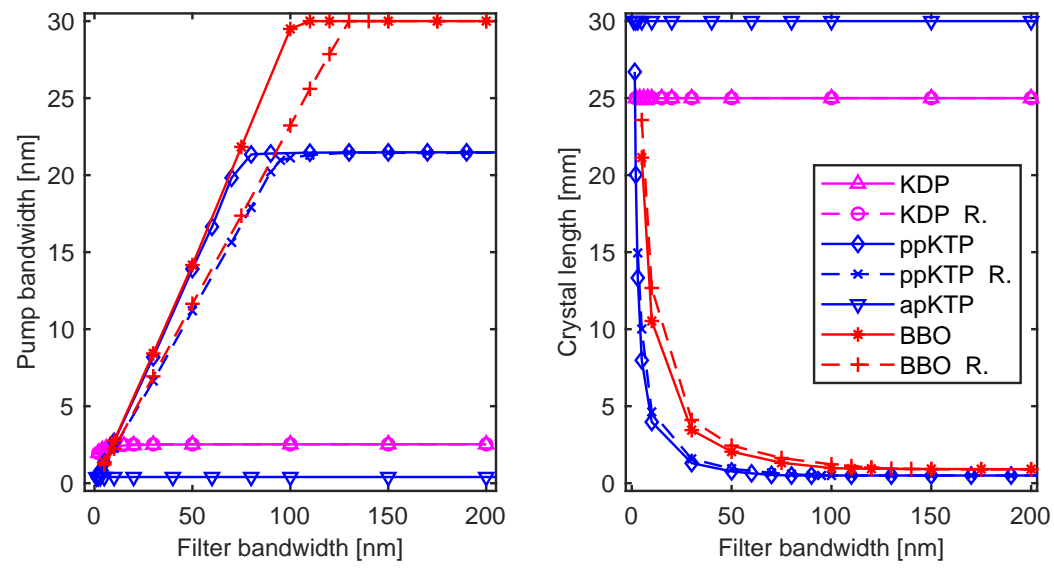

Figure 3.6 Optimal source parameters. The optimal pump bandwidth and crystal length as a function of the filter bandwidth.

unphysical results. Furthermore, we note that optimizing over the whole parameter space, i.e., the filter bandwidths, crystal lengths and pump bandwidths is not a convex problem. This problem is solved by optimizing the crystal and pump properties each time for different filter bandwidths.

The discretization of the JSA can cause numerical errors. Increasing the number of grid points, i.e., increasing the resolution, decreases this numerical error. Increasing the resolution results to a convergence of the result. Unfortunately, it is not directly known how our numerical calculation converges to a reliable answer. How to a priori estimate the numerical error for a given discretization is also unclear.

In order to show that our calculations have converged, we simply try different discretizations of the JSA. For every discretization, we calculate the corresponding source quality $\alpha$ and observe how it is varies. Figure 3.8 shows that the numerical error originating from this discretization is small in the limit of more than $2000^{2}$ (2000 per photon) grid points. This confirms the validity of our calculations. Table 3.2 provides an overview of all relevant parameters for the stability of the simulation. 


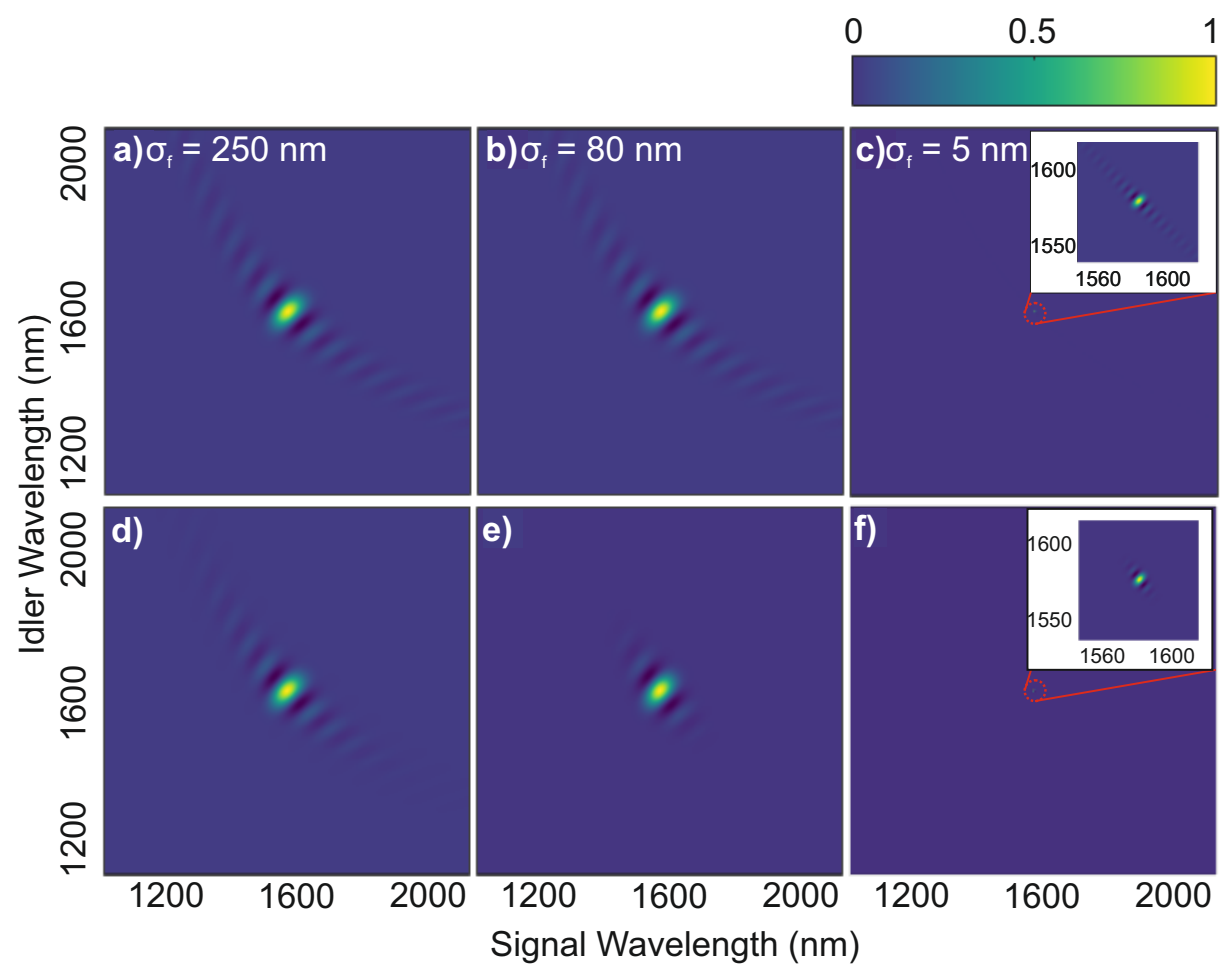

Figure 3.7 Example JSI. The real part of the JSA of the optimal ppKTP SPDC configuration in case of weak filtering with (left), optimal filtering (center) and strong filtering (right). The top panels show the JSA before filtering, the bottom panels after filtering.

Table 3.2 The simulation parameters for each crystal. The bounds on the crystal lengths and pump bandwidth are given, just as the range of wavelength over which the JSA is computed. The grid points are the number of steps used to discretize the entire wavelength range

\begin{tabular}{|c|c|c|c|c|c|c|c|c|}
\hline \multirow[t]{2}{*}{ Crystal } & \multicolumn{2}{|c|}{$\begin{array}{c}\text { Crystal } \\
\text { length }(\mathrm{mm})\end{array}$} & \multicolumn{2}{|c|}{$\begin{array}{c}\text { Pump } \\
\text { bandwidth }(\mathrm{nm})\end{array}$} & \multicolumn{2}{|c|}{$\begin{array}{l}\text { Wavelength } \\
(\mathrm{nm})\end{array}$} & \multirow[t]{2}{*}{$\begin{array}{l}\text { Grid } \\
\text { points }\end{array}$} & \multirow[t]{2}{*}{$\begin{array}{l}\text { Sellmeier } \\
\text { constants }\end{array}$} \\
\hline & $\min$. & $\max$. & $\min$. & $\max$. & $\min$. & $\max$. & & \\
\hline KTP & 0.5 & 30 & 0.1 & 30 & 1028 & 2136 & $2000^{2}$ & {$[144,145]$} \\
\hline $\mathrm{BBO}$ & 0.5 & 40 & 0.1 & 30 & 1008 & 2093 & $2000^{2}$ & [146] \\
\hline KDP & 0.5 & 25 & 0.1 & 10 & 780 & 880 & $1500^{2}$ & [147] \\
\hline
\end{tabular}




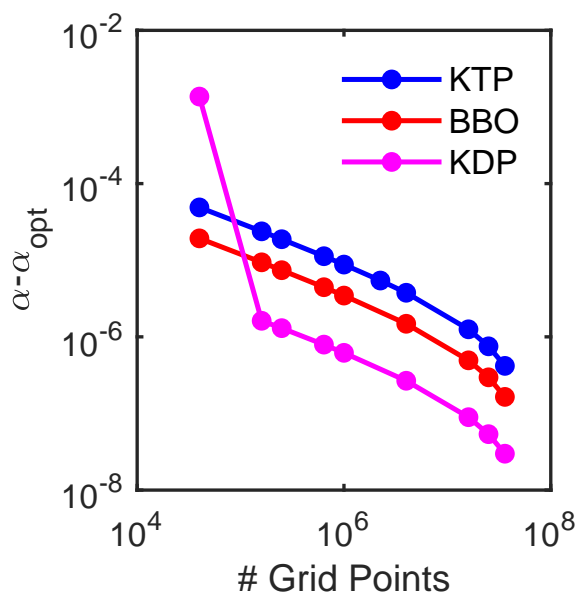

Figure 3.8 Discretization error. The convergence of the source quality $\alpha$ with the discretization of the frequency space. Each data point is the difference of $\alpha$ with the $\alpha$ corresponding to $8000^{2}$ grid points. All crystals, ppKTP, BBO and KDP, are set in their optimal configuration. 



\section{Multiphoton setup}

The single photons used in the multiphoton experiments in this thesis are generated by a multiphoton source. This multiphoton source should generate photons at telecom wavelengths, in our case $1550 \mathrm{~nm}$ since all experiments in this thesis are performed on an integrated quantum photonic chip that is optimized for this wavelength. We also refer to this chip as an optical or photonic processor. Simply having photons at telecom wavelengths is not sufficient, as quantum interference requires the generated photons to be indistinguishable. Furthermore, the multiphoton source should generate these photons at sufficiently high rates to guarantee feasible experimental run times. Simultaneously, the contribution of higher photon states should be small. As shown in Chapter 3, spontaneous parametric downconversion (SPDC) based on periodically poled KTP crystals makes a suitable source for this type of experiments.

This chapter will give a detailed description of the multiphoton setup used for all experiments described in this thesis. To this end, the multiphoton source and the associated hardware will be described in some detail and the properties of the generated photons will be discussed. Finally, the chapter concludes with an outlook, in which possible improvements to the setup are explored.

\subsection{Setup}

A schematic of the multiphoton setup is shown in Fig. 4.1. The setup consists out of two distinct parts. Firstly, there is the laser and some associated diagnostics to monitor the laser's performance. Secondly, there are nonlinear crystals to generate pairs of photons. This part consists out of two identical single-photon sources, each probabilistically producing pairs of photons using SPDC. The source feeds into the optical processor on which the various experiments are carried out. The second part of the setup consists of the detectors and the time tagger. This last part is not shown in Fig. 4.1, as their exact configuration depends on the particular experiment that is performed.

\subsubsection{Multiphoton source}

The photon source is pumped by a 150 femtosecond mode-locked Ti:Sapphire oscillator (Tsunami, Spectra Physics), with a central wavelength of $775 \mathrm{~nm}$, a linewidth of about $5.5 \mathrm{~nm}$ and a pulse repetition rate of $80 \mathrm{MHz}$. The average output power is almost $1 \mathrm{~W}$ and can be manually reduced further by the half-wave plate and polarizing beam splitter immediately after the oscillator. A fraction of the pump power is tapped off by a beam sampler (BS4) to monitor the laser's performance with a calibrated photodiode (PD2: PDA36A, Thorlabs) and a spectrometer (SP: USB 4000, Ocean Optics). Furthermore, another photodiode (PD1: TDA 200, PicoQuant) is used to generate a trigger signal, which can be used later to signal the time window in which photons are expected to arrive at the detectors.

Before the pump light arrives at the nonlinear crystals, its beam size is expanded by a factor 2 to about $3 \mathrm{~mm}$. This is required as focus parameters at the crystals are important 


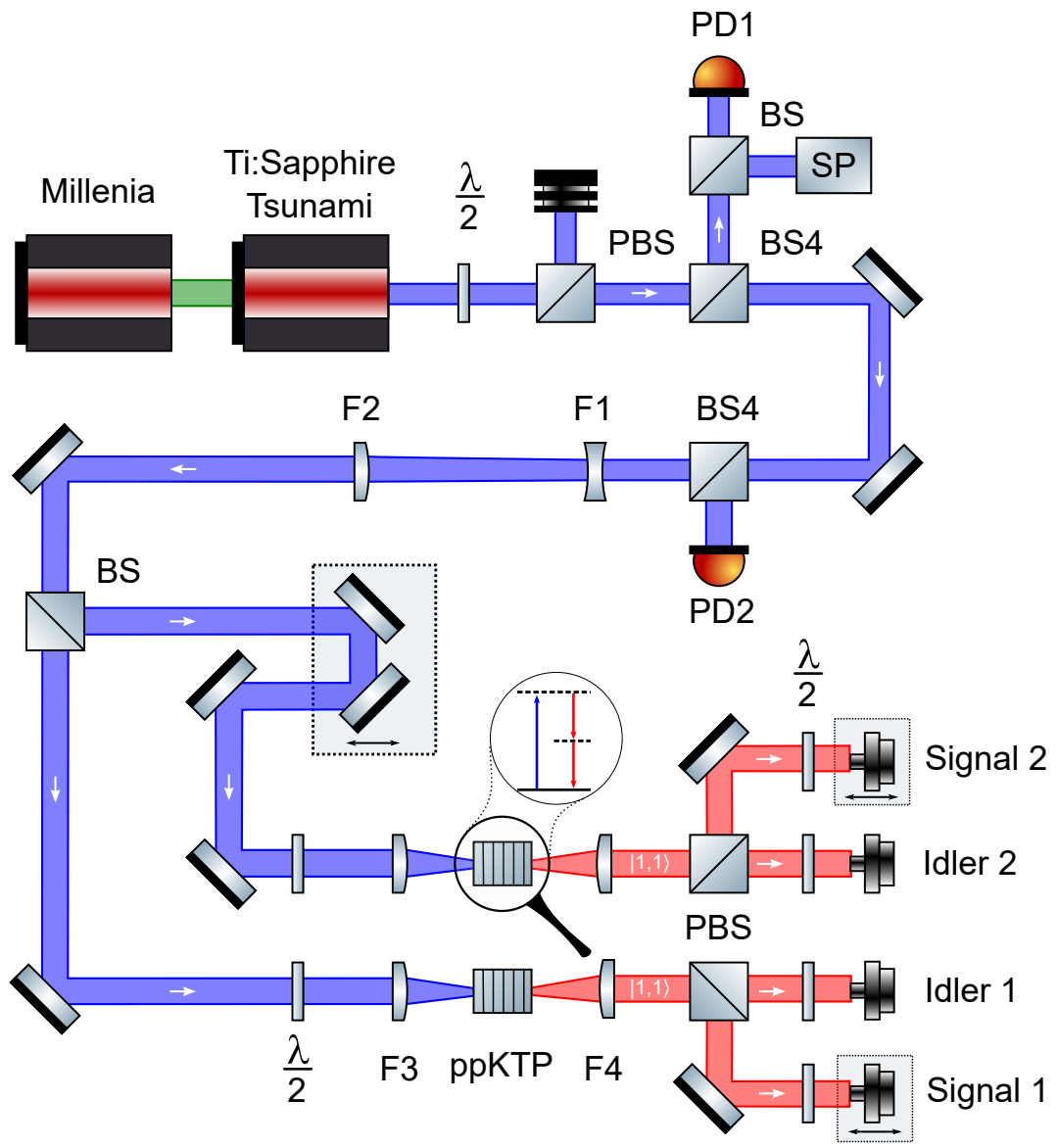

Figure 4.1 The single-photon source. The average power of the pulsed Ti:Sapphire laser is regulated by a half-waveplate $\left(\frac{\lambda}{2}\right)$ and a polarizing beam splitter (PBS). The laser beam properties are continuously checked using a photodiode (PD2) and a spectrometer (SP) by picking off some of the light with a $96: 4$ beam sampler (BS4). Another photodiode (PD1) is a fast trigger photodiode. After a beam expander (F1 and F2) the light is split by a $50: 50$ beam splitter into two paths. Each path leads to a ppKTP crystal to generate two-photon states. The light is focused into these crystals with lens F3 and collimated with F4. A PBS is used to separate the photons from the pair and then the polarization of each individual photon is adjusted before they are injected into a polarization-maintaining (PM) fiber. The fiber couplers for the signal photons and two mirrors of the $2^{\text {nd }}$ source are placed on a linear translation stage to control the temporal overlap. The long-pass optical filter to remove the pump light and an optional spectral bandpass filter after the crystals are not shown. 
to both the heralding efficiency as well as the photons purity [114]. The beam expander consists of two lenses: $\mathrm{F} 1=-75 \mathrm{~mm}$ and $\mathrm{F} 2=150 \mathrm{~mm}$.

After the beam expander, the pump beam is split into two paths, each leading to a separate nonlinear crystal. The first path goes straight through to the beam splitter (to the lower crystal in Fig. 4.1). The laser light is focused onto the nonlinear crystal with a lens (F3: $150 \mathrm{~mm})$. A second lens (F4: $200 \mathrm{~mm}$ ) is used to collimate the generated single photons. Two longpass filters (FELH1200, Thorlabs) are used to filter out the laser light.

The nonlinear crystal is a $2 \mathrm{~mm}$ periodically poled KTP crystal (ppKTP, Raicol Crystals) with a poling period of $46.2 \mu \mathrm{m}$. The crystal is placed on a five-axis stage (New focus 9081, Newport) to accurately position the crystal with respect to the pump beam. This crystal is cut of type II SPDC and therefore produces the signal and idler modes with an orthogonal polarization

$$
|\psi\rangle=\sqrt{1-\mu^{2}} \sum_{n=0}^{\infty} \mu^{n}\left|n_{\mathrm{s}}, n_{\mathrm{i}}\right\rangle,
$$

where $n$ denotes the number of photons and the prefactor $\mu$ depends on both the nonlinearity of the crystal and the pump power. The two modes are referred to as signal $\left(n_{\mathrm{S}}\right)$ and idler $\left(n_{\mathrm{i}}\right)$. As discussed in Chapter 3 , each SPDC source produces squeezed light of which one is close to a Fock state when the other mode is used as a herald. At low pump powers, the effect of multiphoton states on the purity is limited.

After the collimation lens, a polarizing beam splitter is used to couple each individual mode into a polarization-maintaining (PM) fiber using a fiber coupler (F810FC-1550, Thorlabs). The fiber coupler of the signal photon is placed on a linear motorized stage (SLC-2475, Smaract GmbH), which is used to tune the temporal overlap of the photons in the experiments.

The second path of the beam splitter goes to the second crystal. This path is an almost identical copy of the first photon source, the only difference being the motorized linear stage (SLC-2475, Smaract GmbH) before the second crystal. This stage allows to control the temporal overlap between the generated photons between both crystals.

Once the photons have propagated through the experiment, they are routed towards the detectors. There are $14 \mathrm{NbN}$ superconducting nanowire single-photon detectors (SNSPD, Photon Spot). They have a detection efficiency of around $90 \%$ and a dead time on the order of $50 \mathrm{~ns}$. The detection efficiencies depend on the polarization [148, 149], hence the photons go through a fiber polarization controller (FPC560, Thorlabs) before entering the cryostat. The readout electronics of the detectors is a combination of PhotonSpot electronics and self-made electronic circuits [150]. A time tagger (Time tagger ultra, Swabian $\mathrm{GmbH}$ ) is used to read out the detector clicks and to apply Boolean logic to extract the coincidence clicks of the detectors and from the trigger pulses from photodiode (PD1) if necessary. 


\subsection{Generation rates}

The setup is designed to generate many single photons simultaneously with a high purity. For this, one of the photons needs to be measured to herald the presence of the other. This is needed as the photons in each individual mode of an SPDC source are thermally distributed [151], but detection of a photon in one mode projects the state of the second mode into a good approximation of a single-photon Fock state. Hence, having many counts is a necessary, but insufficient condition to operate an SPDC source as a single-photon source: the heralding efficiency should be high too.

We start with examining the single rates. SPDC generates photons probabilistically, which implies that if there is a $1 \%$ probability to generate a single photon pair, then there is a $(1 \%)^{2}$ probability for this to occur twice during the same excitation pulse. Hence, SPDC also generates higher multiphoton states. The rate $R_{n}$ to generate $n$ photons simultaneously from a single crystal is proportional to

$$
R_{n} \propto\left|\left\langle n_{\mathrm{s}}, n_{\mathrm{i}}\right\rangle\right|^{2}=\operatorname{sech}^{2}(\mu) \tanh ^{2 n}(\mu) .
$$

For $\mu \ll 1$, this can be approximated to

$$
R_{n} \propto \mu^{2 n} \propto P_{\mathrm{p}}^{n}
$$

where $P_{\mathrm{p}}$ is the power of the pump field. This power law shows that the contribution of multiphoton states, e.g. $|2,2\rangle$, is small for sufficiently low pump powers.

Figure 4.2 shows the number of counts for both $|1,1\rangle$ and $|2,2\rangle$ states as a function of pump power for the first crystal of the SPDC setup. multiphoton states are measured for 5 minutes at various pump powers in the range of 5 to $100 \mathrm{~mW}$. The multiphoton states are measured using a quasi-photon-number-resolving detector consisting of a $1: 3$ PM-fiber splitter (Opneti) with a standard SNSPD detector behind each output. The counts are corrected for the relative detection and splitting ratios. The figure shows that the source has a high generation fraction of $|1,1\rangle$ states.

The $|2,2\rangle$ states are predicted by eq. 4.3 to scale with twice the exponent of $|1,1\rangle$. This is tested by the fits (grey dashed lines) in Fig. 4.2. The fits are of the form $y_{1}=A_{1} x^{1 B}$ for the $|1,1\rangle$ states and $y_{2}=A_{2} x^{2 B}$ for the $|2,2\rangle$ states, i.e. both fits have a related exponent with the same variable $B$ as a parameter. The exponent is found to be $B=1.05$. These fits agree with both data sets and hence confirm the validity of the approximation as given in eq. 4.3.

Scaling the setup up to many photons requires more than just a high generation probability. The generation probabilities in the previous paragraphs have been calculated by the average number of pairs per excitation pulse. This is not sufficient as it is possible to detect many pairs while still losing most of the photons, leading to only a single detector click instead of two simultaneous clicks. The efficiency at which both photons of the same pair can be extracted is given by the heralding efficiency. The heralding efficiency measures how many coincidences are measured, compared to the single rates. Hence the 


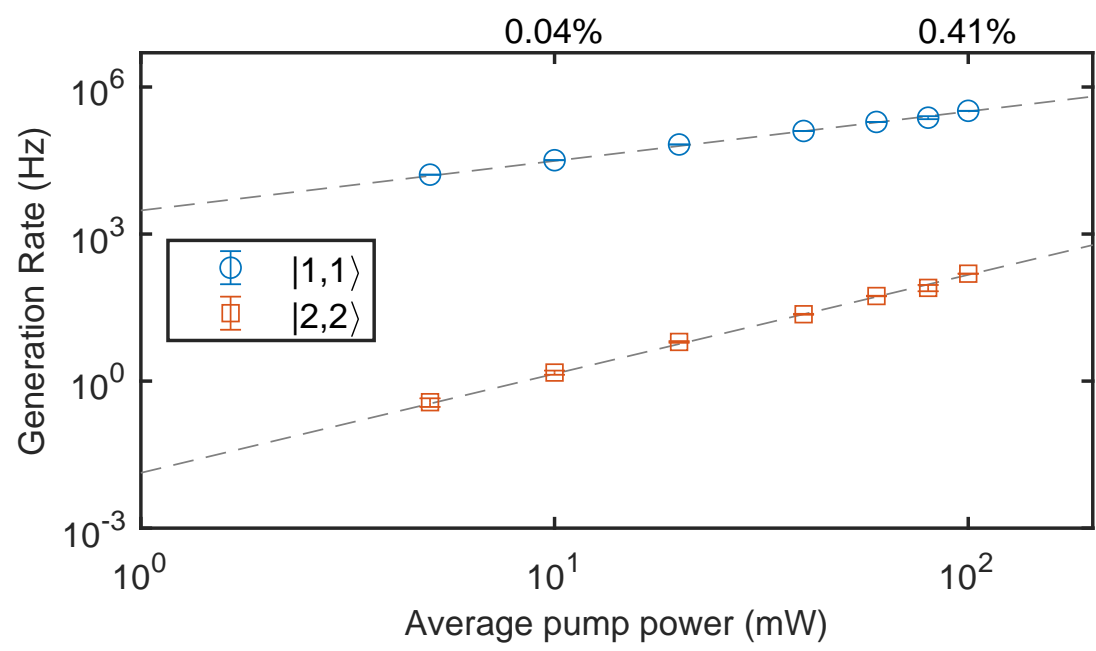

Figure 4.2 Source generation rate. The generation rate of photon pairs and a higher multiphoton state increases with pump power. The error bars indicate the standard deviation and are within the symbol size. The top axis shows the probability to generate a $|1,1\rangle$ state per excitation pulse. Each dashed grey line is an exponential fit to the data.

heralding efficiency is defined as

$$
\eta=\frac{P_{\mathrm{si}}}{\sqrt{P_{\mathrm{s}} P_{\mathrm{i}}}}
$$

with $P_{\mathrm{si}}$ the coincidence rate and $P_{\mathrm{s}}$ and $P_{\mathrm{i}}$ the single counts for the signal and idler photons, respectively. In this setup, the heralding efficiency is, on average, $46 \%$ for each crystal. The heralding efficiency is limited by the losses is each arm. The losses are dominated by the detection efficiency of around $90 \%$ and imperfect incoupling efficiency into a single-mode fiber.

\subsection{Spectral properties}

As described in section 8 , the indistinguishability of the photons generated by SPDC mainly depends on their spectral properties. For the signal and idler photons of the same pair, the indistinguishability follows from the overlap of their spectra. The indistinguishability between photons from different pairs follows from the spectral purity, i.e. the factorizability of the joint spectral intensity (JSI) [126, 127].

Figure 4.3 shows both the numerically computed as well as experimentally verified spectral properties of the multiphoton source. Figure 4.3a) shows the numerically computed JSI for our ppKTP multiphoton source. The corresponding spectral purity is $79.7 \%$. From the JSI, the ideal photon spectra are extracted as shown in Fig. 4.3b). The theoretical spectra predict $98 \%$ identical photons from the same source. 
JSI
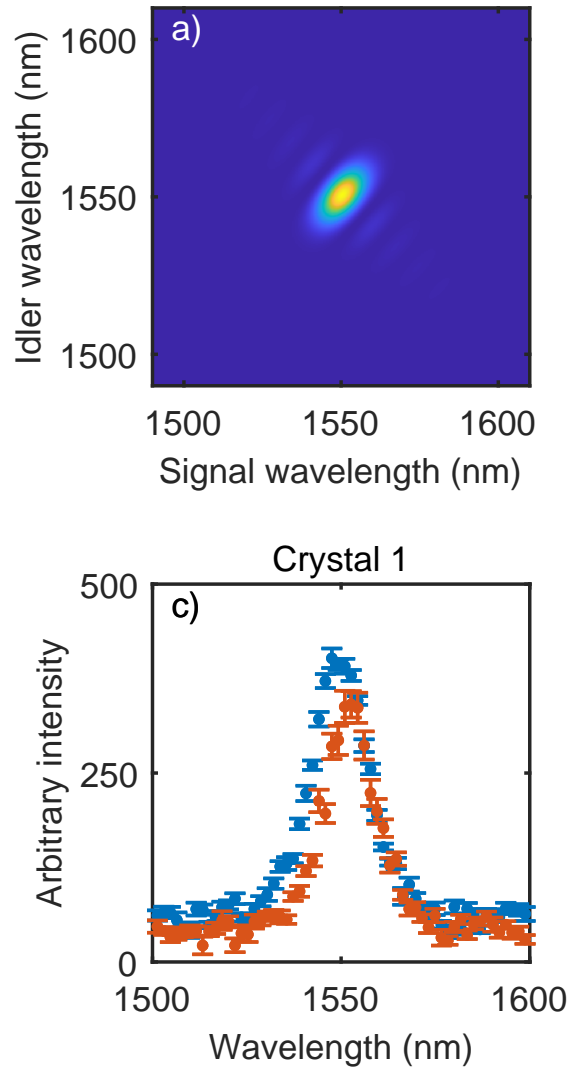
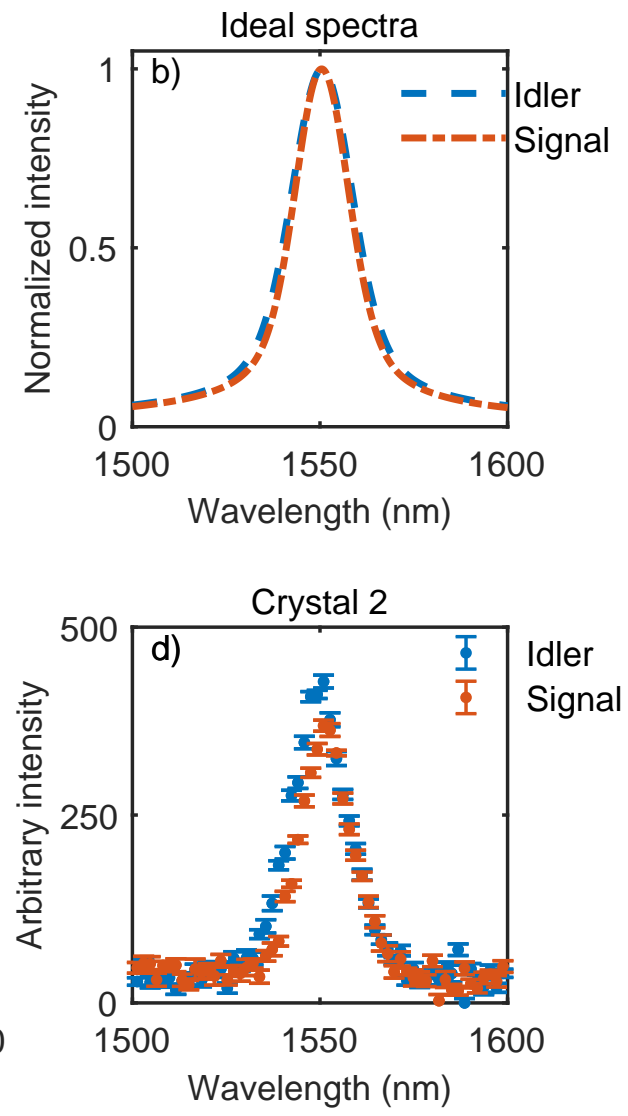

Figure 4.3 Spectral properties of the SPDC source a) Shows the theoretical JSI of the SPDC source, it corresponds to a purity of $79.7 \%$. b) Shows the expected spectra based on this JSI. Finally c) and d) show typical experimentally observed photon spectra for crystals 1 and 2, respectively. 
Figure 4.3c) and d) show the measured spectra of the photons with a standard NIR spectrometer (NIR-512, Ocean Optics). The error bars denote the standard deviation as obtained from repeated measurements. The spectra are non-degenerate as the crystals are operated at room temperature [152]. The FWHM of the photons is about $20 \mathrm{~nm}$, which is slightly less than that of the ideal spectra. Nonetheless, the high spectral overlap of the photons spectra signals a high spectral purity of the photons.

\subsubsection{Spectral purity}

Measuring the degree of (in)distinguishability between two photons is done with a HongOu-Mandel experiment [133]. In such an experiment, both photons are incident on a different input mode of a $50: 50$ beam splitter and the number of coincidences between two detectors are measured. Each detector is placed behind one of the output modes of the beam splitter. Let the matrix describing the beam splitter be given by

$$
U_{\mathrm{B} S}=\frac{1}{\sqrt{2}}\left[\begin{array}{ll}
1 & i \\
i & 1
\end{array}\right],
$$

Then the visibility of the HOM interference depends on both the 'quantum' case where both photons arrive simultaneously at the beam splitter

$$
Q=\left|\operatorname{perm}\left(U_{\mathrm{B} S}\right)\right|^{2}
$$

with perm denoting the permanent [88] and the 'classical' case where the photons arrive at a time difference longer than the photon coherence time

$$
C=\operatorname{perm}\left(\left|U_{\mathrm{B} S}\right|^{2}\right) \text {. }
$$

The total visibility is now given by [153]

$$
\begin{aligned}
V & =1-\frac{Q}{C} \\
& =x^{2},
\end{aligned}
$$

with $x^{2}=\left|\left\langle\psi_{1} \mid \psi_{2}\right\rangle\right|^{2}$.

Figure 4.4a) shows typical HOM dips for three combinations of two signal and/or idler photons as measured on the optical processor [46]. The HOM dips with the missing fourth photon are not measured since that photon is used as a herald anyway. The coincidence rates reduces when the arrival time of the first photon starts overlapping with the arrival time of the second photon. At perfect temporal overlap, the coincidence counts are minimal and the photons are maximally indistinguishable. The visibilities are around $74 \%$ for photons from different pairs and about $94 \%$ when both photons from the same pair are interfering. The full width half maximum of the HOMs dip is $\sim 300 \mathrm{fs}(90 \mu \mathrm{m})$. The observed visibilities are all about $4 \%$. less than expected from simulations. This reduction can be explained by a combination of multiphoton generation, focusing effects and other imperfections in the setup which are not included in the numerical model. 
a)

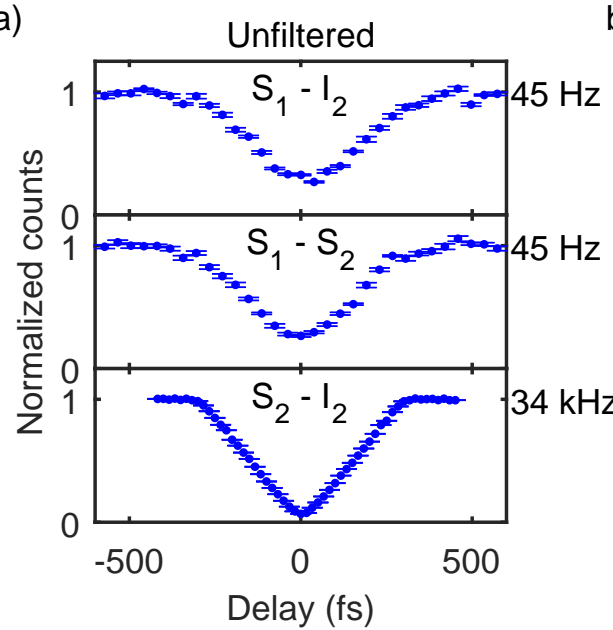

b)

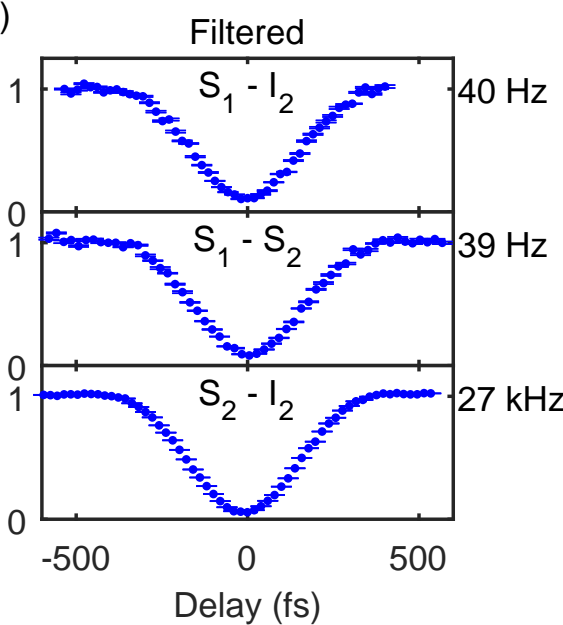

Figure 4.4 Typical HOM interference. The unfiltered HOM dips, on the left, are $73.7 \pm 0.05 \%$, $74.7 \pm 0.07 \%$ and $93.2 \pm 0.02 \%$. The right plots are the HOM dips when a $25 \mathrm{~nm}$ bandpass filter is used. In this case, the HOM visibilities are $89.4 \pm 0.08 \%, 91.8 \pm 0.05 \%$ and $94.4 \pm 0.01 \%$. All data sets are measured at $40 \mathrm{~mW}$ pump power. The error bars denote the statistical error and are within the marker size.

Figure 4.4b) shows the same HOM dips, but now with a $25 \mathrm{~nm}$ spectral bandpass filter (Edmund Optics) placed after the collimation lens. This broadband filter does not affect the rates as the minimum transmission is $\geq 90 \%$ and the filter only gently reduces the side lobes of the JSI. The coincidence rate now goes down to $90 \%$ for photons from different pairs. The HOM visibilities are still lower than the predicted HOM dip visibility of $96.8 \%$ as predicted by a model calculation. The HOM visibility of photons from the same pair did not increase significantly with spectral filtering. This indicates that other imperfections are now the main limitation for the photon purity. The outlook section explores possible explanations and solutions to improve the photon purity.

\subsection{Discussion}

The current multiphoton source is sufficient for smaller proof-of-principle experiments. It is a bright source with more than $300 \mathrm{kHz}$ two-photon coincidence rate and a high heralding efficiency of around $45 \%$. The HOM dip visibilities of $94 \%$ for photons from the same source and $75 \%$ for photons from two different sources indicate a good photon indistinguishability. Furthermore, the unfiltered HOM dips match nicely with the predicted $98 \%$ and $79.7 \%$ visibilities from simulation. A heralding efficiency of $45 \%$ with a photon purity of 0.9 results in $\alpha=\sqrt{.45} * 0.9=0.60$. This results in a maximum number of $k=11$ photons that can meaningfully interfere. This is sufficient for all experiments described in this thesis. 
The difference between the ideal and the observed HOM dips can be attributed to, among others, slight misalignment, beam astigmatism and focusing effects [114]. The first two effects induce spatial-temporal correlations in the two-photon field which reduces the purity. The focusing effect into the ppKTP crystals also reduces the purity as there are many k-vectors available in the focus and hence more downconversion frequency combinations can be phase-matched. This effect is estimated to be only a few percentage points.

There are some challenges for the current setup which might need to be addressed for future experiments. For example, the $75 \% \mathrm{HOM}$ visibility is not sufficient for some experiments (e.g., the certification measurements of Chapter 8). This could be overcome by spectral filtering. However, filtering increases the losses, meaning that the fourfold coincidence rate will decrease even more. The gentle bandpass filter of $25 \mathrm{~nm}$ could be sufficient to significantly improve the spectral purity to around $90 \%$, while not drastically reducing count rates. For future work, the source needs to be improved. Chapter 9.2 will highlight some of the current challenges and possible ways of improvement to the current setup. 


\section{ApPENDix 4}

\section{A Detector settings}

The performance of the detectors depends on the used settings. Table 4.1 gives an overview of the used settings during the experiments in this chapter. The bias currents are set to optimal detection efficiency. This corresponds to relatively high bias currents, which in turn increases the number of observed dark counts. The corresponding dark counts are typically around $150-200 \mathrm{~Hz}$, but this can be reduced by using the trigger diode as a gate for the detection events to about $3 \mathrm{~Hz}$.

The mentioned dead time is the manually set dead time of the time tagger, i.e., the time in which the time tagger does not accept a new pulse. This is necessary to prevent the time tagger to falsely detect counts on either afterpulsing or noise on the exponential tail of the pulse. The difference in detector deadtimes is important as it quantifies the sampling bias between various detectors. The procedure for determining the optimal values can be found here [154].

Table 4.1 The detector settings used in the experiments. Note that detector 13 has not yet been connected.

\begin{tabular}{l|ccc}
\hline \hline Detector & $\begin{array}{c}\text { Trigger Level } \\
\text { Volt }\end{array}$ & $\begin{array}{c}\text { Dead time } \\
\text { ns }\end{array}$ & $\begin{array}{c}\text { Bias current } \\
\mu \mathrm{I}\end{array}$ \\
\hline 1 & 0.20 & 80 & 21.5 \\
2 & 0.20 & 80 & 21.5 \\
3 & 0.20 & 55 & 21.5 \\
4 & 0.20 & 55 & 21.5 \\
5 & 0.07 & 50 & 10.5 \\
6 & 0.07 & 35 & 6.5 \\
7 & 0.07 & 50 & 9.5 \\
8 & 0.10 & 50 & 20 \\
9 & 0.06 & 25 & 6.5 \\
10 & 0.15 & 20 & 5 \\
11 & 0.10 & 25 & 10 \\
12 & 0.15 & 20 & 5 \\
13 & $/$ & $/$ & $/$ \\
14 & 0.10 & 50 & 10 \\
\hline \hline
\end{tabular}




\section{Integrated quantum processor}

Demonstrating a quantum device outperforming any classical computer on some computational task is a major milestone towards becoming a mature quantum technology. To date, two quantum platforms have made a claim that their quantum device outperforms any classical counterpart. The first is Google's superconducting system and the second is by USTC using photons [10, 25]. A second photonic experiment by USTC realized 144 modes with free-space optics and is inherently static, limiting the usability for other, future experiments [11]. A low-loss and fully reconfigurable alternative is needed if quantum photonics ever wants to become a mature and useful platform.

Integrated photonics is promised to be able to implement large and reconfigurable optical networks while maintaining low loss. There are multiple ways to implement such networks, but all networks are in essence a universal multiport interferometer that links all input modes to all output modes in a reconfigurable manner [155]. These photonic networks, or processors, have applications in many fields, such as quantum information processing [19, 20, 43, 156-165], (quantum) machine learning [26, 166$168]$ and radio-frequency signal processing [169, 170]. The quest is for a large low-loss precisely programmable integrated photonic processor.

An integrated photonic quantum processor should fulfill various requirements. First and foremost, it should preserve the quantum nature of the injected photons, i.e. the processor should not induce decoherence. For photons, decoherence corresponds to photon loss and an increased distinguishability between photons. Hence, a processor should be low-loss and not unintentionally change any internal degree of freedom of the photons. Furthermore, in order for the network to be universal: all input modes and output modes should be connected and any unitary transformation from input to output modes should be programmable. There are many different platforms to implement such networks $[45,87,155]$. Table 5.1 provides an overview of reported integrated photonic processors in literature.

This chapter introduces our scalable 12-mode integrated silicon nitride optical processor. We characterize the processor's losses and its performance for both classical and quantum light. Specifically, both the fidelity at which the transfer matrices are implemented and the extent to which photons preserve their quantum nature when propagating through this network is studied. All experiments in this thesis are performed with this $12 \times 12$ photonic processor.

\subsection{The photonic processor}

The processor is a forward-only network of stoichiometric silicon nitride $\left(\mathrm{Si}_{3} \mathrm{~N}_{4}\right.$, or $\mathrm{SiN}$ in short) asymmetric double-stripe waveguides with the TriPleX technology [176]. A major advantage of $\mathrm{SiN}$ waveguides is the low loss per functionality [163]. In our case, the waveguides are designed for $1550 \mathrm{~nm}$ and have propagation losses are as low as $0.1 \mathrm{~dB} / \mathrm{cm}$ 
Table 5.1 Overview of photonic networks. The platforms are silicon on insulator (SOI), silicon nitride $(\mathrm{SiN})$, femtosecond laser writing (fs lw). Not all reported processors state the fidelity at which the network operates or the total transmission efficiency.

\begin{tabular}{l|lllll}
\hline \hline Platform & Channels & Fidelity & Transmission & Wavelength & Reference \\
\hline SiN & $4 \times 4 \dagger$ & - & 0.16 & $1555 \mathrm{~nm}$ & {$[171]$} \\
SOI & $4 \times 4$ & - & circulating & $1550 \mathrm{~nm}$ & {$[170]$} \\
SOI & $4 \times 4$ & - & 0.20 & $1550 \mathrm{~nm}$ & {$[157]$} \\
SiN & $4 \times 4$ & - & $0.45^{* *}$ & $1550 \mathrm{~nm}$ & {$[172]$} \\
fs LW & $5 \times 5$ & fixed & 0.21 & $789 \mathrm{~nm}$ & {$[20]$} \\
fs LW & $5 \times 5$ & fixed & 0.30 & $785 \mathrm{~nm}$ & {$[21]$} \\
UV laser & $6 \times 6$ & fixed & - & $830 \mathrm{~nm}$ & {$[19]$} \\
Silica & $6 \times 6$ & $0.94^{+\dagger}$ & 0.58 & $808 \mathrm{~nm}$ & {$[156]$} \\
fs LW & $6 \times 32$ & - & 0.45 & $785 \mathrm{~nm}$ & {$[165]$} \\
SiN & $8 \times 8$ & $0.91^{\ddagger}$ & 0.40 & $1550 \mathrm{~nm}$ & {$[163]$} \\
SiN & $9 \times 9$ & fixed & - & $780 \mathrm{~nm}$ & {$[173]$} \\
Si & $4 \times 12$ & fixed & $0.09^{* * *}$ & $1550 \mathrm{~nm}$ & {$[162]$} \\
Si & $26 \times 26$ & $0.98^{\dagger+}$ & 0.16 & $1570 \mathrm{~nm}$ & {$[159]$} \\
fs LW & $13 \times 13$ & fixed & 0.13 & $785 \mathrm{~nm}$ & {$[54]$} \\
Si & $16 \times 16$ & $-\ddagger \ddagger$ & - & $1545 \mathrm{~nm}$ & {$[174]$} \\
Si & $240 \times 240$ & $-*$ & 0.11 & $1500 \mathrm{~nm}$ & {$[175]$} \\
SiN & $12 \times 12$ & $0.98^{\ddagger}$ & 0.57 & $1550 \mathrm{~nm}$ & This work \\
\hline \hline
\end{tabular}

${ }^{\dagger} 8$ modes when spectrally multiplexed

${ }^{++}$After iterative optimization

$\ddagger$ Amplitude fidelity

㧊 Only provides state fidelities

* Only on/off switching

${ }^{* *}$ Excluding $-15 \mathrm{~dB}$ loss per facet

*** Losses include on-chip photon generation 


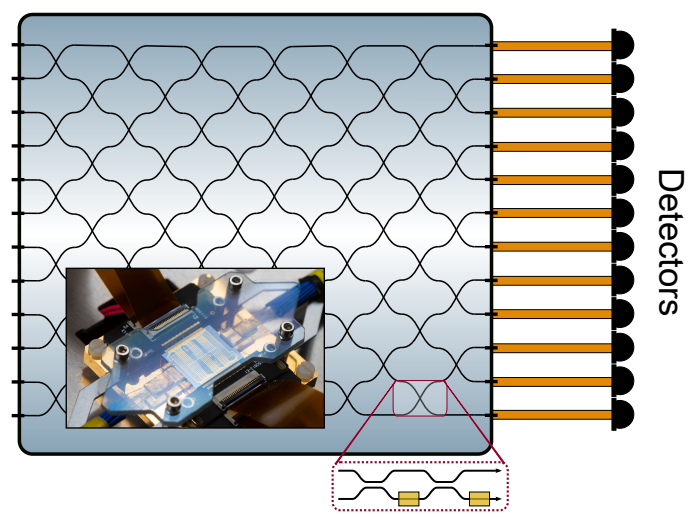

Figure 5.1 A schematical representation of the processor. The processor has 12 input modes (left) and output modes (right). It consists of a forward-only mesh of $2 \times 2$ unit cells as shown at the bottom. Each unit cell has two heaters (yellow block) in a Mach-Zehnder interferometer. The positions A, B and C correspond with those mentioned in Table 5.2.

and $0.5 \mathrm{~dB}$ per facet. The total losses are $2.5 \pm 0.2 \mathrm{~dB}$ per photon, depending on the used modes.

Figure 5.1 shows a schematical representation of the processor. Light incident at any of the 12 input modes can be arbitrarily routed to any arbitrary output configuration by appropriately tuning the networks of the unit cells. Each unit cell implements a pairwise interaction between two adjacent modes with a tunable beam splitter [177]. These beam splitters are realized using a balanced Mach-Zehnder interferometer and two heaters. Each heater is a $1 \mathrm{~mm}$ platinum bar that can locally change the optical path length by thermally changing the refractive index in the waveguide. The processor has a total of 66 unit cells and 132 heaters to allow full reconfigurability.

\subsection{Tunability}

The precise control of the processor is important to ensure it programmability. Therefore, we investigate the fidelity of a set of matrices. Now we put the focus on the cross-talk. The heaters can be a double-edged sword. On the one hand, the heaters allow the processor to be fully reconfigurable, but on the other hand, they limit the tunability by electric and thermal crosstalk. This tunability can be improved by adjusting each heater for its crosstalk with its neighbors.

The first characterization of the tunability is by investigating the fidelity at which transfer matrices can be implemented that describe the relation from input to output modes. For this, classical CW light from a $1550 \mathrm{~nm}$ superluminescent diode (Thorlabs $\mathrm{S} 5 \mathrm{FC} 1005 \mathrm{P}$ ) is injected into the input modes and an array of calibrated photodiodes (Thorlabs FGa01FC) are used to detect the output signal. The amplitude fidelities are 
a)
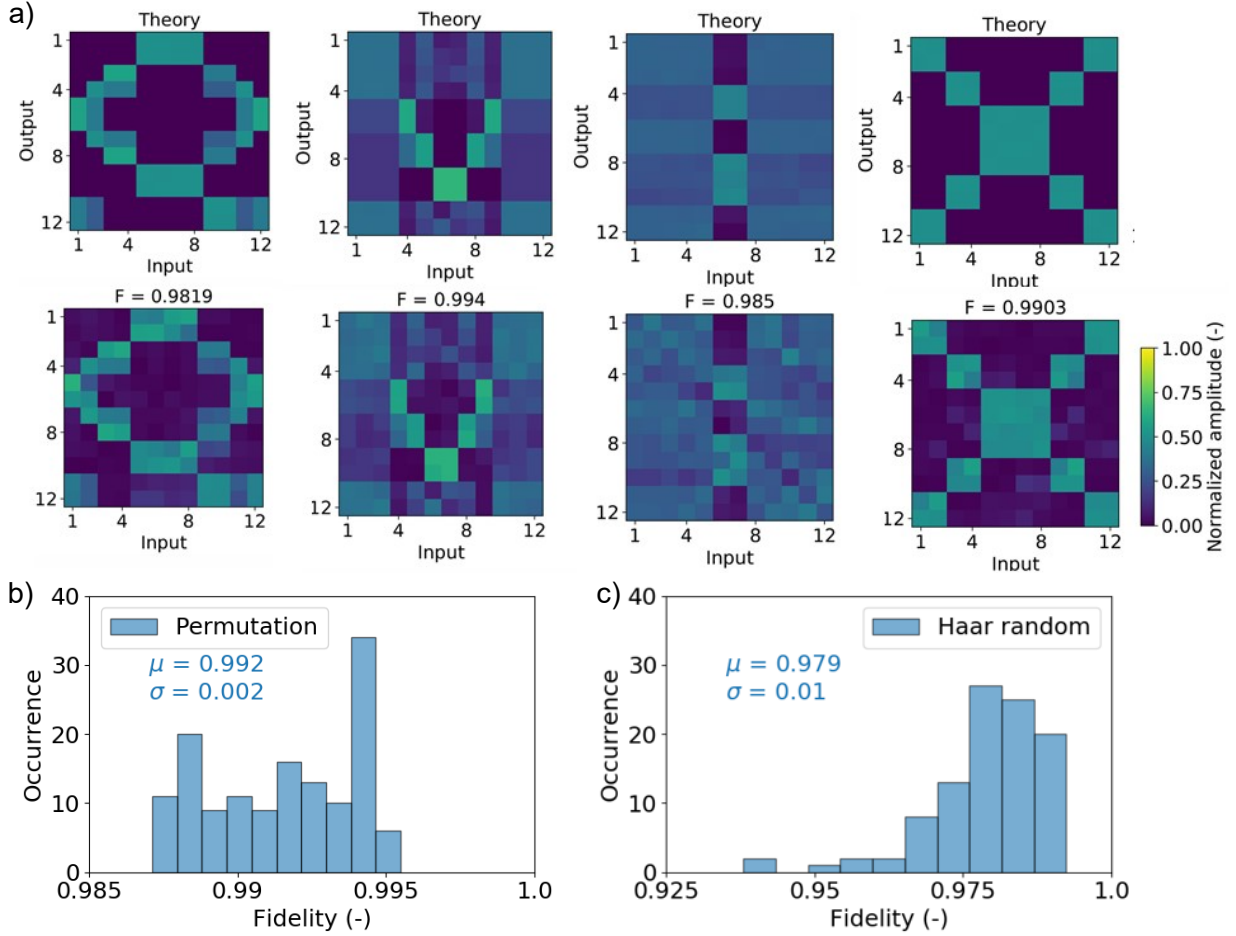

Figure 5.2 Amplitude fidelities. a) the top row shows four transfer matrices whose amplitudes spell out 'Quix'. The bottom row shows the experimental realization of these target matrices with the associated amplitude fidelities on top. b) A histogram of amplitude fidelities for 150 permutation matrices with an average fidelity of $F_{\text {Perm }}=0.992 \pm 0.002$, where the error margin denotes the standard deviation. c) The histogram of amplitude fidelities of 100 Haar random matrices. The average fidelity is $F_{\text {Haar }}=0.98 \pm 0.01$, where the error margin again denotes the standard deviation. 
defined as $F=\frac{1}{N} \operatorname{Tr}\left(\left|U_{\text {set }}^{\dagger}\right| \cdot\left|U_{\text {get }}\right|\right)$, where $U_{\text {get }}$ denotes observed transfer matrix, $U_{\text {set }}$ is the target transfer matrix and the absolute signs indicate the element-wise absolute value of the matrix elements.

Figure 5.2 visualizes that arbitrary transformations can be realized with a high amplitude fidelity. Figure 5.2a) shows four examples of transfer matrices that spell 'Quix'. The top row shows the ideal matrix and the lower row the experimentally realized matrices together with the fidelity. Figure 5.2b) and 5.2c) show the distribution of fidelities for two different classes of matrices, namely permutation and Haar random matrices, respectively. Both classes of matrices have a high average fidelity of around 0.985 and the lowest observed fidelity is still above 0.93 .

\subsubsection{Preservation of quantumness}

The processor is fully tunable and has low losses. The remaining requirement of the network is that it preserves the quantum properties of the photons as they propagate through the network. Table 5.2 shows that the HOM interference visibility remains constant as the photons propagate through the network. The locations $\mathrm{A}, \mathrm{B}$, and $\mathrm{C}$ correspond with those indicated in Fig. 5.1 and the position of the linear stage of the signal photon

The processor should not just connect all input to all output modes with full tunability, it should also preserve the quantum nature of the photons, i.e. the photons should maintain their distinguishability as they propagate through the processor. For the quantum characterization, a $2 \mathrm{~mm}$ ppKTP crystal was pumped with $150 \mathrm{fs}$ pulses to generate collinear frequency-degenerate single-photon pairs, where each photon has an orthogonal polarization. The photons are gently filtered with a $25 \mathrm{~nm}$ bandpass filter, which results in photons that are approximately $94 \%$ indistinguishable. The generated photons are routed to the chip via polarization-maintaining (PM) fibers. The output modes are connected to superconducting nanowire single-photon detectors using PM fibers. More detail on the setup can be found in chapter 4 .

Figure 5.3 shows the quantum matrix characterization for a randomly chosen $12 \times 12$ unitary matrix $[178,179]$. There is a clear correspondence between the matrix amplitudes. The reconstructed matrix phases are different from the target matrix, however, most of these phases correspond with matrix elements that have a low amplitude. The observed amplitude fidelity is 0.878 and full matrix fidelity $F=\frac{1}{N}\left|\operatorname{Tr}\left(U_{\text {set }}^{\dagger} \cdot U_{\text {get }}\right)\right|=0.61$. One limiting factor is the broad photon spectra, which is approximately $25 \mathrm{~nm}$ FWHM. The broad spectral width of the photons makes them prone to the wavelength dependence of the processor. For this reason, the amplitude fidelity measured above with classical narrow-band light is higher and therefore high quantum fidelities should be reachable with picosecond single photons.

\subsubsection{Thermal photon source}

Figure 5.4a) shows the observed dark count rates on 12 SNSPDs when all heaters are tuned to the same voltage simultaneously. Each detector is placed behind one of the 

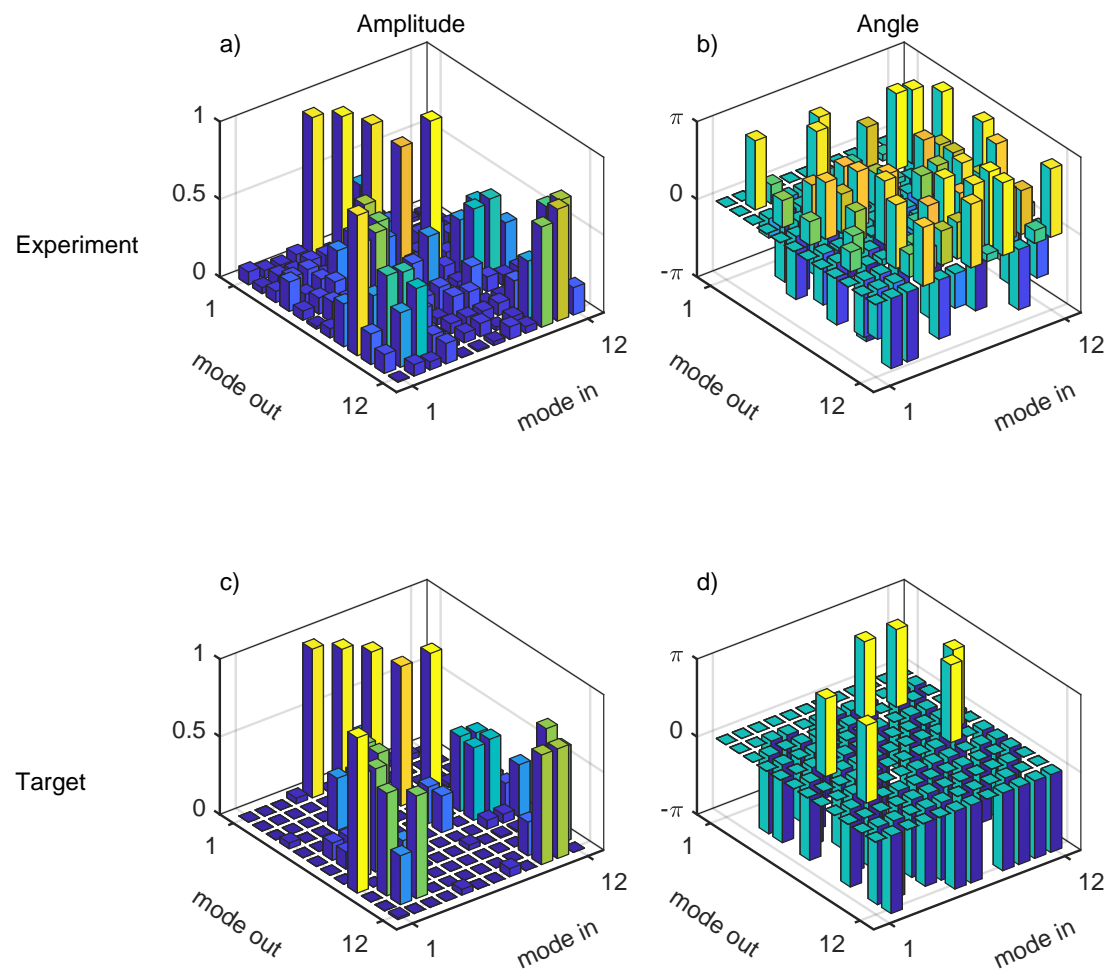

Figure 5.3 Quantum matrix reconstruction. On the top row, a) and b) indicate the experimental observed matrix elements amplitudes and phases, respectively. The bottom row shows the target matrix elements. The corresponding fidelity is 0.878 .

Table 5.2 Preservation of quantum nature. The HOM visibilities and stage positions do not change as the photons interfere at beam splitters further in the chip. The positions A, B and C correspond with those indicated in Fig. 5.1. The error bars denote the statistical error.

\begin{tabular}{l|cc}
\hline \hline Position & $\begin{array}{r}\text { Stage } \\
(\mathrm{mm})\end{array}$ & Visibility \\
\hline $\mathrm{A}$ & 4.678 & $93.0 \pm 0.04 \%$ \\
$\mathrm{~B}$ & 4.678 & $93.0 \pm 0.03 \%$ \\
$\mathrm{C}$ & 4.675 & $93.2 \pm 0.06 \%$ \\
\hline \hline
\end{tabular}




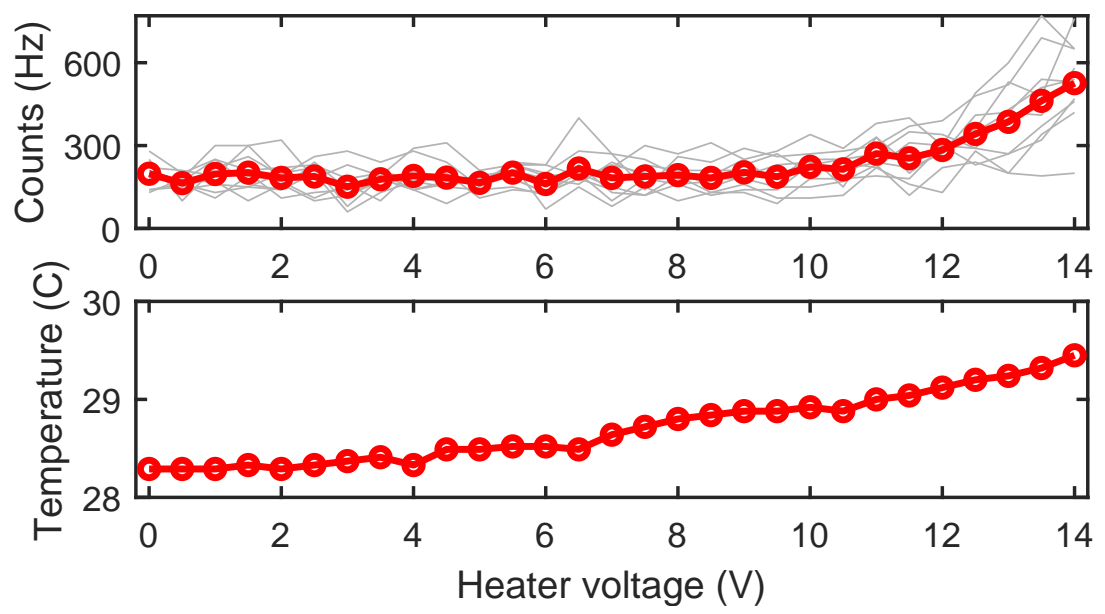

Figure 5.4 Dark counts with heater voltage Top: the measured dark counts for each detector (grey line) and the average (red) when all heaters on the chip are simultaneously set to a certain voltage. No photons were injected into the chip. Bottom: the change of temperature on the chip when all heaters are set to increasingly higher voltages.

output modes of the chip. All heaters are simultaneously swept from $0 \mathrm{~V}$ to $14 \mathrm{~V}$, which is the maximum voltage for each heater. The dark counts remain around $200 \mathrm{~Hz}$ up to $10 \mathrm{~V}$. Above this value, the dark counts increase to an average of $526 \mathrm{~Hz}$. Typical operation of the chip requires an average heater voltage of $8.5 \mathrm{~V}$ in the DMPK experiment, see Chapter 6.

Figure 5.4b) shows how the chip temperature slowly increases from the default $28.3^{\circ} \mathrm{C}$ when the thermal load is increased. The nonlinear behavior between the increase of temperature and the increase of dark counts can probably be understood by the nonlinear relation between the temperature and (spectral) radiance of Planck's law of black body radiation.

\subsection{Conclusion}

In conclusion, we have reported a low-loss fully reconfigurable 12-mode universal photonic processor based on silicon nitride technology. The processor operates at $1550 \mathrm{~nm}$ with an average loss of $2.5 \mathrm{~dB}$. It has 132 heaters with which it can implement any transfer matrix with an amplitude fidelity of $F=0.98 \pm 0.01$ and $F=0.87$ for single photons. Furthermore, the processor preserves the quantum nature of the photons, making it a suitable plug-and-play platform for quantum technology. Even higher-fidelity quantum implementations are feasible with some additional straightforward improvements. 



\section{Observation of open scattering channels}

Wave transport through scattering media is ubiquitous in nature and technology. Its physics is essential in electron and phonon transport in quantum dots and nanowires [180, 181], conductance fluctuations in electron transport [182], optical transmission in multimode fibers [183, 184], the theory of acoustic waves [185-187], and fluctuations in light transport [188-190]. An understanding of scattering physics, together with adaptive optical technologies, allows us to exploit, scattering for various applications such as wavefront shaping [80], physical unclonable functions [191-193], communication [194], imaging [195-198] and to build spectrometers [199-201].

Open channels have a pivotal role in transport through disordered systems [47]. These open channels are eigenmodes of the transmission matrix with full transmission through an otherwise opaque medium $[49,50]$. It is not just the case that open channels can exist - one can easily imagine encountering a fully transmissive mode with an exponentially small probability -, but rather that transport is dominated by fully closed and fully open channels. This is because the distribution of transmission eigenvalues (more precisely, that of the singular-values) is bimodal, with one peak at low transmission values and one peak at high values, as indicated in Fig. 6.1a). This bimodal distribution is considered to be one of the most spectacular predictions of random matrix theory [47], and remains as yet unconfirmed in direct experiments.

Technologically, open channels underlie many of the applications of scattering systems. For example, it is possible to increase the transmission to (near) unity in a disordered medium by coupling the input light into one of the open channels. This allows for lossless transmission through a very lossy medium. One can take this one step further by using a spatial light modulator to 'undo' the scattering and create a focus behind the scattering sample [80]. As the transmission matrix is not unitary, simply applying some unitary matrix with the spatial light modulator does not guarantee a high transmission; a high transmission is only possible when open channels exist [52,53]. Furthermore, conductance fluctuations in optical or electronic transport intricately depend on the existence of the bimodal distribution and its higher-order moments [182, 188].

Despite the central role of open channels in transport through disordered media, only indirect signatures for the existence of the bimodal distribution with the open channels have been provided [187, 202-204]. The limiting factor in measuring the bimodal singular values distribution in scattering media is the difficulty to individually probe and measure all of the modes of the system [51-53]. This can be understood by realizing that longrange mesoscopic correlations at the output build-up as the light gets randomly scattered and interferes with itself. Missing modes imply losing this long-range order, which ultimately leads to uncorrelated Marcenko-Pastur (MP) statistics. The open channels are only observable when the number of controlled modes must be $>\approx 95 \%$ of the total

The content of this chapter is adapted from the following submitted paper: R. van der Meer et al., arXiv:2110.04380. 
a)

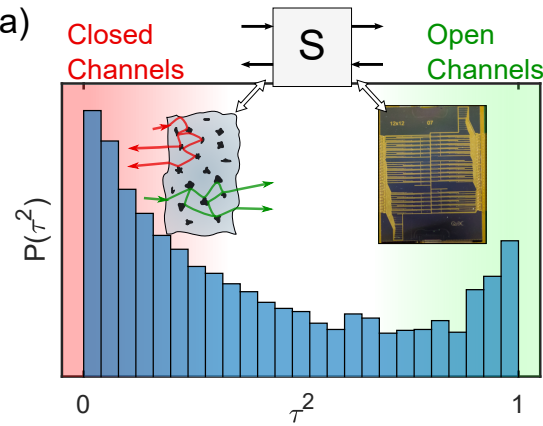

b)

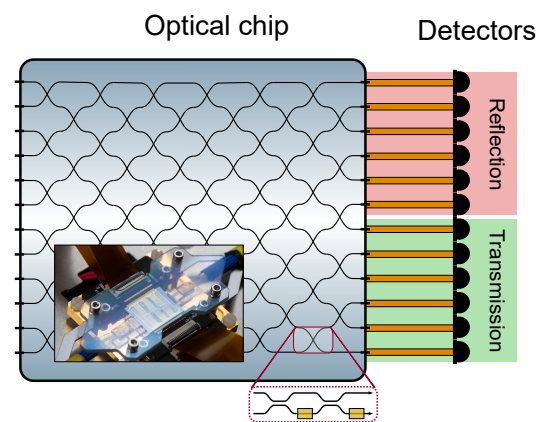

Figure 6.1 Mapping a scattering system with open channels.a) The bimodal distribution of the singular values $\tau$ of the transmission matrix of a loss-free scattering system is described by the DMPK distribution. The peak near $\tau^{2}=0$ (left, on red background) is caused by the closed channels and the peak near $\tau^{2}=1$ (right, green background) is caused by the open channels. The inset shows how light incident on a scattering medium tends to be fully reflected (red), but some eigenmodes have a near-unity transmission (green). This system can be completely modeled by a scattering matrix $\mathbf{S}$ that we simulate on a programmable optical network. b) A schematic of the programmable optical network with 12 input and output modes that implements the scattering matrix $S$. The top 6 output modes (red) correspond to the reflection modes of $S$ and the bottom (green) ones correspond to the transmission modes. On the bottom, a unit-cell of the network is shown. Each cell consists of two 50:50 splitters and two thermo-optical elements for tunability.

number of channels [53, 205]. Despite considerable effort, experimental access to a sufficient fraction of modes has so far not been achieved. Reimposing unitarity on only the observed modes amounts to the assertion that the observed set of modes is decoupled from all others, which is unjustified in the experimental situation of a scattering system. Consequently, the second peak in the singular-value distribution has not unambiguously been observed yet.

In this chapter, we report experimental proof of the existence of the bimodal distribution with its open channels from a telltale high-transmission peak in the singular-value distribution (SVD). We do so by mapping a scattering medium with exactly 6 input and 6 output channels to a $12 \times 12$ scattering matrix implemented on a linear integrated optical processor. We experimentally characterize the full transmission matrix using two-photon interference as a robust readout technique. From this, we observe the bimodal transmission singular value distribution. Profiting from the superb access and control over all modes given by an integrated photonic processor, we observe that open channels are only visible in the experimental eigenvalue distribution when all modes are considered. 


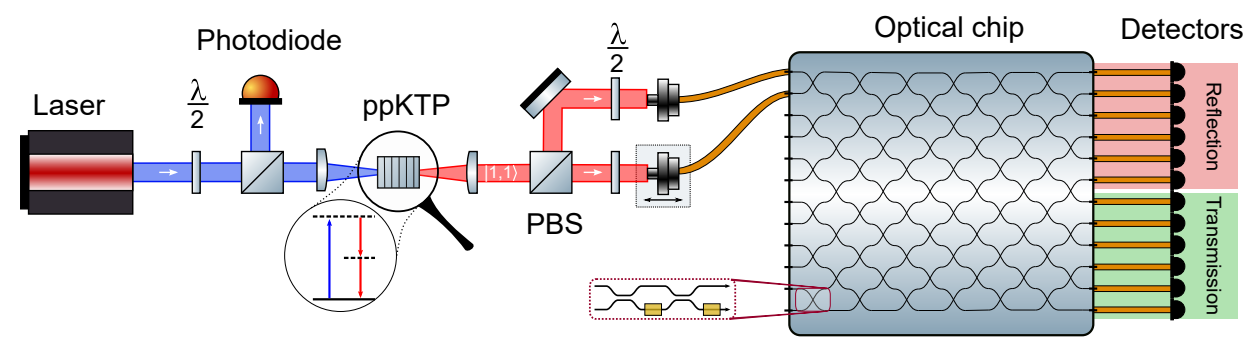

Figure 6.2 A sketch of the setup. A pulsed laser is used to generate pairs of photons in a ppKTP crystal. The photons have orthogonal polarization and are separated by a polarizing beam splitter and subsequently coupled into a PM fiber which is connected to the optical network. After the optical network, the photons go through an SMF to the single-photon detectors via a fiber polarization controller (not shown). To guarantee temporal overlap of the photons, one of the fiber couplers is placed on a linear stage. A beam sampler is used to monitor the power using a calibrated photodiode and the pump beam is filtered out after the ppKTP crystal (not shown).

\subsection{Setup}

We simulate our diffusive system on such a state-of-the-art 12-mode one-way integrated photonic processor, shown in the inset of Fig. 6.1a) and schematically in Fig. 6.1b) [46]. On this network, an entire scattering matrix $\mathbf{S}$ is implemented, where the first six output modes are treated as 'reflection' modes and output modes $7-12$ as transmission modes.

The setup, shown in Fig. 6.2, generates pairs of photons in a Type-II degenerate spontaneous parametric downconversion (SPDC) source with a $2 \mathrm{~mm}$ periodically poled potassium titanyl phosphate (ppKTP) crystal. This crystal is pumped by a femtosecond mode-locked Ti:Sapphire laser (Tsunami, Spectra Physics) which emits light at $775 \mathrm{~nm}$ and has a linewidth of $5.5 \mathrm{~nm}$. The generated photons are separated by a polarizing beam splitter and then injected into a polarization-maintaining fiber which routes these photons to the $\mathrm{Si}_{3} \mathrm{~N}_{4}$ integrated photonic network (Quix Quantum BV). One of the fiber couplers is placed on a linear stage (SLC-2475, Smaract $\mathrm{GmbH}$ ) to achieve temporal overlap of the photons inside the chip. The optical chip consists of 12 input and 12 output modes and is fully tunable [46]. Once the photons have propagated through the chip, they are routed towards the superconducting nanowire single-photon detectors (SNSPD) (Photon Spot). The relative detector efficiencies are obtained by sequentially sending the photons from input mode 1 to each output mode by implementing permutation matrices on chip. The relative counts on the detectors are directly proportional to the relative losses and detection efficiencies at all output modes. A time tagger (Time tagger ultra, Swabian Instruments) is used to read out the single clicks of the detectors and trigger photodiode (TDA 200, PicoQuant) and their coincidences. The laser's output power is constantly monitored with a calibrated photodiode.

The single-photon source is pumped with $50 \mathrm{~mW}$, which results in an observed photon 
pair rate of about $210 \mathrm{kHz}$. The heralding efficiency, i.e. the probability of detecting the second photon when the first is detected, is typically around $45 \%$. The photons are $93 \%$ indistinguishable. The detector dark counts are suppressed with the trigger photodiode to an average of $3.1 \pm 0.8 \mathrm{~Hz}$.

The integrated photonic chip is a $\mathrm{Si}_{3} \mathrm{~N}_{4}$ network by Quix Quantum. The optical chip consists of 12 input and 12 output modes with a Clements-type network, linking all input and output modes with each other [177]. The matrices are implemented with an average fidelity of $F=\frac{1}{n} \operatorname{Tr}\left(U_{\mathrm{t}} U_{\mathrm{e}}^{*}\right)=0.69 \pm 0.07$. The losses in the chip are low $(<0.1 \mathrm{~dB} / \mathrm{cm})$ [176] and dominated by the fiber-to-chip connections which are around $20 \%$ per facet. Furthermore, any losses on the chip are uniformly distributed over the modes because of the square geometry of the chip. This is important as it allows to divide out the optical losses and describe the propagation of the photons through the chip by a unitary matrix [206].

\subsubsection{Methods}

The natural mathematical framework describing this scattering physics is random matrix theory (RMT). RMT replaces system-specific details with a scattering matrix $\mathbf{S}$

$$
\mathbf{S}=\left[\begin{array}{cc}
R & T \\
T^{\prime} & R^{\prime}
\end{array}\right],
$$

where the submatrices $T$ and $R$ are the transmission and reflection matrices, respectively. This scattering matrix contains the appropriate statistical properties of the system, while remaining agnostic to the microscopic details of the scatterer. This allows studying their physics on any system that captures these statistics.

Characterizing such a matrix only results in six singular values, which is not sufficient to accurately build up the entire bimodal distribution. However, a major advantage of this network is that it is fully reconfigurable. For this experiment, we implemented a total of 200 different scattering matrices by changing the voltages over all 132 heaters. The scattering matrices are generated by a numerical simulation of a 12-mode scattering system with appropriate settings. The simulation is based on the method of [207], as described in more detail in Appendix 6.A.

Characterisation of the scattering matrices on the network is performed by sending pairs of single photons into the network and sampling their output distribution with a battery of SNSPDs. Although it would in principle be possible to characterize the matrix with classical coherent light in an interferometrically stable setup, doing the readout with single photons has the advantage that we do not need interferometric stability of the fibers connecting the PIC network with the outside world [178, 179], a fact which arises from the phase-insensitivity of the single-photon quantum state. Hence our readout method is motivated by the quantum readout being more practical than the equivalent classical method.

The matrix amplitudes are sampled by sequentially injecting single photons into each input mode and measuring the output distribution. The photon flux is corrected for 


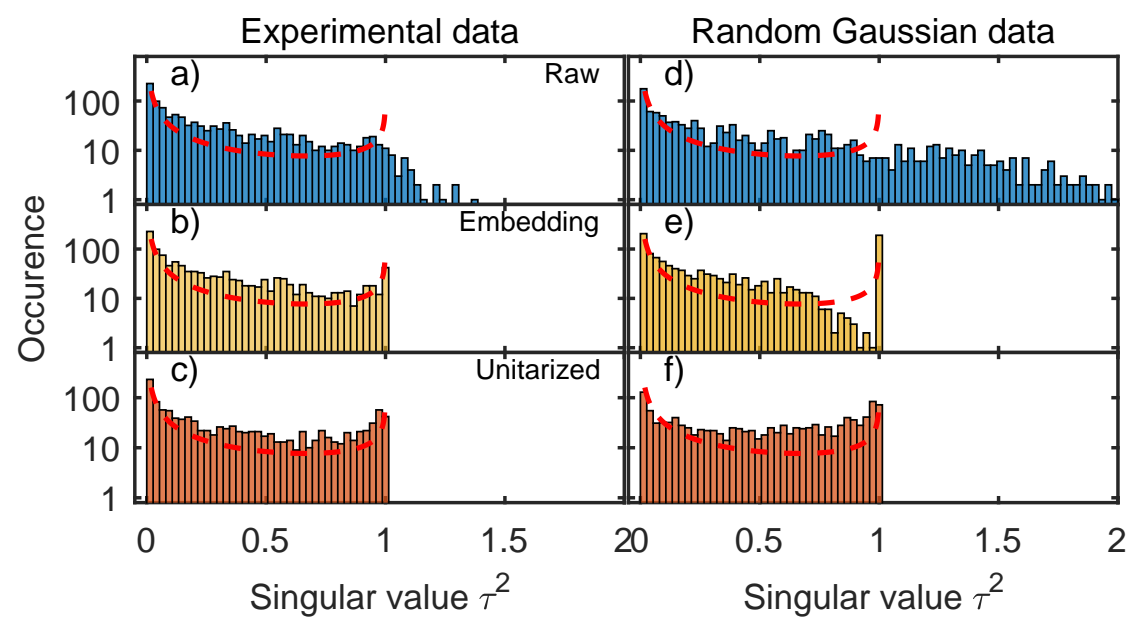

Figure 6.3 Open channels. The singular values distributions of the transmission matrix for two data sets and three different methods of processing. The left three panels correspond with the actual experimental data and the right column with data from random Gaussian matrices. The top row (a and d) shows the raw data. The middle panels ( $b$ and e) show that the bimodal distribution can be recovered by embedding the matrices, but only for the experimental data. The bottom panels (c and f) show that the unitarization of the data always results in a bimodal distribution. The unitarized Gaussian matrices lead to a symmetric distribution, whereas the unitarized experimental data show the asymmetric DMPK distribution.

known experimental fluctuations such as the variations of pump power over time, relative detector efficiencies, and output losses of the chip. The phases of the matrix elements are characterized by sequentially measuring two-photon interference in the network for a given set of combinations of two input and two output modes [133, 178, 179].

To reduce experimental measurement time, we only characterized the phases of the transmission matrix, not of the reflection matrix. The matrix amplitudes are measured for the entire $\mathbf{S}$ matrix so that the 1-photon output distribution can be normalized.

\subsection{Open Channels.}

Figure 6.3a) shows the experimentally obtained singular-value distribution of the transmission matrix. The shoulder near the singular value $\tau^{2}=1$ in Fig. 6.3a) is indicative of the expected peak, this by itself is not enough to claim observation of open channels. Ideally, energy conservation results in singular values between 0 and 1 . However, experimental noise resulted in a non-normalized $S$ matrix. The intensities of the rows sums up to $1.00 \pm 0.053$, whereas each individual column sum up to exactly 1 due to the normalisation of the measured output distribution.

The open channels are recovered in Fig. 6.3b) using a no-gain assumption by em- 


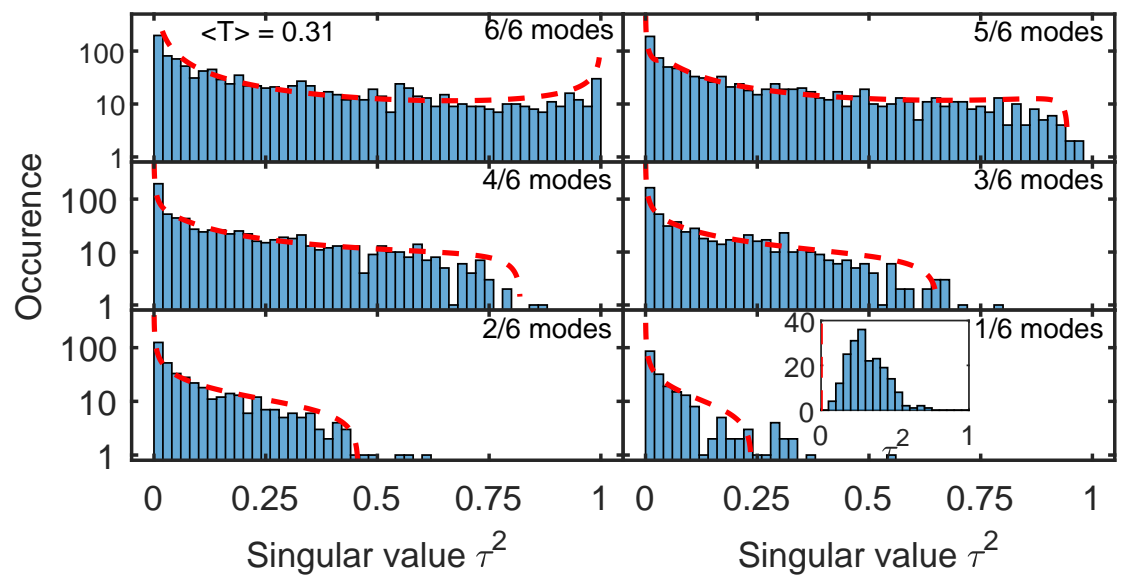

Figure 6.4 Mode Filtering. The singular value distribution of the transmission matrix for the embedded matrices. Each panel corresponds with a different number of 'observed' modes. The observed distributions match the (zero-free-parameter) predictions from Goetschy and Stone [52] well, as indicated by the red dashed lines. The insert in the last panel shows the observation and theory predictions for the situation where the control over the input channels is not reduced, a situation in which the (non-square) transmission matrix starts asymptotically resembling a random matrix. 
bedding the transmission matrix in a larger, unitary matrix. This embedding matrix can physically be understood as a matrix that also incorporates the losses and coupling to the environment [68]. We can then apply the no-gain assumption to this larger embedding matrix and again extract the new transmission matrix $T$. More details on the embedding method can be found in Appendix 6.B.

The singular value distribution now has a large and relatively broad peak at $\tau^{2}=0$ and a smaller peak at $\tau^{2}=1$ indicating the open channels. Furthermore, the distribution follows the ideal DMPK curve indicated by the red, dashed line is given by [208]

$$
\rho(T)=A \frac{g}{2 T \sqrt{1-T}}
$$

where $A$ is a zero-free-parameter scaling factor that converts the probability density function to counts. The extracted average transmission $\langle T\rangle=31 \%=\frac{l^{*}}{L}$ is close to the expected transmission (37\%). The value of the average transmission indicates that the system is approximately in the diffusive regime as $l^{*}<L$, where $l^{*}$ is the transport mean free path [209] and $L$ the characteristic system size. The average dimensionless conductance $g=\sum_{i} \tau_{i}^{2}=1.9 \pm 0.5$, where the uncertainty indicates the standard deviation over all 200 independent conductance values.

Another data processing option is to impose unitarity, or energy conservation on the experimentally reconstructed scattering matrices. This is shown in Fig. 6.3c). This panel shows that the bimodal behavior with the open channels is again recovered.

It is tempting to simply unitarize the experimental scattering matrix to mitigate the noise. However, because the essence of observing open channels is to not miss any modes, applying unitarization to a noisy scattering matrix amounts to imposing the desired solution of the data as it artificially imposes (long-range) correlations in the transmission matrix. However, these new correlations do no longer correspond with a DMPK system, but with that of a chaotic cavity [48]. To emphasize this effect, we will compare our data analysis procedure of our data with that of artificial data, generated by computer from random complex Gaussian matrices with the same mean and variance as observed in our experimental data. This artificial data is shown in Fig. 6.3d).

Figure 6.4e) shows the singular value distribution of the random Gaussian data after applying the same embedding procedure of Fig. $6.3 \mathrm{~b}$ ). The high peak at $\tau^{2}=1$ almost reaches 200 and is the result of the renormalization of almost all 200 Gaussian matrices. Despite the presence of the high peak at $\tau^{2}=1$, the distribution still clearly does not follow the one expected from DMPK statistics, which proves that it is possible to differentiate between actual and random data when the embedding procedure is used. This highlights the robustness of our data processing.

Finally, Fig. 6.3f) confirms the insight that the unitarization of random Gaussian matrices indeed results in a bimodal distribution of the singular values. The resulting distribution has lost its asymmetry, but this subtlety is eluded in experiments with limited data to sample the distribution. 


\subsection{Mode filtering.}

The observation of open channels in Fig. 6.3 is only possible because of the complete control over the number of modes. Missing out even one mode is already sufficient to hide the open channels [52]. Figure 6.4b-f) shows that the correlations inside the transmission matrix disappear when the fraction of observed modes at both the input and the output is decreased. The resulting filtered distributions match the predictions of Goetschy and Stone [52], which are indicated by the red dashed lines. This emphasizes the demanding restriction that almost all modes must be included in measurement in order to observe the open channels.

When the fraction of either controlled input or output modes is decreased, then the singular values will become uncorrelated and the open channels again disappear. The singular-value distribution will asymptotically follow the Marcenko-Pastur (MP) law since this describes the singular values of random rectangular Gaussian matrices [205]. The inset in the bottom right panel of Fig. 6.4 shows the observed distribution associated with the $1 \times 6$ rectangular matrices. The Goetschy-Stone prediction is drawn in red for reference. The distribution already shows a maximum at intermediate singular values, a key characteristic of the MP distribution.

\subsection{Discussion}

In summary, we have successfully solved a long-standing problem by showing experimental proof of the bimodal behavior of the transmission singular values of scattering systems. This was enabled by having access to all input and output modes of our system. The singular value distributions with a reduced number of modes follow the predictions of Goetschy and Stone [52] with a near-perfect quantitative agreement. This confirms the long-standing hypothesis that the open channels can only be recovered when all modes can experimentally be accessed. Our work fits in a trend of using well-defined photonic systems to investigate scattering physics [159, 210-214]. Our large, low loss and fully tunable processor can be used to study, for example, multi-photon interference effects in disordered systems, universal conductance fluctuations, or Anderson localization [47]. Another future direction could be to use recirculating mesh design, which allows for a more natural correspondence to the physical scattering systems [155]. 


\section{ApPENDix 6}

\section{A Simulation of scattering systems}

The simulation of the 12-mode $S$-matrices follows the model as proposed by Dorokhov, Mello, Pereira and Kumar [49, 50], which models the scattering system into a perfect $1 D$ waveguide short segments. Each segment is shorter than the transport mean free path $l^{*}$ and longer than the wavelength. Adding a new segment can now be described as a perturbative correction [47]. We follow the transfer method of Ko and Inkson [207] for numerical stability.

In our case, the matrices are computed by simulating a one-dimensional 6-mode waveguide with perfectly reflecting boundary conditions. The waveguide is divided into 40 equally sized sections over the length of the waveguide. Each section has a probability of $10 \%$ to have a scatterer placed at a random coordinate inside this waveguide segment. This probability corresponds to the weak scattering regime.

The probability to encounter a scatterer relates to the transport mean free path $l^{*}$. Furthermore, given $l$, the number of segments $N$ determines the average transmission efficiency. In our case, we chose $N=40$ and $\langle T\rangle=0.37$ as this allows us to observe open channels with 200 random instances of these waveguides. Stronger-scattering waveguides, i.e. with more segments and scatterers, have lower average transmission such that an insufficient number of singular values can be sampled to resolve the open channels. The limit of 200 matrices is chosen for experimental convenience.

\section{B Matrix embedding}

Our matrix embedding procedure with the no-gain assumption implies that all singular values of the entire $S$ matrix should be smaller than or equal to 1 . This is achieved by embedding the $6 \times 6$ transmission matrix inside a larger $12 \times 12$ matrix [166]. In this section we describe this procedure.

The $n \times n$ scattering matrix $S$ can always be decomposed by the singular value decomposition: $\operatorname{svd}(S)=U \Sigma V^{*}$. Here, the unitary matrices $U$ and $V^{*}$ describe some basis transformation to the eigenvectors of the matrix. The matrix $\Sigma$ is a diagonal matrix with the singular values and describes the 'weight' of the eigenmode. Ideally, the network is lossless and as a result, $S$ must be unitary. In that case, the diagonal elements of $\Sigma$ are all of the form of $e^{i \theta_{n}}$, with $\theta_{n}$ some phase of the $n^{\text {th }}$ singular value. In case the amplitude of a singular value is $<1$, then the corresponding eigenmode is lossy and if it is $>1$, then it has gain.

In our case, we only have access to a noisy version of the transmission matrix. The noise eludes the observation of the open channels, so it is essential to mitigate the noise on $T$. We achieve this by embedding the transmission matrix $T$ inside a larger, unitary matrix which is constructed using the matrices of the singular value decomposition. This is necessary as it is not possible to impose unitarity. The process of embedding 
the transmission matrix inside a larger, unitary matrix can physically be understood by interpreting loss as a beam splitter where one of its output modes directs the light to an unobserved, inaccessible mode [68]. The exact splitting ratio corresponds directly to the loss in the system. This indicates that the larger unitary matrix should be at least twice the size of the physical system, so that each mode can have at least one loss channel available. Note that gain is nonphysical in our system as there is no additional light source present, besides the injected photons.

The goal now is to first construct a new unitary matrix $S^{\prime}$ that incorporates the coupling to the environment and then to impose the no-gain assumption. For the first step, we construct new matrices $U^{\prime}, \Sigma^{\prime}$ and $V^{\prime}$, which together form $S^{\prime}=U^{\prime} \Sigma^{\prime} V^{\prime *}$, which incorporates the coupling to the environment. Recall that $\operatorname{svd}(T)=U \Sigma V^{*}$.

The new matrix $U^{\prime}$ can be constructed by:

$$
U^{\prime}=\left[\begin{array}{ll}
U & 0 \\
0 & I
\end{array}\right],
$$

where the bottom right of $U^{\prime}$ is filled with an identity matrix for convenience. In principle, any unitary matrix can be used as there is no input or output in any of the unobserved modes anyway. The matrix for $V^{\prime}$ is constructed similarly.

The matrix $\Sigma^{\prime}$ now denotes not just the singular values, but also the coupling to the unobserved modes, i.e., the loss channels. This results in four quadrants, each quadrant is a diagonal matrix. The off-diagonal quadrants denote the coupling to the environment and are constructed such that the energy is conserved in an $L_{2}$ (Euclidian) norm. The new matrix is given by

$$
\Sigma^{\prime}=\left[\begin{array}{c|c}
D_{i} & D_{o} \\
\hline D_{o} & D_{i}
\end{array}\right]
$$

with $D_{i}$ and $D_{o}$ matrices given by:

$$
D_{i}=\left[\begin{array}{cccc}
\cos ^{2} \theta_{1} & 0 & \ldots & 0 \\
0 & \cos ^{2} \theta_{2} & & \vdots \\
\vdots & & \ddots & 0 \\
0 & \ldots & 0 & \cos ^{2} \theta_{n}
\end{array}\right]
$$

and

$$
D_{o}=\left[\begin{array}{cccc}
\sin ^{2} \theta_{1} & 0 & \ldots & 0 \\
0 & \sin ^{2} \theta_{2} & & \vdots \\
\vdots & & \ddots & 0 \\
0 & \ldots & 0 & \sin ^{2} \theta_{n}
\end{array}\right]
$$

In these matrices, $\cos ^{2} \theta_{i}=\tau^{\prime}$, with $\tau^{\prime}$ the singular values after the no-gain restriction. The no-gain restriction entails that no $\tau>1$ as it is nonphysical in our system. Hence the maximal allowed singular value is $\tau=1$, meaning that all singular values of a matrix 
should be rescaled to $\tau^{\prime}=\tau / \max \tau$. Imposing no gain in the transmission values is a sufficient error correction strategy for suppressing experimental noise and retrieving the bimodal distribution without going so far as imposing unitarity on $S$. These renormalized singular values are then used to compute $\Sigma^{\prime}$ in Eq. 6.4. The resulting new singular values are shown in Fig. 6.3b) of the main text. 



\section{An efficient, one-sided device-independent photonic indistinguishability witness}

Quantum photonics has shown substantial potential as a platform for near-term quantum information processing, culminating in the recent demonstrations of a quantum advantage in boson sampling $[11,25]$, i.e., of experiments in which a quantum device outperforms a classical one at a certain computational task. In these experiments $[11,18-21,23,25$, $64,162,171]$, photons are generated by a series of sources and sent through a large-scale linear interferometer, which can be implemented either in static form (typically in bulk optics) or as a tunable interferometer in integrated optics. An advantage arises because it is believed to be classically computationally hard to draw samples from the probability distribution that arises when the output state of the interferometer is measured in the Fock basis $[15,215]$.

These experiments operate at the edges of what is technologically possible, requiring very high-quality optical systems. Low-quality components introduce noise, which may open up a loophole, making the system susceptible to efficient classical simulation [66, $70,75]$. In particular, it is required that the optical system have low loss [59, 67, 68, 216], and that the photons have a high degree of indistinguishability [66], i.e. that the photons are identical in those aspects of their wave function not perturbed by the interferometer, such as wavelength, polarization, and temporal structure.

Indistinguishability is central in linear-optical information processing, both in the boson sampling paradigm and for universal linear-optical quantum computation [15, 42, 217], because it enables the creation of entanglement between non-interacting particles. This entanglement arises due to the requirement that a quantum state comprised of multiple indistinguishable bosons must have an overall symmetric wave function. The Hong-OuMandel effect [133] is the archetypical example of this process, and the quantum advantage experiments of $[11,25]$ can be thought of as multiphoton, multimode generalizations of this effect. Accurate measurements of the photon indistinguishability are therefore central to the discussion surrounding the classical simulability of recent quantum advantage experiments [54-56, 153, 173, 218-224]. This must be done in an experimental configuration as close to that in which a quantum advantage is obtained, and ideally on all photons simultaneously.

To address this problem, Brod et al. [56] introduced the notion of an indistinguishability witness. Analogous to entanglement witnesses [225], for a given linear network, an indistinguishability witness is an observable $\hat{C}(U)$ whose expected value $C(U)$ has some upper bound $C_{C}(U)$ for distinguishable particles, but whose value for indistinguishable particles $C_{q}(U)$ exceeds that bound. When we measure $C$ in an experiment and find a value that exceeds $C_{c}(U)$, we can therefore infer that the photons used are indistinguishable. 
However, Shchesnovich [226] pointed out that such witnesses are susceptible to implementation loopholes: for a specific combination of linear network $U$ and a witness $C$, it is possible that there will be some other linear network $U^{\prime}$ which has a larger value of $C_{c}\left(U^{\prime}\right)>C_{c}(U)$. If this is the case, then if the linear interferometer is set to $U^{\prime}$ instead of to $U$, indistinguishability would be falsely certified if $C_{c}\left(U^{\prime}\right)>C_{q}(U)$. This is analogous to the situation in entanglement witnesses, which can be compromised if an adversarial party takes over a trusted detection apparatus [227] or if the control over the experimental apparatus is imperfect [46].

For the analogous problem of certifying other quantum properties such as entanglement or randomness, a wide array of approaches have appeared which relax the assumptions made on the measurement apparatus. The most dramatic of these are fully deviceindependent protocols [228, 229], which rely on bell nonlocality [230], to provide a highly rigorous level of certification but which are extremely experimentally challenging $[231,232]$. The plethora of intermediate methods that trade practicality for additional assumptions and characterization are known as semi-device-independent [233-242].

In this chapter, we present a robust solution for witnessing indistinguishability, namely to maximize $C_{C}(U)$ over $U$, i.e. to find the largest achievable value of $C$ over all linear networks, and choose that as the threshold value. In this sense, the witness is semidevice independent: if the matrix settings are corrupted, the experiment will simply fail to certify indistinguishability, but it will never incorrectly certify a set of distinguishable photons as being indistinguishable. We show that this witness, which is based on twomode correlators, can be efficiently measured in an experiment. We implement this witness in an experiment using a tunable integrated photonic interferometer [46] and a multiphoton source, and use it to certify the indistinguishability of three photons. Furthermore, we show that the degree to which this witness violates its classical threshold can be used to lower bound the degree of indistinguishability between the photons. We have therefore introduced an efficient method of unambiguously certifying multiphoton indistinguishability.

\subsection{Indistinguishability witness}

The basis of our method are the Ursell functions [243], which are referred to as $n$ mode correlators in a quantum optical context. These functions measure the degree of excess correlation among a subset of the optical modes of the interferometer. The use of these functions in this context was pioneered by Walschaers et al. [55], where the two-mode correlation function was used to perform a statistical certification of photon indistinguishability. Our goal is to show that by looking at the extremal values of these correlators, one can transform this probabilistic certification method into a deterministic one.

The two-mode correlators of [55] are given by:

$$
C_{i, j}=\left\langle\hat{n}_{i} \hat{n}_{j}\right\rangle-\left\langle\hat{n}_{i}\right\rangle\left\langle\hat{n}_{j}\right\rangle,
$$




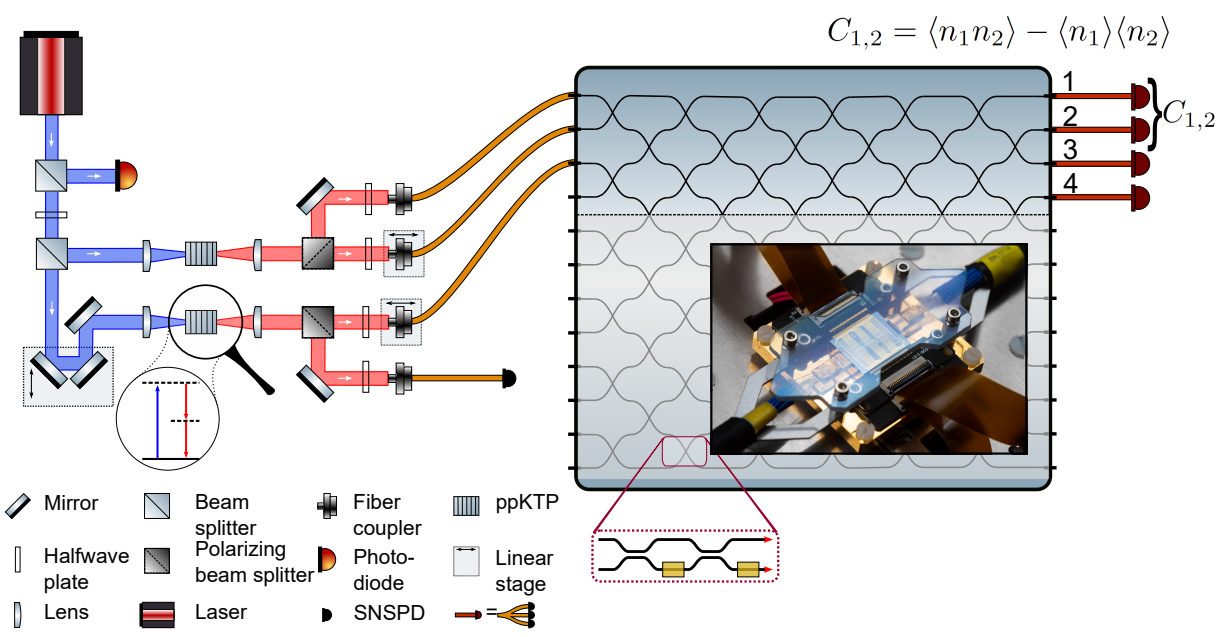

Figure 7.1 Experimental setup. A $775 \mathrm{~nm}$ pulsed laser (blue) pumps the two periodically poled KPT (ppKTP) spontaneous parametric down-conversion sources. Each crystal probabilistically generates a pair of two photons with orthogonal polarization (red). One of these four photons is used as a herald and the remaining three are injected in the first three modes of our $12 \times 12$ integrated universal programmable processor. The processor output is sent to a small fiber-beamsplitter and SNSPDs acting as pseudo-number counting detectors. In the processor, the top 4 modes are used to implement the transmission matrices. The zoom-in shows a unit cell of a MachZehnder interferometer that implements one of the programmable beam-splitters. The inset shows a photograph of the fiber-connected integrated optical chip used for this experiment.

where $\langle$.$\rangle denotes the expected value, and \hat{n}_{i}$ is the photon number operator acting on optical mode $i$. Considering the case of a linear optical circuit given by a transformation matrix $U$, with one photon injected at each of the first $n$ input ports, this equation can be evaluated by noting that $\left\langle\hat{n}_{i}\right\rangle$ follows from single-photon transmission probabilities:

$$
\left\langle\hat{n}_{i}\right\rangle=\sum_{k=1}^{n}\left|U_{k, i}\right|^{2}
$$

Furthermore, $\left\langle\hat{n}_{i} \hat{n}_{j}\right\rangle$ can be determined by summing all combinations that result in photons in output modes $i$ and $j$ and the partial distinguishability between the photons [244]:

$$
\left\langle\hat{n}_{i} \hat{n}_{j}\right\rangle=\sum_{k \neq l=1}^{n}\left(\left|U_{i, k}\right|^{2}\left|U_{j, l}\right|^{2}+\left.\left\langle\psi_{k} \mid \psi_{l}\right\rangle\right|^{2} U_{i, k} U_{j, l} U_{i, l}^{*} U_{j, k}^{*}\right) .
$$


Combining the above equations leads to the following expression for the correlator:

$$
\begin{aligned}
C_{i, j}= & -\sum_{k=1}^{n}\left|U_{i, k}\right|^{2}\left|U_{j, k}\right|^{2} \\
& +\left.\sum_{k \neq l=1}^{n}||\left\langle\psi_{k} \mid \psi_{l}\right\rangle\right|^{2} U_{i, k} U_{j, l} U_{i, l}^{*} U_{j, k}^{*}
\end{aligned}
$$

where $U$ is the transfer matrix of the linear optical system, and $x^{2}=\left.\left\langle\psi_{k} \mid \psi_{l}\right\rangle\right|^{2}$ is a quantity measuring the degree of indistinguishability in terms of the internal wave functions of the photons, with $x=0$ corresponding to distinguishable photons and $x=1$ corresponding to completely indistinguishable ones. Note that the first term in eq. 7.4 corresponds with classical transmission of light, since it is not affected by the degree of distinguishability, whereas the second term describes the quantum interference. Without loss of generality, we restrict ourselves to the first two modes in the network, hence we drop these indices in the notation. We will now show that this two-point correlator can be used as a semidevice-independent witness.

The correlator can function as a semi-device-independent distinguishability witness if and only if its value to a lower bound on the degree of indistinguishability, regardless of the implemented network. We must therefore maximize eq. 2 for distinguishable photons, i.e. when the second term is zero. It is straightforward to see that a matrix that obeys the condition $\forall k \leq n,\left(U_{i, k}=0\right) \vee\left(U_{j, k}=0\right)$ maximizes $C$ for distinguishable particles at $C_{c}=0$. An example of such a matrix is the identity matrix. The fact that all terms in the first sum in eq. 2 are negative implies that this solution is optimal. This proves that regardless of the matrix implemented, $C_{c}(U) \leq 0$. This result has a simple interpretation: in the absence of entanglement, the only correlation allowed between particles is via the total photon number, which is conserved. Therefore, if a particle is observed in a particular mode, it decreases the probability of observing any particle in another mode.

Next, we must find a matrix which has $C_{q}>0$. In principle, any matrix which obeys this condition suffices. However, we believe we have found the optimal matrix based on symmetry arguments: even if we do not assume all pairs of photons are equally indistinguishable, we still do not know in advance which pairs will be more or less indistinguishable, and therefore all modes containing a photon must be treated on the same footing. Furthermore, we observe that the second term in eq. 2 is maximized if all elements are in phase. Our conjectured solution, which is supported by numerical evidence $^{1}$, is a matrix of size $N=n+1$, with a highly symmetric form:

$$
U_{\max }=\left(\begin{array}{cccc}
\frac{1}{\sqrt{2 n}} & \cdots & \frac{1}{\sqrt{2 n}} & \frac{1}{\sqrt{2}} \\
\frac{1}{\sqrt{2 n}} & \cdots & \frac{1}{\sqrt{2 n}} & -\frac{1}{\sqrt{2}} \\
* & * & * & * \\
* & * & * & *
\end{array}\right),
$$

where a $*$ denotes elements that do not enter in the correlator. Note also that since we are only interested in the correlator between the first and second mode, we need only specify

1 See Appendix 7.B 
the first two rows of the matrix; if these are orthogonal (which is enforced by the $n+1$ st column, the Gram-Schmidt process guarantees that we can always complete the matrix in an orthogonal fashion.

The correlator for this matrix as a function of the indistinguishability $x$ follows trivially from eq. 7.5:

$$
C(x)=\frac{x^{2}}{4}-\frac{1+x^{2}}{4 n}
$$

where $\sum_{k \neq l=1}^{n}\left|\left\langle\psi_{k} \mid \psi_{l}\right\rangle\right|^{2}=n(n-1) x^{2}$ is used. The threshold is $C_{q}=\frac{1}{4}-\frac{1}{2 n}$, which is larger than $C_{c}=0$ for all $n$ greater than 2; therefore, using this method, we can certify indistinguishability between 3 or more photons.

To see that this witness is efficient, observe that the probability that the average photon flux on either of the two modes of interest is $\frac{1}{2}$, irrespective of the number of photons. This means that the probability that a given a given run of the experiment contributes to the measurement statistics of $C$ is $\frac{3}{4}$. This quantity is independent of $n$ and $m$. Finally, we note that the precision required to certify indistinguishability between $n$ and $n+1$ photons grows quadratically with $n$. These facts together prove that the scheme is efficient in the number of measurements required.

It is also intuitive that when drawing matrices uniformly according to the Haar measure, the probability of being close to $U_{\text {ideal }}$ decreases with matrix size, a fact which is supported by numerical simulations. This result shows that control over the interferometer is required to implement this witness; the probability of it arising naturally is small.

Finally, we discuss the limits of this witness: it cannot certify indistinguishability if there are only two photons present, and it cannot certify indistinguishability if the average value $x^{2} \leq 0.5$

\subsection{Setup}

To measure this semi-device-independent entanglement witness, we carry out an experiment using a tuneable linear optical interferometer, a photon source and a bank of single-photon detectors.

Fig. 7.1 shows our experimental setup. The optical network is a 12-mode quantum photonic processor [46] implemented in silicon nitride waveguides [176]. The chip consists of a grid of unit cells, as shown in Fig. 7.1 [177]. Each cell consists of a Mach-Zehnder interferometer with two $50: 50$ static beam splitters and two tunable phase shifters. Together, these elements can implement any arbitrary pairwise mode interaction. The network is fully tunable using the thermo-optical effect. The total optical transmission through the chip varies between $54 \%$ to $60 \%$, depending on the particular combination of input and output modes. In our experiment, only the top four modes of the chip are used.

The photon source consists of two $2 \mathrm{~mm}$ ppKTP crystals in a Type-II degenerate configuration. The crystals are pumped with $60 \mathrm{~mW}$ of $120 \mathrm{fs}$ pulsed light at $775 \mathrm{~nm}$. Each crystal generates a pair of photons probabilistically at $1550 \mathrm{~nm}$ [245]. We herald one photon and postselect on fourfold coincidences to herald the input state $\left|\psi_{\text {in }}\right\rangle=|1110\rangle$. 
The photons are $93 \%$ indistinguishable when they originate from the same crystal (signalidler), and 78\% indistinguishable when they are from different crystals (signal-signal). This corresponds to an average three-photon indistinguishability of $x^{2}=0.81$. The average fourfold coincidence rate is $250 \mathrm{~Hz}$ without the chip.

The photons are detected using 12 superconducting single-photon detectors [246, 247]. Each output mode of the chip directs the photons towards three detectors, which are placed in parallel. This effectively functions as a pseudo-photon-number-resolving detector [248]. The relative detection efficiencies and splitting ratios are calibrated by sequentially sending photons from input mode 1 to all 4 output modes and comparing the relative count rates. The detectors are read out using standard correlation electronics. See Appendix 8.C for more details on the quasi-photon-number-resolving detectors and characterization of relative detection efficiencies.

\subsection{Results}

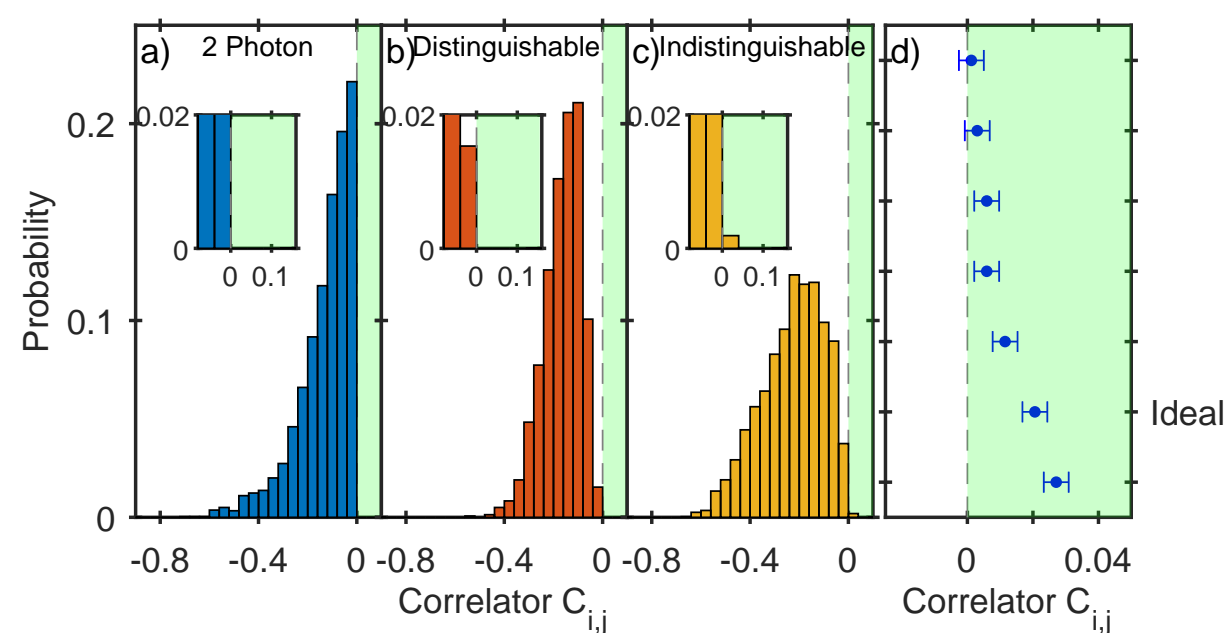

Figure 7.2 Experimental results. The distribution of the two-point correlator is never larger than 0 for a) two identical photons and b) three distinguishable photons. For three indistinguishable photons (c), several instances of positive correlators are observed. The insets show a zoom-in at $C=0$. All positive correlators and the corresponding error bar (1 standard deviation) are shown in an ordered fashion in d).

We begin with examining the statistical distributions of the two-point correlator for Haar-random matrices. We implement 500 randomly chosen $4 \times 4$ Haar-random matrices. For each implemented matrix, all $\left(\begin{array}{l}4 \\ 2\end{array}\right)=6$ two-mode correlators are measured for 3 minutes 2 .

2 In data analysis, it became apparent that due to a communication error, that the second phase shifter of the 


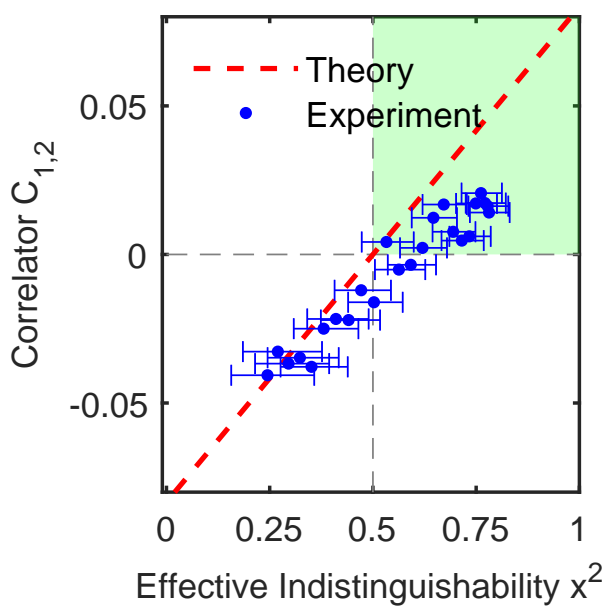

Figure 7.3 Ideal matrix. The correlator for the ideal matrix with imperfect matrix control. The red dashed line indicates the maximal correlator possible with the ideal matrix. The inset shows how the effective indistinguishability was varied by changing temporal overlap of the third photon with respect to the other two photons before injecting them into the chip.

Figure 7.2a shows the experimentally observed distributions when two indistinguishable photons are injected into the first two modes of the interferometer. All correlators are negative, as expected, and the distribution follows the expected shape, peaking at the cutoff of $C_{c}=0$ [55]. The inset shows a zoom-in at the correlators around this cutoff. The overall shape of the distribution is as expected for two photons.

In Fig. 7.2b, the correlator distribution for 3 fully distinguishable photons is shown. This distribution is similar to that of two photons, but has shifted somewhat towards negative values, As expected, no positive correlator $C>0$ is observed in this distribution.

Finally, Fig. 7.2c) shows positive two-point correlators when three partially indistinguishable photons are injected into the network. This is the only case where matrices with $C>0$ are observed, certifying indistinguishability.

Fig. 7.2d shows a bar chart of all cases where we observe positive correlators in the experiment with three indistinguishable photons. The label 'ideal' refers to the matrix given in eq. 7.5. The error bars indicate the standard deviation assuming Poissonian noise. Multiple data points, including the one corresponding to $U_{\max }$ are statistically significantly above the threshold value $C_{c}=0$. The largest observed correlator is $C=0.027>C_{c}$, exceeding the threshold by $7 \sigma$. Therefore, we can certify indistinguishability between these three photons.

We now turn out attention exclusively to the matrix $U_{\max }$. To test the robustness of our witness, we decrease the degree of distinguishability between one of the three photons

top-left unit cell was not actuated on during measurements. This does not significantly affect the results of the Haar measurements. 
and the other two by varying the time of arrival in the interferometer.

Figure 7.3 shows that the correlator increases monotonically when the temporal overlap of the photons is increased by varying the time of arrival of the third photon in the interferometer. The correlator reaches a maximal value $C_{i, j}=0.0206 \pm 0.0038$. As expected, no data points are observed above the ideal red dashed line when $x^{2}>0.5$, which emphasizes the optimal nature of our matrix.

The maximal observed correlator is lower than the expected $C_{\max }=0.052$ for the effective indistinguishability $x^{2}=0.81$, and the rate at which $C_{i, j}$ increases with increasing indistinguishability is also lower than expected. Both the reduced slope and correlator can be understood by imperfect chip control. Implementing the ideal matrix with a fidelity of $98 \%$ already reduces the average correlator to $C_{i, j}=0.024 \pm 0.007$ for partial indistinguishable photons. Fully indistinguishable photons have a maximal $C_{\max }=$ $0.060 \pm 0.008$ in case $98 \%$ implementing fidelity ${ }^{3}$. This behavior is typical for a semidevice independent witness: imperfect control over the system reduces the parameter regime in which quantumness can be certified, but it never opens up a loophole where quantumness will be claimed if it is not there.

\subsection{Outlook}

We leave it as an open question to what extent the device independence assumptions introduced here can be further relaxed, for example by removing the requirement that the photons are in single-photon Fock states at the input.

We note that it is straightforward to modify our scheme into an adversarial setting where one party provides photons that are claimed to be indistinguishable, and the other party either tests this fact or uses those photons to generate a sample from a given boson sampler.

\subsection{Conclusion}

In conclusion, we present an experimental demonstration of a semi-device-independent indistinguishability witness for multi-photon interference. The witness requires only the measurement of a single two-point correlator behind two fixed output modes of a tunable optical network. This correlator is shown to be maximal for the so-called ideal matrix. Consequently, any imperfect implementation of this ideal matrix will inevitably reduce the correlator, demonstrating its suitability as a semi-device independent witness. Similar to the entanglement witness, this result can function as a diagnostic tool in experiments. For example, this work opens the way to robust, efficient in-situ characterization of boson sampling experiments and experiments derived from boson sampling.

3 See Appendix 7.A for more details on the error analysis. 


\section{ApPENDix 7}

\section{A Noisy ideal matrix}

A Monte-Carlo simulation has been performed to investigate the effect of limited matrix implementation on the chip in the experiment. A total of 15000 instances of the ideal matrix with random complex Gaussian noise have been generated. Each noisy matrix has been unitarized by a polar decomposition and then the fidelity has been calculated by:

$$
F=\frac{1}{N} \operatorname{Tr}\left(\left|U_{\mathrm{t}}^{\dagger} U_{\mathrm{exp}}\right|\right)
$$

Figure 7.4 shows how the fidelity affects the $C_{1,2}$ correlator of the ideal matrix. Figure 7.4a) shows different outcomes for $C_{1,2}$ for three fully distinguishable photons. Figure 7.4b) shows the same for photons with partial distinguishability as observed in our experiment and finally 7.4c) shows the correlators for three indistinguishable photons. In all cases, none of the observed correlators are above the maximal allowed value as indicated by the red dashed line.

This integrated optical network is reported to have an average amplitude fidelities of about $90 \%$ for Haar-random matrices of the full $12 \times 12$ network [46]. In our experiment, only a part of the processor is used and the fidelities are close to $99 \%$. For such fidelity, the average $C_{1,2}=0.03 \pm 0.003$ for the partial distinguishable photons. The propagation of partial distinguishability through the network has been calculated with the tensor method of Tichy et al. [153].

\section{B Monte-Carlo search for the ideal matrix}

A second Monte-Carlo simulation has been performed to explicitly search for a $4 \times 4$ unitary matrix that can yield higher correlators than provided by the ideal matrix as given in eq. 7.6. To this end, the average photon indistinguishability is varied from $x=0$ to 1 for both $n=2$ and $n=3$ photons. All photons are assumed to be equally indistinguishable from all other photons. A total of $10^{7}$ Haar random matrices are generated for each average photon indistinguishability. For each average indistinguishability, the matrix that gives rise to the highest correlator $C_{i, j}$ is saved.

The maximum correlators found for each average indistinguishability are plotted in Fig. 7.5. The correlators corresponding to the ideal matrix are shown in a dashed black line going diagonally through the plot. The maximum correlators for each average indistinguishability up to $x^{2}=0.5$ is (just) under 0 for both $n=2$ and $n=3$ photons. After $x^{2}=0.5$, the correlator for two photons remains smaller than 0 , the highest observed correlator is $C=-1.6 * 10^{-4}$. In the case of three photons, the correlator increases after $x^{2}=0.5$ and follows the correlators corresponding to $U_{\mathrm{ideal}}$. At no point, the correlators found by the Monte-Carlo simulation exceed the correlators associated with $U_{\text {ideal }}$. This strengthens the notion that the ideal matrix is indeed optimal. 


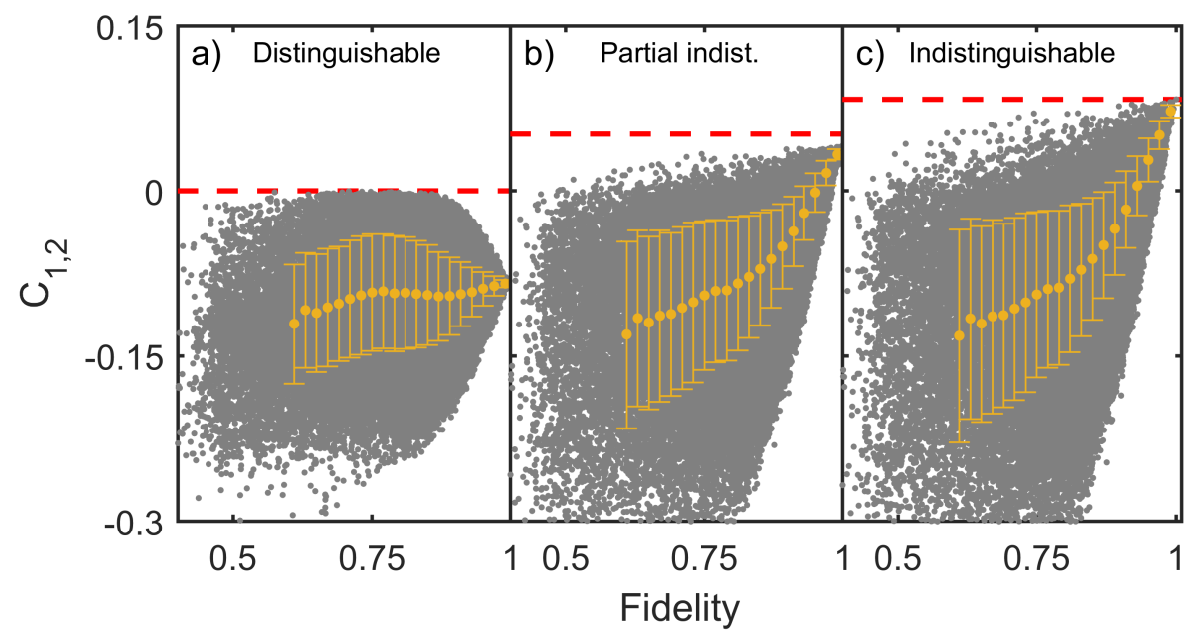

Figure 7.4 A Monte-Carlo simulation of imperfect implementation of the ideal matrix for a) distinguishable, b) partial indistinguishable and c) fully indistinguishable photons. The simulated partial indistinguishability is set to what is observed in our lab. Each panel shows how the two-point correlator improves with the fidelity of the matrix implementation. The red dashed lines indicate the maximal allowed value for $C_{1,2}$, the grey dots are individual results and the yellow error bars indicate the mean and standard deviation for a selected number of matrix fidelities.

Figure 7.5 still clearly has a discrepancy in the range for $x^{2} \leq 0.5$. The correlators found by the Monte-Carlo simulation are substantially higher than the ones from the ideal matrix. Upon closer inspection, it seems that the optimal matrix corresponding with distinguishable photons is the identity matrix. It is easy to show that the maximum correlator with the identity matrix is indeed 0 . Hence, the applicability of our ideal matrix method is limited to the case where the photons have an average indistinguishability of at least $x^{2}=0.5$. Nonetheless, this limitation will not result in misleadingly high correlators and the protocol is still robust. 


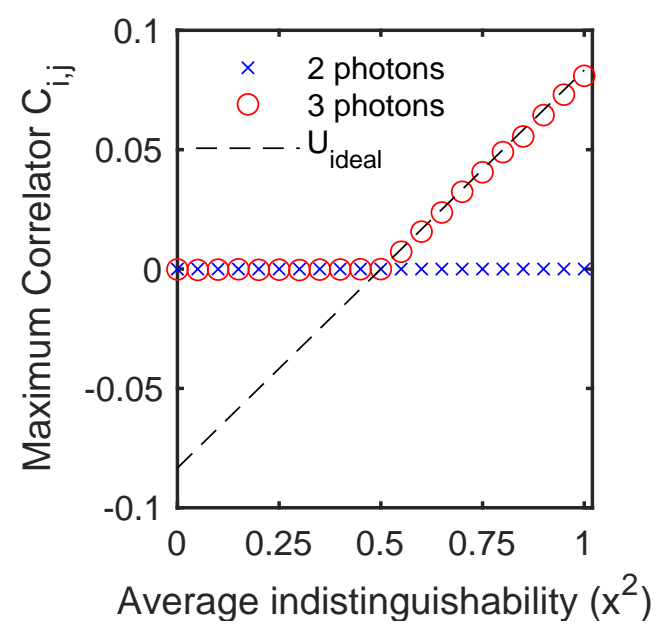

Figure 7.5 A Monte-Carlo search for the ideal matrix. The highest correlators from a total of $10^{7}$ random matrices are plotted for each partial indistinguishability. None of the data points for $n=2$ and $n=3$ photons exceed $U_{\text {ideal }}$. 



\section{Quantum}

\section{photo-thermodynamics on a programmable photonic quantum processor}

One of the long-standing puzzles of theoretical physics is how notions of statistical physics and of basic quantum mechanics precisely fit together [249]. Statistical mechanics speaks in probabilistic terms of stationary ensembles that maximize entropy under external constraints. Elementary quantum mechanics, in contrast, describes the deterministic evolution of the quantum state of closed systems under Hamiltonians that specify the physical situation at hand. It has become clear that these seemingly contradictory premises can be resolved by accepting that global unitary dynamics and local relaxation are perfectly compatible; after all, all that needs to be explained is how expectation values of local observables take values as if the entire system had globally relaxed. Theoretical work in the last decade has much contributed to a better understanding of equilibration and thermalization in closed quantum many-body systems [57, 250-252]. This work is particularly clear in situations in which the initial state holds a memory of a non-Gaussian initial state, but where the actual Hamiltonian dynamics is governed by a non-interacting quadratic bosonic Hamiltonian. Here, indeed, physical systems are expected to "Gaussify" in time, i.e., to converge seemingly to Gaussian states that would maximize the entropy given all second moments of the state [253-255]. For local Hamiltonian dynamics, such behavior can be proven to occur in all rigor, constituting an example of a situation in which convergence can be shown to a so-called generalized Gibbs ensemble [253, 256-259], i.e. a thermal ensemble under further constants of motion.

Notwithstanding this comparably clear situation from a theoretical perspective, experimental evidence is largely lacking [58, 260-266]. This is primarily due to the fact that it is challenging to create sufficiently isolated experimental systems to rule out that the observed relaxation is not due to quantum information leaking to the environment.

In this chapter, we report experimental results that show reversible Gaussification under experimentally well-controlled conditions. The setting in which we achieve this is integrated photonics (see Fig. 8.2). We use the high degree of control available in optical systems both to implement the desired optical transformation with high accuracy, and to implement a set of verification measurements that certify that the observed relaxation is due to the internal dynamics of our multimode quantum state and not due to interaction with the environment.

Boson sampling [15] experiments and their Gaussian analogs [32] already show that photonic devices bear the potential of performing non-universal quantum computations beyond classical means [25]. Here, we contribute to going a substantial step further and 


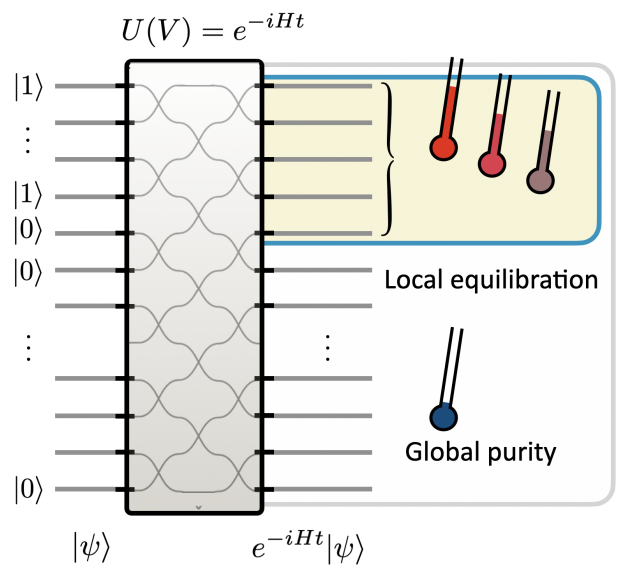

Figure 8.1 Photonic simulation of quantum equilibration. A closed, many-body quantum system, initialized in a product state and undergoing unitary evolution generated by a Hamiltonian, necessarily remains in a pure state. However, local observables may exhibit a generalized thermalization. Entanglement builds up between sub-systems until after some time $t_{\text {eq }}$, each sub-system appears to have approximately relaxed into a maximum entropy state. The paradigmatic case of a non-Gaussian bosonic state evolving under a quadratic Hamiltonian can be probed via a photonic simulation platform. A fully programmable linear optical chip can provide 'snapshots' of the local and global system dynamics for arbitrary times and interaction ranges by implementing the appropriate unitary $U(V)=e^{-i H t}$ with $V \in U(m)$ for $m$ modes.

investigate to what extent the newly found levels of control can be exploited in notions of photonic quantum simulation [160, 267-270]. These steps are urgently required in the development of photonic quantum technologies, to uplift systems set up from primarily paradigmatic devices to ones that have a clear operational purpose. To contribute to the vision of firmly placing optical circuits in the realm of quantum technologies, we contribute methods of efficient certification [271], valuable in their own right. Here we develop a flexible and tuneable platform for photonic quantum simulation allowing for simulating processes of equilibration.

\subsection{Local equilibration and thermalization}

In any setting governed by closed-system Hamiltonian dynamics, equilibration can only happen locally for local observables, since the global entropy must be preserved in time. In the setting considered, the global system is a multimode linear-optical system initially prepared in a highly non-Gaussian state $\rho$ on $m$ bosonic degrees of freedom, namely $|\psi\rangle\langle\psi|$ with $|\psi\rangle=|1, \ldots, 1,0, \ldots, 0\rangle$ of $n=3$ single photons in $m=4$ optical modes. The bosonic modes are associated with annihilation operators $b_{1}, \ldots, b_{m}$. The subsequent integrated linear optical circuit is given by a unitary $V \in U(m)$ that linearly transforms the bosonic modes. In state space such linear optical circuits are reflected by $\rho \mapsto$ 
$\sigma:=U(V) \rho U(V)^{\dagger}$, where $U(V)$ is the physical implementation of the passive mode transformation $V^{1}$. Finally, the output distribution is measured in the Fock basis using quasi-photon-number-resolving detectors, giving measurements of the form $\mu \mapsto P(\mu)$ with

$$
P(\mu)=\left\langle n_{1}, \ldots, n_{m}\left|U(V) \rho U(V)^{\dagger}\right| n_{1}, \ldots, n_{m}\right\rangle,
$$

where $\mu=\left(n_{1}, \ldots, n_{m}\right)$ is a given pattern of detection events.

For our purpose of showing local equilibration, we interpret the evolution $U(V)=$ $e^{-i H t}$ as a fictitious evolution under a Hamiltonian $H$ for time $t>0$, which distributes information. In the linear optical system at hand, we will implement two Hamiltonians, a quadratic bosonic translationally invariant 'hopping' Hamiltonian, resembling the noninteracting limit of a Bose-Hubbard Hamiltonian, and a Haar random transformation $V \in U(m)$ corresponding to a Hamiltonian with random long-range interactions.

As the fictitious time $t$ gets larger, increasingly longer-ranged entanglement builds up. This means that the expected moments of the local photon number $\hat{n}_{j}:=b_{j}^{\dagger} b_{j}$ of each of the output modes labeled $j=1, \ldots, m$ of the state $\sigma$ will increasingly, in the depth of the circuit, equilibrate and lead to a distribution that resembles that of a (generalized) Gibbs ensemble. In other words, as seen in Fig. 1, one encounters local equilibration in that the reduced quantum states of a subset of the modes, or individual modes, equilibrate and take thermal-like values.

To be specific, here, the initial state is a product state (and hence has obviously shortranged correlations) - albeit not being translationally invariant - and the bosonic quadratic Hamiltonian will on the one hand be translationally invariant before it undergoes a time evolution generated by $U(V)=e^{-i H t}$ (or the Haar-random $V \in U(m)$ ). The Gibbs or canonical state reflecting thermal equilibrium is given by $\omega=e^{-\beta H} / \operatorname{tr}\left(e^{-\beta H}\right)$ for a suitable inverse temperature $\beta>0$ that is set by the energy density. In data shown later, the local photon numbers are being compared with that of a Gibbs state. For noninteracting bosonic systems, local equilibration for subsystems consisting of several modes is expected to happen to a generalized Gibbs state: This is particularly transparent in our situation where $H$ is a hopping Hamiltonian which is translationally invariant. Defining the momentum space occupation numbers as

$$
\hat{N}_{k}:=\frac{1}{m} \sum_{x, y=1}^{m} e^{2 \pi i k(y-x) / m} b_{x}^{\dagger} b_{y}
$$

one finds that the generalized Gibbs ensemble is then given by the maximum-entropy state $\omega$ given by

$$
\omega=\operatorname{argmax}\left\{S(\eta): \operatorname{tr}\left(\eta \hat{N}_{k}\right)=\left\langle\psi\left|\hat{N}_{k}\right| \psi\right\rangle \text { for all } k\right\},
$$

associated with an inverse temperature per momentum mode. For an infinite system, convergence to such a state is guaranteed [253-255]. For finite systems, it has been rigorously settled in what sense the state is approximated by such a generalized Gibbs ensemble [253, 256-259] before recurrences set in. For the Haar-random unitaries, we

$1 \quad V \mapsto U(V)$ is referred to the metaplectic representation in technical terms. 
still find Gaussification in expectation, creating an interesting state of affairs, as here the theoretical underpinning is less clear.

For subsystems consisting of a single bosonic mode only, canonical or Gibbs states as well as generalized Gibbs ensembles both give rise to identical photon number distributions reflecting Gaussian states: The state 'Gaussifies' in time. The situation at hand is particularly simple in the situation where the expectation value of the photon number is the same for each of the $m$ output modes. Then for a Gaussian state, the probability of observing $k$ photons reduces to

$$
p(k)=\frac{D^{k}}{(D+1)^{k+1}}\left\{1+O\left(\frac{1}{m}\right)\right\},
$$

where $D=n / m$ is the photon density per mode -see Appendix 8.B for the derivation of this formula. In the experiment, system sizes are relatively small, and it is interesting to see how strong signatures of local equilibration and Gaussification are encountered.

Interestingly, generalized Gibbs ensembles are still not quite thermal or canonical Gibbs states, which would be maximum-entropy states given the expectation value of the energy, but a generalization of that state, due to the non-interacting nature of the Hamiltonian. For example, in full non-equilibrium dynamics under large-scale interacting Bose-Hubbard Hamiltonians (as can be probed with cold atoms in optical lattices [262]) one expects an apparent relaxation to a Gibbs state. In contrast, a generalized Gibbs ensemble maximizes the von-Neumann entropy under the constraint of the energy expectation and the momentum space occupation numbers which are preserved under the non-interacting translationally invariant evolution $t \mapsto e^{-i H t}$. Therefore, one can say that each of the momentum modes is then associated with its own temperature, as sketched in Fig. 8.1, and the system 'thermalizes' up to the constraints of the momentum space occupation numbers being preserved.

\subsection{Certification}

A crucial aspect of our results is demonstrating that the resulting thermalization is not due to entanglement with the environment (i.e. decoherence), but rather to entanglement between the photons. We make use of the intuition that entanglement between the photons is reversible whereas decoherence is not (at least in this setting), to introduce a certification protocol for our thermalization experiment.

General idea. Our certification method is a fidelity witness [272] that certifies the fidelity, $F\left(\rho,\left|\psi_{t}\right\rangle\right)=\left|\left\langle\psi_{t}|\rho| \psi_{t}\right\rangle\right|$, between the experimental state, $\rho$, and a pure target state, $\left|\psi_{t}\right\rangle=U|\psi\rangle$, for an arbitrary linear optical unitary and single-photon input states. The procedure requires a well-calibrated, programmable measurement unitary and number resolving (but not spectral-mode resolving detectors). It consists of two settings for the measurement unitary: the inverse of the target unitary and a the inverse followed by a Fourier transform. The constant number of measurement settings and polynomial classical computation resources required mean the procedure is efficiently scalable to 
arbitrary system sizes. Here we give a brief outline of the certification protocol, which is explained further in the Appendix 8.C.

Fidelity estimation. Here, we consider three photons (possibly in distinct temporal modes) in four spatial modes. The target pure state is defined as a target unitary acting on an initial state with one photon in each of the first three spatial modes and all photons in the same temporal mode.

Given full mode resolution and a well-calibrated, programmable measurement unitary, $F\left(\rho,\left|\psi_{t}\right\rangle\right)$ can be directly estimated by measuring the photon number of the state $U^{\dagger} \rho U$ [272]. Whilst the requisite linear optical measurement unitary be efficiently characterized in detail, offline and using only classical light [273], in our architecture, the photon detectors cannot perfectly resolve temporal and spectral degrees of freedom. Neglecting this and naively carrying out the above procedure could result in certifying a large fidelity even with photons in distinct temporal modes, i.e. distinguishable states.

To rule this out we employ two additional measurement settings: 1) we implement $U^{\dagger}$ and count photons 2 ) we implement $U^{\dagger}$ followed by a Fourier transformation and count photons. From the first setting, we upper bound the probability, $p_{1}$, of seeing one photon in each of the first three spatial modes and no photon in the fourth. From the second, we upper bound $p_{2}$, the overlap probability of $\rho$ with the distinguishable subspace. This is done by monitoring the fraction of observed interference patterns that would be forbidden for truly indistinguishable photons following a Fourier transform [274-276].

In this way we arrive at a fidelity bound of the form

$$
F \geq p_{1}-\frac{9}{4} p_{2}-\delta(\epsilon)
$$

which holds with a probability of at least $\epsilon=\epsilon_{1} \epsilon_{2}$ where the probabilities arise from estimating $p_{1}$ and $p_{2}$ from the observed photon counting statistics and $\delta$ is the corresponding statistical penalty. For a full derivation see 8.A.

Entanglement witness. If one is merely interested in establishing the presence of entanglement in the system, one can derive a simple entanglement witness $\mathcal{W}$ from the estimated fidelity. We use the following definition of an entanglement witness [277]

$$
\mathcal{W}=\lambda_{\max }^{2} \mathbb{I}-\left|\Psi_{t}\right\rangle\left\langle\Psi_{t}\right|
$$

where $\lambda_{\max }^{2}$ is the maximal Schmidt coefficient in the decomposition of $\left|\Psi_{t}\right\rangle$, whose classical computation is not scalable, but feasible in our case.

\subsection{Integrated photonic platform}

We use an integrated quantum photonics architecture to serve as our experimental platform (see Fig. 8.2). Integrated quantum photonics constitutes a platform for non-universal quantum simulation based on bosonic interaction between indistinguishable photons [20, $156,160,161,171,278]$. In integrated quantum photonics, quantum states of light are 


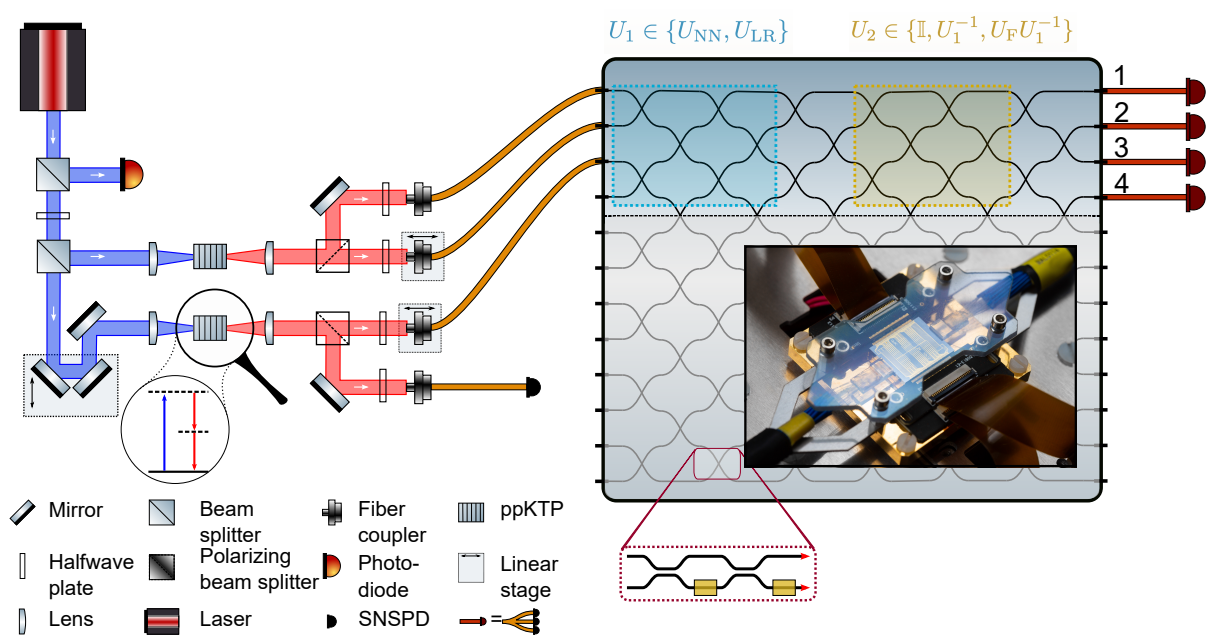

Figure 8.2 Overview of the setup. On the left the double spontaneous parametric downconversion $(S P D C)$ source is sketched, in which blue pump photons are spontaneously split in two red photon pairs. One of these four photons is used as a herald and the other three are injected in the first three modes of our $12 \times 12$ integrated universal programmable processor. The processor output is sent to small fiber-beam-splitter networks and SNSPDs acting as pseudo-number counting detectors. In the processor, we program the unitary $\mathrm{U}_{1}$ used to simulate the temporal dynamics (the blue block). In addition, we can program a second unitary $\mathrm{U}_{2}$ for the verification process (the yellow block). The zoom-in shows a Mach-Zehnder interferometer that implements one of the programmable beam-splitters. The inset shows a photograph of the fiber-connected integrated optical chip used for the experiment. 
fed into a large-scale tunable interferometer and measured by single-photon-sensitive detectors.

Our interferometer is realized in silicon nitride waveguides [46, 176], and has an overall size of $N=12$ modes and an optical transmission of $2.2-2.7 \mathrm{~dB}$, i.e. $54 \%-60 \%$ depending on the input channel. Reconfigurability of the interferometer is achieved by a suitable arrangement of unit cells consisting of pairwise mode interactions realized as tunable Mach-Zehnder interferometers [177]. Each unit cell of the interferometer is tunable by the thermo-optic effect. The average amplitude fidelity $F=\frac{1}{N} \operatorname{Tr}\left(\left|U_{\text {set }}^{\dagger}\right|\left|U_{\text {get }}\right|\right)$ is $F=0.98$. The processor preserves the second-order coherence of the photons [46].

In this experiment, we use the large size, coherence-preserving properties and high degree of experimental control over the interferometer to simultaneously implement a quantum simulation of thermalization and a verification experiment, in two separate sections of the interferometer. These two areas are indicated in blue and yellow, respectively in Fig. 8.2; the area below the dotted line in Fig. 8.2 is not used. These two areas both form individual universal interferometers on the restricted space of four optical modes, allowing us to apply two arbitrary optical transformations $U_{1}$ and $U_{2}$ in sequence. We use $U_{1}$ to simulate time evolution of our input state. We select two families of Hamiltonians to simulate: a hopping Hamiltonian which consists of equal-strength nearest-neighbor interactions between all modes, which simulates the superfluid, non-interacting limit of the Bose-Hubbard model, and a set of 20 randomly chosen long-range Hamiltonians, which we generate by applying the matrix logarithm to a set of Haar-random unitary matrices generated by standard techniques [279].

The second section of the interferometer, indicated in Fig. 8.2 in yellow, is used for certification. When we wish to directly measure the quantum state generated by the first area, we set this area to the identity, leaving the state after $U_{1}$ untouched. However, we can also use this second interferometer to make measurements in an arbitrary basis on the quantum state generated by $U_{1}$, which allows us to certify the closeness of our produced quantum state to the ideal case. In particular, when we chose $U_{2}=U_{1}^{-1}$, we undo the dynamics of the first interferometer. In the context of this experiment, this is a rather powerful choice of measurement setting, since if we can undo the evolution of $U_{1}$ we have demonstrated that any entanglement generated in the first time evolution was due to interaction between the optical modes as opposed to the interaction between the modes and the environment. This intuition forms the basis of our certification protocol.

Our photon source is a pair of periodically poled potassium titanyl phosphate (ppKTP) crystals operated in a Type-II degenerate configuration, converting light from $775 \mathrm{~nm}$ to $1550 \mathrm{~nm}$ [245]. Each crystal produces a two-mode squeezed vacuum state at $1550 \mathrm{~nm}$, with a bandwidth of $\Delta \lambda \approx 20 \mathrm{~nm}$. By using a single external herald detector and conditioning on the detection of 3 photons after the chip, we postselect on the state vector $|\Psi\rangle=|1110\rangle$ [20].

By tuning the relative arrival times of our photons, we can continuously tune the degree of distinguishability between our photons. Off-chip measurements via the Hong-OuMandel [133] effect lower bound the wave function overlap of our photons to $\left|\left\langle\psi_{i} \mid \psi_{j}\right\rangle\right|^{2}=$ $88.5 \%$ between sources, $93.2 \%$ for photons from the same source. Photon detection is 
achieved with a bank of 13 superconducting single-photon detectors [246, 247], which are read out with standard correlation electronics. For each of our four modes of interest, we multiplex three detectors to achieve quasi-photon number resolution [248].

\subsection{Results}

Figure 8.3 shows the results of our quantum simulation of the hopping Hamiltonian and 20 random instances of longe-range Hamiltonians, in subfigures a) and b), respectively. The two subfigures each have a tabular structure, where the columns indicate the different simulated timesteps, with the simulation time indicated at the head of the column, and the rows indicate different measurement settings, i.e., either the experiment itself or the corresponding certification measurements. The data in these figures was acquired over 20 minutes for the photon number distribution, 320 minutes per certification measurement for the hopping Hamiltonian and 220 minutes for each certification measurement of the long-range Hamiltonian.

The first row of the two subfigures displays the single-mode photon-number statistics $P(k)$ as generated after the application of $U$ in the first section of the processor. The output statistics were measured for the first output mode. The experiment was carried out for both distinguishable (blue points) and indistingusihable (red points) photons. The grey bars in the background denote the expected thermal distribution, also known as the canonical distribution, for our finite-size universe ${ }^{2}$.

For both Hamiltonians, initially, the input state is still clearly present, as indicated by the high probability to observe exactly one photon in the observed output mode. However, entanglement builds up as time evolves, since the photons increasingly mingle. Consequently, for the indistinguishable photons, the initial input state evolves to a thermallike state at $\tau=1$. For the hopping Hamiltonian, at later times, $(\tau=2, \tau=5)$, the reversibility of the system, together with the finite size of our Hamiltonian gives rise to a recurrence, i.e. the state evolves back towards the initial input state [253, 254]. For the long-range Hamiltonian, in contrast, the long-range interactions push away recurrences to later timesteps. For both Hamiltonians, the distinguishable photons (which do not generate entanglement) do not approach the canonical thermal state, demonstrating the intrinsic link between entanglement and thermalization.

The second row of the two subfigures shows full the output-state distribution $P(\mu)$ after only the application of $U$, measured with indistinguishable photons. The bars in the background correspond to the expected distributions. For the long-range Hamiltonian, representative data from a single of our 20 independent Hamiltonians is plotted. From this data, it can be clearly seen that at the point of thermalization, the photons are spread over many possible output configurations, whereas a recurrence manifests as a transition back to fewer possible output configurations.

The third and fourth rows show the output-state distributions after the first and second certification measurement, respectively. In these rows, the output configurations which

2 See the Appendices 

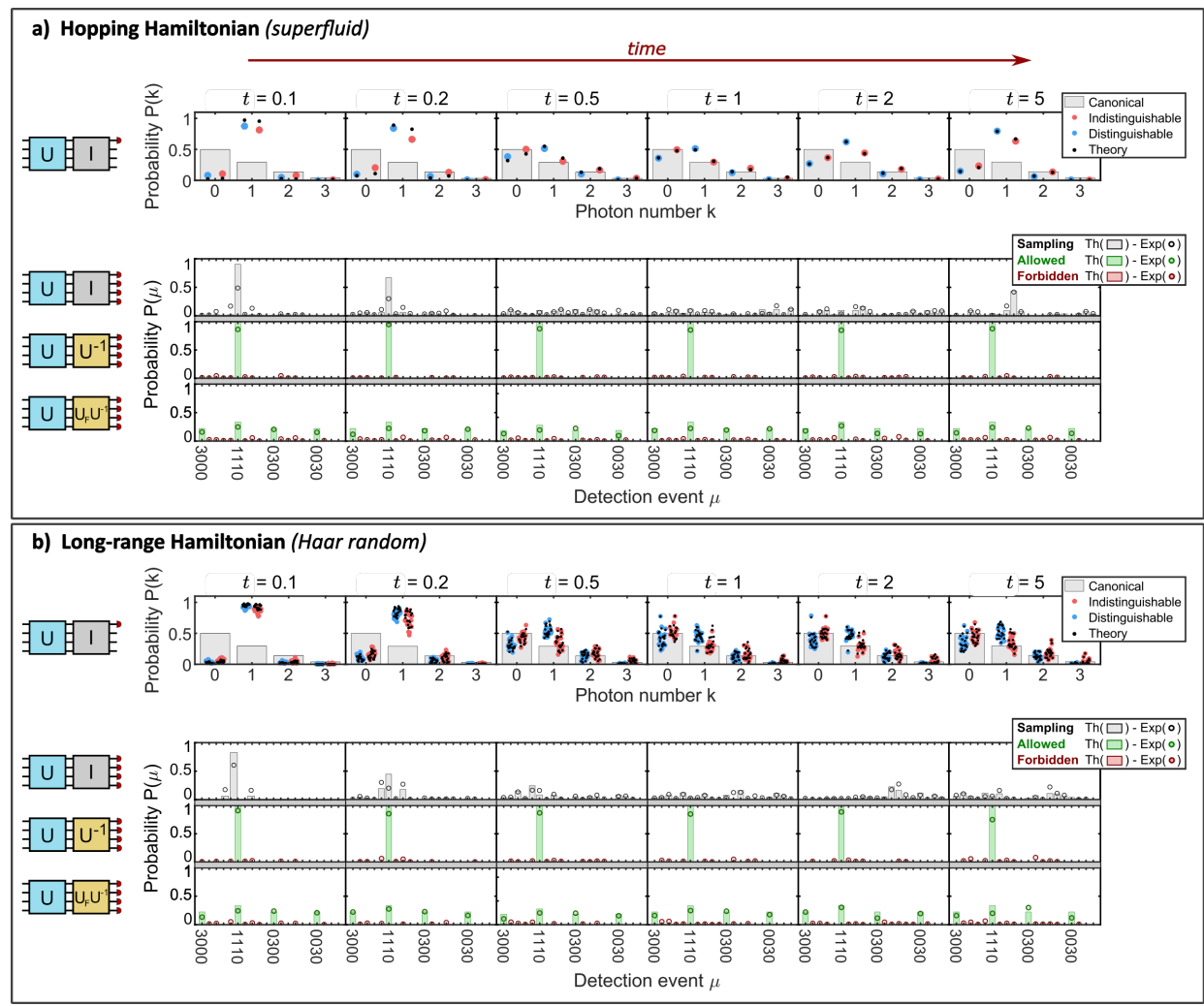

Figure 8.3 a) Hopping Hamiltonian: In the top panels, the time evolution of photon-number probability distribution in spatial output mode 1 is plotted. The black poins (squares) show the theoretical prediction for indistinguishable (distinguishable) particles, while colored points correspond to experimental data. The second to fourth rows show the observed output distributions. These rows correspond to the output distributions of the hopping Hamiltonian, the first certification measurement $U^{-1}$ and the second certification measurement $F U^{-1}$. The green-colored data corresponds with outcomes that benefit the certification protocol, whereas the red data is forbidden, i.e. ideally should not occur. b) Long-range Haar random model: In the top panels the time evolution of photon-number probability distribution in spatial output mode 1 for 20 different random Hamiltonians are plotted. The black points (squares) show the theoretical prediction for indistinguishable (distinguishable) particles, while colored points correspond to experimental data. The second to fourth rows show the observed output distributions for the first longe-range Hamiltonian. These rows correspond to the output distributions of the hopping Hamiltonian, the first certification measurement $U^{-1}$ and the second certification measurement $F U^{-1}$. The green-colored data corresponds with outcomes that benefit the certification protocol, whereas the red data is forbidden, i.e. ideally should not occur. 
contribute positively to the fidelity witness are indicated in green, and those which contribute negatively are indicated in red. The first certification measurement undoes the entanglement generated by $U$ and ideally only results in $\left|\psi_{\text {out }}\right\rangle=|1110\rangle$. The second certification measurements also applies a three-mode Fourier $U_{F}$ to the generated states. Ideally, this results in only four allowed output configurations. These certification measurements show good agreement with the ideal allowed states, demonstrating the high degree of control over the experiment. For the second certification measurement, most of the deviations from the expected distribution can be attributed to the known photon indistinguishabilities. From the data presented in the third and fourth row, we extract the values of $p_{1}$ and $p_{2}$, respectively, which are used in the fidelity witness (Eq 8.5).

\subsubsection{Certification}

Figures 8.4a) and $8.4 \mathrm{~b}$ ) show the lower bounds of certification fidelities with a probability $\epsilon=0.95$ of both the hopping Hamiltonian and the first random long-range Hamiltonian, respectively. The dashed and dotted lines are the thresholds of the uneven and even entanglement witness, respectively. Any experimental data point above either of these lines constitutes to a certification of entanglement for that time step. There are two different witness fidelities. The even bipartition witness is the fidelity corresponding to the situation where the photons in two output modes are entangled with photons in the remaining two output modes. The uneven bipartition entanglement witness certifies entanglement of photons in one output mode with those in the remaining three output modes.

Figure 8.4a) shows that entanglement is certified for $\tau=1$ in the hopping Hamiltonian system. The observed fidelity $F=0.359 \pm 0.005$ is above the threshold of the second entanglement witness, with 1 sigma. Similarly, Fig. 8.4b) shows an unambiguous certification for the first long-range Hamiltonian at $\tau=2$. The fidelity $F=0.363 \pm 0.014$ is well above the certification threshold.

The certification fidelities are limited by imperfect control over the processor. This follows from the certification fidelity at $\tau=0.2$ for the long-range Hamiltonian. This fidelity $F=0.463 \pm 0.012$ is significantly higher than others. Closer inspection shows a near optimal value for $p_{2}$, which is now only limited by the partial distinguishability of the generated photons. This implies that the certification at other time steps is limited by imperfect chip control, i.e. a limited fidelity at which any measurement can be implemented. A second factor limiting the certification is detector blinding, which affects the obtained values of $p_{1} \cdot{ }^{3}$

\subsection{Conclusion}

In conclusion, we have experimentally shown that a pure quantum state in a closed environment can locally behave like a thermal state because of entanglement with the

3 See the Appendices for more details on detector blinding and the convergence of the certification statistics. 
a) Hopping Hamiltonian (superfluid)

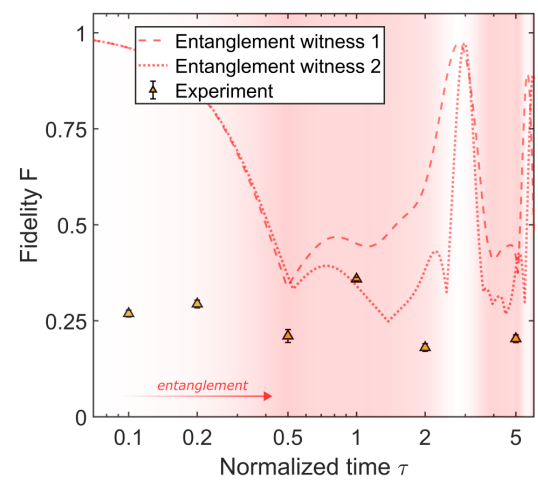

b) Long-Range Hamiltonian (Haar random)

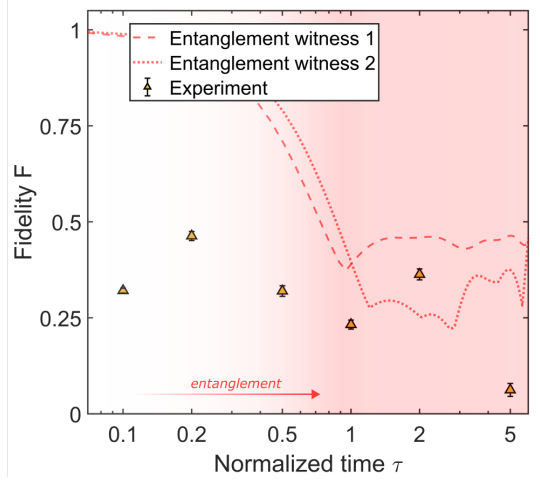

Figure 8.4 a) Certification of entanglement in hopping Hamiltonian (superfluid): The lower bound certification fidelity estimations for the hopping Hamiltonian are plotted against the simulated time for both an even and uneven bi-partition of the system. b) certification on entanglement in long-range Hamiltonian (Haar-random): The lower bound certification fidelity estimations are plotted against the simulated time for both an even and uneven bi-partition of the system. In both plots, the error bars correspond with 1 sigma deviation of uncertainty and the background color saturation is proportional to the total entanglement generated at that time step.

other modes. To this end, we simulated both the non-interacting limit of a Bose-Hubbard hopping Hamiltonian and 20 random long-range Hamiltonians on a programmable 12mode photonic processor. Previous experiments in this direction were irreversible since creating a sufficiently isolated quantum system is notoriously difficult. However, our experiments is -just like quantum mechanics itself- fully reversible. This reversibility allowed us to certify that the thermalization is due to entanglement between the quantum particles rather than with the environment. 


\section{ApPENDix 8}

\section{A Derivation of fidelity witness}

A fidelity witness provides guarantee that the fidelity of some target state with an experimental output is at least a certain threshold value [272]. Here we present a derivation of such a witness, including finite-size statistics, that is efficient in terms of experimental effort and classical computation. A natural question that arises is, what is a meaningful threshold. In this experiment, where the key feature of interest is the role of entanglement in producing local entropy production, we will use previously established relationships between fidelity and entanglement (see e.g. [277] to establish useful benchmarks). The idea is that the fidelity between a separable state and an entangled target state $\left|\Psi_{t}\right\rangle$ cannot exceed a certain threshold, which is set by the largest Schmidt coefficient. If the fidelity exceeds that threshold, i.e. if $F>\lambda_{\max }^{2}$, entanglement must be present. Because the size of the largest Schmidt coefficient decreases with the amount of entanglement, for more entangled states lower fidelities are sufficient to witness the presence of entanglement.

Ideal case: Fully-mode-resolving detectors. The fidelity between a quantum state $\rho$ and a target state $\sigma=|\Psi\rangle\langle\Psi|$ is defined as

$$
F(\rho, \sigma)=\operatorname{tr}(\rho \sigma)=\langle\Psi|\rho| \Psi\rangle
$$

In our case, the target state is an $n$ photons state $|n\rangle$ evolved by a unitary $U$, i.e. $\sigma=$ $|\Psi\rangle\langle\Psi|=U| n\rangle\langle n| U^{\dagger}$. Thus, the fidelity can then be written as

$$
F=F\left(\rho, U|n\rangle\langle n| U^{\dagger}\right)=F\left(U^{\dagger} \rho U,|n\rangle\langle n|\right)
$$

and is lower bounded in terms of photon number operators by [272]

$$
F^{(n)}=\left\langle(n+1-\hat{n}) \prod_{j=1}^{n} \hat{n}_{j}\right\rangle_{U^{\dagger} \rho U} .
$$

When one pre-selects the photon source for a same global photon number in each run (which we do in our experimental setup, to $n=3$ ), then $\langle\hat{n}\rangle=n$ and the bound simplifies to

$$
F^{(n)}=\left\langle\prod_{j=1}^{3} \hat{n}_{j}\right\rangle_{U^{+} \rho U^{\prime}}
$$

where $\hat{n}_{j}=\sum_{n_{j}=0}^{\infty}\left|n_{j}\right\rangle\left\langle n_{j}\right|$ are projective photon number operators, whose eigenvalues are the number of photons in mode $j$. Note that the only calculation necessary is to compute the hermitian conjugate of the given matrix, $U$.

Real case: Partially-mode-resolving detectors. In our experimental setup we do not have fully-mode-resolving detectors, meaning that we have no physical equivalent of $\hat{n}_{j}=\sum_{n_{j}=0}^{\infty}\left|n_{j}\right\rangle\left\langle n_{j}\right|$. In particular, our detectors can only resolve spatial modes and no temporal ones. This leaves an uncertainty regarding the exact mode of the photon after 
the measurement. Instead of projecting onto a unique mode (a single pure quantum state $M_{k}=|k\rangle\langle k|$ ), our detectors project onto a set of states, which are spread out over the temporal degrees of freedom and, without further work at least, cannot be distinguished. This uncertainty severely limits the fidelity that can be established. For three temporal modes, the measurements at each of the four spatial modes correspond to the following set of operators,

$$
\begin{aligned}
M_{0} & =|000\rangle\langle 000| \\
M_{1} & =|100\rangle\langle 100|+| 010\rangle\langle 010|+| 001\rangle\langle 001| \\
M_{2} & =|200\rangle\langle 200|+| 020\rangle\langle 020|+| 002\rangle\langle 002| \\
& +|101\rangle\langle 101|+| 110\rangle\langle 110|+| 011\rangle\langle 011| \\
M_{3} & =|300\rangle\langle 300|+| 030\rangle\langle 030|+\ldots,
\end{aligned}
$$

where $M_{0}$ measures no photons and $M_{1}, M_{2}$ and $M_{3}$ measure one, two and three photons, respectively, in a given spatial mode. Since we set up our experiment such that the ideal initial and the target state consists of one photon per spatial mode, our measurement operator of interest will be $M_{1}$ and our certification scheme will be based on the measurement of $M_{1}^{A} \otimes M_{1}^{B} \otimes M_{1}^{C} \otimes M_{0}^{D}$, where $A, B, C$ and $D$ label the four spatial modes. We will want to verify whether the initial state is being recovered after implementing the unitary and its inverse, i.e. that the three photons are in the same temporal mode and each in a different one of the first three spatial modes.

The problem with using these measurement and naievely applying the bound in Eq. (8.10) is that, considering Eq. (8.11), it is clear these measurements can produce the ideal click pattern even if the photons were completely distinguishable and no quantum interference or entanglement was present.

At this point we introduce some alternative notation that will come in handy later: The numbers in the ket-vector label the occupied temporal mode (there are three temporal modes, so the numbers go from 1 to 3 ) and the subscript 1 indicates that each spatial mode is occupied by one photon only (which is the case for $M_{1}$ ). For example, all photons being in the first temporal mode reads $|1,1,1\rangle_{1}=|100,100,100,000\rangle$. The first photon in the first, second photon in the second, and third photon in the third temporal modes reads $|1,2,3\rangle_{1}=|100,010,001,000\rangle$.

Now that we have pointed out the ambiguity of just counting photons in spatial modes, and equipped with useful notation, we next turn to the question of how to overcome the uncertainty regarding the temporal degree of freedom. An answer lies in the observation that certain interference patterns can be clearly associated with non-synchronous, i.e. distinguishable, photon states (similar to a HOM dip [133]) - we call those interference patterns forbidden patterns, $M_{f}$. We make use of this effect in practice by implementing a Fourier transform $U_{F}$ after the unitary $U$ and its inverse and use the overlap between the distinguishable subspace of states and the image of the forbidden patterns $M_{f}$ under the Fourier transform $F$ to sharpen the lower bound on the fidelity. It is worth mentioning that this overlap is not 1:1 and some ambiguity will remain. It does, however, reduce the ambiguity significantly and thereby increases the estimated fidelity in a useful way. 
We now give a full derivation of the fidelity bound. The bound, as in Eq. 8.5 has two components. 1) A lower bound $p_{1}$ on seeing one photon per spatial mode and 2) an upper bound $p_{2}$ on the overlap of $\rho$ with the distinguishable subspace. Together these two pieces of information give insights into i) whether the implemented unitaries are in fact unitary and ii) what entanglement pattern is present between the unitary and its inverse. The two components correspond to two different measurement settings:

1. Implement the unitary and its inverse and count photons

2. Implement the unitary and its inverse, implement a Fourier transform (an interference experiment) and then count photons

First measurement setup. For simplicity and without loss of generality we fix $M_{1}^{A}$ to the first temporal mode, i.e. $M_{1}^{A}=|100\rangle\langle 100|$. The first measurement setup can be expressed as the operator product $|100\rangle\langle 100| \otimes M_{1}^{B} \otimes M_{1}^{C} \otimes M_{0}^{D}$. The overlap of the state $U^{\dagger} \rho U$ with $|100\rangle\langle 100| \otimes M_{1}^{B} \otimes M_{1}^{C} \otimes M_{0}^{D}$, i.e. the probability of seeing one photon in each of the first three spatial mode (regardless of the temporal modes), can be estimated experimentally with accuracy $\epsilon_{1}$. This estimation takes the form of a lower bound $p_{1}$ - the result of the first round of measurements.

$$
\operatorname{tr}\left[U^{\dagger} \rho U\left(|100\rangle\langle 100| \otimes M_{1}^{B} \otimes M_{1}^{C} \otimes M_{0}^{D}\right] \geq p_{1}\right.
$$

which expanded yields

$$
\begin{aligned}
& \operatorname{tr}\left[U^{\dagger} \rho U(\right. \\
& |1,1,1\rangle_{1}\left\langle 1,1,\left.1\right|_{1}+\mid 1,1,2\right\rangle_{1}\left\langle 1,1,\left.2\right|_{1}+\mid 1,1,3\right\rangle_{1}\left\langle 1,1,\left.3\right|_{1}+\right. \\
& |1,2,1\rangle_{1}\left\langle 1,2,\left.1\right|_{1}+\mid 1,3,1\right\rangle_{1}\left\langle 1,3,\left.1\right|_{1}+\mid 1,2,2\right\rangle_{1}\left\langle 1,2,\left.2\right|_{1}+\right. \\
& \left.|1,3,3\rangle_{1}\left\langle 1,3,\left.3\right|_{1}+\mid 1,2,3\right\rangle_{1}\left\langle 1,2,\left.3\right|_{1}+\mid 1,3,2\right\rangle_{1}\left\langle 1,3,\left.2\right|_{1}\right)\right] \\
& \geq p_{1} .
\end{aligned}
$$

The first term (black) reflects the fidelity given by $F=\operatorname{tr}\left[U^{\dagger} \rho U\left(|1,1,1\rangle_{1}\left\langle 1,1,\left.1\right|_{1}\right)\right]\right.$. The other terms correspond to the overlap of $U^{\dagger} \rho U$ with those states where one (blue) or two (red) photons are distinguishable. We summarize those states as $\hat{P}_{1}$ and $\hat{P}_{2}$, respectively, and call $\hat{P}=\hat{P}_{1}+\hat{P}_{2}$ the distinguishable subspace. With that shorthand notation Eq. 8.13 simplifies to

$$
F \geq p_{1}-\operatorname{tr}\left(U^{\dagger} \rho U \hat{P}\right) .
$$

We can calculate $p_{1}$ by counting the relative number of instances of $M_{1}$ in our first measurement setup.

Second measurement setup. Next, in order to improve the fidelity bound in Eq. 8.14 we need to upper bound its second term $\operatorname{tr}\left(U^{\dagger} \rho U \hat{P}\right)$, which enters the fidelity bound with a minus sign. This term contains the overlap of $\rho$ with the distinguishable subspace $\hat{P}$. We upper bound it by implementing a Fourier interference experiment $U_{F}$, for which some counting patterns (the forbidden patterns) corresponding out states $M_{f}$ are impossible for 
perfectly indistinguishable photons [274-276]. This kind of discrimination is something we could not do in the first setup. The bound we can establish experimentally with our second measurement setup is

$$
\operatorname{tr}\left(U_{F} U^{\dagger} \rho U U_{F}^{-1} M_{f}\right) \leq p_{2}
$$

where $p_{2}$ is the the overlap of $U^{\dagger} \rho U$ with the image of the forbidden states based on the photon counting measurements (in particular the fraction of forbidden patterns).

In this case, the forbidden patterns are all those which are not either of $|1110\rangle,|3000\rangle$, $|0300\rangle,|0030\rangle$ [274-276]. We can use the cyclicity of the trace to obtain

$$
\operatorname{tr}\left(U^{\dagger} \rho U U_{F}^{-1} M_{f} U_{F}\right) \leq p_{2}
$$

and interpret the bound as one of the overlap of $\rho$ with the image of the forbidden states $M_{f}$ under the chosen Fourier transform. As mentioned before, some states within that image clearly correspond to distinguishable states, that is, states in $\hat{P}$ and other do not. To be more clear, we can express Eq. 8.16 in terms of the distinguishable subspaces $P_{i} \in \hat{P}$ $(i \in\{1,2\})$ in the following way

$$
\operatorname{tr}\left(U^{\dagger} \rho U \sum_{i} \lambda_{i} P_{i}\right) \leq \operatorname{tr}\left(U^{\dagger} \rho U U_{F}^{-1} M_{f} U_{F}\right) \leq p_{2}
$$

where $\lambda_{i}=\operatorname{tr}\left(P_{i} U_{F}^{-1} M_{f} U_{F}\right)$ is the probability that a state in the distinguishable subspace $P_{i} \in \hat{P}$ results in a forbidden pattern. For symmetry reasons (the Fourier transform is highly symmetric) all states both within $P_{1}$ and $P_{2}$ have equal probabilities $\lambda_{1}=4 / 9$ and $\lambda_{2}=2 / 3$ respectively. To calculate these probabilities all that really matters is the number of mutually distinguishable photons. For example, to calculate the probability of the forbidden state $|2010\rangle$ with one photon distinguishable from two other identical photons, one first computes the probability of the indistinguishable photons resulting in a state that could be transformed into a forbidden pattern the by the third photon. For this example that would be the outputs $|1010\rangle$ or $|2000\rangle$. Then one computes the probability that the final, distinguishable photon, would fall in the correct mode to produce the forbidden output, namely $|1000\rangle$ and $|0010\rangle$ respectively. The transition probabilities for distinguishable and indistinguishable photons through a Fourier matrix can be computed via [275]. Note that although the complexity of this computation is not completely clear, this computation should not be counted in the cost of the certification scheme since it is a one-off calculation that depends only upon the properties of a Fourier transform, not the unitary evolution to be certified and can hence be done offline.

Next we make use of the following property of the trace of sums of projections with scalar pre-factors $\lambda_{i} \geq 1$,

$$
\operatorname{tr}\left(U^{\dagger} \rho U\left(P_{1}+P_{2}+\ldots\right)\right) \leq \operatorname{tr}\left(U^{\dagger} \rho U\left(\lambda_{1} P_{1}+\lambda_{2} P_{2}+\ldots\right)\right)
$$


With this we can express Eq. 8.16 as

$$
\begin{aligned}
\operatorname{tr}\left(U^{\dagger} \rho U\left(\frac{4}{9} P_{1}+\frac{2}{3} P_{2}\right)\right) & \leq p_{2}, \\
\operatorname{Tr}\left(U^{\dagger} \rho U\left(P_{1}+\frac{3}{2} P_{2}\right)\right) & \leq \frac{9}{4} p_{2},
\end{aligned}
$$

where we have divided by $\frac{4}{9}$ and not by $\frac{2}{3}$, as the latter would have led to a pre-factor $\lambda_{i} \leq 1$

We can use Eq. 8.19 as an upper bound on $\operatorname{tr}\left(U^{\dagger} \rho U \hat{P}\right)=\operatorname{tr}\left(U^{\dagger} \rho U\left(P_{1}+P_{2}\right)\right)$, which is what we originally set out to do. We find that

$$
\operatorname{tr}\left(U^{\dagger} \rho U \hat{P}\right) \leq \operatorname{tr}\left(U^{\dagger} \rho U\left(P_{1}+\frac{3}{2} P_{2}\right)\right) \leq \frac{9}{4} p_{2}
$$

and thus arrive at the following final expression for the fidelity bound in Eq. 8.14:

$$
F \geq p_{1}-\operatorname{tr}\left(U^{\dagger} \rho U \hat{P}\right) \geq p_{1}-\frac{9}{4} p_{2}
$$

Finally, we turn to the question of finite-size statistics. This scheme is manifestly efficient in the number of measurement settings (two) but we must also consider the total sample size for each probability estimate. Many tools have been developed for this situation, and here we will make use of Chebyshev's inequality which states that given $k$ samples and an observed fraction $p_{1}$ we can say that the 'true' probability of that outcome, $\bar{p}_{1}$, must satisfy

$$
\operatorname{Pr}\left[\left|\bar{p}_{1}-p_{1}\right| \leq \delta\right] \geq \varepsilon, \quad \delta(\varepsilon)=\sqrt{\frac{2 V}{k \log (1 / \varepsilon)}},
$$

where $V$ is the variance of the distribution and $c$ is the number of measurements. This can be put together to obtain Eq. (8.5) which holds with probability, $\epsilon=\epsilon_{1} \epsilon_{2}$, and $\delta(\epsilon)=\delta\left(\epsilon_{1}\right)+\delta\left(\epsilon_{2}\right)$ arising from applying Eq. (8.22) to the experimental observations of $p_{1}$ and $p_{2}$.

\section{B Local Photon Statistics in Finite-size systems}

Local equilibrium photon statistics is determined by mutual photon (in)distinguishability. Temporal degrees of freedom are exploited for controlled tuning between distinguishable (asynchronized arrival time) and indistinguishable (synchronized arrival time) photons. Since the photonic quantum processor is limited to spatial transformations, temporal tuning allows for conveniently switching on and off any possible effects of spatial entanglement. Therefore, our photonic quantum processor is a convenient platform to study the effect of entanglement in multimode systems.

Behind one particular mode, the local maximum entropy state appears as if the global state $\rho$ is actually maximally mixed in all $m$ bosonic degrees of freedom. Switching to 
the ordered mode assignment list representation $\left(m_{1}, \ldots, m_{n}\right)$ (equivalent to Fock state representation $\left|n_{1}, \ldots, n_{m}\right\rangle$ ), the reduced global state is simply found by counting the number of lists in which the first $k$ boson are placed in (without loss of generality) mode 1 , while all $n-k$ leftover bosons are not. By construction, the latter allows for $m-1$ possibilities, resulting in a local maximum entropy state given by

$$
\Omega=\sum_{k=0}^{n} \frac{\left(\begin{array}{c}
n-k+m-2 \\
n-k
\end{array}\right)}{\left(\begin{array}{c}
n+m-1 \\
n
\end{array}\right)}|k\rangle\langle k|
$$

where $\Omega$ describes the statistics for a local equilibrium characterized by mean boson number $\bar{n}=\frac{n}{m}$ (all probabilities are normalized by global state space dimensionality $\left(\begin{array}{c}n+m-1 \\ n\end{array}\right)$ ). In the experiments, a system size of $m=4$ spatial modes is realized to illustrate the effect of entanglement on local equilibrium photon statistics. Moreover, we will show that for sufficiently large $m$ and conserved mean boson number the local maximum entropy state follows Bose-Einstein statistics

$$
\Omega=\sum_{k=0}^{n} \frac{\bar{n}^{k}}{(\bar{n}+1)^{k+1}}\left\{1-\frac{1}{1+\bar{n}} \frac{k^{2}-k}{2 \bar{n}} \frac{1}{m}+\mathcal{O}\left(\frac{1}{m^{2}}\right)\right\}|k\rangle\langle k| .
$$

For indistinguishable photons, a maximally mixed global state predicts that all threephoton output states are equiprobable. Based on Eq. 8.23, the local photon number distribution for indistinguishable photons is given by

$$
P_{\text {indistinguishable }}(k)=\frac{\left(\begin{array}{c}
n-k+m-2 \\
n-k
\end{array}\right)}{\left(\begin{array}{c}
n+m-1 \\
n
\end{array}\right)} \text {. }
$$

For distinguishable photons, the local photon number statistics can be thought of as a Bernoulli trial repeated $n$ times with success rate $\frac{1}{m}$. The local equilibrium is reflected in the success rate $\frac{1}{m}$, as in equilibrium each photon is equiprobable to be found behind each spatial mode. This results in a local photon number distribution for distinguishable photons given by

$$
P_{\text {distinguishable }}(k)=\left(\begin{array}{l}
n \\
k
\end{array}\right)\left(\frac{1}{m}\right)^{k}\left(1-\frac{1}{m}\right)^{n-k}
$$

Although both (in)distinguishable local photon number distributions predict a mean photon number $\bar{n}=\frac{n}{m}$, the distributions are different. This difference is illustrated in Fig. 8.5 for a finite-size system of $n=3$ photons and $m=4$ modes.

To explore the local maximum entropy state in the asymptotic limit of infinite bosonic 


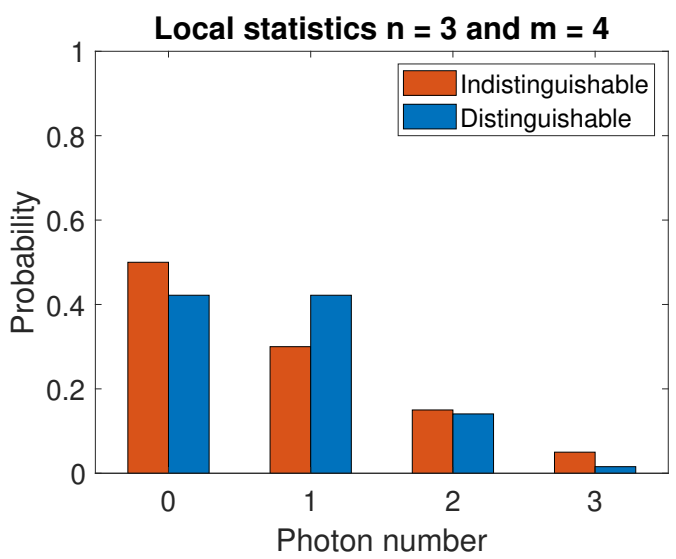

Figure 8.5 Expected equilibrium local photon number distributions for $n=3$ photons and $m=4$ modes. $P(k)=1$ is more likely for distinguishable photons as quantum interference suppresses anti-bunching.

degrees of freedom $m$, we rewrite Eq. 8.25 as

$$
\begin{aligned}
\frac{\left(\begin{array}{c}
n-k+m-2 \\
n-k
\end{array}\right)}{\left(\begin{array}{c}
n+m-1 \\
n
\end{array}\right)} & =\frac{(m+n-k-2) !}{(m-2) !(n-k) !} \frac{(m-1) ! n !}{(m+n-1) !} \\
& =(m-1) \frac{n !}{(n-k) !} \frac{(m+n-k-2) !}{(m+n-1) !} \\
& =\frac{m-1}{m+n-1} \frac{n !}{(n-k) !} \frac{(m+n-k-2) !}{(m+n-2) !} \\
& =\frac{1}{n+1} \frac{n !}{(n-k) !}\left(\frac{(m+n-2) !}{(m+n-2-k) !}\right)^{-1},
\end{aligned}
$$

where $\dot{n} \equiv \frac{n}{m-1}$ and additionally $\ddot{n} \equiv \frac{n}{m-2}$ are defined for convenience.

A common factor $(n-k)$ ! cancels out in the second fraction of Eq. 8.30 resulting in

$$
\begin{aligned}
\frac{n !}{(n-k) !}=\frac{n \cdot(n-1) \cdot \ldots \cdot(n-(k-1)) \cdot(n-k) !}{(n-k) !} \\
\quad=\prod_{j=0}^{k-1}(n-j) \\
=\prod_{j=0}^{k-1} n\left(1-\frac{j}{n}\right) \\
=n^{k} \prod_{j=0}^{k-1}\left(1-\frac{j}{n}\right) .
\end{aligned}
$$

Similarly,

$$
\frac{(m+n-2) !}{(m+n-2-k) !}=(m+n-2)^{k} \prod_{j=0}^{k-1}\left(1-\frac{j}{m+n-2}\right) .
$$


Substituting Eq. 8.34 and Eq. 8.35 in Eq. 8.30, we find

$$
\begin{aligned}
\frac{\left(\begin{array}{c}
n-k+m-2 \\
n-k
\end{array}\right)}{\left(\begin{array}{c}
n+m-1 \\
n
\end{array}\right)}= & \frac{1}{\dot{n}+1}\left(\frac{\frac{n}{m-2}}{1+\frac{n}{m-2}}\right)^{k}\left\{\prod_{j=0}^{k-1} \frac{1-\frac{j}{n}}{1-\frac{n}{m+n-2} \frac{j}{n}}\right\} \\
= & \frac{1}{\dot{n}+1}\left(\frac{\ddot{n}}{\ddot{n}+1}\right)^{k}\left\{\prod_{j=0}^{k-1} \frac{1-\frac{j}{n}}{1-\frac{n}{m+n-2} \frac{j}{n}}\right\} \\
& \approx \frac{\bar{n}^{k}}{(\bar{n}+1)^{k+1}}\left\{\prod_{j=0}^{k-1} \frac{1-\frac{j}{n}}{1-\frac{n}{m+n} \frac{j}{n}}\right\} \\
& \approx \frac{\bar{n}^{k}}{(\bar{n}+1)^{k+1}}\left\{\prod_{j=0}^{k-1} \frac{1-\frac{1}{\bar{n}} \frac{j}{m}}{1-\frac{1}{1+\bar{n}} \frac{j}{m}}\right\},
\end{aligned}
$$

for sufficiently large $m$. Anticipating the asymptotic limit, expanding the argument between curly brackets around $\frac{1}{m}=0$ gives

$$
\begin{aligned}
\prod_{j=0}^{k-1} \frac{1-\frac{1}{\bar{n}} \frac{j}{m}}{1-\frac{1}{1+\bar{n}} \frac{j}{m}}= & \prod_{j=0}^{k-1}\left(1+\left(\frac{1}{1+\bar{n}}-1\right) \frac{j}{m}+\mathcal{O}\left(\frac{1}{m^{2}}\right)\right) \\
= & \prod_{j=0}^{k-1}\left(1-\frac{1}{1+\bar{n}} \frac{j}{m}+\mathcal{O}\left(\frac{1}{m^{2}}\right)\right) \\
= & 1-\frac{1}{1+\bar{n}}\left(\sum_{j=0}^{k-1} j\right) \frac{1}{m}+\mathcal{O}\left(\frac{1}{m^{2}}\right) \\
= & 1-\frac{1}{1+\bar{n}} \frac{k(k-1)}{2} \frac{1}{m}+\mathcal{O}\left(\frac{1}{m^{2}}\right) \\
& =1-\frac{1}{1+\bar{n}} \frac{k^{2}-k}{2 \bar{n}} \frac{1}{m}+\mathcal{O}\left(\frac{1}{m^{2}}\right) .
\end{aligned}
$$

Substituting Eq. 8.44 in Eq. 8.39 eventually results in

$$
\Omega=\sum_{k=0}^{n} \frac{\bar{n}^{k}}{(\bar{n}+1)^{k+1}}\left\{1-\frac{1}{1+\bar{n}} \frac{k^{2}-k}{2 \bar{n}} \frac{1}{m}+\mathcal{O}\left(\frac{1}{m^{2}}\right)\right\}|k\rangle\langle k| .
$$

which, for fixed mean photon number $\bar{n}=\frac{n}{m}$, converges to Bose-Einstein statistics for sufficiently large bosonic degrees of freedom $m$.

By definition, the temperature $T$ of a thermal equilibrium between a local system and multimode environment is given by evaluating the energy derivative of the local system entropy $S$ at its expected energy level $\langle E\rangle$ :

$$
\frac{1}{T}=\left.\frac{d S}{d E}\right|_{E=\langle E\rangle}
$$

The non-interacting local Hamiltonian acting on the local state gives $H|k\rangle=\epsilon k|k\rangle$, such that $\langle E\rangle=\epsilon \bar{n}$. The local system entropy when occupied by $k$ photons can be found by noting that for the maximum local entropy state, all global states $\left|n_{1} n_{2} n_{3} n_{4}\right\rangle$ are equiprobable. Therefore, the number of global states $W$ corresponding to $k$ photons 
locally is given by taking the inverse of Eq. 8.45. Thus, the system's Boltzmann entropy is given by

$$
\begin{gathered}
\quad=k_{B} \ln (W) \\
=-k_{B} \ln \left(\frac{\bar{n}^{k}}{(\bar{n}+1)^{k+1}}\left\{1-\frac{1}{1+\bar{n}} \frac{k^{2}-k}{2 \bar{n}} \frac{1}{m}+\mathcal{O}\left(\frac{1}{m^{2}}\right)\right\}\right) \\
=-k_{B}\{k \ln (\bar{n})-(k+1) \ln (\bar{n}+1)+R(k)\},
\end{gathered}
$$

where $R(k)=\ln \left(1-\frac{1}{1+\bar{n}} \frac{k^{2}-k}{2 \bar{n}} \frac{1}{m}+\mathcal{O}\left(\frac{1}{m^{2}}\right)\right)$. Using this expression, the temperature is given by

$$
\begin{gathered}
\frac{1}{T}=\left.\frac{d S}{d E}\right|_{E=\langle E\rangle} \\
=\left.\frac{d S}{d k}\right|_{k=\bar{n}}\left(\left.\frac{d E}{d k}\right|_{k=\bar{n}}\right)^{-1} \\
=\frac{k_{B}}{\epsilon}\left\{\ln \left(\frac{\bar{n}+1}{\bar{n}}\right)-\left.R^{\prime}(k)\right|_{k=\bar{n}}\right\} .
\end{gathered}
$$

Expanding $R^{\prime}(k)$ gives

$$
R^{\prime}(k)=-\frac{1}{1+\bar{n}} \frac{2 k-1}{2 \bar{n}} \frac{1}{m}+\mathcal{O}\left(\frac{1}{m^{2}}\right),
$$

which results after substitution in

$$
\frac{1}{T}=\frac{k_{B}}{\epsilon}\left\{\ln \left(\frac{\bar{n}+1}{\bar{n}}\right)+\frac{2 \bar{n}-1}{2 \bar{n}(1+\bar{n})} \frac{1}{m}+\mathcal{O}\left(\frac{1}{m^{2}}\right)\right\} .
$$

For sufficiently large $m$, it is easily shown that $\Omega \propto e^{-\frac{H}{k_{B} T}}$.

\section{C Experimental methods}

Simulating time evolutions. Arbitrary time evolutions of a bosonic multimode state $\left|n_{1} n_{2} n_{3} n_{4}\right\rangle$ subject to non-interacting quadratic Hamiltonians are mimicked by directly programming the corresponding unitary transformation $U$ of the bosonic ladder operators, $b_{\text {out }}=U b_{\text {in }}$. Here, the unitary transformation mimicks time evolution

$$
U=e^{-i H t},
$$

where the Hamiltonian $H$ can be any arbitrary non-interacting quadratic Hamiltonian

$$
H=\sum_{i, j} c_{i j} b_{i}^{\dagger} b_{j}
$$

with an $m$-by- $m$ Hermitian parameter matrix $C$ with entries $c_{i j}$. Physically, entries $c_{i j}$ represent (inter) modal on-site and hopping energies. In principle, there is no experimental limitation on the programmable range of values for $c_{i j}$ for time evolution simulations 
except for Hermitian requirement $c_{i j}^{\dagger}=c_{j i}$. In this work, the time evolution of a simple non-interacting Bose-Hubbard system is simulated by choosing $c_{i j}=1$ for $i=j$ and nearest neighbor modes (periodic boundary conditions applies) while $c_{i j}=0$ otherwise. A classical computer is used to compute the target unitary and corresponding settings for our photonic quantum processor for experimental implementation.

Exploiting the experimental freedom of choosing arbitrary (inter) modal energy strengths $c_{i j}$, we choose multimodal Hamiltonians by drawing random unitary matrices from the Haar-measure and connecting them implicitly by defining $U=e^{-i H}$. Haar-random unitaries $U_{\text {Haar }}$ ) are generated according to [279], while corresponding time evolution unitaries are clasically computed by taking matrix exponents as $U=\left(U_{\text {Haar }}\right)^{t}$. This particular choice of choosing Hamiltonians is motivated by the notion that input state information is expected to be wiped out for Haar-random unitaries, resulting in an expected uniform distribution over the global output states [89]. Since the input state information is expected to be wiped out, it seems reasonable to expect that recurrence timescales are well beyond local equilibration timescales. This is promising in experimentally simulating relaxation towards local equilibrium. Since target time evolution unitaries are classically computed, our platform allows for studying the effects of time-reversal. Time-reversal is easily achieved by substituting $t \rightarrow-t$ in all classical preparatory computations of target unitaries.

Switching between quantum and classical photon statistics is realized by engineering the spatiotemporal input mode. An (in)distinguishable $|1110\rangle$ state is prepared by heralding two combined free-space spontaneous parametric down conversion (SPDC) sources and introducing time delays for different spatial modes. Indistinguishability is prepared by matching all temporal modes. The prepared input state is subject to a user-programmed unitary operation coherently transforming spatial modes. Finally, spatial mode photon number resolving detectors count the occurrence rate of all microstates. This measured microstate frequency is used to estimate the global microstate probability distribution from which a local photon number probability distribution is inferred. Although the detectors cannot resolve temporal modes, temporal distinguishability still prevents spatial interference and thus precludes the effects of quantum interference within the observed statistics. Each simulation step is measured for 300s (pure measurement time excluding initialization overhead), such that estimated statistics is based on $>30,000$ samples resulting in a negligible small relative error.

Initial state preparation. (In)distinguishable $|1110\rangle$ states are generated by a multiphoton source formed by two free-space SPDC sources. Two non-linear $2 \mathrm{~mm}$ length ppKTP crystals (Raicol Crystals) are pumped by a Ti:Sa mode-locked laser (Tsunami, Spectra Physics) at $775 \mathrm{~nm}$ with a spectral bandwidth of $5.4 \mathrm{~nm}$ FWHM. Pulses are generated with a repetition frequency of $80 \mathrm{MHz}$ and $150 \mathrm{fs}$ pulse duration. Each crystal is pumped by approximately $10 \mathrm{~mW}$ pump power, generating degenerate signal-idler pairs 
at $1550 \mathrm{~nm}$ with generation probability $<1 \%$ per pulse. Typical heralding efficiencies for individual crystals are around $40-45 \%$, while typical two-photon event rates are $\sim 0.20$ $\mathrm{MHz}$ coincidence counts at $40 \mathrm{~mW}$ pump power.

Pumping a single ideal SPDC source results in a two-mode squeezed vacuum state (TMSV) given by

$$
\left|\psi_{S P D C}\right\rangle=\sqrt{1-\lambda^{2}} \sum_{n=0}^{\infty} \lambda^{n}\left|n_{s} n_{i}\right\rangle .
$$

Signal-idler pairs are generated with orthogonal polarization because of type II SPDC. Therefore, half wave plates are used to match polarization degrees of freedom. However, energy and momentum conservation results in spectral signal-idler correlations because of the periodically-poled structure of the non-linear crystals. These correlations are suppressed by using a spectral bandpass filter of $25 \mathrm{~nm}$.

By combining two identical SPDC sources, generating Fock states $|2200\rangle,|1111\rangle$ or $|0022\rangle$ is equiprobable. Therefore, one of the modes is used for heralding while the other three modes are fed to the processor. Three motorized linear stages (SLC-2475, Smaract $\mathrm{GmbH}$ ) are used to control relative photon arrival times, used to switch between (in)distinguishable photons. In the absence of severe experimental losses, post-selecting on heralded three-photon events results in selecting events initiated by input state $|1110\rangle$ with high certainty. To characterize the quality of our prepared indistinguishable $|1110\rangle$ input state, we measured inter-modal HOM-effects (Fig. 4.4). We find an average visibility of $V>88 \%$.

Number resolved detection. Spatial pseudo-number resolution detection is realized by multiplexed superconducting nanowire single photon detectors (SNSPDs), since individual SNPSDs are threshold detectors. 13 SNSPDs (PhotonSpot) are biased close to their critical current ( 8 to $22 \mathrm{uA}$ range), operating close to quantum efficiencies of $90 \%$ for $1550 \mathrm{~nm}$ photons with typical $200 \mathrm{~Hz}$ dark counts. Fourfold coincidence rates within a 750 ps window are monitore by a timetagger device (Timetagger Ultra, Swabian). Therefore, less than one in a million measured fourfold coincidence events are expected to be triggered by a dark count. Each output mode is connected to an effective 1-to-3 fibre beam splitter terminated by SNSPDs, realizing quasi three photon number resolving detection (q3PNRD) behind each spatial mode and leaving one SNSPDs for herald detection. Polarization maintaining (PM) fibers are used in combination with polarization controllers to optimize and stabilize output counts in each channel.

Non-uniform relative output losses are characterized to reduce biased microstate sampling. Although in-coupling efficiencies of each spatial mode might not be uniform, this is not relevant because each sampled microstate event originates from the same prepared input state $|1110\rangle$. Therefore, each measured microstate event suffers from the same input losses. Furthermore, we assume that on-chip losses are reasonably uniform which is evidenced by the high matrix amplitude fidelities. At the output side, however, microstate 
events have different output losses. Non-uniform losses such as outcoupling efficiencies, splitting ratios and detection efficiencies cause unequal output losses for each measured microstate event apart from probabilistic multi-photon detection effects. Hypothetically, some microstates may appear to be more likely than others due to different output losses despite the applied unitary transformation dictates equiprobability.

Non-uniform detection channel losses are characterized by fully transmitting heralded single photons from input mode 1 to all four output modes consecutively. Probing each output mode separately leaves the possibility to check how much light actually leaks into the other 'forbidden' output modes. We can arbitrarily choose input mode 1, since on-chip losses are assumed to be uniform and each measured microstate event originates from the input $|1110\rangle$ in which the first mode is filled. In each of these four consecutive experiments, the heralded singles count rate of each detector in the q3PNRD behind the output mode of interest is measured. All measured heralded singles count rates $S_{i}$ originate from the same on-chip uniform heralded single photon rate $R_{1}$, therefore it is convenient to pool all non-uniform losses such as outcoupling efficiencies, detection efficiencies and splitting ratios for each detection channel $i$ in a lumped factor $p_{i}$ :

$$
S_{i}=p_{i} R_{1} \text {. }
$$

Since we cannot obtain $R_{1}$ from these measurements, we introduce relative weight factors for each detection channel. These weight factors are normalized with respect to the maximum measured heralded singles rate and defined by:

$$
w_{i}=\frac{S_{i}}{S_{\max }} .
$$

Effectively, we find $p_{i}=w_{i} \cdot p_{\max }$. These weight factors are important to reduce bias in microstate sampling. In our experiments, we achieved excellent weight factor stability. Typically, we observed less than $1 \%$ relative fluctuations over more than $15 \mathrm{~h}$ time span.

Since the SNSPDs are threshold detectors, 1-to-3 fibre beam splitters are used to map a microstate $\left|n_{1} n_{2} n_{3} n_{4}\right\rangle$ onto a click/no click basis $\left|c_{1} c_{2} \ldots c_{12}\right\rangle$, where $c_{i} \in\{0,1\}$ and $\sum_{i} c_{i}=\sum_{i} n_{i}$. In the main experiments, we measure heralded threefold coincidence events $C C_{x y z}$ for which detectors $x, y$ and $z$ and the herald detector clicked simultaneously within a 1000 ps time interval. We place a q3PNRD behind each mode $i$, which is formed by three detectors $x_{i}, y_{i}$ and $z_{i}$. Our main question is how to map click events $C C_{x y z}$ onto unbiased microstate events $C C_{\left|n_{1} n_{2} n_{3} n_{4}\right\rangle}$. Based on combinatorial arguments, we find that a microstate $\left|n_{1} n_{2} n_{3} n_{4}\right\rangle$ maps to a specific combination of detector clicks. These specific click-combinations are obtained by choosing for each mode $n_{i}$ detectors out of the corresponding q3PNRD detector set $\left\{x_{i}, y_{i}, z_{i}\right\}$ and form combinations. All allowed combinations are described by $\sum_{(x, y, z)} C C_{x y z}$. We estimate the microstate probability based on occurrence frequencies:

$$
P(\mathbf{n})=\frac{C C_{\left|n_{1} n_{2} n_{3} n_{4}\right\rangle}}{\sum_{\mathbf{n}} C C_{\left|n_{1} n_{2} n_{3} n_{4}\right\rangle}} .
$$


Since $P(\mathbf{n})$ is normalized, only relative weight factors are important for unbiased sampling corrections as $p_{\max }^{3}$ drops out. Therefore, we will omit $p_{\max }$.

For probabilistic multi-photon detection, the probability of measuring $j$ photons behind mode $i$ when $k$ photons are injected, is given by $P_{i}(j \mid k)$. We note that for $P_{i}(1 \mid 1)$ and $P_{i}(2 \mid 2)$ there are three permutations possible, while for $P_{i}(3 \mid 3)$ there is just one permutation. More explicitly, we find:

$$
\begin{gathered}
P_{i}(0 \mid 0)=1, \\
P_{i}(1 \mid 1)=w_{x_{i}}+w_{y_{i}}+w_{z_{i}}, \\
P_{i}(2 \mid 2)=2 !\left(w_{x_{i}} w_{y_{i}}+w_{y_{i}} w_{z_{i}}+w_{z_{i}} w_{x_{i}}\right), \\
P_{i}(3 \mid 3)=3 ! w_{x_{i}} w_{y_{i}} w_{z_{i}},
\end{gathered}
$$

where $w_{x_{i}}+w_{y_{i}}+w_{z_{i}} \leq 1$ due to incorporated losses. Since all $P_{i}(j \mid k)$ are independent probability events, we eventually find for unbiased microstate event rates:

$$
C C_{\left|n_{1} n_{2} n_{3} n_{4}\right\rangle}=\frac{\sum_{(x, y, z)} C C_{x y z}}{P_{1}\left(n_{1} \mid n_{1}\right) P_{2}\left(n_{2} \mid n_{2}\right) P_{3}\left(n_{3} \mid n_{3}\right) P_{4}\left(n_{4} \mid n_{4}\right)} .
$$

These results are used to correct raw measurement data.

Detection limitations. Although post-selection on heralded three-photon events allows for extracting events based on $|1110\rangle$ input states, by-product states are omnipresent because of the probabilistic nature of SPDC sources. Unwanted by-product states cause detector blinding, which in combination with imperfect matrix fidelities biases observed photon statistics. This effect is illustrated in Fig. 8.6 for the identity matrix transformation. Therefore, observed photon statistics is dependent on used pump power levels. Especially photon statistics for unitary matrix transformations close to the identity matrix transformation are affected as can be seen in Fig. 2 (main text), as detector blinding is more likely due to the reliance on a limited set of detectors.

\section{D Statistics of certification}

The thermal-like states are certified with a probabilistic lower fidelity bound:

$$
F \geq p_{1}-\delta_{1}-\frac{9}{4}\left(p_{2}+\delta_{2}\right),
$$

and the error bounds on $p_{1}$ and $p_{2}$ are given by:

$$
\delta_{x}=\sqrt{\frac{2 p_{x}\left(1-p_{x}\right)}{N_{x} \ln (1 / \sqrt{\epsilon)}}}
$$




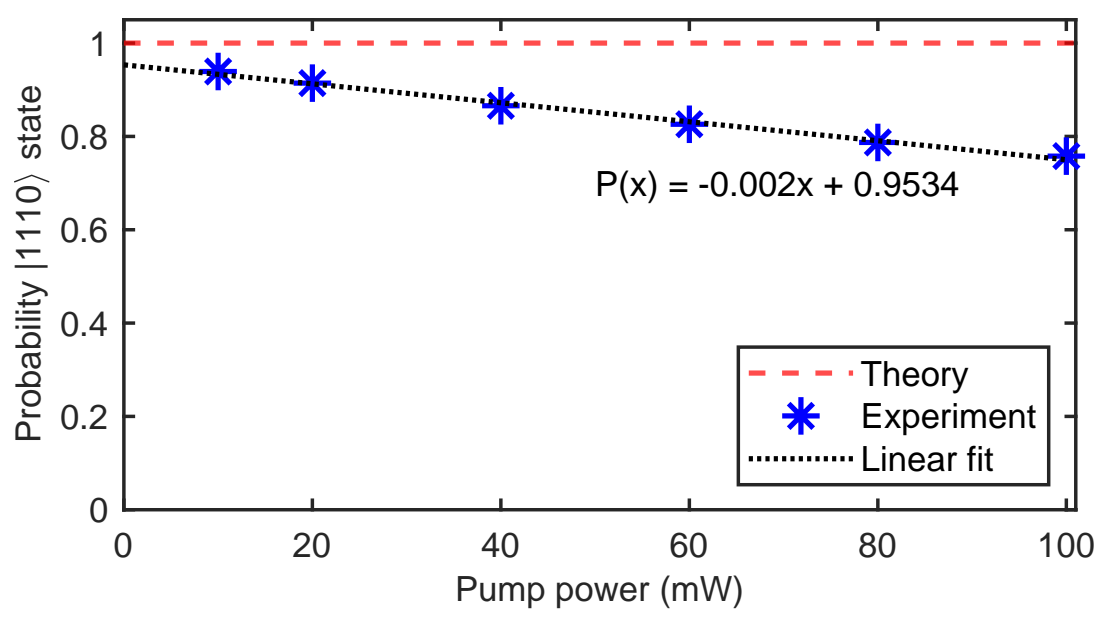

Figure 8.6 Detector blinding. Probability of measuring $|1110\rangle$ for the identity matrix transformation. For higher pump-power levels, the detrimental effect of by-products is increased. In case of perfect realized unitary fidelity, measurement probability is independent of pump power. Data is fitted by $P(|1110\rangle)=-0.0020 \cdot P_{\text {pump }}+0.9534$ with pump power $P_{\text {pump }}$ in $\mathrm{mW}$. The statistical error is within symbol size.

with $x=\{1,2\}, p_{1}\left(p_{2}\right)$ the observed success (fail) factor in the certification experiment with $N_{1}\left(N_{2}\right)$ heralded three-photon events, respectively.

The certification fidelity only becomes meaningful when the error bounds are sufficiently small. This requires prolonged measurement times to accumulate sufficient statistics. Moreover, the detector blinding (see Fig. 8.6) limits the average pump power for the fidelity certification measurements to only $5 \mathrm{~mW}$ per crystal. Such low pump power results in a fourfold coincidences rate of around $4 \mathrm{~Hz}$. To prevent a bias caused by longterm drift, all certified timesteps are measured 'interleaved', i.e. each certification measurement is repeated throughout multiple times for short run time.

Figures ?? and 8.7 show how the certification fidelities converge when the total measurement time is increased. Each datapoint is the result of a 20 minute measurement per certification measurement, i.e. 40 minutes per timestep. The $\mathrm{x}$-axis is linearized by $1 / \sqrt{\text { Time }}$, since $N_{x} \propto$ Time. The red horizontal dashed (dottes) lines are the even (uneven) bipartition fidelities required to certify the thermal-like state. Finally, the blue solid line is a linear fit through the datasets. The fit is extrapolated to 100 hours of measurement time. The number at the end (left) of the fit is the corresponding maximum fidelity expected based on this extrapolation.

Figure ?? shows the convergence of the certificiaton fidelity for the non-interacting Bose-Hubbard, or superfluid, Hamiltonian. Each panel corresponds with one of the six simulated timesteps. There are a total of 16 batches, which is sufficient to certify $\tau=1.0$ against the even bipartition. Furthermore, the extrapolations indicate that longer 
measurement times are not going to certify the remaining simulated timesteps.

Similarly, the first long-range Haar-random system's certification converges as shown in Fig. 8.7. Here, the simulated timestep of $\tau=2.0$ is clearly certified against the even bipartion and the linear fit through the data promises a possible certification against the uneven bipartition. The latter, however, requires a significant increase in measurement time. Unfortunately, the other timesteps will not be able to reach the required certification fidelities when the measurement time is increased. 
a)

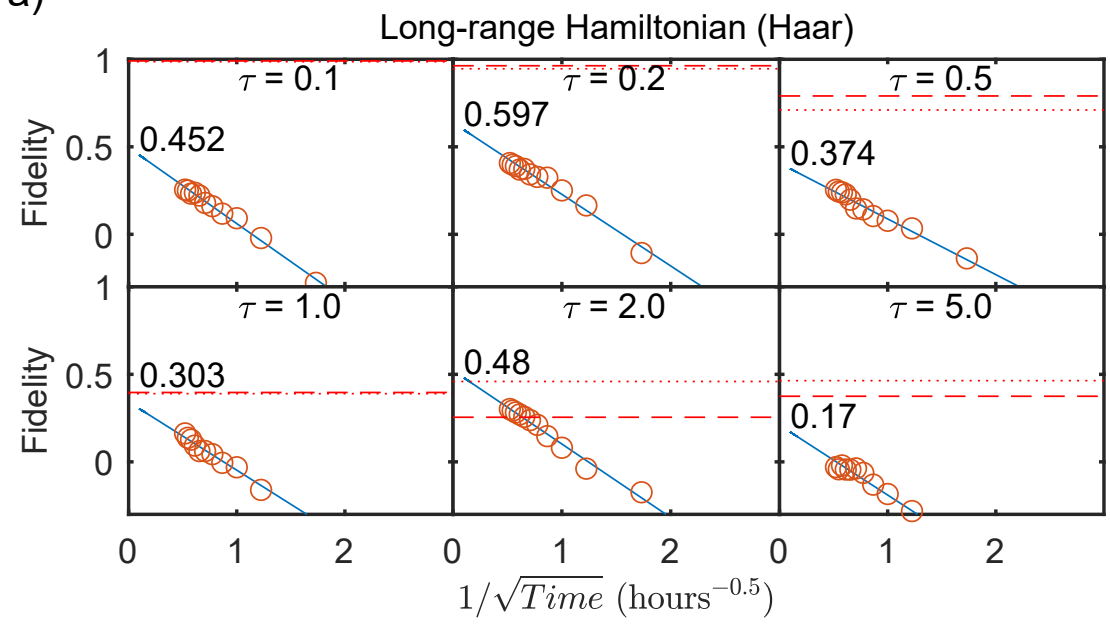

b)

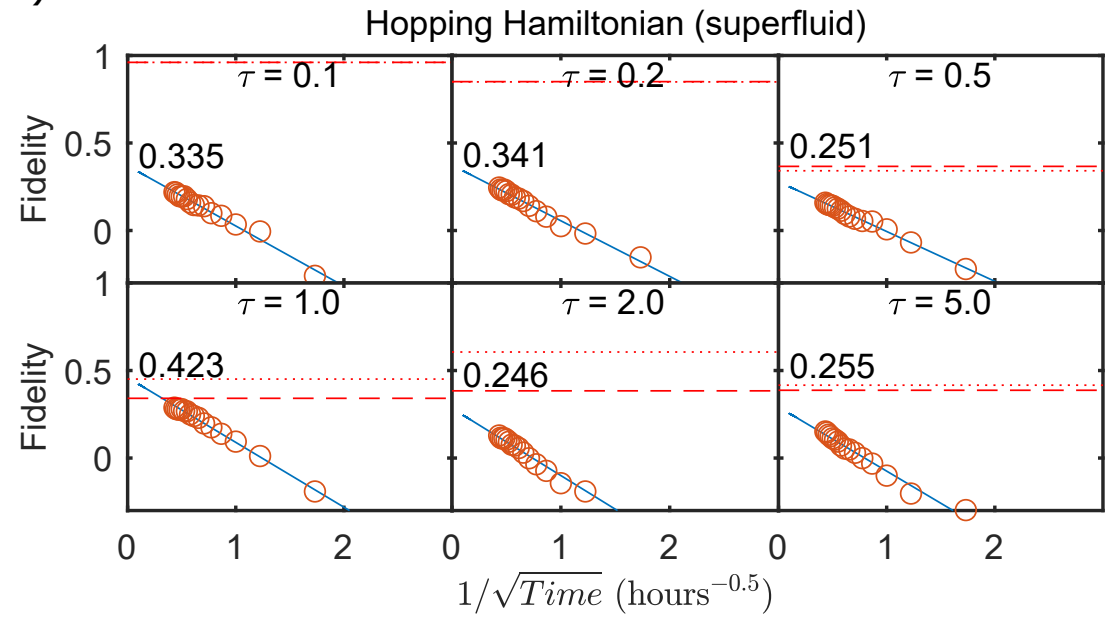

Figure 8.7 Convergence of certification statistics. a) Certification of the first long range Haar random Hamitonian range order system certification for all 6 timesteps. b) Certification of noninteracting Bose-Hubbard system certification for all 6 timesteps. In all plots, the dashed lines corresponds to the uneven bipartition and the number at the left is the extrapolated certification fidelity. 



\section{Summary and outlook}

\subsection{Summary}

When the work on this Ph.D. project started in 2018, the possibility of demonstrating a quantum advantage was being discussed, but this was mainly an academic discussion. The maximum number of photons in any experiment could still be counted on a single hand, the number of channels in the interferometers was barely any larger than the number of photons and the losses were simply too high. The situation has changed dramatically in recent years, which culminated in a claimed observation of the photonic quantum advantage. This triggered a surge in new developments towards larger low-loss interferometers, better multiphoton sources and detectors. This thesis is placed in this setting. It offers new insights into developments in photon sources, interferometers and demonstrates first applications thereof.

We firstly introduce an optical network that incorporates many modes with low losses. The network can be realized using a surface scatterer or phase plate. This network is not arbitrarily reconfigurable, but this is not strictly needed for boson sampling. This can be understood easily from the essence of boson sampling: it is not about doing something useful, it is about cutting as many corners as possible while maintaining computational complexity. Hence, we show the computational complexity of a surface scattering network by both theoretical and numerical results. Given the computational complexity, this type of network can be used to perform an improved photonic quantum advantage experiment that yields significantly lower losses while granting access to significantly more modes.

The next missing piece to do multiphoton experiments is a source that can generate many indistinguishable photons simultaneously. The traditional workhorse of quantum optics is spontaneous parametric downconversion, or SPDC in short, which uses nonlinear crystals. The suitability of SPDC for multiphoton experiments was an open question. We show the suitability of a few selected nonlinear crystals for SPDC. The optimal source turns out to be an aperiodically poled KTP crystal at $1582 \mathrm{~nm}$. This source can be multiplexed to produce 11500 photons that can still meaningfully interfere. These insights are incorporated in the design of our $1550 \mathrm{~nm}$ SPDC source. It has two periodically poled KTP crystals that each generates photon pairs at a rate of $0.2 \mathrm{MHz}$, with a heralding efficiency 0.45 and a purity of 0.89 . The photon quality of the source is good enough to interfere 11 photons quantum mechanically.

Multiphoton experiments require a large interferometric network to benefit from all these photons. The most promising way to build large, low-loss and tunable interferometers is integrated photonics. We demonstrate a state-of-the-art silicon nitride quantum processor. This processor has around $2.2 \mathrm{~dB}$ loss and can connect all 12 input modes to all 12 output modes in a fully reconfigurable manner. The fidelity at which any random interferometer can be implemented is around 0.99 for the amplitude matrix, as measured with a coherent laser light. The measured fidelity with our single photons is somewhat lower, just under 0.90. This photonic processor, together with the multiphoton setup, forms the heart of all experiments of this thesis.

The first experiment performed with the quantum processor is the demonstration of 
open channels in mesoscopic media. Open channels are the counter-intuitive prediction from the 1980s that wave propagation through disordered media is described by fully reflective and fully transmissive eigenmodes, closed and open channels respectively. Despite significant experimental effort, this prediction had not yet been convincingly observed in experiments. We show experimental proof of the existence of open channels. This was possible since the chip naturally allows access to all input and output modes, which turns out to be a prerequisite for observing these open channels.

With experimental proof that the photonic processor is well-suited for multiphoton experiments, a natural question arises how one can certify any multiphoton experiment when both the network and the number of indistinguishable photons are enlarged such that the experiments are no longer simulable on classical computers. We introduce a one-sided device-independent photonic indistinguishability witness to do just that. This witness relies on a computationally efficient two-mode correlator that can be measured using two photon-number-resolving detectors. The witness is one-sided device-independent since any experimental deviation from the ideal measurement will automatically reduce the lower bound of the witness. A three-photon proof-of-principle experiment was conducted to verify the experimental feasibility of the proposed witness.

Finally, we address the question of why a pure quantum state can show thermalized behavior in a closed system. We simulate the time evolution of both a non-interacting Bose-Hubbard superfluid and random long-range Hamiltonians on a photonic processor. We show that even though the overall state is pure, locally it becomes a mixed state. We attribute this to entanglement correlations with the unobserved 'universe', the universe is the remaining part of the processor. Moreover, we certify this hypothesis to be correct by exploiting reversibility and undoing the entanglement correlations.

In short, we have realized a top-of-the-line multiphoton source. With this setup, we have performed the first-ever quantum experiments with a promising new integrated photonic platform. These experiments have solved a four-decade-old problem of mesoscopic physics, introduced a one-sided device-independent indistinguishability witness and finally proved that entanglement causes pure quantum states to locally thermalize.

\subsection{Outlook}

In this thesis, we have explored potential candidates to implement large optical networks for quantum optics, limitations of multiphoton sources and finally demonstrated a few quantum experiments on integrated photonics. There are numerous extensions possible within of these three themes.

Firstly, we will start with possible directions in the field of photon sources. Secondly, we then proceed with a discussion of the possible modes. Finally, we end with a discussion on possible experiments that can be performed with the type of hardware that can be realized in the foreseeable future. 


\subsubsection{Improving the multiphoton source}

There are different types of single-photon sources that can be used to scale up to larger systems. In the long term, it is convenient to integrate multiphoton sources with the optical networks, since that would both enable scalability and guarantee phase stability. There are a few options for how this can be realized. In the short term, there are other relatively straightforward improvements that can be made to the current source.

First and foremost, an interesting upgrade to the current multiphoton source is to convert it into a picosecond multiphoton source. This has three distinct advantages. The first advantage is that picosecond pulses allow for spatial mode cleaning, e.g. correcting beam astigmatism, of the laser with a short single-mode fiber and can consequently improve the fiber coupling of the generated photons. This will help to increase the heralding efficiency above $50 \%$.

The second advantage of a picosecond source is that the smaller spectral width of the photons enables a higher fidelity of the implemented matrix settings. This can be understood directly from the fact that the optical processor implements a wavelengthdependent transfer matrix of the photons. A smaller spectral width will make the matrix more alike for the spectral extremes of the light. This effect is expected to be small, but it can become necessary for significantly larger systems. For example, it is known that the matrix amplitude fidelity increases from 0.965 to 0.98 when the spectral with is decreased from 25 to $10 \mathrm{~nm}$. This improvement seems misleadingly small, but the fidelity for a three-photons experiment increases from $0.965^{6}=0.808$ to $0.98^{6}=0.886$, a significant effect.

The third and final advantage is that longer pulses will allow for time-bin experiments. Currently, this is not possible as any integrated network has dispersion and hence femtosecond pulses will quickly accumulate spectral chirp. This chirp makes interference between early and late photons in a time-bin experiment impossible as the photons will quickly become too distinguishable. Longer and spectrally narrower photons decrease the effects of dispersion and will be more robust against small path length differences.

An optimal picosecond ppKTP source requires a longer nonlinear crystal since that is necessary to keep the JSI factorizable. Using the methods of Chapter 3, we find that an optimal ppKTP source which is pumped with $775 \mathrm{~nm}$ and a linewidth of $1 \mathrm{~nm}$ consists of a $10 \mathrm{~mm}$ ppKTP crystal. The spectral purity of the picosecond source is practically identical to the current setup (81\%). The spectral purity can be improved by spectral filtering with a $5 \mathrm{~nm}$ bandpass filter, but this comes at the cost of about $10 \%$ losses. The associated source quality increases from $\alpha_{0}=0.81$ to $\alpha_{f}=0.88$, indicating that filtering is beneficial from a computational complexity point of view [280].

A better option to improve the setup is to switch to apodized poled crystals, which are known to be optimal for multiphoton interference [280]. These crystals can achieve high spectral purities without the need of filtering and they are also suitable for picosecond sources. Some examples of SPDC sources based on apodized poled crystals are already reported in literature [119, 121-123]. A $29 \mathrm{~mm}$ picosecond aperiodic KTP source is reported to have a purity of 0.953 and a heralding efficiency of 0.65 (after correcting for 
known detection efficiency and optical losses) [122].

A final and smaller possible upgrade of the current setup could be to deal with detector blinding. When an output distribution is sampled, then the most likely output modes tend to be under-sampled because the detector dead time is longer than the pulse repetition rate, which are $50 \mathrm{~ns}$ and $12.5 \mathrm{~ns}$, respectively. Currently, this problem is mitigated by reducing the pump power sufficiently so that the average time between two photons arriving at the detector is (much) larger than the detector dead time. However, a pulse picker can be used to reduce the pulse repetition rate of the laser such that the consecutive laser pulses are more than $50 \mathrm{~ns}$ apart.

Integration of multiphoton sources into integrated photonic networks is an obvious route to pursue. There are various ways to achieve this. Firstly, it is possible to integrate SPDC sources in waveguides. A notable example are ppln waveguide sources [141], which unfortunately creates nondegenerate photons and aperiodically poled ppln waveguides are not (yet) reported in literature.

A different approach is to use spontaneous four-wave mixing (SFWM). SFWM can be realized with a high Q-factor ring resonator and a $\chi^{(3)}$-nonlinearity, which is feasible in integrated photonics. The high Q-factor ensures a high spectral purity, up to 0.99 has been reported [40]. Furthermore, the losses are insignificant when implemented in SiN [281]. Unfortunately, a third-order nonlinearity is inherently weak, resulting in low generation rates and the process might be polluted by other weak nonlinear effects [281], although some techniques have been proposed to suppress this [282, 283]

An entirely different approach would be to use quantum dots. Quantum dot sources allow for high generation rates of pure and single photons with a long coherence time $[35,284]$. Quantum dot sources are inherently suitable for time-bin experiments since otherwise a spatio-temporal demultiplexer should be constructed, which is feasible but nontrivial [285]. However, this limitation can be circumvented when quantum dots become reproducible. There are hints that this might become possible [286].

\subsubsection{Future integrated optical networks}

A second, major piece of equipment is the integrated photonic quantum processor. For future experiments, larger low-loss and fully reconfigurable networks will be needed. This is not just a matter of enlarging the current chip. There are several limitations to the current processor. An example is the use of thermo-optical switches. Notwithstanding the current success of these thermo-optical switches, the energy dissipation will continue to increase to a point when either the power consumption or cooling capacities have reached their maximum. This problem can be circumvented by adopting other switching methods. An example are stress-optical modulators using PZT [287, 288] or optomechanics [289, 290]. Other improvements to the integrated processor can be the integration of single-photon sources and detectors [291, 292]. The latter requires the processor to operate at cryogenic temperatures.

Thus far, the discussion was restricted to similar architectures to the one presented in this thesis. Other architectures have their use too. For example, circulating meshes 
can allow to implement a more natural physical simulation of scattering physics. When scaled up, it could be used to further investigate other well-known physical phenomena such as Anderson localization in 3D [293] or to study multiphoton scattering physics such as universal conductance flutuations. Another different direction is to include optical nonlinearities, e.g. via ring resonators or atom-photon interaction, in the photonic processors [294]. Finally, integrated photonics can also be used to control trapped ions [27] or to utilize the large optical bandwidth for machine learning [167]. 



\section{Bibliography}

[1] J. Al-Khalili and J. McFadden, You're powered by quantum mechanics. no, really..., www.theguardian.com (2021). The Observer, https://www.theguardian.com/science/2014/oct/26/ youre-powered-by-quantum-mechanics-biology.

[2] Y. Manin, Comutable and uncomputable, Sov. Radio (1980).

[3] R. P. Feynman, Simulating physics with computers, Int J Theor Phys, 21, 467-488 (1982).

[4] P. Shor, Algorithms for quantum computation: Discrete logarithms and factoring, in Proceedings 35th Annual Symposium on Foundations of Computer Science, pp. 124-134 (1994).

[5] L. K. Grover, A fast quantum mechanical algorithm for database search, in Proceedings of the Twenty-Eighth Annual ACM Symposium on Theory of Computing - STOC '96, pp. 212-219, ACM Press, Philadelphia, Pennsylvania, United States (1996).

[6] M. Nielsen and I. Chuang, Quantum Computation and Quantum Information: 10th Anniversary Edition, Cambridge University Press (2010).

[7] C. Outeiral, M. Strahm, J. Shi, G. M. Morris, S. C. Benjamin, and C. M. Deane, The prospects of quantum computing in computational molecular biology, WIREs Computational Molecular Science, 11, e1481 (2021).

[8] J. Biamonte, P. Wittek, N. Pancotti, P. Rebentrost, N. Wiebe, and S. Lloyd, Quantum machine learning, Nature, 549, 195-202 (2017).

[9] S. Wehner, D. Elkouss, and R. Hanson, Quantum internet: A vision for the road ahead, Science, 362, eaam9288 (2018).

[10] F. Arute, K. Arya, R. Babbush, D. Bacon, J. C. Bardin, et al., Quantum supremacy using a programmable superconducting processor, Nature, 574, 505-510 (2019).

[11] H.-S. Zhong, Y.-H. Deng, J. Qin, H. Wang, M.-C. Chen, et al., Phase-Programmable Gaussian Boson Sampling Using Stimulated Squeezed Light, Phys. Rev. Lett., 127, 180502 (2021).

[12] Y. Wu, W.-S. Bao, S. Cao, F. Chen, M.-C. Chen, et al., Strong Quantum Computational Advantage Using a Superconducting Quantum Processor, Phys. Rev. Lett., 127, 180501 (2021).

[13] D. Stilck França and R. García-Patrón, Limitations of optimization algorithms on noisy quantum devices, Nat. Phys., 17, 1-7 (2021).

[14] P. Kaye, R. Laflamme, and M. Mosca, An Introduction to Quantum Computing, Oxford University Press (2007).

[15] S. Aaronson and A. Arkhipov, The Computational Complexity of Linear Optics, Theory Comput., 9, 143-252 (2013).

[16] S. Boixo, S. V. Isakov, V. N. Smelyanskiy, R. Babbush, N. Ding, Z. Jiang, M. J. Bremner, J. M. Martinis, and H. Neven, Characterizing quantum supremacy in near-term devices, Nat. Phys., 14, 595-600 (2018). 
[17] B. J. Metcalf, N. Thomas-Peter, J. B. Spring, D. Kundys, M. A. Broome, P. C. Humphreys, X.-M. Jin, M. Barbieri, W. Steven Kolthammer, J. C. Gates, B. J. Smith, N. K. Langford, P. G. R. Smith, and I. A. Walmsley, Multiphoton quantum interference in a multiport integrated photonic device, Nat. Commun., 4, 1356 (2013).

[18] M. A. Broome, A. Fedrizzi, S. Rahimi-Keshari, J. Dove, S. Aaronson, T. C. Ralph, and A. G. White, Photonic Boson Sampling in a Tunable Circuit, Science, 339, 794-798 (2013).

[19] J. B. Spring, B. J. Metcalf, P. C. Humphreys, W. S. Kolthammer, X.-M. Jin, M. Barbieri, A. Datta, N. Thomas-Peter, N. K. Langford, D. Kundys, J. C. Gates, B. J. Smith, P. G. R. Smith, and I. A. Walmsley, Boson Sampling on a Photonic Chip, Science, 339, 798-801 (2013).

[20] M. Tillmann, B. Dakić, R. Heilmann, S. Nolte, A. Szameit, and P. Walther, Experimental boson sampling, Nat. Photonics, 7, 540- (2013).

[21] A. Crespi, R. Osellame, R. Ramponi, D. J. Brod, E. F. Galvão, N. Spagnolo, C. Vitelli, E. Maiorino, P. Mataloni, and F. Sciarrino, Integrated multimode interferometers with arbitrary designs for photonic boson sampling, Nat. Photonics, 7, 545 (2013).

[22] N. Spagnolo, C. Vitelli, M. Bentivegna, D. J. Brod, A. Crespi, F. Flamini, S. Giacomini, G. Milani, R. Ramponi, P. Mataloni, R. Osellame, E. F. Galvao, and F. Sciarrino, Experimental validation of photonic boson sampling, Nat. Photonics, 8, 615-621 (2014).

[23] H. Wang, Y. He, Y.-H. Li, Z.-E. Su, B. Li, H.-L. Huang, X. Ding, M.-C. Chen, C. Liu, J. Qin, J.-P. Li, Y.-M. He, C. Schneider, M. Kamp, C.-Z. Peng, S. Höfling, C.Y. Lu, and J.-W. Pan, High-efficiency multiphoton boson sampling, Nat. Photonics, 11, 361 (2017).

[24] H. Wang, J. Qin, X. Ding, M.-C. Chen, S. Chen, X. You, Y.-M. He, X. Jiang, L. You, Z. Wang, C. Schneider, J. J. Renema, S. Höfling, C.-Y. Lu, and J.-W. Pan, Boson Sampling with 20 Input Photons and a 60-Mode Interferometer in a \$1\{0\}\{14\}\$-Dimensional Hilbert Space, Phys. Rev. Lett., 123, 250503 (2019).

[25] H.-S. Zhong, H. Wang, Y.-H. Deng, M.-C. Chen, L.-C. Peng, et al., Quantum computational advantage using photons, Science (2020).

[26] B. J. Shastri, A. N. Tait, T. Ferreira de Lima, W. H. P. Pernice, H. Bhaskaran, C. D. Wright, and P. R. Prucnal, Photonics for artificial intelligence and neuromorphic computing, Nat. Photonics, 15, 102-114 (2021).

[27] K. K. Mehta, C. Zhang, M. Malinowski, T.-L. Nguyen, M. Stadler, and J. P. Home, Integrated optical multi-ion quantum logic, Nature, 586, 533-537 (2020).

[28] M. D. Eisaman, J. Fan, A. Migdall, and S. V. Polyakov, Invited Review Article: Single-photon sources and detectors, Rev. Sci. Instrum., 82, 071101 (2011).

[29] P. G. Kwiat, K. Mattle, H. Weinfurter, A. Zeilinger, A. V. Sergienko, and Y. Shih, New High-Intensity Source of Polarization-Entangled Photon Pairs, Phys. Rev. Lett., 75, 4337-4341 (1995). 
[30] M. M. Weston, H. M. Chrzanowski, S. Wollmann, A. Boston, J. Ho, L. K. Shalm, V. B. Verma, M. S. Allman, S. W. Nam, R. B. Patel, S. Slussarenko, and G. J. Pryde, Efficient and pure femtosecond-pulse-length source of polarization-entangled photons, Opt. Express, 24, 10869-10879 (2016).

[31] F. Kaneda, B. G. Christensen, J. J. Wong, H. S. Park, K. T. McCusker, and P. G. Kwiat, Time-multiplexed heralded single-photon source, Optica, 2, 1010-1013 (2015).

[32] A. P. Lund, A. Laing, S. Rahimi-Keshari, T. Rudolph, J. L. O'Brien, and T. C. Ralph, Boson Sampling from a Gaussian State, Phys. Rev. Lett., 113, 100502 (2014).

[33] T. Hummel, C. Ouellet-Plamondon, E. Ugur, I. Kulkova, T. Lund-Hansen, M. A. Broome, R. Uppu, and P. Lodahl, Efficient demultiplexed single-photon source with a quantum dot coupled to a nanophotonic waveguide, Appl. Phys. Lett., 115, 021102 (2019).

[34] C. Antón, J. C. Loredo, G. Coppola, H. Ollivier, N. Viggianiello, A. Harouri, N. Somaschi, A. Crespi, I. Sagnes, A. Lemaître, L. Lanco, R. Osellame, F. Sciarrino, and P. Senellart, Interfacing scalable photonic platforms: Solid-state based multiphoton interference in a reconfigurable glass chip, Optica, 6, 1471-1477 (2019).

[35] P. Senellart, G. Solomon, and A. White, High-performance semiconductor quantum-dot single-photon sources, Nat. Nanotechnol., 12, 1026-1039 (2017).

[36] H.-S. Zhong, Y. Li, W. Li, L.-C. Peng, Z.-E. Su, Y. Hu, Y.-M. He, X. Ding, W. Zhang, H. Li, L. Zhang, Z. Wang, L. You, X.-L. Wang, X. Jiang, L. Li, Y.-A. Chen, N.-L. Liu, C.-Y. Lu, and J.-W. Pan, 12-Photon Entanglement and Scalable Scattershot Boson Sampling with Optimal Entangled-Photon Pairs from Parametric Down-Conversion, Phys. Rev. Lett., 121, 250505 (2018).

[37] H. Wang, Y.-M. He, T.-H. Chung, H. Hu, Y. Yu, et al., Towards optimal singlephoton sources from polarized microcavities, Nat. Photonics, 13, 770-775 (2019).

[38] R. Uppu, F. T. Pedersen, Y. Wang, C. T. Olesen, C. Papon, X. Zhou, L. Midolo, S. Scholz, A. D. Wieck, A. Ludwig, and P. Lodahl, Scalable integrated singlephoton source, Sci. Adv., 6, eabc8268 (2020).

[39] L. J. Wang, C. K. Hong, and S. R. Friberg, Generation of correlated photons via four-wave mixing in optical fibres, J. Opt. B: Quantum Semiclassical Opt., 3, 346-352 (2001).

[40] S. Paesani, M. Borghi, S. Signorini, A. Maïnos, L. Pavesi, and A. Laing, Near-ideal spontaneous photon sources in silicon quantum photonics, Nat. Commun., 11, 2505 (2020).

[41] C. Taballione, Integrated Programmable Waveguide Circuits for Classical and Quantum Photonic Processing, Ph.D. thesis, University of Twente, Enschede (2019).

[42] D. E. Browne and T. Rudolph, Resource-Efficient Linear Optical Quantum Computation, Phys. Rev. Lett., 95, 010501 (2005).

[43] S. Bartolucci, P. Birchall, H. Bombin, H. Cable, C. Dawson, M. Gimeno-Segovia, 
E. Johnston, K. Kieling, N. Nickerson, M. Pant, F. Pastawski, T. Rudolph, and

C. Sparrow, Fusion-based quantum computation, arXiv: 2101.09310 (2021).

[44] A. W. Elshaari, W. Pernice, K. Srinivasan, O. Benson, and V. Zwiller, Hybrid integrated quantum photonic circuits, Nat. Photonics, 14, 285-298 (2020).

[45] J. Wang, F. Sciarrino, A. Laing, and M. G. Thompson, Integrated photonic quantum technologies, Nat. Photonics, 14, 273-284 (2020).

[46] C. Taballione, R. van der Meer, H. J. Snijders, P. Hooijschuur, J. P. Epping, M. de Goede, B. Kassenberg, P. Venderbosch, C. Toebes, H. van den Vlekkert, P. W. H. Pinkse, and J. J. Renema, A universal fully reconfigurable 12-mode quantum photonic processor, Mater. Quantum. Technol., 1, 035002 (2021).

[47] S. Rotter and S. Gigan, Light fields in complex media: Mesoscopic scattering meets wave control, Rev. Mod. Phys., 89, 015005 (2017).

[48] C. W. J. Beenakker, Applications of random matrix theory to condensed matter and optical physics, arXiv:0904.1432 (2009).

[49] O. Dorokhov, Transmission coefficient and the localization length of an electron in $N$ bound disordered chains, JETP Lett., 36 (1982).

[50] P. A. Mello, P. Pereyra, and N. Kumar, Macroscopic approach to multichannel disordered conductors, Ann. Phys. (NY), 181, 290-317 (1988).

[51] H. Yu, T. R. Hillman, W. Choi, J. O. Lee, M. S. Feld, R. R. Dasari, and Y. Park, Measuring Large Optical Transmission Matrices of Disordered Media, Phys. Rev. Lett., 111, 153902 (2013).

[52] A. Goetschy and A. D. Stone, Filtering Random Matrices: The Effect of Incomplete Channel Control in Multiple Scattering, Phys. Rev. Lett., 111, 063901 (2013).

[53] S. M. Popoff, A. Goetschy, S. F. Liew, A. D. Stone, and H. Cao, Coherent Control of Total Transmission of Light through Disordered Media, Phys. Rev. Lett., 112, 133903 (2014).

[54] M. Bentivegna, N. Spagnolo, C. Vitelli, D. J. Brod, A. Crespi, F. Flamini, R. Ramponi, P. Mataloni, R. Osellame, E. F. Galvão, and F. Sciarrino, Bayesian approach to Boson sampling validation, Int. J. Quantum Inf., 12, 1560028 (2014).

[55] M. Walschaers, J. Kuipers, J.-D. Urbina, K. Mayer, M. C. Tichy, K. Richter, and A. Buchleitner, Statistical benchmark for BosonSampling, New J. Phys., 18, 032001 (2016).

[56] D. J. Brod, E. F. Galvão, N. Viggianiello, F. Flamini, N. Spagnolo, and F. Sciarrino, Witnessing Genuine Multiphoton Indistinguishability, Phys. Rev. Lett., 122, 063602 (2019).

[57] S. Popescu, A. J. Short, and A. Winter, Entanglement and the foundations of statistical mechanics, Nat. Phys., 2, 754-758 (2006).

[58] A. M. Kaufman, M. E. Tai, A. Lukin, M. Rispoli, R. Schittko, P. M. Preiss, and M. Greiner, Quantum thermalization through entanglement in an isolated manybody system, Science, 353, 794-800 (2016). 
[59] J. J. Renema, V. Shchesnovich, and R. Garcia-Patron, Classical simulability of noisy boson sampling, arXiv:1809.01953 (2018).

[60] N. Walk, Fidelity certication for photonic states with non-ideal detectors (2021). Fidelity certication for photonic states with non-ideal detectors (unpublished).

[61] H. J. Ryser, Combinatorial Mathematics (Carus Mathematical Monographs No. 14), Mathematical Assn of Amer (1963).

[62] J. Wu, Y. Liu, B. Zhang, X. Jin, Y. Wang, H. Wang, and X. Yang, A Benchmark Test of Boson Sampling on Tianhe-2 Supercomputer, Natl. Sci. Rev., 5, 715-720 (2018).

[63] J. C. Loredo, M. A. Broome, P. Hilaire, O. Gazzano, I. Sagnes, A. Lemaitre, M. P. Almeida, P. Senellart, and A. G. White, Boson Sampling with Single-Photon Fock States from a Bright Solid-State Source, Phys. Rev. Lett., 118, 130503 (2017).

[64] Y. He, X. Ding, Z.-E. Su, H.-L. Huang, J. Qin, C. Wang, S. Unsleber, C. Chen, H. Wang, Y.-M. He, X.-L. Wang, W.-J. Zhang, S.-J. Chen, C. Schneider, M. Kamp, L.-X. You, Z. Wang, S. Höfling, C.-Y. Lu, and J.-W. Pan, Time-Bin-Encoded Boson Sampling with a Single-Photon Device, Phys. Rev. Lett., 118, 190501 (2017).

[65] C. S. Hamilton, R. Kruse, L. Sansoni, S. Barkhofen, C. Silberhorn, and I. Jex, Gaussian Boson Sampling, Phys. Rev. Lett., 119, 170501 (2017). 1612 . 01199.

[66] J. J. Renema, A. Menssen, W. R. Clements, G. Triginer, W. S. Kolthammer, and I. A. Walmsley, Efficient Classical Algorithm for Boson Sampling with Partially Distinguishable Photons, Phys. Rev. Lett., 120, 220502 (2018).

[67] R. García-Patrón, J. J. Renema, and V. Shchesnovich, Simulating boson sampling in lossy architectures, Quantum, 3, 169 (2019).

[68] D. J. Brod and M. Oszmaniec, Classical simulation of linear optics subject to nonuniform losses, Quantum, 4, 267 (2020). 1906.06696.

[69] J. J. Renema, Simulability of partially distinguishable superposition and Gaussian boson sampling, Phys. Rev. A, 101, 063840 (2020).

[70] B. Villalonga, M. Y. Niu, L. Li, H. Neven, J. C. Platt, V. N. Smelyanskiy, and S. Boixo, Efficient approximation of experimental gaussian boson sampling, Arxiv:2109.11525 (2021).

[71] J. Shi and T. Byrnes, Gaussian boson sampling with partial distinguishability, arXiv:2105.09583 (2021).

[72] F. Pan and P. Zhang, Simulating the Sycamore quantum supremacy circuits, arXiv:2103.03074 (2021).

[73] J. F. F. Bulmer, B. A. Bell, R. S. Chadwick, A. E. Jones, D. Moise, A. Rigazzi, J. Thorbecke, U.-U. Haus, T. Van Vaerenbergh, R. B. Patel, I. A. Walmsley, and A. Laing, The Boundary for Quantum Advantage in Gaussian Boson Sampling, arXiv:2108.01622 (2021).

[74] A. S. Popova and A. N. Rubtsov, Cracking the Quantum Advantage threshold for Gaussian Boson Sampling, arXiv:2106.01445 (2021). 
[75] V. S. Shchesnovich, On the classical complexity of sampling from quantum interference of indistinguishable bosons, Int. J. Quantum Inform., 18, 2050044 (2020).

[76] A. P. Mosk, A. Lagendijk, G. Lerosey, and M. Fink, Controlling waves in space and time for imaging and focusing in complex media, Nat. Photonics, 6, 283-292 (2012).

[77] R. Barak and Y. Ben-Aryeh, Quantum fast Fourier transform and quantum computation by linear optics, J. Opt. Soc. Am. B, 24, 231 (2007).

[78] A. Crespi, R. Osellame, R. Ramponi, M. Bentivegna, F. Flamini, N. Spagnolo, N. Viggianiello, L. Innocenti, P. Mataloni, and F. Sciarrino, Suppression law of quantum states in a 3D photonic fast Fourier transform chip, Nat. Commun., 7, 10469 (2016).

[79] V. L. Pastor, J. Lundeen, J. Lundeen, and F. Marquardt, Arbitrary optical wave evolution with Fourier transforms and phase masks, Opt. Express, 29, 3844138450 (2021).

[80] I. M. Vellekoop and A. P. Mosk, Focusing coherent light through opaque strongly scattering media, Opt. Lett., 32, 2309-2311 (2007).

[81] J. Goodman, Speckle Phenomena in Optics: Theory and Applications, Roberts \& Company (2007).

[82] H. Di Lorenzo Pires, J. Woudenberg, and M. P. van Exter, Statistical properties of two-photon speckles, Phys. Rev. A, 85, 033807 (2012).

[83] H. Defienne, M. Barbieri, B. Chalopin, B. Chatel, I. A. Walmsley, B. J. Smith, and S. Gigan, Nonclassical light manipulation in a multiple-scattering medium, Opt. Lett., 39, 6090 (2014).

[84] T. J. Huisman, S. R. Huisman, A. P. Mosk, and P. W. H. Pinkse, Controlling singlephoton Fock-state propagation through opaque scattering media, Appl. Phys. B, 116, 603-607 (2014).

[85] T. A. W. Wolterink, R. Uppu, G. Ctistis, W. L. Vos, K.-J. Boller, and P. W. H. Pinkse, Programmable two-photon quantum interference in $10^{3}$ channels in opaque scattering media, Phys. Rev. A, 93, 053817 (2016).

[86] W. H. Peeters, J. J. D. Moerman, and M. P. van Exter, Observation of Two-Photon Speckle Patterns, Phys. Rev. Lett., 104, 173601 (2010).

[87] D. J. Brod, E. F. Galvão, A. Crespi, R. Osellame, N. Spagnolo, and F. Sciarrino, Photonic implementation of boson sampling: A review, Adv. Photonics, 1, 034001 (2019).

[88] S. Scheel, Permanents in linear optical networks, arXiv:0406127 (2004).

[89] A. Arkhipov and G. Kuperberg, The bosonic birthday paradox, Geom. Topol. Monogr., 18, 1-7 (2012).

[90] V. Bentkus, A Lyapunov-type Bound in $R^{d}$, Theory Probab. Appl., 49, 311-323 (2005).

[91] M. W. Meckes, Some Results on Random Circulant Matrices, Institute of Mathematical Statistics (2009). 
[92] C. Mastrodonato and R. Tumulka, Elementary Proof for Asymptotics of Large Haar-Distributed Unitary Matrices, Lett. Math. Phys., 82, 51-59 (2007).

[93] D. Petz and J. Réffy, On asymptotics of large Haar distributed unitary matrices, Period. Math. Hung., 49, 103-117 (2005).

[94] D. Petz and J. Réffy, Large deviation for the empirical eigenvalue density of truncated Haar unitary matrices, Probab. Theory Relat. Fields, 133, 175-189 (2005).

[95] J. Réffy, Asymptotics of Random Unitaries, Ph.D. thesis, Budapest University of Technology and Economics, Budapest (2005).

[96] A. E. Lita, A. J. Miller, and S. W. Nam, Counting near-infrared single-photons with 95\% efficiency, Opt. Express, 16, 3032-3040 (2008).

[97] D. V. Reddy, D. V. Reddy, A. E. Lita, S. W. Nam, R. P. Mirin, and V. B. Verma, Achieving 98\% system efficiency at $1550 \mathrm{~nm}$ in superconducting nanowire single photon detectors, in Rochester Conference on Coherence and Quantum Optics (CQO-11) (2019), Paper W2B.2, p. W2B.2, Optical Society of America (2019).

[98] S. Doerner, A. Kuzmin, S. Wuensch, I. Charaev, F. Boes, T. Zwick, and M. Siegel, Frequency-multiplexed bias and readout of a 16-pixel superconducting nanowire single-photon detector array, Appl. Phys. Lett., 111, 032603 (2017).

[99] A. Gaggero, F. Martini, F. Mattioli, F. Chiarello, R. Cernansky, A. Politi, and R. Leoni, Amplitude-multiplexed readout of single photon detectors based on superconducting nanowires, Optica, 6, 823-828 (2019).

[100] S. Miki, T. Yamashita, Z. Wang, and H. Terai, A 64-pixel NbTiN superconducting nanowire single-photon detector array for spatially resolved photon detection, Opt. Express, 22, 7811-7820 (2014).

[101] S. Miyajima, M. Yabuno, S. Miki, T. Yamashita, and H. Terai, High-time-resolved 64-channel single-flux quantum-based address encoder integrated with a multi-pixel superconducting nanowire single-photon detector, Opt. Express, 26, 29045-29054 (2018).

[102] A. K. Sinclair, E. Schroeder, D. Zhu, M. Colangelo, J. Glasby, P. D. Mauskopf, H. Mani, and K. K. Berggren, Demonstration of Microwave Multiplexed Readout of DC-Biased Superconducting Nanowire Detectors, IEEE Trans. Appl. Supercond., 29, 1-4 (2019).

[103] Q. Zhao, A. McCaughan, F. Bellei, F. Najafi, D. De Fazio, A. Dane, Y. Ivry, and K. K. Berggren, Superconducting-nanowire single-photon-detector linear array, Appl. Phys. Lett., 103, 142602 (2013).

[104] Q.-Y. Zhao, D. Zhu, N. Calandri, A. E. Dane, A. N. McCaughan, F. Bellei, H.-Z. Wang, D. F. Santavicca, and K. K. Berggren, Single-photon imager based on a superconducting nanowire delay line, Nat. Photonics, 11, 247-251 (2017).

[105] D. Zhu, Q.-Y. Zhao, H. Choi, T.-J. Lu, A. E. Dane, D. Englund, and K. K. Berggren, A scalable multi-photon coincidence detector based on superconducting nanowires, Nat. Nanotechnol., 13, 596-601 (2018).

[106] J. P. Allmaras, E. E. Wollman, A. D. Beyer, R. M. Briggs, B. A. Korzh, B. Bumble, 
and M. D. Shaw, Demonstration of a Thermally Coupled Row-Column SNSPD Imaging Array, Nano Lett., 20, 2163-2168 (2020).

[107] E. E. Wollman, E. E. Wollman, V. B. Verma, V. B. Verma, A. E. Lita, W. H. Farr, M. D. Shaw, R. P. Mirin, and S. W. Nam, Kilopixel array of superconducting nanowire single-photon detectors, Opt. Express, 27, 35279-35289 (2019).

[108] M. S. Allman, V. B. Verma, M. Stevens, T. Gerrits, R. D. Horansky, A. E. Lita, F. Marsili, A. Beyer, M. D. Shaw, D. Kumor, R. Mirin, and S. W. Nam, A nearinfrared 64-pixel superconducting nanowire single photon detector array with integrated multiplexed readout, Appl. Phys. Lett., 106, 192601 (2015).

[109] A. W. Harrow and A. Montanaro, Quantum computational supremacy, Nature, 549, 203-209 (2017).

[110] S. Paesani, Y. Ding, R. Santagati, L. Chakhmakhchyan, C. Vigliar, K. Rottwitt, L. K. Oxenløwe, J. Wang, M. G. Thompson, and A. Laing, Generation and sampling of quantum states of light in a silicon chip, arXiv:1812.03158 (2018).

[111] L. Latmiral, N. Spagnolo, and F. Sciarrino, Towards quantum supremacy with lossy scattershot boson sampling, New J. Phys., 18, 113008 (2016).

[112] P. P. Rohde and T. C. Ralph, Error tolerance of the boson-sampling model for linear optics quantum computing, Phys. Rev. A, 85, 022332 (2012).

[113] E. Meyer-Scott, N. Montaut, J. Tiedau, L. Sansoni, H. Herrmann, T. J. Bartley, and C. Silberhorn, Limits on the heralding efficiencies and spectral purities of spectrally filtered single photons from photon-pair sources, Phys. Rev. A, 95, 061803 (2017).

[114] R. S. Bennink, Optimal collinear Gaussian beams for spontaneous parametric down-conversion, Phys. Rev. A, 81, 053805 (2010).

[115] F. Graffitti, J. Kelly-Massicotte, A. Fedrizzi, and A. M. Brańczyk, Design considerations for high-purity heralded single-photon sources, Phys. Rev. A, 98, 053811 (2018).

[116] S. Slussarenko, M. M. Weston, H. M. Chrzanowski, L. K. Shalm, V. B. Verma, S. W. Nam, and G. J. Pryde, Unconditional violation of the shot-noise limit in photonic quantum metrology, Nat. Photonics, 11, 700 (2017).

[117] A. Neville, C. Sparrow, R. Clifford, E. Johnston, P. A. Birchall, A. Montanaro, and A. Laing, Classical boson sampling algorithms with superior performance to near-term experiments, Nat. Phys., 13, 1153-1157 (2017).

[118] T. Kim, M. Fiorentino, and F. N. C. Wong, Phase-stable source of polarizationentangled photons using a polarization Sagnac interferometer, Phys. Rev. A, 73, 012316 (2006).

[119] A. M. Brańczyk, A. Fedrizzi, T. M. Stace, T. C. Ralph, and A. G. White, Engineered optical nonlinearity for quantum light sources, Opt. Express, 19, 55-65 (2011).

[120] P. B. Dixon, J. H. Shapiro, and F. N. C. Wong, Spectral engineering by Gaussian phase-matching for quantum photonics, Opt. Express, 21, 5879-5890 (2013).

[121] C. Chen, C. Bo, M. Y. Niu, F. Xu, Z. Zhang, J. H. Shapiro, and F. N. C. Wong, 
Efficient generation and characterization of spectrally factorable biphotons, Opt. Express, 25, 7300-7312 (2017).

[122] F. Graffitti, P. Barrow, M. Proietti, D. Kundys, and A. Fedrizzi, Independent highpurity photons created in domain-engineered crystals, Optica, 5, 514 (2018).

[123] A. Pickston, F. Graffitti, P. Barrow, C. L. Morrison, J. Ho, A. M. Brańczyk, and A. Fedrizzi, Optimised domain-engineered crystals for pure telecom photon sources, Opt. Express, 29, 6991-7002 (2021).

[124] P. J. Mosley, J. S. Lundeen, B. J. Smith, P. Wasylczyk, A. B. U'Ren, C. Silberhorn, and I. A. Walmsley, Heralded Generation of Ultrafast Single Photons in Pure Quantum States, Phys. Rev. Lett., 100, 133601 (2008).

[125] D. Rosenberg, A. E. Lita, A. J. Miller, and S. W. Nam, Noise-free high-efficiency photon-number-resolving detectors, Phys. Rev. A, 71, 061803 (2005).

[126] W. P. Grice and I. A. Walmsley, Spectral information and distinguishability in type-II down-conversion with a broadband pump, Phys. Rev. A, 56, 1627-1634 (1997).

[127] A.B, U'Ren, and C. Silberhorn, and R. Erdmann, and K. Banaszek, and W.P. Grice, and I.A. Walmsley, and M.G. Raymer, Generation of Pure-State SinglePhoton Wavepackets by Conditional Preparation Based on Spontaneous Parametric Downconversion, Las. Phys., 15, 146-161 (2005).

[128] Z.-Y. J. Ou, Multi-Photon Quantum Interference, Springer, New York (2017).

[129] C. K. Law, I. A. Walmsley, and J. H. Eberly, Continuous Frequency Entanglement: Effective Finite Hilbert Space and Entropy Control, Phys. Rev. Lett., 84, 5304-5307 (2000).

[130] J. H. Eberly, Schmidt analysis of pure-state entanglement, Laser Phys., 16, 921-926 (2006).

[131] A. Christ, K. Laiho, A. Eckstein, K. N. Cassemiro, and C. Silberhorn, Probing multimode squeezing with correlation functions, New J. Phys., 13, 033027 (2011).

[132] M. Oszmaniec and D. J. Brod, Classical simulation of photonic linear optics with lost particles, New J. Phys., 20, 092002 (2018).

[133] C. K. Hong, Z. Y. Ou, and L. Mandel, Measurement of subpicosecond time intervals between two photons by interference, Phys. Rev. Lett., 59, 2044-2046 (1987).

[134] T. E. Keller and M. H. Rubin, Theory of two-photon entanglement for spontaneous parametric down-conversion driven by a narrow pump pulse, Phys. Rev. A, 56, 1534-1541 (1997).

[135] W. P. Grice, A. B. U'Ren, and I. A. Walmsley, Eliminating frequency and space-time correlations in multiphoton states, Phys. Rev. A, 64, 063815 (2001).

[136] T. Gerrits, M. J. Stevens, B. Baek, B. Calkins, A. Lita, S. Glancy, E. Knill, S. W. Nam, R. P. Mirin, R. H. Hadfield, R. S. Bennink, W. P. Grice, S. Dorenbos, T. Zijlstra, T. Klapwijk, and V. Zwiller, Generation of degenerate, factorizable, pulsed squeezed light at telecom wavelengths, Opt. Express, 19, 24434-24447 (2011). 
[137] P. J. Mosley, J. S. Lundeen, B. J. Smith, and I. A. Walmsley, Conditional preparation of single photons using parametric downconversion: A recipe for purity, New $\mathrm{J}$. Phys., 10, 093011 (2008).

[138] F. Marsili, V. B. Verma, J. A. Stern, S. Harrington, T. Gerrits, A. E. Lita, I. Vayshenker, B. baek, M. D. Shaw, R. P. Mirin, and S. W. Nam, Detecting single infrared photons with 93\% system efficiency, Nat. Photonics, 7, 210-214 (2013).

[139] D. V. Reddy, R. R. Nerem, S. W. Nam, R. P. Mirin, and V. B. Verma, Superconducting nanowire single-photon detectors with $98 \%$ system detection efficiency at $1550 \mathrm{~nm}$, Optica, 7, 1649 (2020).

[140] J. Chang, J. W. N. Los, J. O. Tenorio-Pearl, N. Noordzij, R. Gourgues, A. Guardiani, J. R. Zichi, S. F. Pereira, H. P. Urbach, V. Zwiller, S. N. Dorenbos, and I. E. Zadeh, Detecting Infrared Single Photons with Near-Unity System Detection Efficiency, arXiv:2011.08941 (2020).

[141] N. Montaut, L. Sansoni, E. Meyer-Scott, R. Ricken, V. Quiring, H. Herrmann, and C. Silberhorn, High-Efficiency Plug-and-Play Source of Heralded Single Photons, Phys. Rev. Appl., 8, 024021 (2017).

[142] R.-B. Jin, N. Cai, Y. Huang, X.-Y. Hao, S. Wang, F. Li, H.-Z. Song, Q. Zhou, and R. Shimizu, Theoretical Investigation of a Spectrally Pure-State Generation from Isomorphs of KDP Crystal at Near-Infrared and Telecom Wavelengths, Phys. Rev. Appl, 11, 034067 (2019).

[143] F. Laudenbach, R.-B. Jin, C. Greganti, M. Hentschel, P. Walther, and H. Hübel, Numerical investigation of photon-pair generation in periodically poled $m \mathrm{TiO} \mathrm{O}_{4}$ ( $m=\mathrm{K}, r b, c s ; x=p$, as), Phys. Rev. Applied, 8, 024035 (2017).

[144] F. König and F. N. C. Wong, Extended phase matching of second-harmonic generation in periodically poled KTiOPO4 with zero group-velocity mismatch, Appl. Phys. Lett., 84, 1644-1646 (2004).

[145] K. Fradkin, A. Arie, A. Skliar, and G. Rosenman, Tunable midinfrared source by difference frequency generation in bulk periodically poled KTiOPO4, Appl. Phys. Lett., 74, 914-916 (1999).

[146] G. Tamošauskas, G. Beresnevičius, D. Gadonas, and A. Dubietis, Transmittance and phase matching of bbo crystal in the 3-5 $\mu \mathrm{m}$ range and its application for the characterization of mid-infrared laser pulses, Opt. Mater. Express, 8, 1410-1418 (2018).

[147] R. Menzel, Photonics: Linear and Nonlinear Interactions of Laser Light and Matter, Springer Science \& Business Media, 2nd edition edition (2013).

[148] V. Anant, A. J. Kerman, E. A. Dauler, J. K. W. Yang, K. M. Rosfjord, and K. K. Berggren, Optical properties of superconducting nanowire single-photon detectors, Opt. Express, 16, 10750-10761 (2008).

[149] E. F. C. Driessen, F. R. Braakman, E. M. Reiger, S. N. Dorenbos, V. Zwiller, and M. J. A. de Dood, Impedance model for the polarization-dependent optical absorption of superconducting single-photon detectors, EPJ Appl. Phys., 47 (2009). 
[150] C. Toebes, Multi-Photon Source for Integrated Quantum Photonics, Master's thesis, University of Twente, Enschede (2019).

[151] B. Yurke and M. Potasek, Obtainment of thermal noise from a pure quantum state, Phys. Rev. A, 36, 3464-3466 (1987).

[152] K. Kato and E. Takaoka, Sellmeier and thermo-optic dispersion formulas for KTP, Appl. Opt., 41, 5040-5044 (2002).

[153] M. C. Tichy, Sampling of partially distinguishable bosons and the relation to the multidimensional permanent, Phys. Rev. A, 91, 022316 (2015).

[154] F. Somhorst, Quantum Simulation of Thermalization on an Integrated Programmable Photonic Processor, Master's thesis, University of Twente (2021).

[155] W. Bogaerts, D. Pérez, J. Capmany, D. A. B. Miller, J. Poon, D. Englund, F. Morichetti, and A. Melloni, Programmable photonic circuits, Nature, 586, 207216 (2020).

[156] J. Carolan, C. Harrold, C. Sparrow, E. Martín-López, N. J. Russell, J. W. Silverstone, P. J. Shadbolt, N. Matsuda, M. Oguma, M. Itoh, G. D. Marshall, M. G. Thompson, J. C. F. Matthews, T. Hashimoto, J. L. O'Brien, and A. Laing, Universal linear optics, Science, 349, 711-716 (2015).

[157] A. Ribeiro, A. Ruocco, L. Vanacker, and W. Bogaerts, Demonstration of a 4x4-port universal linear circuit, Optica, 3, 1348-1357 (2016).

[158] R. Santagati, J. W. Silverstone, M. J. Strain, M. Sorel, S. Miki, T. Yamashita, M. Fujiwara, M. Sasaki, H. Terai, M. G. Tanner, C. M. Natarajan, R. H. Hadfield, J. L. O'Brien, and M. G. Thompson, Silicon photonic processor of two-qubit entangling quantum logic, J. Opt., 19, 114006 (2017).

[159] N. C. Harris, G. R. Steinbrecher, M. Prabhu, Y. Lahini, J. Mower, D. Bunandar, C. Chen, F. N. C. Wong, T. Baehr-Jones, M. Hochberg, S. Lloyd, and D. Englund, Quantum transport simulations in a programmable nanophotonic processor, Nat. Photonics, 11, 447-452 (2017).

[160] C. Sparrow, E. Martín-López, N. Maraviglia, A. Neville, C. Harrold, J. Carolan, Y. N. Joglekar, T. Hashimoto, N. Matsuda, J. L. O’Brien, D. P. Tew, and A. Laing, Simulating the vibrational quantum dynamics of molecules using photonics, Nature, 557, 660-667 (2018).

[161] X. Qiang, X. Zhou, J. Wang, C. M. Wilkes, T. Loke, S. O’Gara, L. Kling, G. D. Marshall, R. Santagati, T. C. Ralph, J. B. Wang, J. L. O'Brien, M. G. Thompson, and J. C. F. Matthews, Large-scale silicon quantum photonics implementing arbitrary two-qubit processing, Nat. Photonics, 12, 534-539 (2018).

[162] S. Paesani, Y. Ding, R. Santagati, L. Chakhmakhchyan, C. Vigliar, K. Rottwitt, L. K. Oxenløwe, J. Wang, M. G. Thompson, and A. Laing, Generation and sampling of quantum states of light in a silicon chip, Nat. Phys., 15, 925-929 (2019).

[163] C. Taballione, T. A. W. Wolterink, J. Lugani, A. Eckstein, B. A. Bell, R. Grootjans, I. Visscher, D. Geskus, C. G. H. Roeloffzen, J. J. Renema, I. A. Walmsley, P. W. H. Pinkse, and K.-J. Boller, $8 x 8$ reconfigurable quantum photonic processor based on silicon nitride waveguides, Opt. Express, 27, 26842-26857 (2019). 
[164] J. Carolan, M. Mohseni, J. P. Olson, M. Prabhu, C. Chen, D. Bunandar, M. Y. Niu, N. C. Harris, F. N. C. Wong, M. Hochberg, S. Lloyd, and D. Englund, Variational quantum unsampling on a quantum photonic processor, Nat. Phys., 16, 322-327 (2020).

[165] F. Hoch, S. Piacentini, T. Giordani, Z.-N. Tian, M. Iuliano, C. Esposito, A. Camillini, G. Carvacho, F. Ceccarelli, N. Spagnolo, A. Crespi, F. Sciarrino, and R. Osellame, Boson Sampling in a reconfigurable continuously-coupled 3D photonic circuit, arXiv:2106.08260 (2021).

[166] Y. Shen, N. C. Harris, S. Skirlo, M. Prabhu, T. Baehr-Jones, M. Hochberg, X. Sun, S. Zhao, H. Larochelle, D. Englund, and M. Soljačić, Deep learning with coherent nanophotonic circuits, Nat. Photonics, 11, 441-446 (2017).

[167] J. Feldmann, N. Youngblood, M. Karpov, H. Gehring, X. Li, M. Stappers, M. Le Gallo, X. Fu, A. Lukashchuk, A. S. Raja, J. Liu, C. D. Wright, A. Sebastian, T. J. Kippenberg, W. H. P. Pernice, and H. Bhaskaran, Parallel convolutional processing using an integrated photonic tensor core, Nature, 589, 52-58 (2021).

[168] V. Saggio, B. E. Asenbeck, A. Hamann, T. Strömberg, P. Schiansky, V. Dunjko, N. Friis, N. C. Harris, M. Hochberg, D. Englund, S. Wölk, H. J. Briegel, and $\mathrm{P}$. Walther, Experimental quantum speed-up in reinforcement learning agents, Nature, 591, 229-233 (2021).

[169] L. Zhuang, C. G. H. Roeloffzen, M. Hoekman, K.-J. Boller, and A. J. Lowery, Programmable photonic signal processor chip for radiofrequency applications, Optica, 2, 854-859 (2015).

[170] D. Pérez, I. Gasulla, L. Crudgington, D. J. Thomson, A. Z. Khokhar, K. Li, W. Cao, G. Z. Mashanovich, and J. Capmany, Multipurpose silicon photonics signal processor core, Nat. Commun., 8, 636 (2017).

[171] J. M. Arrazola, V. Bergholm, K. Brádler, T. R. Bromley, M. J. Collins, et al., Quantum circuits with many photons on a programmable nanophotonic chip, Nature, 591, 54-60 (2021).

[172] M. Dong, G. Clark, A. J. Leenheer, M. Zimmermann, D. Dominguez, A. J. Menssen, D. Heim, G. Gilbert, D. Englund, and M. Eichenfield, High-speed programmable photonic circuits in a cryogenically compatible, visible-near-infrared $200 \mathrm{~mm}$ CMOS architecture, Nat. Photonics, 16, 59-65 (2022).

[173] J. Carolan, J. D. A. Meinecke, P. J. Shadbolt, N. J. Russell, N. Ismail, K. Wörhoff, T. Rudolph, M. G. Thompson, J. L. O'Brien, J. C. F. Matthews, and A. Laing, On the experimental verification of quantum complexity in linear optics, Nat. Photonics, 8, 621-626 (2014).

[174] J. Wang, S. Paesani, Y. Ding, R. Santagati, P. Skrzypczyk, A. Salavrakos, J. Tura, R. Augusiak, L. Mančinska, D. Bacco, D. Bonneau, J. W. Silverstone, Q. Gong, A. Acín, K. Rottwitt, L. K. Oxenløwe, J. L. O’Brien, A. Laing, and M. G. Thompson, Multidimensional quantum entanglement with large-scale integrated optics, Science, 360, 285-291 (2018). 
[175] T. J. Seok, K. Kwon, J. Henriksson, J. Luo, and M. C. Wu, Wafer-scale silicon photonic switches beyond die size limit, Optica, 6, 490-494 (2019).

[176] C. G. H. Roeloffzen, M. Hoekman, E. J. Klein, L. S. Wevers, R. B. Timens, et al., Low-Loss $\mathrm{Si}_{3} \mathrm{~N}_{4}$ TriPleX Optical Waveguides: Technology and Applications Overview, IEEE J. Sel. Top. Quantum Electron., 24, 1-21 (2018).

[177] W. R. Clements, P. C. Humphreys, B. J. Metcalf, W. S. Kolthammer, and I. A. Walmsley, Optimal design for universal multiport interferometers, Optica, 3, 1460 1465 (2016).

[178] A. Laing and J. L. O'Brien, Super-stable tomography of any linear optical device, arXiv:1208.2868 (2012).

[179] I. Dhand, A. Khalid, H. Lu, and B. C. Sanders, Accurate and precise characterization of linear optical interferometers, J. Opt., 18, 035204 (2016).

[180] P. A. Lee and A. D. Stone, Universal Conductance Fluctuations in Metals, Phys. Rev. Lett., 55, 1622-1625 (1985).

[181] P. G. Murphy and J. E. Moore, Coherent phonon scattering effects on thermal transport in thin semiconductor nanowires, Phys. Rev. B, 76, 155313 (2007).

[182] S. Washburn, Fluctuations in the extrinsic conductivity of disordered metal, IBM J. Res. Dev., 32, 335-346 (1988).

[183] K.-P. Ho and J. M. Kahn, Statistics of Group Delays in Multimode Fiber With Strong Mode Coupling, J. Light. Technol., 29, 3119-3128 (2011).

[184] W. Xiong, C. W. Hsu, Y. Bromberg, J. E. Antonio-Lopez, R. Amezcua Correa, and $\mathrm{H}$. Cao, Complete polarization control in multimode fibers with polarization and mode coupling, Light Sci. Appl., 7, 54 (2018).

[185] R. Sprik, A. Tourin, J. de Rosny, and M. Fink, Eigenvalue distributions of correlated multichannel transfer matrices in strongly scattering systems, Phys. Rev. B, 78, 012202 (2008).

[186] A. Aubry and A. Derode, Random Matrix Theory Applied to Acoustic Backscattering and Imaging In Complex Media, Phys. Rev. Lett., 102, 084301 (2009).

[187] B. Gérardin, J. Laurent, A. Derode, C. Prada, and A. Aubry, Full Transmission and Reflection of Waves Propagating through a Maze of Disorder, Phys. Rev. Lett., 113, 173901 (2014).

[188] J. F. de Boer, M. C. W. van Rossum, M. P. van Albada, T. M. Nieuwenhuizen, and A. Lagendijk, Probability Distribution of Multiple Scattered Light Measured in Total Transmission, Phys. Rev. Lett., 73, 2567-2570 (1994).

[189] S. M. Popoff, G. Lerosey, R. Carminati, M. Fink, A. C. Boccara, and S. Gigan, Measuring the Transmission Matrix in Optics: An Approach to the Study and Control of Light Propagation in Disordered Media, Phys. Rev. Lett., 104, 100601 (2010).

[190] M. Kim, Y. Choi, C. Yoon, W. Choi, J. Kim, Q.-H. Park, and W. Choi, Maximal energy transport through disordered media with the implementation of transmission eigenchannels, Nat. Photonics, 6, 581-585 (2012). 
[191] R. Pappu, B. Recht, J. Taylor, and N. Gershenfeld, Physical One-Way Functions, Science, 297, 2026-2030 (2002).

[192] S. A. Goorden, M. Horstmann, A. P. Mosk, B. Škorić, and P. W. H. Pinkse, Quantum-secure authentication of a physical unclonable key, Optica, 1, 421-424 (2014).

[193] R. Uppu, T. A. W. Wolterink, S. A. Goorden, B. Chen, B. Škorić, A. P. Mosk, and P. W. H. Pinkse, Asymmetric cryptography with physical unclonable keys, Quantum Sci. Technol., 4, 045011 (2019).

[194] S. E. Skipetrov, Information transfer through disordered media by diffuse waves, Phys. Rev. E, 67, 036621 (2003).

[195] I. M. Vellekoop and C. M. Aegerter, Focusing light through living tissue, in Optical Coherence Tomography and Coherence Domain Optical Methods in Biomedicine $X I V$, volume 7554, p. 755430, International Society for Optics and Photonics (2010).

[196] J. Bertolotti, E. G. van Putten, C. Blum, A. Lagendijk, W. L. Vos, and A. P. Mosk, Non-invasive imaging through opaque scattering layers, Nature, 491, 232-234 (2012).

[197] L. V. Amitonova, A. Descloux, J. Petschulat, M. H. Frosz, G. Ahmed, F. Babic, X. Jiang, A. P. Mosk, P. S. J. Russell, and P. W. H. Pinkse, High-resolution wavefront shaping with a photonic crystal fiber for multimode fiber imaging, Opt. Lett., 41, 497-500 (2016).

[198] T. Yeminy and O. Katz, Guidestar-free image-guided wavefront shaping, Sci. Adv., 7, eabf5364 (2021).

[199] B. Redding, S. F. Liew, R. Sarma, and H. Cao, Compact spectrometer based on a disordered photonic chip, Nat. Photonics, 7, 746-751 (2013).

[200] A. Y. Piggott, J. Lu, K. G. Lagoudakis, J. Petykiewicz, T. M. Babinec, and J. Vučković, Inverse design and demonstration of a compact and broadband on-chip wavelength demultiplexer, Nat. Photonics, 9, 374-377 (2015).

[201] T. Liu and A. Fiore, Designing open channels in random scattering media for on-chip spectrometers, Optica, 7, 934-939 (2020).

[202] W. Choi, A. P. Mosk, Q.-H. Park, and W. Choi, Transmission eigenchannels in a disordered medium, Phys. Rev. B, 83, 134207 (2011).

[203] I. M. Vellekoop and A. P. Mosk, Universal Optimal Transmission of Light Through Disordered Materials, Phys. Rev. Lett., 101, 120601 (2008).

[204] Z. Shi and A. Z. Genack, Transmission Eigenvalues and the Bare Conductance in the Crossover to Anderson Localization, Phys. Rev. Lett., 108, 043901 (2012).

[205] V. A. Marčenko and L. A. Pastur, Distribution of eigenvalues for some sets of random matrices, Math. USSR Sb., 1, 457-483 (1967).

[206] M. Oszmaniec, R. Augusiak, C. Gogolin, J. Kołodyński, A. Acín, and M. Lewenstein, Random Bosonic States for Robust Quantum Metrology, Phys. Rev. X, 6, 041044 (2016). 
[207] D. Y. K. Ko and J. C. Inkson, Matrix method for tunneling in heterostructures: Resonant tunneling in multilayer systems, Phys. Rev. B, 38, 9945-9951 (1988).

[208] C. W. J. Beenakker, Random-matrix theory of quantum transport, Rev. Mod. Phys., 69, 731-808 (1997).

[209] M. C. W. van Rossum and T. M. Nieuwenhuizen, Multiple scattering of classical waves: Microscopy, mesoscopy, and diffusion, Rev. Mod. Phys., 71, 313-371 (1999).

[210] Y. Gilead, M. Verbin, and Y. Silberberg, Ensemble-Averaged Quantum Correlations between Path-Entangled Photons Undergoing Anderson Localization, Phys. Rev. Lett., 115, 133602 (2015).

[211] A. Crespi, R. Osellame, R. Ramponi, V. Giovannetti, R. Fazio, L. Sansoni, F. De Nicola, F. Sciarrino, and P. Mataloni, Anderson localization of entangled photons in an integrated quantum walk, Nat. Photonics, 7, 322-328 (2013).

[212] S. Stützer, Y. Plotnik, Y. Lumer, P. Titum, N. H. Lindner, M. Segev, M. C. Rechtsman, and A. Szameit, Photonic topological Anderson insulators, Nature, 560, 461-465 (2018).

[213] N. Bender, A. Yamilov, H. Y1lmaz, and H. Cao, Fluctuations and Correlations of Transmission Eigenchannels in Diffusive Media, Phys. Rev. Lett., 125, 165901 (2020).

[214] R. Sarma, A. G. Yamilov, S. Petrenko, Y. Bromberg, and H. Cao, Control of Energy Density inside a Disordered Medium by Coupling to Open or Closed Channels, Phys. Rev. Lett., 117, 086803 (2016).

[215] A. Deshpande, A. Mehta, T. Vincent, N. Quesada, M. Hinsche, M. Ioannou, L. Madsen, J. Lavoie, H. Qi, J. Eisert, D. Hangleiter, B. Fefferman, and I. Dhand, Quantum Computational Supremacy via High-Dimensional Gaussian Boson Sampling, arXiv:2102.12474 (2021).

[216] S. Aaronson and D. J. Brod, BosonSampling with lost photons, Phys. Rev. A, 93, 012335 (2016).

[217] E. Knill, R. Laflamme, and G. J. Milburn, A scheme for efficient quantum computation with linear optics, Nature, 409, 46-52 (2001).

[218] A. Crespi, Suppression laws for multiparticle interference in Sylvester interferometers, Phys. Rev. A, 91, 013811 (2015).

[219] M. Bentivegna, N. Spagnolo, and F. Sciarrino, Is my boson sampler working?, New J. Phys., 18, 041001 (2016).

[220] C. Dittel, R. Keil, and G. Weihs, Many-body quantum interference on hypercubes, Quantum Sci. Technol., 2, 015003 (2017).

[221] N. Viggianiello, F. Flamini, L. Innocenti, D. Cozzolino, M. Bentivegna, N. Spagnolo, A. Crespi, D. J. Brod, E. F. Galvão, R. Osellame, and F. Sciarrino, Experimental generalized quantum suppression law in Sylvester interferometers, New J. Phys., 20, 033017 (2018).

[222] T. Giordani, F. Flamini, M. Pompili, N. Viggianiello, N. Spagnolo, A. Crespi, 
R. Osellame, N. Wiebe, M. Walschaers, A. Buchleitner, and F. Sciarrino, Experimental statistical signature of many-body quantum interference, Nat. Photonics, 12, 173-178 (2018).

[223] N. Viggianiello, F. Flamini, M. Bentivegna, N. Spagnolo, A. Crespi, D. J. Brod, E. F. Galvão, R. Osellame, and F. Sciarrino, Optimal photonic indistinguishability tests in multimode networks, Sci. Bull., 63, 1470-1478 (2018).

[224] I. Agresti, N. Viggianiello, F. Flamini, N. Spagnolo, A. Crespi, R. Osellame, N. Wiebe, and F. Sciarrino, Pattern Recognition Techniques for Boson Sampling Validation, Phys. Rev. X, 9, 011013 (2019).

[225] B. M. Terhal, Bell inequalities and the separability criterion, Phys. Lett. A, 271, 319-326 (2000).

[226] V. S. Shchesnovich, Universality of Generalized Bunching and Efficient Assessment of Boson Sampling, Phys. Rev. Lett., 116, 123601 (2016).

[227] I. Gerhardt, Q. Liu, A. Lamas-Linares, J. Skaar, V. Scarani, V. Makarov, and C. Kurtsiefer, Experimentally faking the violation of bell's inequalities, Phys. Rev. Lett., 107, 170404 (2011).

[228] S. Pironio, A. Acín, S. Massar, A. B. de La Giroday, D. N. Matsukevich, P. Maunz, S. Olmschenk, D. Hayes, L. Luo, T. A. Manning, et al., Random numbers certified by bell's theorem, Nature, 464, 1021 (2010).

[229] A. Acín and L. Masanes, Certified randomness in quantum physics, Nature, 540, 213-219 (2016).

[230] N. Brunner, D. Cavalcanti, S. Pironio, V. Scarani, and S. Wehner, Bell nonlocality, Rev. Mod. Phys., 86, 419 (2014).

[231] W.-Z. Liu, M.-H. Li, S. Ragy, S.-R. Zhao, B. Bai, Y. Liu, P. J. Brown, J. Zhang, R. Colbeck, J. Fan, Q. Zhang, and J.-W. Pan, Device-independent randomness expansion against quantum side information, Nat. Phys., 17, 448-451 (2021).

[232] L. K. Shalm, Y. Zhang, J. C. Bienfang, C. Schlager, M. J. Stevens, M. D. Mazurek, C. Abellan, W. Amaya, M. W. Mitchell, M. A. Alhejji, H. Fu, J. Ornstein, R. P. Mirin, S. W. Nam, and E. Knill, Device-independent randomness expansion with entangled photons, Nat. Phys., 17, 452-456 (2021).

[233] M. Pawłowski and N. Brunner, Semi-device-independent security of one-way quantum key distribution, Phys. Rev. A, 84, 010302 (2011).

[234] Z. Cao, H. Zhou, X. Yuan, and X. Ma, Source-independent quantum random number generation, Phys. Rev. X, 6, 011020 (2016).

[235] D. G. Marangon, G. Vallone, and P. Villoresi, Source-device-independent ultrafast quantum random number generation, Phys. Rev. Lett., 118, 060503 (2017).

[236] D. Drahi, N. Walk, M. J. Hoban, A. K. Fedorov, R. Shakhovoy, A. Feimov, Y. Kurochkin, W. S. Kolthammer, J. Nunn, J. Barrett, and I. A. Walmsley, Certified Quantum Random Numbers from Untrusted Light, Phys. Rev. X, 10, 041048 (2020). 
[237] A. Chaturvedi and M. Banik, Measurement-device-independent randomness from local entangled states, EPL, 112, 30003 (2015).

[238] Z. Cao, H. Zhou, and X. Ma, Loss-tolerant measurement-device-independent quantum random number generation, New J. Phys., 17, 125011 (2015).

[239] T. V. Himbeeck, E. Woodhead, N. J. Cerf, R. García-Patrón, and S. Pironio, Semidevice-independent framework based on natural physical assumptions, Quantum, 1, 33 (2017).

[240] J. B. Brask, A. Martin, W. Esposito, R. Houlmann, J. Bowles, H. Zbinden, and N. Brunner, Megahertz-rate semi-device-independent quantum random number generators based on unambiguous state discrimination, Phys. Rev. Appl., 7, 054018 (2017).

[241] A. Máttar, P. Skrzypczyk, G. Aguilar, R. Nery, P. S. Ribeiro, S. Walborn, and D. Cavalcanti, Experimental multipartite entanglement and randomness certification of the $w$ state in the quantum steering scenario, Quantum Sci. Technol., 2, 015011 (2017).

[242] S. Kocsis, M. J. W. Hall, A. J. Bennet, D. J. Saunders, and G. J. Pryde, Experimental measurement-device-independent verification of quantum steering, Nat. Commun., 6, 5886- (2015).

[243] H. D. Ursell, The evaluation of gibbs' phase-integral for imperfect gases, Math. Proc. Cambridge Philos. Soc., 23, 685-697 (1927).

[244] M. Walschaers, Signatures of many-particle interference, J. Phys. B: At. Mol. Opt. Phys., 53, 043001 (2020).

[245] P. G. Evans, R. S. Bennink, W. P. Grice, T. S. Humble, and J. Schaake, Bright Source of Spectrally Uncorrelated Polarization-Entangled Photons with Nearly Single-Mode Emission, Phys. Rev. Lett., 105, 253601 (2010).

[246] F. Marsili, F. Najafi, E. Dauler, F. Bellei, X. Hu, M. Csete, R. J. Molnar, and K. K. Berggren, Single-Photon Detectors Based on Ultranarrow Superconducting Nanowires, Nano Lett., 11, 2048-2053 (2011).

[247] I. Holzman and Y. Ivry, Superconducting Nanowires for Single-Photon Detection: Progress, Challenges, and Opportunities, Adv. Quantum Technol., 2, 1800058 (2019).

[248] A. Feito, J. S. Lundeen, H. Coldenstrodt-Ronge, J. Eisert, M. B. Plenio, and I. A. Walmsley, Measuring measurement: Theory and practice, New J. Phys., 11, 093038 (2009).

[249] J. v. Neumann, Beweis des Ergodensatzes und des H-Theorems in der neuen Mechanik, Z. Phys., 57, 30 (1929).

[250] J. Eisert, M. Friesdorf, and C. Gogolin, Quantum many-body systems out of equilibrium, Nat. Phys., 11, 124-130 (2015).

[251] A. Polkovnikov, K. Sengupta, A. Silva, and M. Vengalattore, Nonequilibrium dynamics of closed interacting quantum systems, Rev. Mod. Phys., 83, 863-883 (2011). 
[252] C. Gogolin and J. Eisert, Equilibration, thermalisation, and the emergence of statistical mechanics in closed quantum systems, Rep. Prog. Phys., 79, 56001 (2016).

[253] M. Cramer, C. M. Dawson, J. Eisert, and T. J. Osborne, Exact relaxation in a class of non-equilibrium quantum lattice systems, Phys. Rev. Lett., 100, 030602 (2008).

[254] M. Cramer and J. Eisert, A quantum central limit theorem for non-equilibrium systems: Exact local relaxation of correlated states, New J. Phys., 12, 055020 (2010).

[255] M. Gluza, J. Eisert, and T. Farrelly, Equilibration towards generalized gibbs ensembles in non-interacting theories, SciPost Phys., 7, 038 (2019).

[256] M. Rigol, V. Dunjko, and M. Olshanii, Thermalization and its mechanism for generic isolated quantum systems, Nature, 452, 854-858 (2008).

[257] A. C. Cassidy, C. W. Clark, and M. Rigol, Generalized thermalization in an integrable lattice system, Phys. Rev. Lett., 106, 140405 (2011).

[258] B. Wouters, M. Brockmann, J. De Nardis, D. Fioretto, M. Rigol, and J.-S. Caux, Quenching the anisotropic Heisenberg chain: Exact solution and generalized Gibbs ensemble, Phys. Rev. Lett., 113, 117202 (2014).

[259] P. Calabrese, F. H. L. Essler, and M. Fagotti, Quantum quench in the transverse-field ising chain, Phys. Rev. Lett., 106, 227203 (2011).

[260] T. Langen, S. Erne, R. Geiger, B. Rauer, T. Schweigler, M. Kuhnert, W. Rohringer, I. E. Mazets, T. Gasenzer, and J. e. a. Schmiedmayer, Experimental observation of a generalized gibbs ensemble, Science, 348, 207-211 (2015).

[261] C. Neill, P. Roushan, M. Fang, Y. Chen, M. Kolodrubetz, Z. Chen, A. Megrant, R. Barends, B. Campbell, and B. e. a. Chiaro, Ergodic dynamics and thermalization in an isolated quantum system, Nat. Phys., 12, 1037-1041 (2016).

[262] S. Trotzky, Y.-A. Chen, A. Flesch, I. P. McCulloch, U. Schollwöck, J. Eisert, and I. Bloch, Probing the relaxation towards equilibrium in an isolated strongly correlated one-dimensional Bose gas, Nat. Phys., 8, 325-330 (2012).

[263] M. Gring, M. Kuhnert, T. Langen, T. Kitagawa, B. Rauer, M. Schreitl, I. Mazets, D. A. Smith, E. Demler, and J. e. a. Schmiedmayer, Relaxation and prethermalization in an isolated quantum system, Science, 337, 1318-1322 (2012).

[264] J. Smith, A. Lee, P. Richerme, B. Neyenhuis, P. W. Hess, P. Hauke, M. Heyl, D. A. Huse, and C. Monroe, Many-body localization in a quantum simulator with programmable random disorder, Nat. Phys., 12, 907-911 (2016).

[265] G. Clos, D. Porras, U. Warring, and T. Schaetz, Time-resolved observation of thermalization in an isolated quantum system, Phys. Rev. Lett., 117 (2016).

[266] R. Islam, R. Ma, P. M. Preiss, M. E. Tai, A. Lukin, M. Rispoli, and M. Greiner, Measuring entanglement entropy in a quantum many-body system, Nature, 528, 77-83 (2015).

[267] A. Aspuru-Guzik and P. Walther, Photonic quantum simulators, Nat. Phys., 8, 285-291 (2012). 
[268] M. C. Rechtsman, J. M. Zeuner, Y. Plotnik, Y. Lumer, D. Podolsky, F. Dreisow, S. Nolte, M. Segev, and A. Szameit, Photonic floquet topological insulators, Nature, 496, 196-200 (2013).

[269] M. Lubasch, A. A. Valido, J. J. Renema, W. S. Kolthammer, D. Jaksch, M. S. Kim, I. Walmsley, and R. García-Patrón, Tensor network states in time-bin quantum optics, Phys. Rev. A, 97, 062304 (2018).

[270] I. Dhand, M. Engelkemeier, L. Sansoni, S. Barkhofen, C. Silberhorn, and M. B. Plenio, Proposal for quantum simulation via all-optically-generated tensor network states, Phys. Rev. Lett., 120, 130501 (2018).

[271] J. Eisert, D. Hangleiter, N. Walk, I. Roth, D. Markham, R. Parekh, U. Chabaud, and E. Kashefi, Quantum certification and benchmarking, Nat Rev Phys, 2, 382-390 (2020).

[272] L. Aolita, C. Gogolin, M. Kliesch, and J. Eisert, Reliable quantum certification for photonic quantum technologies, Nat. Commun., 6, 8498 (2015).

[273] S. Rahimi-Keshari, M. A. Broome, R. Fickler, A. Fedrizzi, T. C. Ralph, and A. G. White, Direct characterization of linear-optical networks, Opt. Express, 21, 13450 (2013).

[274] M. C. Tichy, M. Tiersch, F. de Melo, F. Mintert, and A. Buchleitner, ZeroTransmission Law for Multiport Beam Splitters, Phys. Rev. Lett., 104, 220405 (2010).

[275] M. C. Tichy, M. Tiersch, F. Mintert, and A. Buchleitner, Many-particle interference beyond many-boson and many-fermion statistics, New J. Phys., 14, 093015 (2012).

[276] M. C. Tichy, K. Mayer, A. Buchleitner, and K. Mølmer, Stringent and Efficient Assessment of Boson-Sampling Devices, Phys. Rev. Lett., 113, 020502 (2014).

[277] N. Friis, G. Vitagliano, M. Malik, and M. Huber, Entanglement certification from theory to experiment, Nat. Rev. Phys., 1, 72-87 (2019).

[278] N. C. Harris, J. Carolan, D. Bunandar, M. Prabhu, M. Hochberg, T. Baehr-Jones, M. L. Fanto, A. M. Smith, C. C. Tison, P. M. Alsing, and D. Englund, Linear programmable nanophotonic processors, Optica, 5, 1623-1631 (2018).

[279] F. Mezzadri, How to generate random matrices from the classical compact groups, ArXiv:0609050 (2006).

[280] R. van der Meer, J. J. Renema, B. Brecht, C. Silberhorn, and P. W. H. Pinkse, Optimizing spontaneous parametric down-conversion sources for boson sampling, Phys. Rev. A, 101, 063821 (2020).

[281] F. Samara, A. Martin, C. Autebert, M. Karpov, T. J. Kippenberg, H. Zbinden, and $\mathrm{R}$. Thew, High-rate photon pairs and sequential Time-Bin entanglement with $\mathrm{Si}_{3} \mathrm{~N}_{4}$ microring resonators, Opt. Express, 27, 19309-19318 (2019).

[282] Y. Zhao, Y. Okawachi, J. K. Jang, X. Ji, M. Lipson, and A. L. Gaeta, NearDegenerate Quadrature-Squeezed Vacuum Generation on a Silicon-Nitride Chip, Phys. Rev. Lett., 124, 193601 (2020).

[283] Y. Zhang, M. Menotti, K. Tan, V. D. Vaidya, D. H. Mahler, L. G. Helt, L. Zatti, 
M. Liscidini, B. Morrison, and Z. Vernon, Squeezed light from a nanophotonic molecule, Nat. Commun., 12, 2233 (2021).

[284] P. Lodahl, Quantum-dot based photonic quantum networks, Quantum Sci. Technol., 3, 013001 (2017).

[285] F. Lenzini, B. Haylock, J. C. Loredo, R. A. Abrahão, N. A. Zakaria, S. Kasture, I. Sagnes, A. Lemaitre, H.-P. Phan, D. V. Dao, P. Senellart, M. P. Almeida, A. G. White, and M. Lobino, Active demultiplexing of single photons from a solid-state source, Laser Photonics Rev., 11, 1600297 (2017).

[286] H. Ollivier, I. Maillette de Buy Wenniger, S. Thomas, S. C. Wein, A. Harouri, G. Coppola, P. Hilaire, C. Millet, A. Lemaître, I. Sagnes, O. Krebs, L. Lanco, J. C. Loredo, C. Antón, N. Somaschi, and P. Senellart, Reproducibility of HighPerformance Quantum Dot Single-Photon Sources, ACS Photonics, 7, 1050-1059 (2020).

[287] N. Hosseini, R. Dekker, M. Hoekman, M. Dekkers, J. Bos, A. Leinse, and R. Heideman, Stress-optic modulator in TriPleX platform using a piezoelectric lead zirconate titanate (PZT) thin film, Opt. Express, 23, 14018-14026 (2015).

[288] J. P. Epping, D. Marchenko, A. Leinse, R. Mateman, M. Hoekman, L. Wevers, E. J. Klein, C. G. H. Roeloffzen, M. Dekkers, and R. G. Heideman, Ultra-lowpower stress-optics modulator for microwave photonics, in S. M. García-Blanco and G. N. Conti (editors), Integrated Optics: Devices, Materials, and Technologies XXI, volume 10106, pp. 80 - 87, International Society for Optics and Photonics, SPIE (2017).

[289] P. R. Stanfield, A. J. Leenheer, C. P. Michael, R. Sims, and M. Eichenfield, CMOScompatible, piezo-optomechanically tunable photonics for visible wavelengths and cryogenic temperatures, Opt. Express, 27, 28588-28605 (2019).

[290] N. Quack, H. Sattari, A. Y. Takabayashi, Y. Zhang, P. Verheyen, W. Bogaerts, P. Edinger, C. Errando-Herranz, and K. B. Gylfason, MEMS-Enabled Silicon Photonic Integrated Devices and Circuits, IEEE Journal of Quantum Electronics, 56, 1-10 (2020).

[291] X. Hu, C. W. Holzwarth, D. Masciarelli, E. A. Dauler, and K. K. Berggren, Efficiently Coupling Light to Superconducting Nanowire Single-Photon Detectors, IEEE Trans. Appl. Supercond., 19, 336-340 (2009).

[292] C. Schuck, W. H. P. Pernice, and H. X. Tang, Waveguide integrated low noise NbTiN nanowire single-photon detectors with milli-Hz dark count rate, Sci. Rep., 3, 1893 (2013).

[293] P. W. Anderson, Absence of Diffusion in Certain Random Lattices, Phys. Rev., 109, 1492-1505 (1958).

[294] J.-H. Kim, S. Aghaeimeibodi, J. Carolan, D. Englund, and E. Waks, Hybrid integration methods for on-chip quantum photonics, Optica, 7, 291-308 (2020). 


\section{Nederlandse samenvatting}

Aan het begin van dit promotiewerk, begin 2018, werd de mogelijkheid om een zogenaamde ' quantum advantage' volop bediscussieerd in de wetenschappelijke gemeenschap. Echter, het maximaal aantal identieke fotonen in elk experiment was nog altijd op een hand te tellen en het aantal optische kanalen in een optisch netwerk was amper groter. Daarbovenop waren de verliezen te hoog voor een schaalbaar systeem. Deze situatie veranderde snel in de afgelopen jaren, hetgeen uiteindelijk leidde tot een geclaimde observatie van een quantum advantage, eerst met supergeleidende qubits en vlak daarna met fotonen. Deze ontwikkelingen veroorzaakten een opleving van nieuwe ontwikkelingen in nieuwe en grotere optische netwerken met lage verliezen, en betere enkel-foton-bronnen en detectoren. Dit proefschrift situeert zich in deze context. Het proefschrift biedt nieuwe inzichten in enkel-foton-bronnen, optische netwerken en geeft een demonstratie van eerste toepassingen van dergelijke technologie.

Het proefschrift begint met de introductie van een nieuw optisch netwerk dat op een natuurlijke wijze toegang verschaft tot vele optische modes en weinig verliezen. Dit netwerk kan gerealiseerd worden met behulp van een oppervlakte verstrooier of faseplaat. Alhoewel het netwerk niet instelbaar is, is het netwerk nog steeds geschikt voor een quantum advantage experiment. Boson sampling gaat namelijk om het zoveel mogelijk uitkleden van een quantumcomputer, onder voorbehoud dat de computationele complexiteit van het experiment maar behouden blijft. Om die reden wordt de complexiteit van het netwerk zowel theoretisch alsmede numeriek bestudeerd. Gegeven deze computationele complexiteit, dit netwerk is geschikt voor een optische quantum-advantage-experiment met significant meer modes en een sterke reductie van verliezen.

Het volgende missende puzzelstuk voor een multifoton-experiment is een multifotonbron die tegelijkertijd meerdere identieke enkele fotonen produceert. Traditioneel wordt hiervoor sponteanous parametric downconversion, ook wel afgekort tot SPDC, gebruikt. Dit type van enkelfoton-bronnen gebruikt een niet-lineair optisch kristal die probabilistisch enkelfoton-paren genereert. De geschiktheid van SPDC-bronnen voor multifotonexperimenten was lange tijd een open probleem in verband met de verliezen en probabilistische generatie. We laten zien dat de optimale SPDC-bron bestaat uit een aperiodieke gepoolde KTP-kristal die collineair fotonparen produceert op $1582 \mathrm{~nm}$. Deze bron kan opgeschaald worden tot een maximum van 11500 identieke fotonen die nog steeds quantum mechanisch kunnen interfereren. De inzichten van deze studie zijn tevens gebruikt om onze $1550 \mathrm{~nm}$ SPDC-bron te ontwerpen. Onze bron bestaat uit twee KTP kristallen met een periodiek pooling. Elk kristal genereert $0.2 \mathrm{MHz}$ fotonparen, met een heralding efficiëntie van 0.45 en een foton-ononderscheidbaarheid van 0.89 zonder spectrale filtering. Deze bron is goed genoeg om op te schalen naar een totaal van 11 identieke fotonen.

Het laatste puzzelstuk voor multifoton-experimenten is een grootschalig optisch netwerk waar de fotonen volop gebruik van kunnen maken. Een veelbelovende manier om een dergelijk groot en instelbaar netwerk te realiseren met weinig verliezen is geïntegreerde fotonica. We introduceren een state-of-the-art siliciumnitride quantum-processor. De processor heeft 12 input en 12 output modes waartussen de fotonen op een arbitraire manier kunnen worden gestuurd ten koste van gemiddeld $2.2 \mathrm{~dB}$ verlies. De implementatie 
van willekeurig gekozen transfer matrices gaat met een gemiddelde amplitude fidelity van 0.99. De fidelity met enkele fotonen is iets lager, rond de 0.90 . Deze processor is, samen met de multifoton-bron, de centrale bouwsteen voor alle experimenten is dit proefschrift.

Het eerst uitgevoerde experiment op de quantum-processor is een experimentele demonstratie van de bimodale verdeling en bijbehorende open kanalen in mesoscopische media. Open kanalen zijn de tegen-intuïtieve voorspelling uit de jaren 80 dat golfpropagatie door een ongeordend systeem beschreven kan worden door kanalen die ofwel volledig reflecteren, dan wel volledige transmissie vertonen. Deze kanalen worden ook wel respectievelijk gesloten en open kanalen genoemd. Ondanks vele experimentele pogingen, is deze bimodale verdeling van transmissiekanalen nog nooit overtuigend experimenteel waargenomen. Wij laten met behulp van quantuminterferentie experimenteel bewijs zien dat deze bimodale verdeling daadwerkelijk bestaat. Dit experimentele resultaat kan uitsluitend gemeten worden als aan alle kanalen van het netwerk gemeten kan worden. De quantum-processor is een zeldzaam voorbeeld van een optisch systeem waar dit voor geldt. Dit resultaat onderstreept de geschiktheid van de processor voor fysische simulaties.

$\mathrm{Nu}$ de geschiktheid van de processor voor multifoton-interferentie aangetoond is, kan men zich afvragen hoe multifoton-interferentie gecertificeerd kan worden in een groot arbitrair netwerk. Immers, de quantuminterferentie van enkele fotonen is een voorbeeld van een fysisch proces dat een klassieke computer niet efficiënt kan uitvoeren. Als antwoord op dit probleem introduceren wij een one-sided device-independent photonic indistinguishability witness. Deze witness is gebaseerd op een numeriek efficiënte twee-mode correlator die eenvoudig gemeten kan worden met photon-number-resolving detectoren. De witness is one-sided device independent omdat elke experimentele imperfectie in de processor of detectoren onherroepelijk resulteert in een reductie van de waargenomen correlator. Dit betekent dat elke gemeten correlator dient als een ondergrens voor multifoton-interferentie en dat de methode robuust is tegen experimentele imperfecties. We tonen de bruikbaarheid van deze witness aan met een drie-foton-experiment.

Tot slot onderzoekt dit proefschrift de vraag hoe een pure quantumtoestand thermisch gedrag kan vertonen in een gesloten systeem. Hiervoor simuleren we de tijdsevolutie van zowel een niet interacterende Bose-Hubbard superfluïde, alsmede verschillende willekeurige long-range Hamiltonianen op de quantum-processor. We laten zien dat met de tijd, alhoewel de gehele quantum toestand in deze systemen puur is, het alsnog lokaal een thermische (gemengde) toestand benadert. De lokaal thermische toestand volgt van de toestands verstrengeling met de fotonen in het 'universum' -de niet geobserveerde kanalen van het systeem. Deze hypothese wordt gestaafd door een certificatieprotocol waarbij de reversibiliteit van het systeem gebruikt wordt om de verstrengeling ongedaan te maken en aan te tonen dat de fotonen nog steeds niet onderscheidbaar zijn.

In het kort, dit proefschrift beschrijft een state-of-the-art multifoton-bron die gebruikt is voor de eerste quantum experimenten op een veelbelovend nieuw platform: de geïntegreerde optische quantum-processor. Deze experimenten lossen een vier-decennia-oud probleem op, introduceren een nieuwe robuuste indistinguishability witness en tot slot bewijzen dat verstrengeling zorgt dat pure quantum toestanden zich lokaal thermisch gedragen. 


\section{Acknowledgements}

Any achievement, being academic or personal, is never only the result of the person's own efforts, much rather it is the result of interactions with many different people that help shape ideas, knowledge and the very path towards it. This thesis is the culmination of years of work during my PhD. Luckily, I have been fortunate to have many people around to support and help me during my $\mathrm{PhD}$ with various things such as theory, experiments, discussions and support in general. This thesis could not have been written without the help and support of any of these people.

My gratitude goes out to my promotor Pepijn Pinkse. Your enthusiasm for science is contagious and your kindness and support during the inevitable moments I hit a dead end contributed to the wide variety of successful experiments. Not only was your door always open for discussions, but you also insisted on always hearing the latest (good) results from the lab immediately. And whenever something would not work, you would immediately make time to discuss ways to solve the problems. Furthermore, you would come up with all sorts of out-of-the-box and fun suggestions and ideas. Moreover, in the last years you set up the new Adaptive Quantum Optics (AQO) group, which has proven to be a safe, productive and pleasant work environment. This turned out to be essential for doing many experiments in a short amount of time.

Secondly, I want to thank my co-promotor Jelmer Renema. Initially, we started out working together in the lab, for example, to realign Ti:Sapphire lasers. Soon after, you pushed me down the rabbit role of linear algebra and boson sampling theory. From Jelmer I learned a lot about not just these topics, but also about a large amount of random trivia such as about linguistics, history, alpacas and birds. I appreciated both your insights, your ideas and the positive energy you create.

I would like to thank Jan klärs for his contributions to the AQO group.

There are many external collaborators that contributed to the content of this thesis. Benni Brecht and Christine Silberhorn from Paderborn University for their help in the theoretical investigation of the suitability of SPDC sources. Raul Garcia-Patron from the University of Edinburgh for his insights in the theory of boson sampling. Jens Eisert, Nathan Walk and Riko Schadow from the Free University of Berlin for all (pedantic) contributions to quantum many-body theory and certification theory. Allard Mosk from Utrecht University for his insights in scattering and random matrix theory and proofreading. Mehul Malik from Heriot-Watt University for leading the European consortium. I thank both Hans Hilgenkamp and Klaus Boller for proofreading drafts of various papers.

I would also like to thank all people involved in the loop quantumgravity project: Zichang Huang, Hongguang Liu, Dongxue Qu, Muxin Han and Lior Cohen. Unfortunately, this project was only completed shortly after the deadline of this thesis so it is not included in here. I am sure it will result in a nice paper.

There were quite a few students who I either supervised, or collaborated with on various projects: Chris, Frank, Maarten, Peter H and Pim. To Chris and Pim I am indebted to for building the first version of the $1550 \mathrm{~nm}$ source photon source. I greatly enjoyed working again with my good friend Peter on the visibility witness and loop quantum gravity projects. You did a great job and should be proud of how you managed to complete these projects. 
Frank, you did a good job with both setting up the detector (electronics) and the whole thermalization project. I enjoyed working with all of you and would like to thank you all for your contributions. Your great work allowed to progress many projects forward and culminated in nice papers.

Regarding experimental work, I want to thank Malaquias as well. You started about a year ago and are a theorist by training. Nonetheless, you managed to catch up quickly and help me out a lot during the many hours in the lab.

All experimental work with the quantum processor have only been made possible by the people of Quix: Ben Kassenberg, Caterina Taballione, Devin Smith, Hans van den Vlekkert, Henk Snijders, Jörn Epping, Michiel de Goede, Pim Venderbosch. Your help and troubleshooting with both software and hardware problems we encountered were not just quix, but also essential to the success of all experiments.

Besides the standard work during this $\mathrm{PhD}$ project, I also co-organized the monthly applied nanophotonics (ANP) meetings with both Ivo Hegeman and Yvan Klaver. I would like to thank you both for the effort and help in making these sessions possible. For support with technical and administrative tasks at the university I would like to thank: Bert, Cock, Marlon, Marnix, Melissa and Nicole.

One of the advantages of doing a $\mathrm{PhD}$ is that you spend a lot of time with people of similar age. This naturally encourages close contact and much-needed relaxation outside of the hard (lab)work. The many nice -and sometimes utterly bizarre- coffee and lunch-break discussions, Friday evening beers -also the digital ones during the lockdown- and all other sorts of casual occasions were absolutely great. Many thanks to the people of the AQO group: Charlie, Chris, Daan, Evangelos, Frank, Innes, Jardi, Kayleigh, Klaasjan, LarsvdH, Lucas, Maarten, Malaquias, Mario, Marius, Matthijs, Peter H, Pim, Ravi, Shivan, Stefan, Sudhir, Tom, Tristan, Violetta and Willemijn. From COPS: Alfredo, Andreas, Devasish, Diana, Duy, Femi, Lars, Manashee, Marek, Maryna, Ozan, Shakeeb, Shravan, Shweta, Sjoerd and Timon. Finally from LPNO: Albert, Anzal, Bert, Carla, Caterina, Edith, Haider, Jasper, Jesse, Kaixuan, Kasper, Kees, Lisa, Lisi, Marco, Max, Okky, Peter vdS, Radius, Redlef, Roel, Xin, Yin, Youwen and Yvan.

I indebted to my family. My parents, who raised and supported me throughout my life. Your support forms the solid bedrock of my existence, on which I could develop and build my own life. My brother Thomas, who is similar but yet so different from me. It is good to have a fellow physicist in the family too.

Finally, I want to thank Manashee. In the last few years, you have supported me throughout and kept me sane during the Corona pandemic. I admire your strength, persistence, and intelligence you showed in not just exploring, but also thriving in uncharted places. Furthermore, despite being limited in traveling anywhere: you managed to open a whole new world and culture to me. I look forward to see where the future will take us. 


\section{List of publications}

\section{Journal articles}

1. T. Dennis, M.W. van Dijk, J.H.H. Lee, R. van der Meer, A. Strooisma, P.J.M. van der Slot, W.L. Vos, and K.-J. Boller, "Coherent Cherenkov radiation and laser oscillation in a photonic crystal", Phys. Rev. A. 94053852 (2016)

2. T.B.H. Tentrup, W.M. Luiten, R. van der Meer, P. Hooijschuur, and P.W.H. Pinkse, Large-alphabet quantum key distribution using spatially encoded light, New J. Phys. 21123044 (2019)

3. R. van der Meer, J.J. Renema, B. Brecht, C. Silberhorn, and P.W.H. Pinkse, "Optimizing spontaneous parametric down-conversion sources for boson sampling", Phys. Rev. A. 101063821 (2020)

4. C. Taballione, R. van der Meer, H.J Snijders, P. Hooijschuur, J.P. Epping, M. de Goede, B. Kassenberg, P. Venderbosch, C. Toebes, H. van den Vlekkert, P.W.H. Pinkse, and J.J. Renema, "A universal fully reconfigurable 12-mode quantum photonic processor", Mater. Quantum Technol. 1035002 (2021)

5. R. van der Meer, S.Huber, P.W.H. Pinkse, and J.J. Renema, "Boson Sampling in Low-depth Optical Systems", (submitted)

6. R. van der Meer, M. de Goede, B. Kassenberg, P. Venderbosch, H. Snijders, J.P. Epping, C. Taballione , H. van den Vlekkert, J.J. Renema, and P.W.H. Pinkse, "Observation of open scattering channels", (submitted)

7. R. van der Meer, P. Hooijschuur, F.H.B. Somhorst, P. Venderbosch, M.de Goede, B. Kassenberg, H.J. Snijders, C. Taballione, J. Epping, H. van den Vlekkert, N. Walk, P.W.H. Pinkse, and J.J. Renema, "One-sided device-independent indistinguishability witness", (submitted)

8. F.H.B. Somhorst, R. van der Meer, M. Correa Anguita, R. Schadow, H.J. Snijders, M. de Goede, B. Kassenberg, P. Venderbosch, C. Taballione, J. Epping, H. Vlekkert, J.F.F. Bulmer, J. Lugani, I.A. Walmsley, P.W.H. Pinkse, J. Eisert, N. Walk, and J.J. Renema, "Quantum photo-thermodynamics on a programmable photonic quantum processor", (submitted)

9. "Experimental Simulation of Loop Quantum Gravity on a Photonic Chip" (in preparation)

10. "A 20-mode quantum photonic processor" (in preparation)

\section{Conference Proceedings}

1. T.B.H. Tentrup, W.M. Luiten, P. Hooijschuur, R. van der Meer, and P.W.H. Pinkse, "Two high-dimensional cartesian bases for quantum key distribution", $6^{\text {th }}$ Workshop on Information Optics (2017) 
2. J.P. Epping, C. Taballione, R. van der Meer, H.J. Snijders, P. Hooijschuur, B. Kassenberg, M. de Goede, P. Venderbosch, c. Toebes, H. van den Vlekkert, P.W.H. Pinkse, and J.J. Renema, "Programmable quantum photonic processor based on intetrated silicon nitride waveguides", Quantum Computing, Communication, and Simulation (2021)

3. R. van der Meer, J.P. Epping, C. Taballione, H. Snijders, P. Hooischuur, B. Kassenberg, M. de Goede, P. Venderbosch, C. Toebes, H. van den Vlekkert, P.W.H. Pinkse, J.J. Renema, "High-Fidelity Integrated Silicon Nitride Quantum Photonic Processor" (in preparation) 